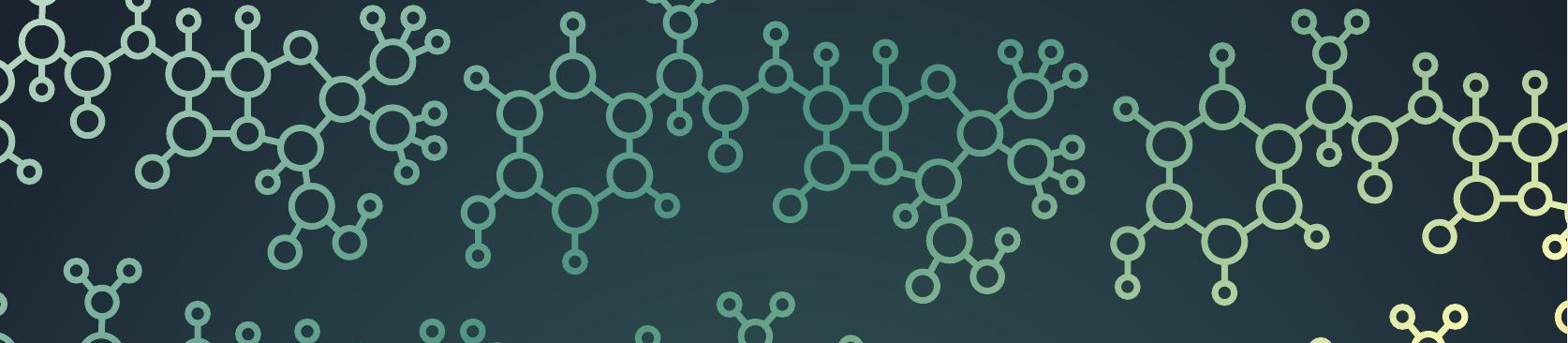

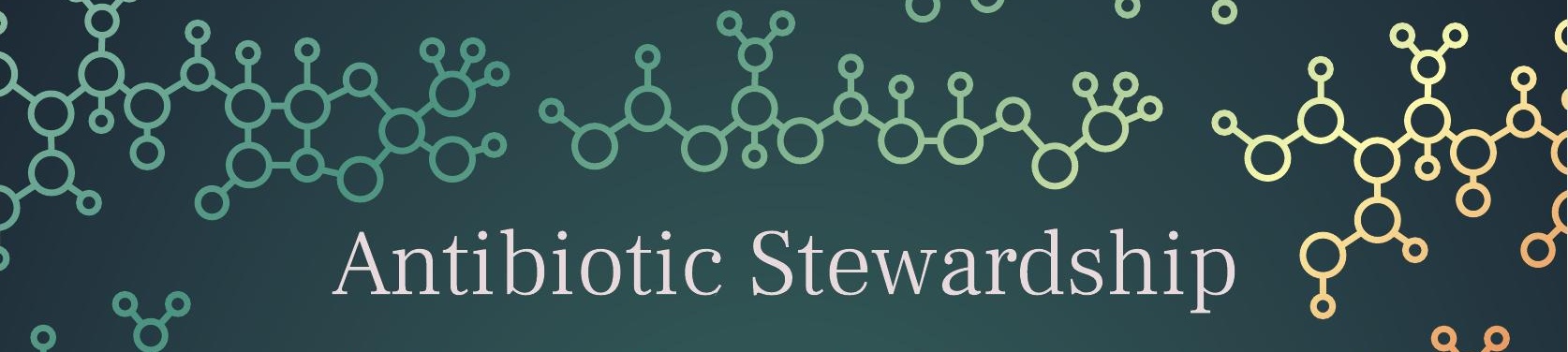

in Community-acquired Pneumonia

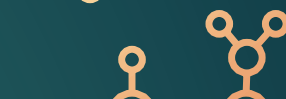

103

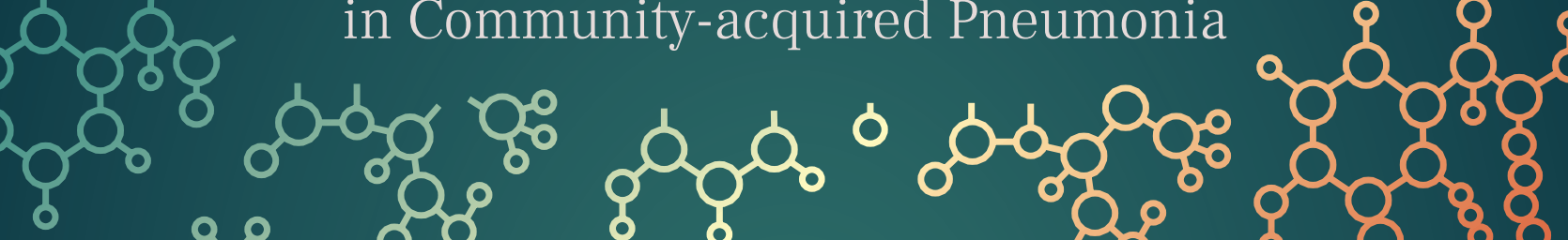

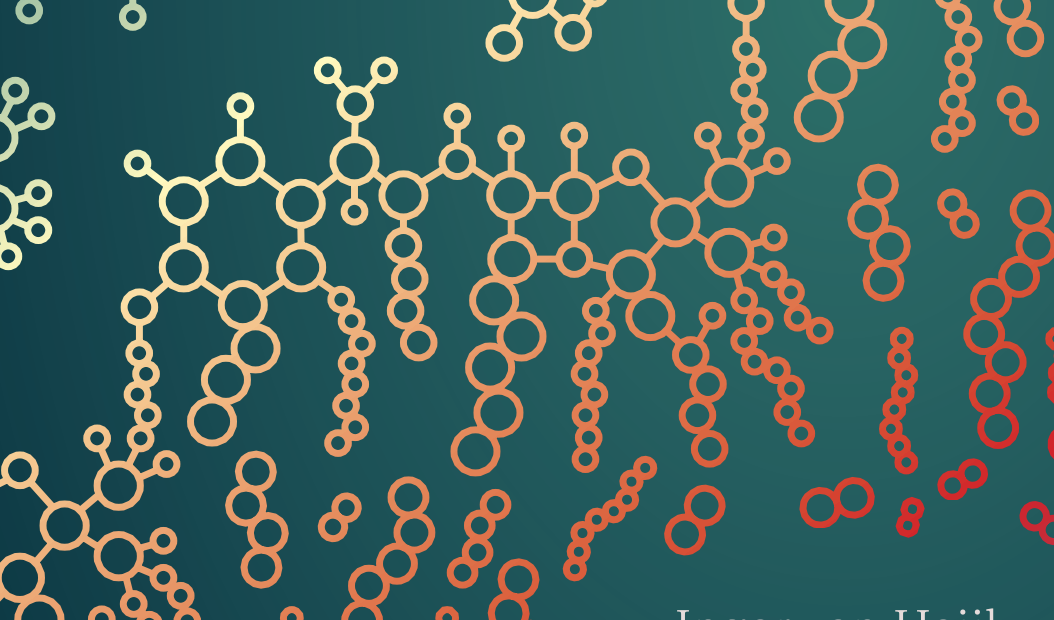

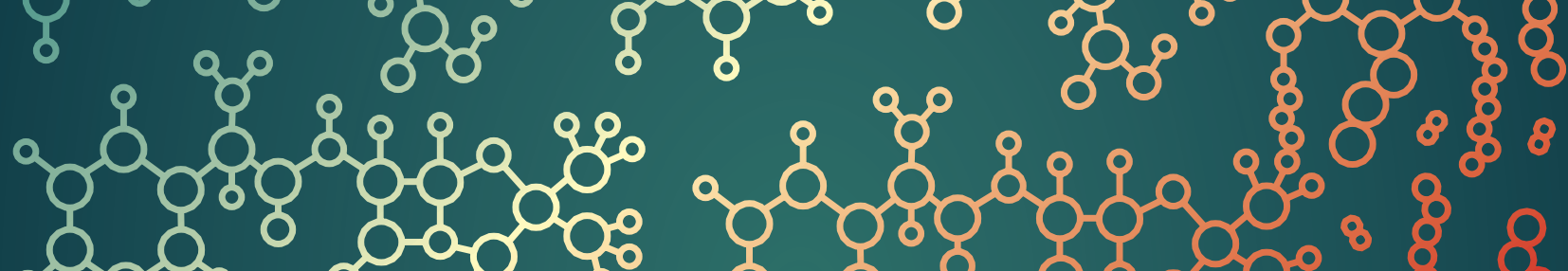
o o o o की की: $89: 98$ Inger van Heijl

8 88

8

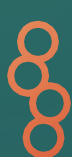





\section{Antibiotic stewardship in community-acquired pneumonia}




\section{Antibiotic stewardship in community-acquired pneumonia}

PhD thesis, Utrecht University, the Netherlands

ISBN:

978-94-6402-212-4

Author:

Inger van Heijl

Cover design:

Marthe Noordzij

Lay-out:

Ilse Modder I www.ilsemodder.nl

Printing:

Gildeprint I www.gildeprint.nl

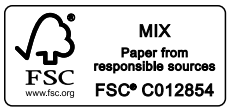

This thesis is printed with financial support from the Julius Center for Health Sciences and Primary care, Unirversity Medical Center Utrecht and from Tergooi hospital.

Copyright @ 2020 Inger van Heijl

All rights reserved. No part of this thesis may be reproduced, stored or transmitted in any form or by any means without the permission of the author. The copyright articles of the articles that have been published had been transferred to the respective publishers. 


\title{
Antibiotic stewardship in community-acquired pneumonia
}

\section{Antibiotic stewardship bij een thuis-opgelopen longontsteking}

(met een samenvatting in het Nederlands)

\author{
Proefschrift
}

\begin{abstract}
ter verkrijging van de graad van doctor aan de Universiteit Utrecht op gezag van de rector magnificus, prof. dr. H.R.B.M. Kummeling, ingevolge het besluit van het college voor promoties in het openbaar te verdedigen op
\end{abstract}

dinsdag 3 november 2020

des middags te 14.45 uur

door

Inger van Heijl

geboren op 22 augustus 1986

te Rheden 
Promotor:

Copromotoren:
Prof. dr. M.J.M. Bonten

Dr. P.D. van der Linden

Dr. J.W. Dorigo - Zetsma 


\section{CONTENTS}

Chapter 1. General introduction 9

PART I. ANTIBIOTIC STEWARDSHIP INTERVENTIONS 17

Chapter 2. Inappropriate Use of Antimicrobials for Lower Respiratory 19

Tract Infections in Elderly Patients: Patient- and

Community-Related Implications and Possible Interventions.

Chapter 3. Narrow-spectrum antibiotics for community-acquired 39

pneumonia in adults: a stepped-wedge cluster randomized

trial.

Chapter 4. Process evaluation of an effective antibiotic stewardship intervention in hospitalized patients with community-acquired pneumonia.

\section{PART II. METHODOLOGY USED IN ANTIBIOTIC STEWARDSHIP STUDIES}

Chapter 5. The quality of studies evaluating antimicrobial stewardship interventions: a systematic review.

Chapter 6. Confounding by indication of the safety of de-escalation in community-acquired pneumonia: A simulation study embedded in a prospective cohort.

Chapter 7. Safety of de-escalation of empirical therapy in terms of mortality: a literature review of study methodology and recommendations for observational studies.

\section{Chapter 8. Summary and Discussion}

Dutch summary (samenvatting in het Nederlands)

Contributing authors 200

Acknowledgements (dankwoord) 204

Curriculum vitae

List of publications 
abo

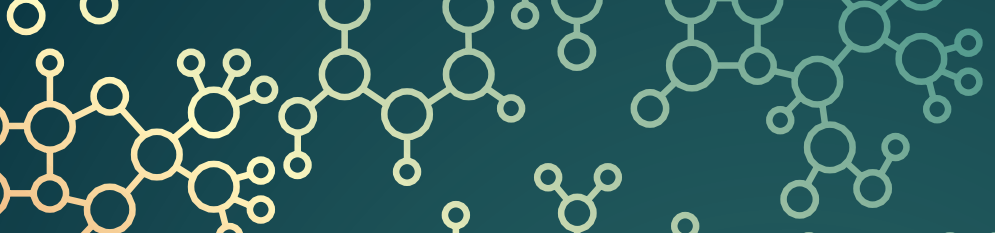
o

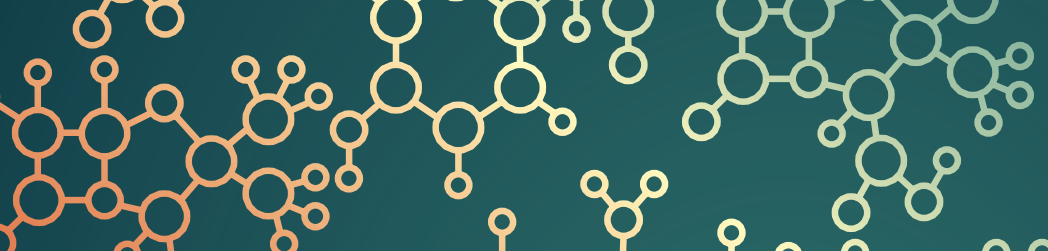

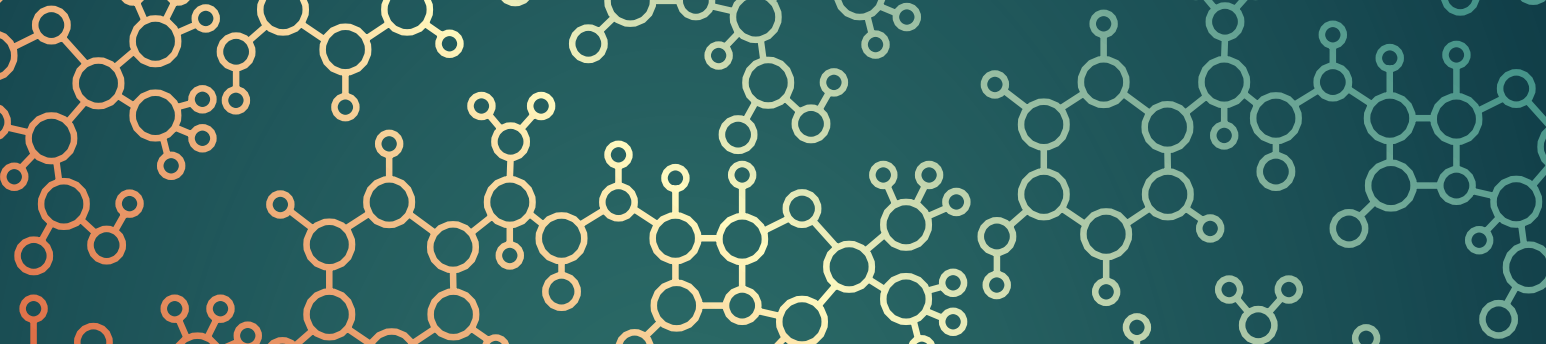

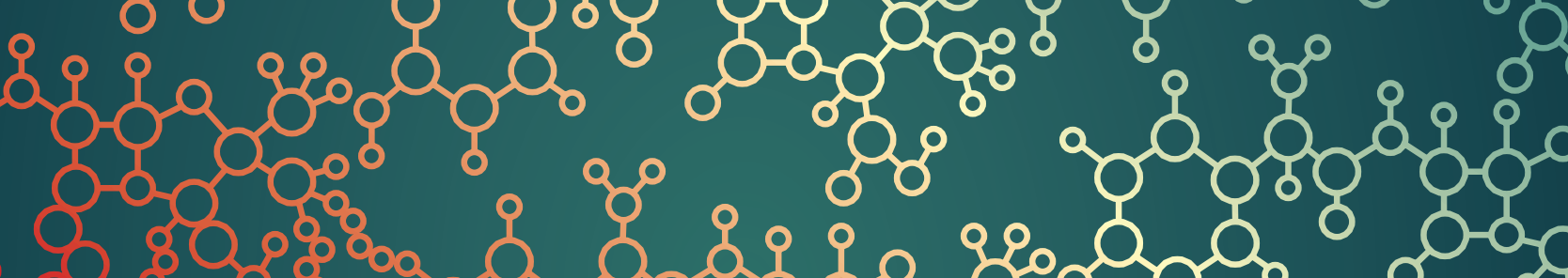
80\%

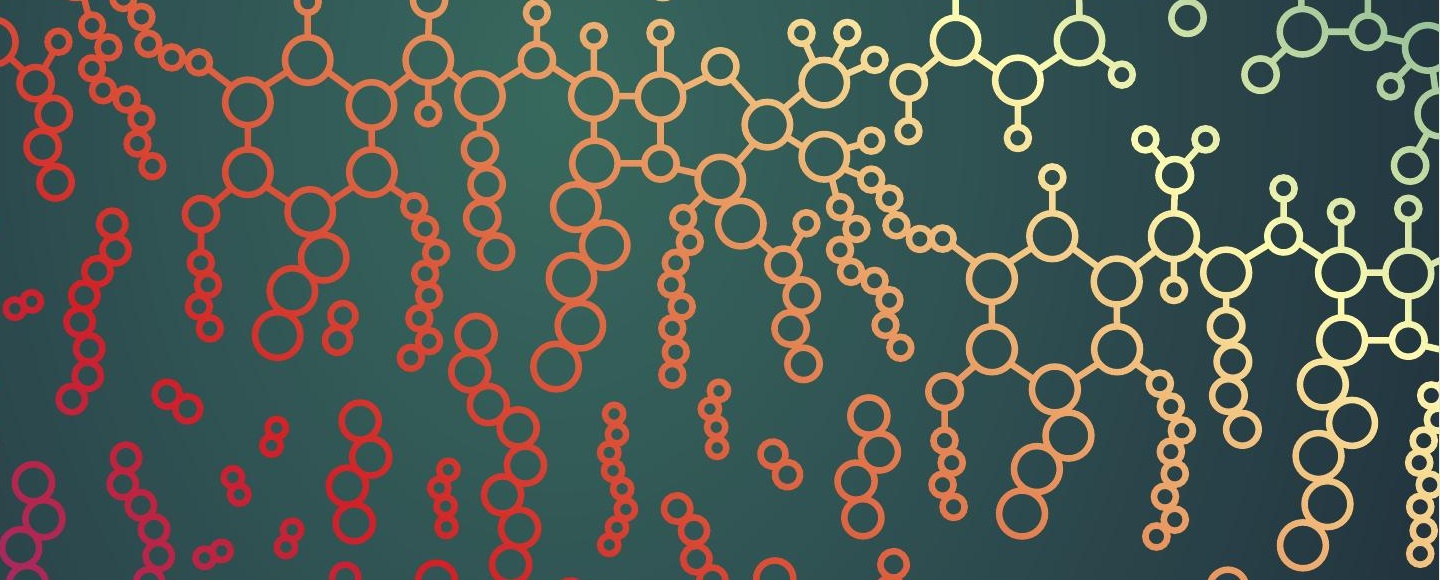
$88 \% 888$ $\circ 88$ \& 8

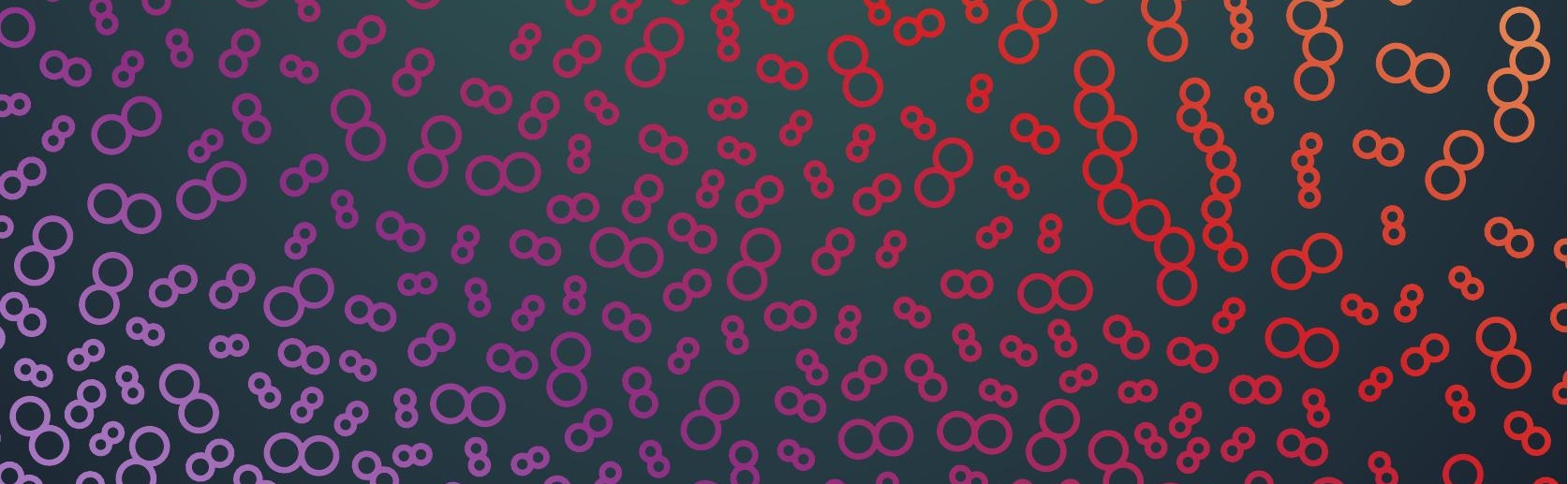


General introduction 


\section{GENERAL INTRODUCTION}

Community-acquired pneumonia (CAP) is a lower respiratory tract infection (LRTI), acquired outside the hospital. CAP is associated with a high morbidity and mortality, mainly in elderly patients ${ }^{[1,2]}$. Elderly are more susceptible to infections than younger adults ${ }^{[3,4]}$, which is reflected in a higher incidence of LRTIs and mortality increasing with age ${ }^{[5-7]}$. This is due to a higher prevalence of comorbidities and by age-related altered immune regulation called 'immunosenescence' ${ }^{[8]}$. Regardless of a patients' age, the most frequently detected causal pathogen of CAP is Streptococcus pneumoniae [9]. According to the Dutch guideline for management of CAP in adults, recommended empirical treatment is based on disease severity ${ }^{[10]}$, with three methods to classify CAP as either mild, moderate-severe, or severe. These methods are the Pneumonia Severity Index (PSI or Fine score), CURB-65 score or the pragmatic classification (treatment at home; admission to a general medical ward; admission to an Intensive Care Unit (ICU)). The guideline does not prefer any of these three methods, only advises to use one scoring method per hospital. The empirical treatment of mild and moderate-severe CAP in the Dutch guideline is mainly directed at S. pneumoniae. In adequate dosages this pathogen can be treated with penicillin (when there are no risk factors for other pathogens). Empirical treatment is often evaluated after 48-72 hours. This time frame is based on (1) reaching clinical stability within 72 hours and (2) availability of culture results ${ }^{[10-12]}$. Patients with severe pneumonia are clinically very ill, which leaves no room for 72 hours of inappropriate therapy. Moreover, these patients have a higher risk for other causal pathogens ${ }^{[10]}$, that may not be susceptible to penicillin. Therefore, for this population broad-spectrum antibiotics, such as fluoroquinolone monotherapy or a 3 th generation cephalosporin with ciprofloxacin, are recommended ${ }^{[10]}$. In daily clinical practice though, guideline adherence appears to be low, mainly due to unnecessary use of broad-spectrum antibiotics in patients treated for moderate-severe CAP ${ }^{[13]}$. Antibiotic stewardship programs (ASP) have shown to be effective tools to increase guideline adherence resulting in reductions of broad-spectrum antibiotic use and adverse drug reactions ${ }^{[14]}$.

\section{ANTIBIOTIC STEWARDSHIP PROGRAMS (ASP)}

In the Netherlands before 2015, appropriate antibiotic use was guided by education, the availability of national (and local) guidelines ${ }^{[15]}$, and feedback and consultation on culture results by medical microbiologists. However, in clinical practice these strategies appeared to be insufficient. Inappropriate use of (broad-spectrum) antibiotics may lead to antibiotic resistance, increased healthcare costs and complications ${ }^{[14]}$. The aim of ASP is to minimize unnecessary and inappropriate prescribing of antibiotics by physicians 
without compromising clinical outcomes on the individual patient level. Therefore, antibiotic stewardship teams (A-teams) are mandatory in Dutch hospitals since January $2015^{[15]}$. There are several guidelines with recommendations for development and implementation of ASP ${ }^{[16-18]}$. In the Dutch guideline 15 ASP strategies are strongly recommended, such as; prescribe empirical treatment according to the guideline in patients with CAP or change empirical antibiotics to pathogen-directed therapy (deescalation) as soon as microbiological results are available ${ }^{[18]}$. Unfortunately the quality of evidence for some of these recommendations is (very) low. In the Dutch ASP guideline most evidence is based on observational studies which often suffer of the consequences of confounding by indication. This may result from a physicians' decision to de-escalate empirical antibiotic therapy in one patient and not in another patient. For example, patients with de-escalation at day 3 are often sooner clinically stable, compared to patients in which empirical treatment is continued at day 3. It is unknown to what extent study results are influenced by confounding by indication in such studies. So, there is a need for clear recommendations for performing future observational studies evaluating ASP strategies and for more well performed randomized controlled trials.

\section{OUTLINE OF THE THESIS}

The aim of this thesis is to gain more insight into how treatment of patients with CAP can be improved by ASP strategies (Part I) and how the methodology used in ASP studies can be improved (Part II).

The first part of the thesis will focus on ASP interventions in patients with lower respiratory tract infections (LRTI) and specifically CAP. In Chapter $\mathbf{2}$ we will describe several aspects of antibiotic use for LRTIs in elderly patients, such as definitions of inappropriate antibiotic use, possible negative impact of antibiotic therapy, and how to optimize antibiotic use in elderly with ASP interventions. Chapter 3 focusses on optimizing antibiotic use in patients hospitalized with moderate-severe CAP. The main results of the "Community-Acquired Pneumonia increasing Protocol adherence by Antibiotic stewardship in a steppedwedge Cluster-randomized Trial" (CAP-PACT trial) are described. In Chapter $\mathbf{4}$ a process evaluation of the ASP is performed, which includes a description of the intervention as planned, the intervention as delivered, the actual exposure to the intervention and recommendations to improve the intervention components based on semi-structured interviews with participants.

The second part of the thesis is about methodology used in ASP studies. Many published studies in this field suffer from methodological limitations. In Chapter $\mathbf{5}$ a comprehensive systematic review of ASP strategies focusing on study design quality is described. In 
Chapter $\mathbf{6}$ and $\mathbf{7}$ we focus on one specific ASP strategy; de-escalation of empirical antibiotic therapy. Observational studies have demonstrated that de-escalation of antibiotic therapy is independently associated with lower mortality, but this may result from confounding by indication (due to a difference in clinical stability between patients with a de-escalation compared to patients in which empirical treatment is continued). In Chapter $\mathbf{6}$ a sensitivity analysis is described to quantify the potential confounding effect of clinical stability on the estimated impact of de-escalation on mortality in patients with CAP. Chapter 7 provides a review of the methodological limitations of observational studies evaluating the effects of de-escalation of empirical antibiotic therapy on mortality, with recommendations for future (observational) studies.

A summary of the main results of this thesis and a general discussion on antibiotic stewardship in patients with CAP is provided in Chapter $\mathbf{8}$. 


\section{REFERENCES}

1. E. Prina, O.T. Ranzani, A. Torres, Community-acquired pneumonia, Lancet (London, England) 386(9998) (2015) 1097-108.

2. R.G. Wunderink, G.W. Waterer, Clinical practice. Community-acquired pneumonia, N Engl J Med 370(6) (2014) 543-51.

3. K.C. Meyer, The role of immunity and inflammation in lung senescence and susceptibility to infection in the elderly, Semin Respir Crit Care Med 31(5) (2010) 561-74.

4. T.T. Yoshikawa, Epidemiology and unique aspects of aging and infectious diseases, Clin Infect Dis 30(6) (2000) 931-3.

5. J.P. Janssens, K.H. Krause, Pneumonia in the very old, The Lancet. Infectious diseases 4(2) (2004) 112-24.

6. E.R. Millett, J.K. Quint, L. Smeeth, R.M. Daniel, S.L. Thomas, Incidence of community-acquired lower respiratory tract infections and pneumonia among older adults in the United Kingdom: a population-based study, PLoS One 8(9) (2013) e75131.

7. T. Welte, A. Torres, D. Nathwani, Clinical and economic burden of community-acquired pneumonia among adults in Europe, Thorax 67(1) (2012) 71-9

8. G. Pawelec, Hallmarks of human "immunosenescence": adaptation or dysregulation?, Immun Ageing 9(1) (2012) 15.

9. D.F. Postma, C.H. van Werkhoven, L.J. van Elden, S.F. Thijsen, A.I. Hoepelman, J.A. Kluytmans, W.G. Boersma, C.J. Compaijen, E. van der Wall, J.M. Prins, J.J. Oosterheert, M.J. Bonten, Antibiotic treatment strategies for community-acquired pneumonia in adults, N Engl J Med 372(14) (2015) 1312-23.

10. W.J. Wiersinga, M.J. Bonten, W.G. Boersma, R.E. Jonkers, R.M. Aleva, B.J. Kullberg, J.A. Schouten, J.E. Degener, E.M.W. van de Garde, T.J. Verheij, A.P.E. Sachs, J.M. Prins, Management of community-acquired pneumonia in adults: 2016 guideline update from the Dutch Working Party on Antibiotic Policy (SWAB) and Dutch Association of Chest Physicians (NVALT), Neth J Med 76(1) (2018) 4-13.

11. E.A. Halm, M.J. Fine, T.J. Marrie, C.M. Coley, W.N. Kapoor, D.S. Obrosky, D.E. Singer, Time to clinical stability in patients hospitalized with community-acquired pneumonia: implications for practice guidelines, Jama 279(18) (1998) 1452-7.

12. M.S. Niederman, L.A. Mandell, A. Anzueto, J.B. Bass, W.A. Broughton, G.D. Campbell, N. Dean, T. File, M.J. Fine, P.A. Gross, F. Martinez, T.J. Marrie, J.F. Plouffe, J. Ramirez, G.A. Sarosi, A. Torres, R. Wilson, V.L. Yu, Guidelines for the management of adults with community-acquired pneumonia. Diagnosis, assessment of severity, antimicrobial therapy, and prevention, Am J Respir Crit Care Med 163(7) (2001) 1730-54.

13. S.M. Huijts, C.H. van Werkhoven, W.G. Boersma, J. Buijs, G. Buunk, C.J. Compaijen, L.J. van Elde, J.E. Gisolf, R. van der Kam, J.A. Kluytmans, B.A. Kuipers, J.J. Mager, B. Oppedijk, F. Palmen, J.M. Prins, B. van Reemst, M.H. Silbermann, F.H. van Tiel, E. van der Wall, T.S. van der Werf, M.J. Bonten, Guideline adherence for empirical treatment of pneumonia and patient outcome. Treating pneumonia in the Netherlands, Neth $J$ Med 71(10) (2013) 502-7.

14. P. Davey, C.A. Marwick, C.L. Scott, E. Charani, K. McNeil, E. Brown, I.M. Gould, C.R. Ramsay, S. Michie, 
Interventions to improve antibiotic prescribing practices for hospital inpatients, Cochrane Database Syst Rev 2 (2017) Cd003543.

15. The Dutch Working Party on AntibioticPolicy (SWAB), Antimicrobial Stewardship. <https://swab. nl>, 2016 (accessed January 30.2020).

16. T.F. Barlam, S.E. Cosgrove, L.M. Abbo, C. MacDougall, A.N. Schuetz, E.J. Septimus, A. Srinivasan, T.H. Dellit, Y.T. Falck-Ytter, N.O. Fishman, C.W. Hamilton, T.C. Jenkins, P.A. Lipsett, P.N. Malani, L.S. May, G.J. Moran, M.M. Neuhauser, J.G. Newland, C.A. OhI, M.H. Samore, S.K. Seo, K.K. Trivedi, Implementing an Antibiotic Stewardship Program: Guidelines by the Infectious Diseases Society of America and the Society for Healthcare Epidemiology of America, Clin Infect Dis 62(10) (2016) e51-77.

17. K. de With, F. Allerberger, S. Amann, P. Apfalter, H.R. Brodt, T. Eckmanns, M. Fellhauer, H.K. Geiss, O. Janata, R. Krause, S. Lemmen, E. Meyer, H. Mittermayer, U. Porsche, E. Presterl, S. Reuter, B. Sinha, R. Strauss, A. Wechsler-Fordos, C. Wenisch, W.V. Kern, Strategies to enhance rational use of antibiotics in hospital: a guideline by the German Society for Infectious Diseases, Infection 44(3) (2016) 395-439.

18. E.C. Schuts, M.E.J.L. Hulscher, J.W. Mouton, C.M. Verduin, J.W.T.C. Stuart, H.W.P.M. Overdiek, P.D. van der Linden, S. Natsch, C.M.P.M. Hertogh, T.F.W. Wolfs, J.A. Schouten, B.J. Kullberg, J.M. Prins, SWAB Guidelines for Antimicrobial Stewardship. <https://swab.nl/nl/antimicrobial-stewardship>, 2016). 



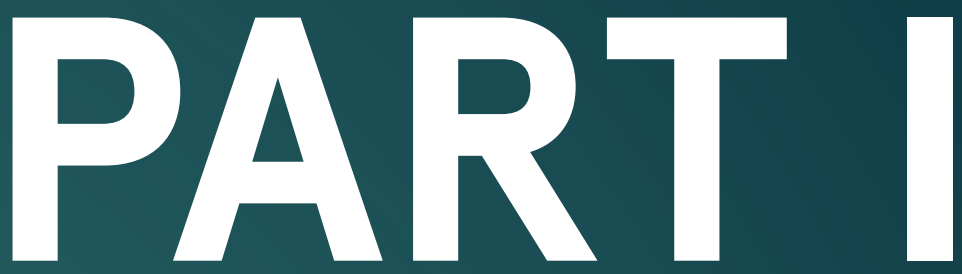

ANTIBIOTIC STEWARDSHIP INTERVENTIONS 
abo

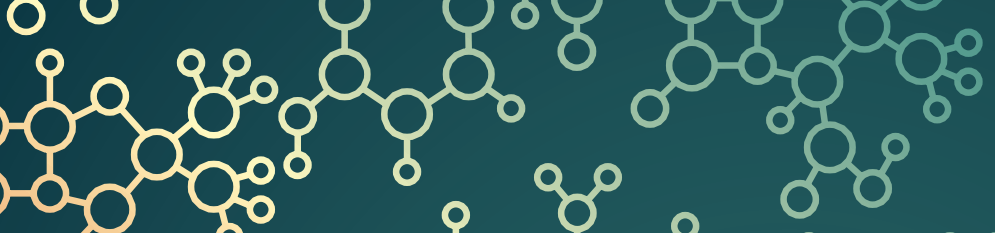
o

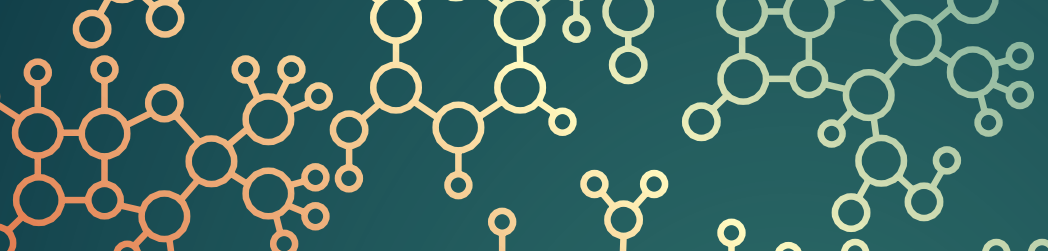

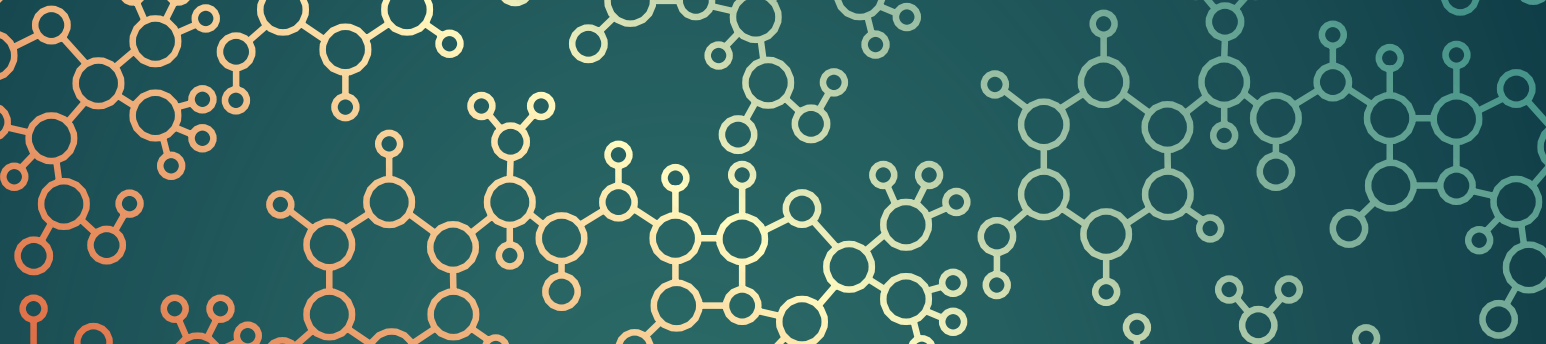

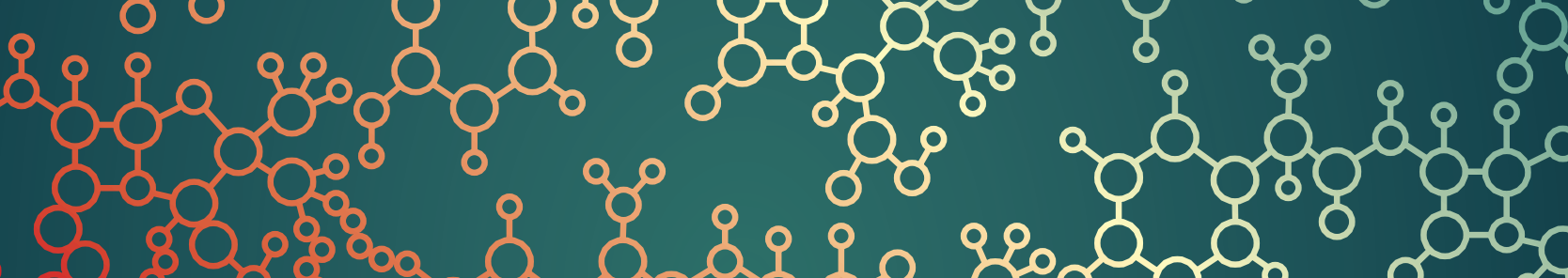
80\%

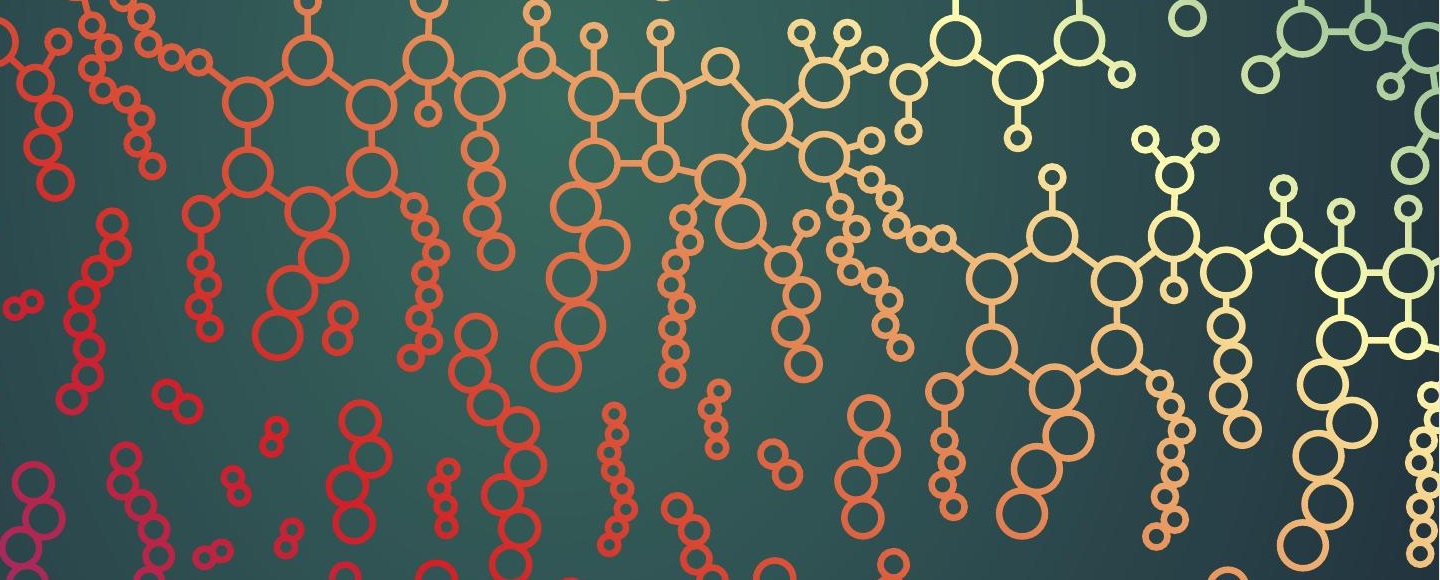
$88 \% 888$ $\circ 88$ \& 8

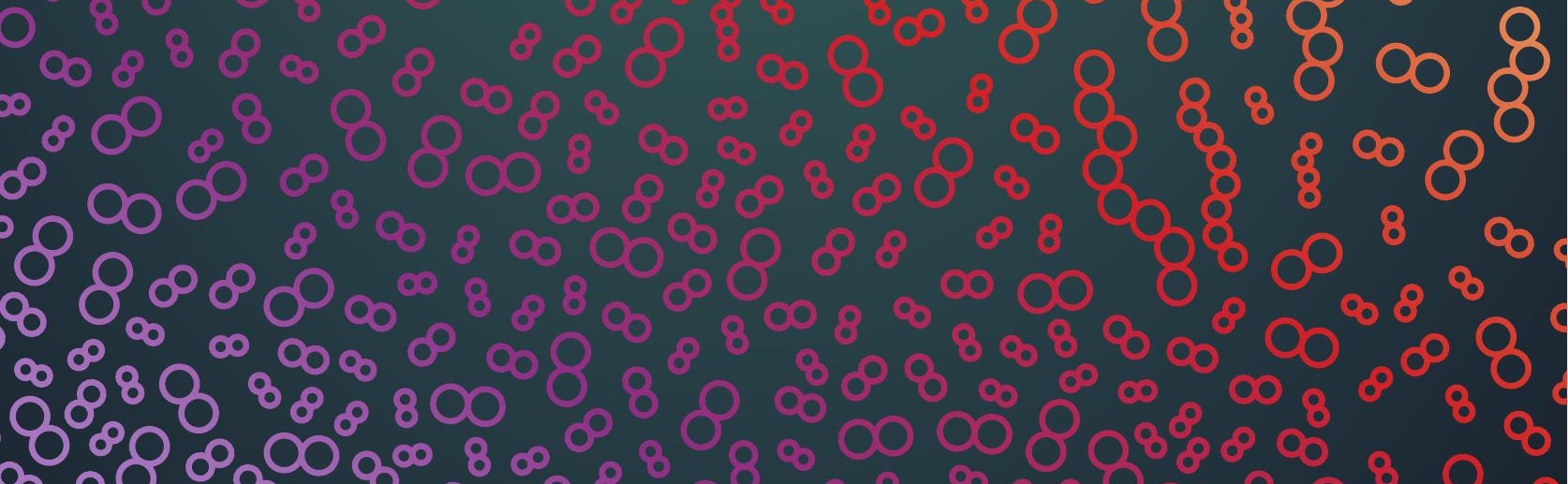




\section{Inappropriate Use of Antimicrobials for}

Lower Respiratory Tract Infections in Elderly Patients:

Patient- and CommunityRelated Implications and Possible Interventions.

Inger van Heijl, Valentijn A. Schweitzer, Lufang Zhang, Paul D. van der Linden, Cornelis H. van Werkhoven, Douwe F. Postma 


\section{ABSTRACT}

\section{BACKGROUND}

Elderly are more susceptible to infections which is reflected in the incidence and mortality of lower respiratory tract infections (LRTI) increasing with age. Several aspects of antimicrobial use for LRTIs in elderly patients should be considered to determine appropriateness.

\section{EXPERT OPINION/COMMENTARY}

We discuss possible differences in microbial aetiology between elderly and younger adults, definitions of inappropriate antimicrobial use for LRTIs currently found in the literature and their results, possible negative impact of antimicrobial therapy on an individual and community level, and finally, we propose that both antimicrobial stewardship interventions and novel rapid diagnostic techniques may optimize antimicrobial use in elderly patients with LRTI.

\section{KEY POINTS}

- Reports on (in)appropriate antimicrobial use lack a reference standard for defining and measuring appropriateness of treatment.

- Quinolones or macrolides should be restricted to selected cases empirically given the low incidence of atypical pathogens in elderly patients and higher risks of adverse drug events and drug-drug interactions.

- Use of low-dose CT scanning, point-of-care ultrasonography, or PoC-PCR testing for viral pathogens are promising research areas to decrease the inappropriate use of antimicrobials. 


\section{INTRODUCTION}

Elderly people (adults over 65 years of age) comprised a fifth of the total population

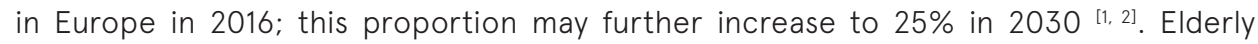
are more susceptible to infections and their sequelae than younger adults ${ }^{[3,4]}$. which is reflected in the incidence and mortality of lower respiratory tract infections (LRTIs) increasing with age ${ }^{[5-7]}$. Next to increased incidences of comorbidities, it is thought that age-related altered immune regulation, often referred to as 'immunosenescence', also contributes to this ${ }^{[8]}$, Several aspects of LRTIs in elderly make it increasingly difficult to determine the most appropriate antimicrobial therapy for individual patients. First, the aetiology of LRTI in elderly patients could be slightly different compared to younger adults, which would require adjusted empirical antimicrobial therapy. Also, diagnosis of LRTI in elderly could be more challenging which might lower the threshold for prescribing antimicrobials. Furthermore, with advancing age, the human body changes in composition and organ function, resulting in alteration of pharmacokinetics and pharmacodynamics of antimicrobials ${ }^{[9]}$. When combined with the increasing frequency of co-morbidities and/or polypharmacy, this facilitates the occurrence of adverse drug events (ADE) and drug-drug interactions.

We will discuss the aetiology, the currently used definitions for appropriate use of antimicrobials, and the different negative consequences of antimicrobial therapy in individual patients and the community for LRTIs in elderly patients. Finally, we propose targeted interventions to improve antimicrobial prescribing in these patients.

\section{MICROBIOLOGICAL AETIOLOGY}

Seven studies, all from Europe, have made head-to-head comparisons of aetiology in elderly and younger adult patients with LRTI ${ }^{[10-16]}$. A cut-off of 65 years of age was used to define categories. The ranges of the most commonly identified pathogens are summarized in Table 1. Streptococcus pneumoniae was the most frequently identified pathogen in both age groups. The most discernible differences between the two groups were that gram-negatives, especially Enterobacteriaceae, were found more frequent in elderly, whereas certain atypical pathogens (Legionella pneumophila, Mycoplasma pneumoniae, and Coxiella burnetti) were more frequent in younger patients. Increasing age and nursing-home residency are associated with colonization of gram-negative bacteria in the oropharynx. Micro-aspiration is considered an etiologic pathway in the development of LRTIs in elderly patients, which could explain the increase in gram- 
negatives ${ }^{[10,17,18]}$. The lower prevalence of atypical pathogens in elderly may be caused by less frequent exposure to risk factors ${ }^{[10]}$.

Table 1 Identified causal pathogen in studies with head to head comparison between younger and elderly patients

\begin{tabular}{llll}
\hline Pathogens & Young patients ${ }^{\text {a* }}$ & Elderly patients ${ }^{\text {** }}$ & References \\
\hline Streptococcus pneumoniae & $9-35 \%$ & $8.6-36 \%$ & {$[10-16]$} \\
Staphylococcus aureus & $0.3-4 \%$ & $0.0-5 \%$ & {$[11-13,15,16]$} \\
Haemophilus influenzae & $1-2 \%$ & $0.7-10 \%$ & {$[10-13,15,16]$} \\
Gram-negatives & $0-7 \%$ & $1.4-15 \%$ & {$[10-13,15]$} \\
Enterobacteraceae & $0.4 \%-1.3 \%$ & $0.9-2.6 \%$ & {$[13,16]$} \\
Atypical pathogens & $11-37 \%$ & $1-15 \%$ & {$[10,11,13-15]$} \\
$\quad$ Legionella pneumophila & $3.4 \%-5.2 \%$ & $1-5 \%$ & {$[10-16]$} \\
$\quad$ Mycoplasma pneumoniae & $2.8-15 \%$ & $0-3.2 \%$ & {$[10-16]$} \\
$\quad$ Coxiella burnetti & $0.7-15.8 \%$ & $0-3.5 \%$ & {$[10,13-15]$} \\
$\quad$ Chlamydia pneumoniae & $0.1-8.2 \%$ & $0-6.7 \%$ & {$[10,12-16]$} \\
Total viral pathogens & $3.6-4 \%$ & $4.5-13.4 \%$ & {$[10,11,14,15]$} \\
Influenza & $1.2-3.0 \%$ & $0.3-4.8 \%$ & {$[10,12,14-16]$} \\
Parainfluenza & $1.3 \%$ & $1-8.6 \%$ & {$[10,14]$} \\
RSV & $0.0-0.4 \%$ & $0.7-1.8 \%$ & {$[10,12,15]$} \\
Unknown & $24-79 \%$ & $40-80 \%$ & {$[10-16]$} \\
\hline
\end{tabular}

*Range of reported prevalence's of pathogens found in the literature. a: < 65 years, exception of van Vught et al. [11] < 50 years and Fernández Sabé et al. [10] has been excluded for this specific younger age group as their cutoff was 80 years. Otherwise this younger age group would also contain patients aged 65-80 years. b: $>65$ years, exceptions are Fernández Sabé et al. [10] > 80 years, Van Vught et al [11] > 80 years.

Higher incidences of viral pathogens in elderly patients have been reported, [12, 14 ${ }^{16]}$ although this was not demonstrated consistently [10, 11, 15]. Studies showing higher incidences of viral pathogens were mostly population based, including outpatients from general practitioners, while contradicting studies were hospital based, which may explain conflicting results. Also, bias could have been introduced because viral testing was not uniformly performed and age may have influenced the decision to test ${ }^{[19]}$. Two recent cohort studies of hospitalized community-acquired pneumonia (CAP) patients, one from the US $(N=2,259)$ and one from the Netherlands $(N=408)$ routinely performed testing and found a virus as the only pathogen in $22 \%$ and $13 \%$ of cases, respectively. In both studies the most common detected viral pathogens were human rhinovirus and influenza virus [20, 21].

The occurrence of a viral aetiology was similar for young and elderly patients in the US study ${ }^{[20]}$ The study from the Netherlands even found more viral pathogens with increasing age. ${ }^{[21]}$ The contribution of viral pathogens as a cause for CAP in all age groups might be larger than previously thought. 
Elderly patients more often had unknown aetiology in LRTI compared to younger adults $[10,11,13,15,16]$. Possible reasons for less detection of the causal pathogen could be a more conservative diagnostic approach in this group of patients or difficulty in obtaining good quality material for culturing ${ }^{[13]}$.

In recent years, Health-Care Associated Pneumonia (HCAP) has been proposed as a separate entity from CAP. HCAP may be more frequently caused by gram-negatives and MDRO, and is associated with higher mortality ${ }^{[22-25]}$. Yet, a meta-analysis of 24 studies concluded that HCAP criteria do not accurately predict MDRO, although low study quality and heterogeneous designs preclude a firm conclusion ${ }^{[22]}$. European studies tend to report community-like aetiology, while studies from Asia and the US show increased MDRO rates in HCAP patients ${ }^{[25-28]}$. To date, the clinical relevance of HCAP remains unclear ${ }^{[23,24]}$. In fact both IDSA and ESCMID guidelines do not address HCAP which leaves a gap in the recommendations regarding treatment for patients from the long-term care facilities (LTCFs).

The microbial aetiology does not justify routine empirical coverage of Legionella pneumophila as the low incidence is further decreased in elderly patients to around 1-5\% (Table 1). As members of our group have suggested before, beta-lactam monotherapy, preferably aminopenicillins, should generally be the first choice of empirical therapy. Naturally, the severity of disease, local epidemiologic data, prior cultures or known colonization of individual patients, comorbidities, or allergies could lead to another antibiotic choice. Doxycycline, the addition of a macrolide to beta-lactam therapy, or the newer $4^{\text {th }}$ generation fluoroquinolones are potent therapies, but higher risks of adverse drug events and drug-drug interactions should be considered.

\section{INAPPROPRIATE USE OF ANTIMICROBIALS}

Whether to start antimicrobial therapy for LRTI and choosing the specific class depends on multiple factors in daily clinical practice. Most factors in this decision pathway are dependent on clinical judgement, which interferes with evaluating appropriateness in a standardized way. Deviation from protocol for empirical therapy, definitive drug selection, and duration of therapy might be justifiable for individual patients for reasons that are not captured in guidelines.

These difficulties are also reflected in the different definitions of (in)appropriate antimicrobial therapy found in the literature. Some studies have assessed (in)appropriate 
antimicrobial therapy by evaluating empirical therapy and/or definitive drug selection through expert opinion ${ }^{[32-37]}$. Others have aimed at appropriateness of diagnosis, dosage, route of administration, or duration of antimicrobial therapy ${ }^{[38-42]}$. A less subjective method to assess appropriateness is to evaluate therapy according to in-vitro susceptibilities, yet this requires positive microbiological testing results and cannot be solely relied upon [32-35, 37]. Another method focuses on costs, defining inappropriateness as unnecessary use of combination therapy with same spectrum ${ }^{[43]}$. Lastly, the indication for starting antimicrobial therapy, i.e. unnecessary antimicrobial therapy, can be evaluated, where inappropriateness did seem to increase with age ${ }^{[44]}$. The (in)appropriateness criteria for several studies specifically addressing antimicrobials for LRTIs in elderly patients are summarized in Table 2. Generally, the proportion of appropriate antimicrobial treatment ranged from 60 to $80 \%$. Although none of these studies found an association with increasing age, it has been suggested that both nursing-home or LTCF residency and polypharmacy, all occurring more frequently with advancing age, increase the risk for inappropriate prescriptions ${ }^{[45]}$. For example, the point prevalence of antimicrobial use in nursing homes is about $10 \%$ and the proportion of prescribed antimicrobial courses deemed unnecessary or inappropriate after post-hoc review ranged from 20 to $75 \%$ [46-51].

It is clear that a reference standard for measuring inappropriate antimicrobial use is currently lacking, which was also concluded by a specific review of this subject ${ }^{[52]}$. More work on definitions and standardization is heavily needed to ensure evaluation of appropriateness will become more reliable and less dependent of the inter-observer variation inherent to expert evaluation ${ }^{[53]}$.

Although it has been suggested that certain potential inappropriate prescriptions could be automatically recognized by an electronic health records system today, we think this is unfeasible as human judgement is almost always needed ${ }^{[54]}$. For example, a patient with documented allergy to guideline recommended treatment could be automatically flagged by such a system as appropriate deviation, yet this is dependent on accurate interpretation and registration of allergy data, the latter often being incorrect ${ }^{[55]}$.

Despite the heterogeneity in definitions, and resulting heterogeneity in inappropriateness rates, there is a clear consensus on the adverse consequences of inappropriate antimicrobial use on the patient and community level ${ }^{[56,57]}$. Both will be addressed in the next section. 
Table 2 Examples from literature of different definitions of (in)appropriate antimicrobial use for LRTI in elderly.

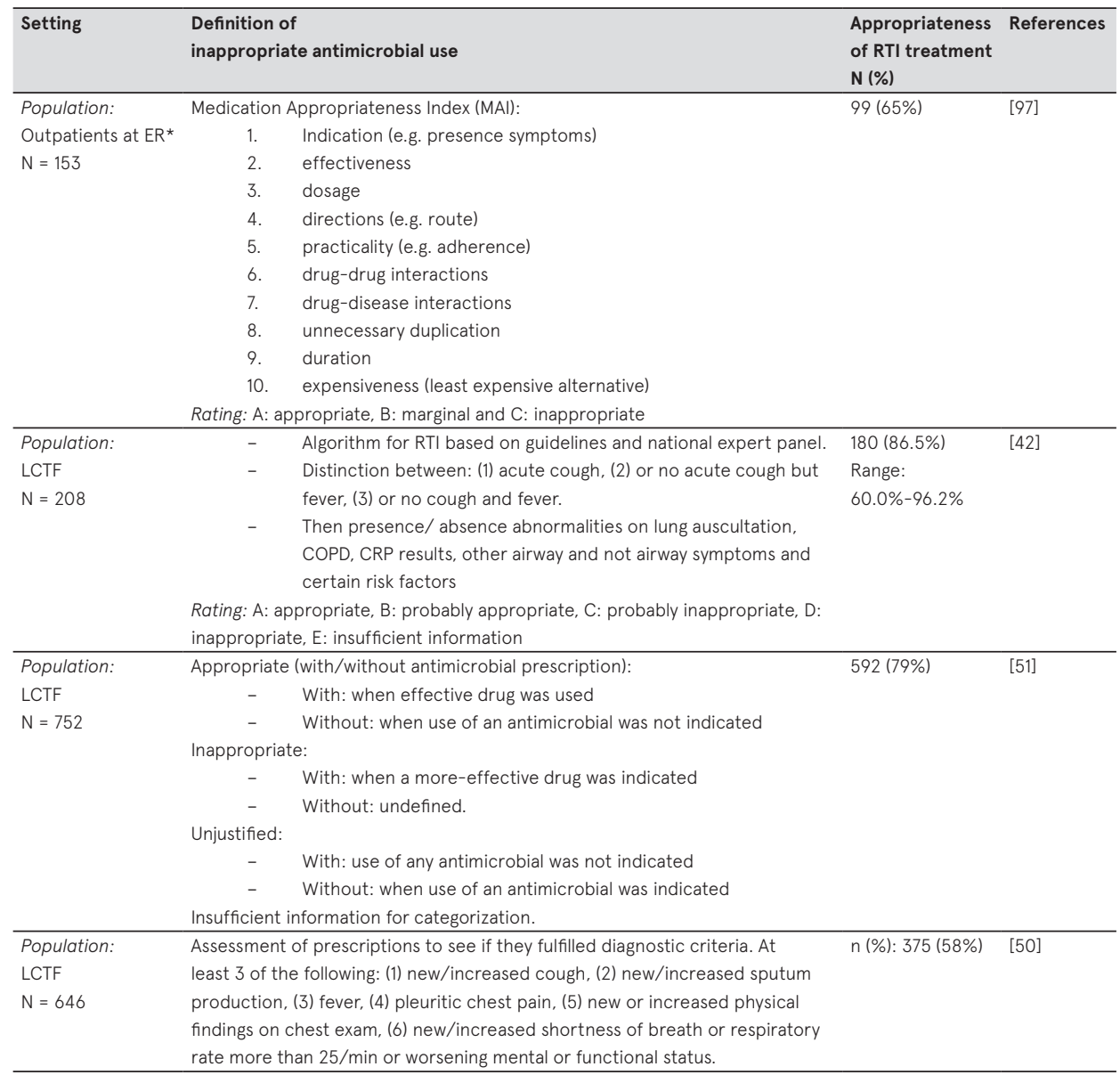

${ }^{\star} \mathrm{ER}=$ Emergency Room

\section{NEGATIVE IMPACT OF ANTIMICROBIALS IN ELDERLY}

Treating patients with antimicrobials is not without risk. Antimicrobials can have a negative impact on both individual users and the community. For individuals there is a risk of different adverse drug events (ADEs) including drug-drug interactions and drug-comorbidity interactions. In elderly patients, the age-related changes in pharmacokinetics, frequent concurrent use of medication, and higher prevalence of comorbidities, all contribute to an increased risk of such events. Faulkner et al. gives an extensive review of antimicrobial ADEs in elderly ${ }^{[58]}$. In the community, there is a risk of 
spread of Clostridium difficile infections (CDIs) and antimicrobial resistance.

\section{Adverse Drug Events (ADE)}

In a cohort study evaluating ADEs in hospitalized patients $(N=1488$; median age 59 years) $20 \%$ experienced at least one ADE, with gastro-intestinal, renal, and hematologic abnormalities being the most frequent. Furthermore, $20 \%$ of the reported antimicrobialrelated ADEs were due to unnecessary antimicrobial use ${ }^{[59]}$. In another cohort evaluating ADEs in nursing home residents, antimicrobials were the second most often reported drug-class to cause an ADE (20\%) after antipsychotics. The majority of observed ADEs were rashes and CDIs ${ }^{[60]}$.

Certain ADEs are serious enough that elderly patients have to visit the emergency room (ER). $10.6 \%$ of elderly patient ER visits was due to an ADE, with antimicrobials as one of the most frequently implicated medication classes $16.7 \%$ of all ADEs and $25 \%$ of definite or probable ADEs) [58, 61]. The most frequent (serious) ADEs in elderly who use beta-lactam antimicrobials for LRTI are drug fever, interstitial nephritis, and blood dyscrasias. For macrolides it is QT prolongation, and for trimethoprim-sulfamethoxazole

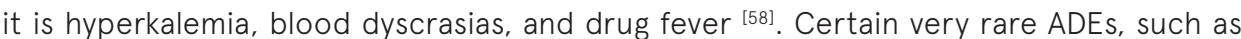
tendinitis and tendon rupture during fluoroquinolone use, are relatively more frequent in elderly and can be further increased by concomitant glucocorticoid use or renal failure ${ }^{[62,63]}$, although the absolute risks remain low ${ }^{[62] .}$

\section{Drug-drug interactions}

Elderly have an increased risk for drug-drug interactions since polypharmacy is more frequent ${ }^{[4]}$. It is estimated that $51 \%(1380 / 2707)$ of elderly patients receive $\geq 6$ drugs per day ${ }^{[64]}$. The most serious drug-drug interactions in elderly using antimicrobials for LRTIs are: trimethoprim-sulfamethoxazole in combination with vitamin $\mathrm{K}$ antagonists (increases anticoagulant effect), trimethoprim-sulfamethoxazole with potassium-sparing diuretics (risk of hospitalization due to hyperkaliemia), and clarithromycin/erythromycin with drugs which are deactivated by Cytochrome P450 3A4 enzymes (e.g. risk of rhabdomyolysis by increased concentrations of atorvastatin) ${ }^{[58]}$.

\section{Drug-comorbidity interactions}

Macrolides are associated with an increased risk for cardiovascular events and deaths; especially in (elderly) patients with a higher baseline risk for cardiovascular events ${ }^{\text {[65- }}$ 70]. These associations should caution the use of macrolides for LRTIs unless strictly necessary ${ }^{[9]}$. Renal insufficiency is more frequent in elderly, which results in a decreased elimination of certain (hydrophilic) antimicrobials (e.g. cephalosporin's, fluoroquinolones) 
and increases the risk of ADEs. Therefore, a dose adjustment is recommended for patients with impaired renal function ${ }^{[9,71]}$.

\section{Clostridium difficile infection}

Broad-spectrum antimicrobials disturb the gastrointestinal flora, contributing to an increased risk of CDI. Increased age, recent hospitalization, immune suppression, malignancy, chronic renal failure, and use of proton pump inhibitors have been identified as independent risk factors of CDI, and are highly prevalent in elderly ${ }^{[71,72]}$. Macrolides are more strongly associated with CDI than doxycycline, physicians may therefore choose for the latter when atypical coverage is deemed necessary ${ }^{[71]}$. Still, the risk of developing CDI with macrolides appears smaller than with fluoroquinolones, clindamycin, or broadspectrum beta-lactams ${ }^{[73]}$.

\section{Antimicrobial resistance}

There is clear consensus that inappropriate antimicrobial use contributes to antimicrobial resistance, potentially leading to increased morbidity, mortality, and health-care costs ${ }^{[74,75]}$. Antimicrobial resistance rates may increase with age, as reported in a Canadian surveillance study, further increasing risk for the elderly population. ${ }^{[76]}$. This could be explained by elderly people more often residing in LTCFs, needing hospitalization, receiving healthcare at home, and receiving antimicrobials, which are all risk factors for developing antimicrobial resistance ${ }^{[71,74,77-79]}$.

\section{OPTIMIZING APPROPRIATE ANTIMICROBIAL USE IN ELDERLY PATIENTS}

\section{Antimicrobial stewardship interventions}

Antimicrobial stewardship interventions aim at reducing inappropriate use of antimicrobials while maintaining good clinical outcomes. Elderly patients might especially benefit from antimicrobial stewardship as they may have the highest risk for worse clinical outcomes due to both over-treatment (e.g. antimicrobial side-effects, CDI) or under-treatment (e.g. infectious complications). The risk for and possible harm due to treatment differs per patient and depends on patient factors such as co-morbidities, immunological status, co-medication, previous antibiotic treatment, recent hospitalizations and the severity of the LRTI. Therefore, an individualized approach where individual patient risks are balanced to possible collateral damage in the form of selecting for antimicrobial resistance is recommended. 
Commonly used antimicrobial stewardship interventions include periodic or individual patient audit and feedback, decision support, education (educational meetings, educational materials), and formulary restriction of antimicrobials ${ }^{[74,80]}$.

To date, the majority of studies on stewardship interventions are performed on adult or pediatric patients, or on specific hospital settings e.g. ICU. In a recent systematic review of stewardship interventions in hospitalized patients, only $1.8 \%(4 / 211)$ of studies specifically targeted elderly patients ${ }^{[74,81-84]}$. Two controlled before-after studies showed a reduction in 30-day mortality after introducing a pneumonia guideline with clinical

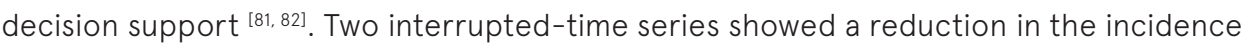
of $\mathrm{CDI}$ after implementation of an audit and feedback program and a restrictive antimicrobial policy ${ }^{[83,84]}$. Although promising, these four studies used non-randomized designs, risking confounding bias. There is a great need for high quality studies in elderly patients. Currently, the effects of antimicrobial stewardship interventions in elderly patients may be over- or underestimated.

As stated earlier the appropriate prescription of antimicrobials in LTCF is often impeded by the frailty of elderly residents, limited clinical evaluation -sometimes only consisting of nurse's assessment and telephone supervision by a physician, and lack of diagnostic testing. In nursing homes, the majority of antimicrobials are prescribed after telephone contact with nursing staff, highlighting the importance of involving nurses in antimicrobial stewardship programs ${ }^{[85]}$. In a recent review of stewardship interventions in LTCF, the following approaches were identified to be the most effective: multidisciplinary education; pre-prescriptive data collection tools; post-prescriptive prescriber recommendations; and introducing consultation by infectious diseases experts ${ }^{[86]}$. When designing antimicrobial stewardship interventions, it is important to consider the setting and corresponding prescription process. This was illustrated in nursing home interventions designed to improve communication which were only effective in reducing antimicrobial use when nursing staff was involved ${ }^{[87,88]}$. Appealing antimicrobial stewardship targets to improve antimicrobial use for LRTI in LTCF include improving the indication for starting antimicrobials, optimizing the use of available diagnostics, limiting the use of fluoroquinolones given their strong association with CDI, and ensuring proper dosing and duration of antimicrobial therapy ${ }^{[87,89]}$.

\section{New diagnostic techniques Imaging}

As infections can be difficult to diagnose in elderly patients, they may be initially treated with broad spectrum antimicrobials. Novel diagnostic techniques to establish the 
diagnosis of LRTI may particularly reduce unnecessary antimicrobials in elderly patients. The current cornerstone for the radiological diagnosis of pneumonia is the demonstration of an infiltrate by conventional chest $X$-ray. However, the estimated sensitivity of a chest $X$-ray is only $60-70 \%$ in patients with a clinical diagnosis of CAP $[31,90,91$. In addition, certain co-morbidities that are common in elderly patients (e.g. heart failure and chronic obstructive pulmonary disease) may impede the detection of pulmonary infiltrates ${ }^{[92]}$. The increasing availability of CT-scans and possibility of low- or ultra-low-dose scanning seems a promising alternative. Recently, CT-scans changed the diagnosis of $58.6 \%$ (95\% confidence interval, 53.2-64.0\%) of consecutive CAP patients, potentially leading to

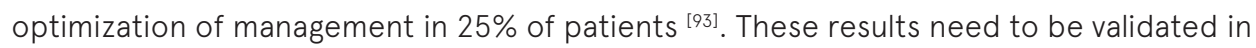
clinical practice to demonstrate improved patient outcomes while reducing antimicrobial use for non-infectious alternative diagnoses. In settings where CT-scans are not readily available, chest ultrasonography may be a valuable alternative. It has high diagnostic accuracy for pneumonia, can be done at the bedside, is highly reproducible in trained professionals, and costs are relatively low ${ }^{[94]}$.

\section{Microbiological testing}

Established microbiological techniques to determine the causative pathogen include respiratory cultures or PCR, blood cultures, and urinary antigen tests for Legionella and pneumococcus. However, the sensitivity of these tests are limited and in $60-70 \%$ of patients suspected of CAP no causative pathogen will be identified ${ }^{[20,31]}$.

When viral CAP is suspected, point-of-care PCR (PoC-PCR) for respiratory viruses might play a role in optimizing antimicrobial therapy. Previous studies using regular respiratory PCR have failed to show an effect on antimicrobial use, possibly because it is difficult to stop antimicrobials after they have been given for 1-2 days when PCR results become available ${ }^{[95,96]}$. PoC-PCR may allow withholding or rapid discontinuation of antimicrobials if a viral pathogen is identified, as results can be available in 1-2 hours. The effects and cost-effectiveness of PoC-PCR on antimicrobial use and patient outcome have not yet been investigated, but a cluster-randomized clinical trial evaluating the clinical effect of both low-dose CT and POC-PCR in patients admitted to non-ICU ward with CAP (ClinicalTrials.gov number, NCT01660204) is underway.

\section{CONCLUSION}

We have addressed several issues on the appropriate use of antimicrobials in elderly patients with LRTIs. As the microbial etiology is only slightly different compared to the 
younger population, the mainstay of treatment should consist of beta-lactam monotherapy. Extended coverage of gram-negatives could be considered in LTCF or nursing home residents. Quinolones or macrolides should be restricted to selected cases empirically given the low incidence of atypical pathogens in elderly patients and higher risks of ADEs and drug-drug interactions. Next to these adverse drug events, inappropriate use of antimicrobials contributes to CDI and antimicrobial resistance. A reference standard for measuring inappropriate antimicrobial use is currently lacking. Future work on definitions and standardization will hopefully increase validity and generalizability of reports on (in) appropriate antimicrobial therapy. Well-designed antimicrobial stewardship interventions could improve antimicrobial prescribing, but studies specifically targeting elderly patients are needed. These programs should generally consist of multiple components depending on the specific clinical setting, such as improving diagnostics (i.e. indication for starting antimicrobials) and ensuring proper dosing and duration of therapy. We argue that new diagnostic techniques such as low-dose CT-scanning or PoC-PCR testing for viral pathogens could potentially reduce inappropriate use of antimicrobials. Such techniques and interventions can hopefully decrease the inappropriate use of antimicrobials in the near future.

\section{List of abbreviations}

ADE, adverse drug events; CAP, community-acquired pneumonia; CDI, Clostridium difficile infection; ER, emergency room; HCAP, Health-Care Associated Pneumonia; LTCF, long-term care facility; LRTI, lower respiratory tract infections; PoC-PCR, point-of-care PCR.

\section{Acknowledgements}

None.

\section{Funding}

This research received no specific grant from any funding agency in the public, commercial, or not-for-profit sectors.

\section{Conflict of interests}

No conflict of interest to disclose. 


\section{REFERENCES}

1. United Nations, Department of Economic and Social Affairs, Population Division. World Population Ageing 2015. 2015;(ST/ESA/SER.A/390).

2. World Bank Group. Development Knowledge: Data on population ages 65 and above . In: . 2016. Accessed November 292017.

3. Meyer K. The role of immunity and inflammation in lung senescence and susceptibility to infection in the elderly. Semin Respir Crit Care Med. 2010; doi: 10.1055/s-0030-1265897.

4. Yoshikawa TT. Epidemiology and unique aspects of aging and infectious diseases. Clin Infect Dis. 2000; doi: $10.1086 / 313792$.

5. Millett ERC, Quint J, Smeeth L, Daniel R, Thomas S. Incidence of community-acquired lower respiratory tract infections and pneumonia among older adults in the United Kingdom: a population-based study. PLoS ONE. 2013; doi: 10.1371/journal.pone.0075131.

6. Welte T, Torres A, Nathwani D. Clinical and economic burden of community-acquired pneumonia among adults in Europe. Thorax. 2012; doi: 10.1136/thx.2009.129502.

7. Janssens J, Krause K. Pneumonia in the very old. Lancet Infect Dis. 2004; doi: 10.1016/S1473-3099(04)009314.

8. Pawelec G. Hallmarks of human "immunosenescence": adaptation or dysregulation?. Immun Ageing. 2012; doi: 10.1186/1742-4933-9-15.

9. Sera L, McPherson M. Pharmacokinetics and pharmacodynamic changes associated with aging and implications for drug therapy. Clin Geriatr Med. 2012; doi: 10.1016/j.cger.2012.01.007.

10. Fernández Sabé N, Carratalà J, Rosón B, Dorca J, Verdaguer R, Manresa F, Gudiol F. Community-acquired pneumonia in very elderly patients: causative organisms, clinical characteristics, and outcomes. Medicine (Baltimore). 2003; doi: 10.1097/01.md.0000076005.64510.87.

11. van Vught LA, Endeman H, Meijvis SC, Zwinderman AH, Scicluna BP, Biesma DH, van der Poll T. The effect of age on the systemic inflammatory response in patients with community-acquired pneumonia. Clin Microbiol Infect. 2014; doi: 10.1111/1469-0691.12717.

12. Kothe H, BauerT, Marre R, Suttorp N, Welte T, Dalhoff K. Outcome of community-acquired pneumonia: influence of age, residence status and antimicrobial treatment. Eur Respir J. 2008; doi: 10.1183/09031936.00092507.

13. Sahuquillo Arce J, Menéndez R, Méndez R, Amara Elori I, Zalacain R, Capelastegui A, Aspa J, Borderías L, Martín Villasclaras J, Bello S, Alfageme I, de Castro F, Rello J, Molinos L, Ruiz Manzano J, Torres A. Agerelated risk factors for bacterial aetiology in community-acquired pneumonia. Respirology. 2016; doi: 10.1111/ resp. 12851.

14. Capelastegui A, España P, Bilbao A, Gamazo J, Medel F, Salgado J, Gorostiaga I, Lopez de Goicoechea, Maria Jose, Gorordo I, Esteban C, Altube L, Quintana J. Etiology of community-acquired pneumonia in a population-based study: link between etiology and patients characteristics, process-of-care, clinical evolution and outcomes. BMC Infect Dis. 2012; doi: 10.1186/1471-2334-12-134.

15. Gutiérrez F, Masiá M, Rodríguez JC, Mirete C, Soldán B, Padilla S, Hernández I, De Ory F, Royo G, Hidalgo 
AM. Epidemiology of community-acquired pneumonia in adult patients at the dawn of the 21st century: a prospective study on the Mediterranean coast of Spain. Clin Microbiol Infect. 2005; doi: 10.1111/j.14690691.2005.01226.x

16. Klapdor B, Ewig S, Pletz M, Rohde G, Schütte H, Schaberg T, Welte T. Community-acquired pneumonia in younger patients is an entity on its own. Eur Respir J. 2012; doi: 10.1183/09031936.00110911.

17. Marik PE. Aspiration pneumonitis and aspiration pneumonia. N Engl J Med. 2001; doi: 10.1056/ NEJM200103013440908.

18. Reza Shariatzadeh M, Huang J, Marrie T. Differences in the features of aspiration pneumonia according to site of acquisition: community or continuing care facility. J Am Geriatr Soc. 2006; doi: 10.1111/j.15325415.2005.00608.x.

19. Moons KGM, Royston P, Vergouwe Y, Grobbee D, Altman D. Prognosis and prognostic research: what, why, and how?. BMJ. 2009; doi: 10.1136/bmj.b375.

20. Jain S, Self W, Wunderink R, Fakhran S, Balk R, Bramley A, Reed C, Grijalva C, Anderson E, Courtney DM, Chappell J, Qi C, Hart E, Carroll F, Trabue C, Donnelly H, Williams D, Zhu Y, Arnold S, Ampofo K, Waterer G, Levine M, Lindstrom S, Winchell J, Katz J, Erdman D, Schneider E, Hicks L, McCullers J, Pavia A, Edwards K, Finelli L. Community-Acquired Pneumonia Requiring Hospitalization among U.S. Adults. N Engl J Med. 2015; doi: 10.1056/NEJMoa1500245

21. Huijskens EGW, Koopmans M, Palmen FMH, van Erkel, Adriana J M, Mulder PGH, Rossen JWA. The value of signs and symptoms in differentiating between bacterial, viral and mixed aetiology in patients with communityacquired pneumonia. J Med Microbiol. 2014; doi: 10.1099/jmm.0.067108-0.

22. Chalmers J, Rother C, Salih W, Ewig S. Healthcare-associated pneumonia does not accurately identify potentially resistant pathogens: a systematic review and meta-analysis. Clin Infect Dis. 2014; doi: 10.1093/ cid/cit734.

23. José R, Periselneris J, Brown J. Community-acquired pneumonia. Curr Opin Pulm Med. 2015; doi: 10.1097/ MCP.0000000000000150.

24. Montravers P, Harpan A, Guivarch E. Current and Future Considerations for the Treatment of HospitalAcquired Pneumonia. Adv Ther. 2016; doi: 10.1007/s12325-016-0293-x.

25. Lopez A, Amaro R, Polverino E. Does health care associated pneumonia really exist?. Eur J Intern Med. 2012; doi: 10.1016/j.ejim.2012.05.006.

26. Ewig S, Klapdor B, Pletz M, Rohde G, Schütte H, Schaberg T, Bauer T, Welte T. Nursing-home-acquired pneumonia in Germany: an 8-year prospective multicentre study. Thorax. 2012; doi: 10.1136/thoraxjnl-2011-200630.

27. Lim WS, Macfarlane JT. A prospective comparison of nursing home acquired pneumonia with community acquired pneumonia. Eur Respir J. 2001.

28. Polverino E, Dambrava P, Cillóniz C, Balasso V, Marcos MA, Esquinas C, Mensa J, Ewig S, Torres A. Nursing home-acquired pneumonia: a 10 year single-centre experience. Thorax. 2010; doi: 10.1136/thx.2009.124776.

29. Eliakim Raz N, Robenshtok E, Shefet D, Gafter Gvili A, Vidal L, Paul M, Leibovici L. Empiric antibiotic coverage of atypical pathogens for community-acquired pneumonia in hospitalized adults. Cochrane Database Syst Rev. 2012; doi: 10.1002/14651858.CD004418.pub4 
30. Mills G, Oehley M, Arrol B. Effectiveness of beta lactam antibiotics compared with antibiotics active against atypical pathogens in non-severe community acquired pneumonia: meta-analysis. BMJ. 2005; doi: 10.1136/ bmj.38334.591586.82.

31. Postma D, van Werkhoven C, van Elden, Leontine J R, Thijsen SFT, Hoepelman AIM, Kluytmans, Jan A J W, Boersma W, Compaijen C, van der Wall E, Prins J, Oosterheert J, Bonten MJM. Antibiotic treatment strategies for community-acquired pneumonia in adults. N Engl J Med. 2015; doi: 10.1056/NEJMoa1406330.

32. Kollef MH, Sherman G, Ward S, Fraser VJ. Inadequate antimicrobial treatment of infections: a risk factor for hospital mortality among critically ill patients. Chest. 1999.

33. Micek S, Lloyd A, Ritchie D, Reichley R, Fraser V, Kollef M. Pseudomonas aeruginosa bloodstream infection: importance of appropriate initial antimicrobial treatment. Antimicrob Agents Chemother. 2005; doi: 10.1128/ AAC.49.4.1306-1311.2005.

34. Fraser A, Paul M, Almanasreh N, Tacconelli E, Frank U, Cauda R, Borok S, Cohen M, Andreassen S, Nielsen A, Leibovici L. Benefit of appropriate empirical antibiotic treatment: thirty-day mortality and duration of hospital stay. Am J Med. 2006; doi: 10.1016/j.amjmed.2006.03.034.

35. Ciccolini M, Spoorenberg V, Geerlings SE, Prins JM, Grundmann H. Using an index-based approach to assess the population-level appropriateness of empirical antibiotic therapy. J Antimicrob Chemother. 2015; doi: $10.1093 / \mathrm{jac} / \mathrm{dku} 336$.

36. Seaton RA, Nathwani D, Burton P, McLaughlin C, MacKenzie AR, Dundas S, Ziglam H, Gourlay Y, Beard K, Douglas E. Point prevalence survey of antibiotic use in Scottish hospitals utilising the Glasgow Antimicrobial Audit Tool (GAAT). Int J Antimicrob Agents. 2007; doi: 10.1016/j.ijantimicag.2006.10.020.

37. Kumar A, Ellis P, Arabi Y, Roberts D, Light B, Parrillo J, Dodek P, Wood G, Simon D, Peters C, Ahsan M, Chateau D. Initiation of inappropriate antimicrobial therapy results in a fivefold reduction of survival in human septic shock. Chest. 2009; doi: 10.1378/chest.09-0087.

38. Raveh D, Levy Y, Schlesinger Y, Greenberg A, Rudensky B, Yinnon AM. Longitudinal surveillance of antibiotic use in the hospital. QJM. 2001.

39. James R, Upjohn L, Cotta M, Luu S, Marshall C, Buising K, Thursky K. Measuring antimicrobial prescribing quality in Australian hospitals: development and evaluation of a national antimicrobial prescribing survey tool. J Antimicrob Chemother. 2015; doi: 10.1093/jac/dkv047.

40. Vlahovic Palcevski V, Francetic I, Palcevski G, Novak S, Abram M, Bergman U. Antimicrobial use at a university hospital: appropriate or misused? A qualitative study. Int J Clin Pharmacol Ther. 2007.

41. Peron E, Hirsch A, Jury L, Jump RLP, Donskey C. Another setting for stewardship: high rate of unnecessary antimicrobial use in a veterans affairs long-term care facility. J Am Geriatr Soc. 2013; doi: 10.1111/jgs.12099.

42. van Buul L, Veenhuizen R, Achterberg W, Schellevis F, Essink, Rob T G M, de Greeff S, Natsch S, van der Steen, Jenny T, Hertogh CMPM. Antibiotic prescribing in Dutch nursing homes: how appropriate is it?. J Am Med Dir Assoc. 2015; doi: 10.1016/j.jamda.2014.10.003.

43. Schultz L, Lowe T, Srinivasan A, Neilson D, Pugliese G. Economic impact of redundant antimicrobial therapy in US hospitals. Infect Control Hosp Epidemiol. 2014; doi: 10.1086/678066.

44. Willemsen I, van der Kooij T, van Benthem B, Wille J, Kluytmans J. Appropriateness of antimicrobial therapy: 
a multicentre prevalence survey in the Netherlands, 2008-2009. Euro Surveill. 2010.

45. Hajjar E, Cafiero A, Hanlon J. Polypharmacy in elderly patients. Am J Geriatr Pharmacother. 2007; doi: 10.1016/j.amjopharm.2007.12.002.

46. Warren JW, Palumbo FB, Fitterman L, Speedie SM. Incidence and characteristics of antibiotic use in aged nursing home patients. J Am Geriatr Soc. 1991

47. Rotjanapan P, Dosa D, Thomas K. Potentially inappropriate treatment of urinary tract infections in two Rhode Island nursing homes. Arch Intern Med. 2011; doi: 10.1001/archinternmed.2011.13

48. Zimmer JG, Bentley DW, Valenti WM, Watson NM. Systemic antibiotic use in nursing homes. A quality assessment. J Am Geriatr Soc. 1986.

49. Pickering TD, Gurwitz JH, Zaleznik D, Noonan JP, Avorn J. The appropriateness of oral fluoroquinoloneprescribing in the long-term care setting. J Am Geriatr Soc. 1994.

50. Loeb M, Simor AE, Landry L, Walter S, McArthur M, Duffy J, Kwan D, McGeer A. Antibiotic use in Ontario facilities that provide chronic care. J Gen Intern Med. 2001.

51. Vergidis P, Hamer D, Meydani S, Dallal G, Barlam T. Patterns of antimicrobial use for respiratory tract infections in older residents of long-term care facilities. J Am Geriatr Soc. 2011; doi: 10.1111/j.1532-5415.2011.03406.x.

52. Spivak E, Cosgrove S, Srinivasan A. Measuring Appropriate Antimicrobial Use: Attempts at Opening the Black Box. Clin Infect Dis. 2016; doi: 10.1093/cid/ciw658

53. Casaroto E, Marra A, Camargo TZS, de Souza, Ana Rita Araújo, de Almeida, Carlos Eduardo Saldanha, Pedroti E, Victor EdS, dos Santos, Oscar Fernando Pavão, Edmond M, Campos A. Agreement on the prescription of antimicrobial drugs. BMC Infect Dis. 2015; doi: 10.1186/s12879-015-0992-y.

54. Baclet N, Ficheur G, Alfandari S, Ferret L, Senneville E, Chazard E, Beuscart J. Explicit definitions of potentially inappropriate prescriptions of antibiotics in older patients: a compilation derived from a systematic review. Int J Antimicrob Agents. 2017; doi: 10.1016/j.ijantimicag.2017.08.011

55. Sogn DD, Evans R, Shepherd GM, Casale TB, Condemi J, Greenberger PA, Kohler PF, Saxon A, Summers RJ, VanArsdel PP. Results of the National Institute of Allergy and Infectious Diseases Collaborative Clinical Trial to test the predictive value of skin testing with major and minor penicillin derivatives in hospitalized adults Arch Intern Med. 1992

56. Barnes S, Rock C, Harris A, Cosgrove S, Morgan D, Thom K. The Impact of Reducing Antibiotics on the Transmission of Multidrug-Resistant Organisms. Infect Control Hosp Epidemiol. 2017; doi: 10.1017/ice.2017.34.

57. Barlam T, Cosgrove S, Abbo L, MacDougall C, Schuetz A, Septimus E, Srinivasan A, Dellit T, Falck Ytter Y, Fishman N, Hamilton C, Jenkins T, Lipsett P, Malani P, May L, Moran G, Neuhauser M, Newland J, Ohl C, Samore M, Seo S, Trivedi K. Implementing an Antibiotic Stewardship Program: Guidelines by the Infectious Diseases Society of America and the Society for Healthcare Epidemiology of America. Clin Infect Dis. 2016: doi: $10.1093 /$ cid/ciw118.

58. Faulkner C, Cox H, Williamson J. Unique aspects of antimicrobial use in older adults. Clin Infect Dis. 2005; doi: $10.1086 / 428125$.

59. Tamma P, Avdic E, Li D, Dzintars K, Cosgrove S. Association of Adverse Events With Antibiotic Use in Hospitalized Patients. JAMA Intern Med. 2017; doi: 10.1001/jamainternmed.2017.1938. 
60. Gurwitz JH, Field TS, Avorn J, McCormick D, Jain S, Eckler M, Benser M, Edmondson AC, Bates DW. Incidence and preventability of adverse drug events in nursing homes. Am J Med. 2000.

61. Hohl CM, Dankoff J, Colacone A, Afilalo M. Polypharmacy, adverse drug-related events, and potential adverse drug interactions in elderly patients presenting to an emergency department. Ann Emerg Med. 2001; doi: 10.1067/mem.2001.119456.

62. van der Linden, Paul D, Sturkenboom, Miriam C J M, Herings RMC, Leufkens HMG, Rowlands S, Stricker BHC. Increased risk of achilles tendon rupture with quinolone antibacterial use, especially in elderly patients taking oral corticosteroids. Arch Intern Med. 2003; doi: 10.1001/archinte.163.15.1801.

63. Wise B, Peloquin C, Choi H, Lane N, Zhang Y. Impact of age, sex, obesity, and steroid use on quinoloneassociated tendon disorders. Am J Med. 2012; doi: 10.1016/j.amjmed.2012.05.027.

64. Fialová D, Topinková E, Gambassi G, Finne Soveri H, Jónsson P, Carpenter I, Schroll M, Onder G, Sørbye L, Wagner C, Reissigová J, Bernabei R. Potentially inappropriate medication use among elderly home care patients in Europe. JAMA. 2005; doi: 10.1001/jama.293.11.1348.

65. Ray W, Murray K, Hall K, Arbogast P, Stein CM. Azithromycin and the risk of cardiovascular death. N Engl J Med. 2012; doi: 10.1056/NEJMoa1003833.

66. Ray W, Murray K, Meredith S, Narasimhulu S, Hall K, Stein CM. Oral erythromycin and the risk of sudden death from cardiac causes. N Engl J Med. 2004; doi: 10.1056/NEJMoa040582.

67. Mortensen E, Halm E, Pugh M, Copeland L, Metersky M, Fine M, Johnson C, Alvarez C, Frei C, Good C, Restrepo M, Downs J, Anzueto A. Association of azithromycin with mortality and cardiovascular events among older patients hospitalized with pneumonia. JAMA. 2014; doi: 10.1001/jama.2014.4304.

68. Schembri S, Williamson P, Short P, Akram A, Taylor J, Singanayagam A, Hill A, Chalmers J. Cardiovascular events after clarithromycin use in lower respiratory tract infections: analysis of two prospective cohort studies. BMJ. 2013; doi: 10.1136/bmj.f1235.

69. Chou H, Wang J, Chang C, Lai C, Lai M, Chan KA. Risks of cardiac arrhythmia and mortality among patients using new-generation macrolides, fluoroquinolones, and B-lactam/B-lactamase inhibitors: a Taiwanese nationwide study. Clin Infect Dis. 2015; doi: 10.1093/cid/ciu914.

70. Svanström H, Pasternak B, Hviid A. Use of azithromycin and death from cardiovascular causes. N Engl J Med. 2013; doi: 10.1056/NEJMoa1300799

71. Petrosillo N, Cataldo M, Pea F. Treatment options for community-acquired pneumonia in the elderly people. Expert Rev Anti Infect Ther. 2015; doi: 10.1586/14787210.2015.1021783.

72. Asempa T, Nicolau D. Clostridium difficile infection in the elderly: an update on management. Clin Interv Aging. 2017; doi: 10.2147/CIA.S149089

73. UpToDate. Antimicrobial agents that may induce Clostridium difficile diarrhea and colitis. In: . 2017. Accessed $12 / 232017$.

74. Davey P, Marwick C, Scott C, Charani E, McNeil K, Brown E, Gould I, Ramsay C, Michie S. Interventions to improve antibiotic prescribing practices for hospital inpatients. Cochrane Database Syst Rev. 2017; doi: 10.1002/14651858.CD003543.pub4

75. Dellit T, Owens R, McGowan J, Gerding D, Weinstein R, Burke J, Huskins WC, Paterson D, Fishman N, Carpenter 
C, Brennan PJ, Billeter M, Hooton T. Infectious Diseases Society of America and the Society for Healthcare Epidemiology of America guidelines for developing an institutional program to enhance antimicrobial stewardship. Clin Infect Dis. 2007; doi: 10.1086/510393.

76. Adam H, Baxter M, Davidson R, Rubinstein E, Fanella S, Karlowsky J, Lagacé-Wiens PRS, Hoban D, Zhanel G. Comparison of pathogens and their antimicrobial resistance patterns in paediatric, adult and elderly patients in Canadian hospitals. J Antimicrob Chemother. 2013; doi: 10.1093/jac/dkt024.

77. Mandell LA, Wunderink RG, Anzueto A, Bartlett JG, Campbell GD, Dean NC, Dowell SF, File TM,Jr, Musher DM, Niederman MS, Torres A, Whitney CG, Infectious Diseases Society of America, American Thoracic Society. Infectious Diseases Society of America/American Thoracic Society consensus guidelines on the management of community-acquired pneumonia in adults. Clin Infect Dis. 2007; doi: CID41620 [pii].

78. Prina E, Ranzani O, Torres A. Community-acquired pneumonia. Lancet. 2015; doi: 10.1016/S0140$6736(15) 60733-4$

79. Amalakuhan B, Echevarria KL, Restrepo MI. Managing community acquired pneumonia in the elderly - the next generation of pharmacotherapy on the horizon. Expert Opin Pharmacother. 2017; doi: 10.1080/14656566.2017.1340937

80. Dyar OJ, Huttner B, Schouten J, Pulcini C. What is antimicrobial stewardship?. Clin Microbiol Infect. 2017; doi: 10.1016/j.cmi.2017.08.026.

81. Dean NC, Silver MP, Bateman KA, James B, Hadlock CJ, Hale D. Decreased mortality after implementation of a treatment guideline for community-acquired pneumonia. Am J Med. 2001.

82. Dean N, Bateman K, Donnelly S, Silver M, Snow G, Hale D. Improved clinical outcomes with utilization of a community-acquired pneumonia guideline. Chest. 2006; doi: 10.1378/chest.130.3.794.

83. Fowler S, Webber A, Cooper BS, Phimister A, Price K, Carter Y, Kibbler CC, Simpson AJH, Stone SP. Successful use of feedback to improve antibiotic prescribing and reduce Clostridium difficile infection: a controlled interrupted time series. J Antimicrob Chemother. 2007; doi: 10.1093/jac/dkm014.

84. McNulty C, Logan M, Donald IP, Ennis D, Taylor D, Baldwin RN, Bannerjee M, Cartwright KA. Successful control of Clostridium difficile infection in an elderly care unit through use of a restrictive antibiotic policy. J Antimicrob Chemother. 1997.

85. Katz PR, Beam TR, Brand F, Boyce K. Antibiotic use in the nursing home. Physician practice patterns. Arch Intern Med. 1990

86. Katz M, Gurses A, Tamma P, Cosgrove S, Miller M, Jump RLP. Implementing Antimicrobial Stewardship in Long-term Care Settings: An Integrative Review Using a Human Factors Approach. Clin Infect Dis. 2017; doi: $10.1093 / \mathrm{cid} / \mathrm{cix} 566$.

87. Crnich C, Jump R, Trautner B, Sloane P, Mody L. Optimizing Antibiotic Stewardship in Nursing Homes: A Narrative Review and Recommendations for Improvement. Drugs Aging. 2015; doi: 10.1007/s40266-0150292-7.

88. Naughton BJ, Mylotte JM, Ramadan F, Karuza J, Priore RL. Antibiotic use, hospital admissions, and mortality before and after implementing guidelines for nursing home-acquired pneumonia. J Am Geriatr Soc. 2001.

89. Wenisch J, Equiluz Bruck S, Fudel M, Reiter I, Schmid A, Singer E, Chott A. Decreasing Clostridium difficile 
infections by an antimicrobial stewardship program that reduces moxifloxacin use. Antimicrob Agents Chemother. 2014; doi: 10.1128/AAC.03006-14.

90. Syrjälä H, Broas M, Suramo I, Ojala A, Lähde S. High-resolution computed tomography for the diagnosis of community-acquired pneumonia. Clin Infect Dis. 1998.

91. Hagaman J, Rouan G, Shipley R, Panos R. Admission chest radiograph lacks sensitivity in the diagnosis of community-acquired pneumonia. Am J Med Sci. 2009; doi: 10.1097/MAJ.0b013e31818ad805.

92. Wunderink R, Waterer G. Advances in the causes and management of community acquired pneumonia in adults. BMJ. 2017; doi: 10.1136/bmj.j2471.

93. Claessens Y, Debray M, Tubach F, Brun A, Rammaert B, Hausfater P, Naccache J, Ray P, Choquet C, Carette M, Mayaud C, Leport C, Duval X. Early Chest Computed Tomography Scan to Assist Diagnosis and Guide Treatment Decision for Suspected Community-acquired Pneumonia. Am J Respir Crit Care Med. 2015; doi: 10.1164/rccm.201501-00170C.

94. Llamas Álvarez A, Tenza Lozano E, Latour Pérez J. Accuracy of Lung Ultrasonography in the Diagnosis of Pneumonia in Adults: Systematic Review and Meta-Analysis. Chest. 2017; doi: 10.1016/j.chest.2016.10.039.

95. Oosterheert J, van Loon A, Schuurman R, Hoepelman AIM, Hak E, Thijsen S, Nossent G, Schneider MME, Hustinx WMN, Bonten MJM. Impact of rapid detection of viral and atypical bacterial pathogens by real-time polymerase chain reaction for patients with lower respiratory tract infection. Clin Infect Dis. 2005; doi: $10.1086 / 497134$.

96. Hernes SS, Hagen E, Quarsten H, Bjorvatn B, Bakke PS. No impact of early real-time PCR screening for respiratory viruses on length of stay and use of antibiotics in elderly patients hospitalized with symptoms of a respiratory tract infection in a single center in Norway. Eur J Clin Microbiol Infect Dis. 2014; doi: 10.1007/ s10096-013-1963-0.

97. Tobia C, Aspinall S, Good C, Fine M, Hanlon J. Appropriateness of antibiotic prescribing in veterans with community-acquired pneumonia, sinusitis, or acute exacerbations of chronic bronchitis: a cross-sectional study. Clin Ther. 2008; doi: 10.1016/j.clinthera.2008.06.009. 
abo

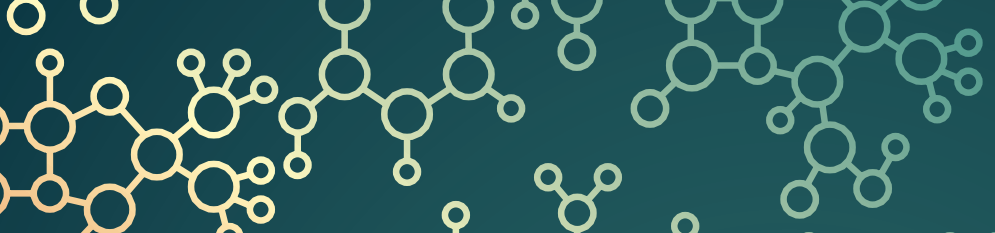
o

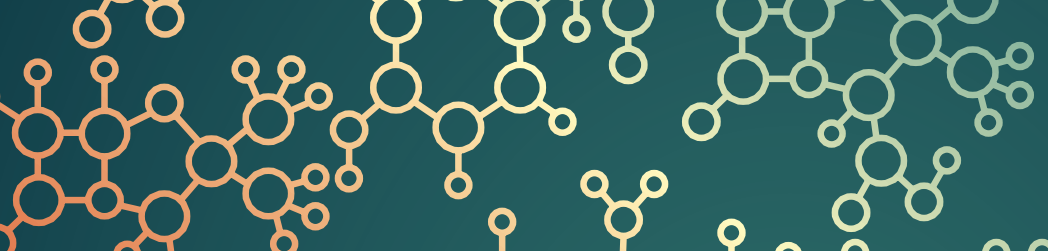

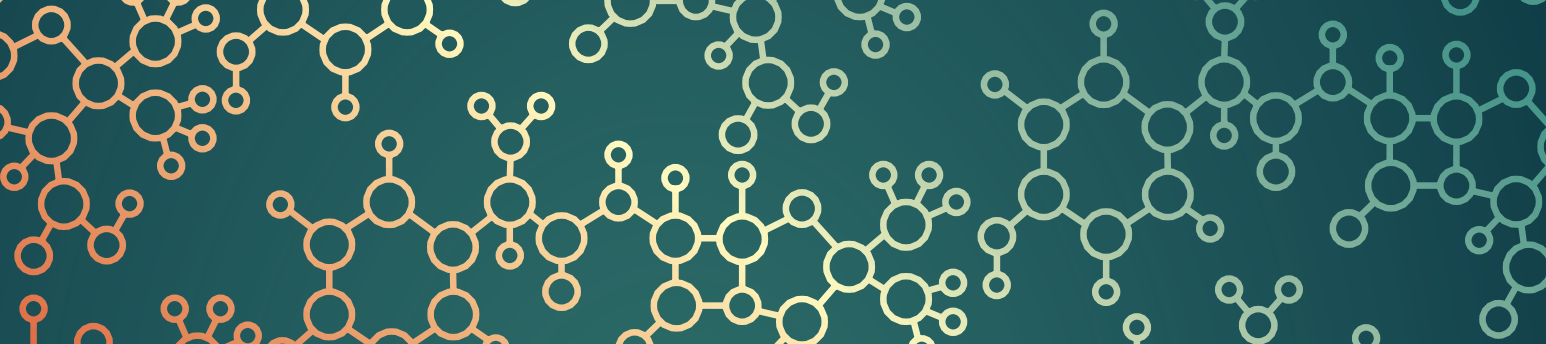

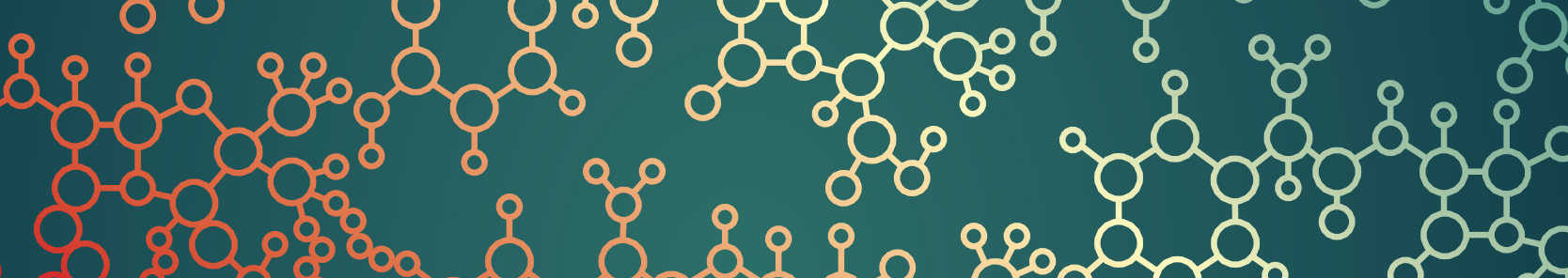
80\%

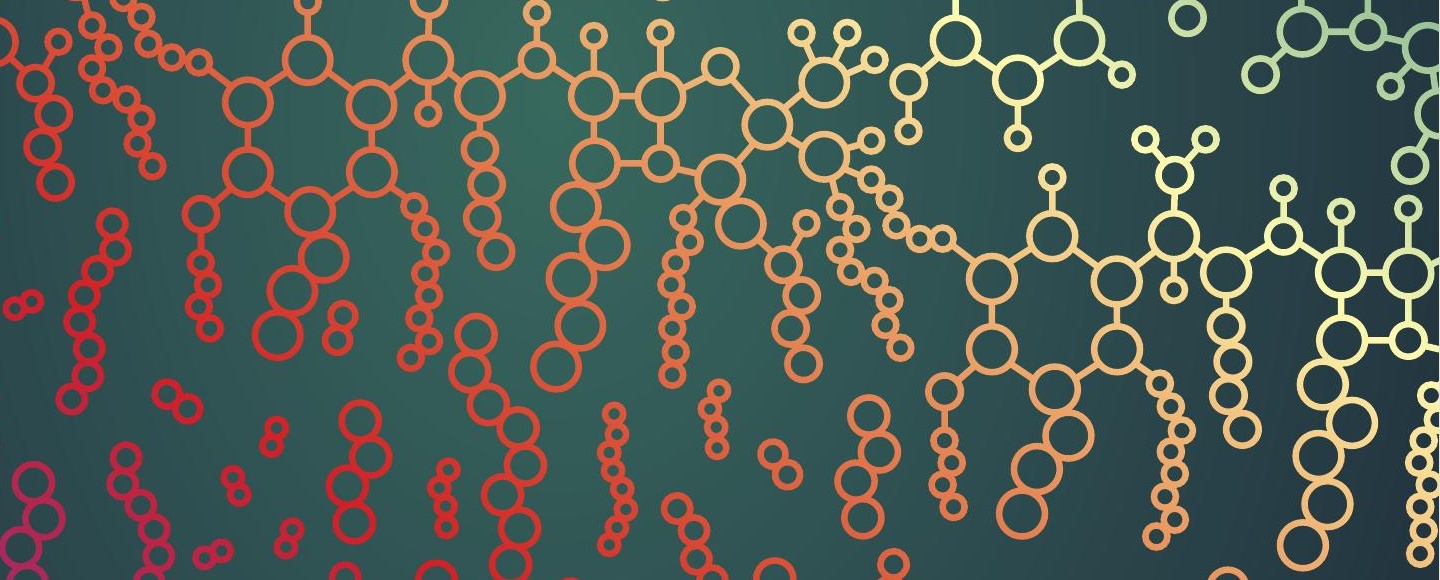
$88 \% 888$ $\circ 88$ \& 8

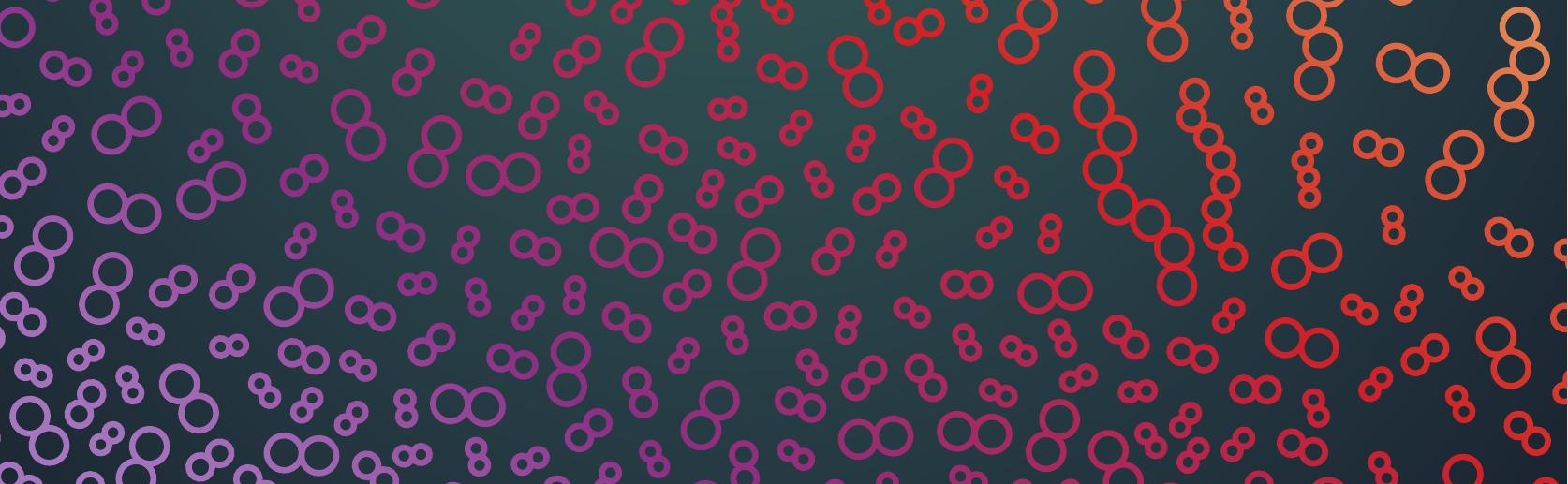


Narrow-spectrum

antibiotics for community-

acquired pneumonia in adults:

a stepped-wedge cluster randomized trial.

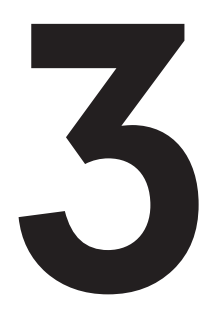

Inger van Heijl*, Valentijn A. Schweitzer*, Jan Jelrik Oosterheert, Wendelien DorigoZetsma, Paul D. van der Linden, C.H. Edwin Boel, Cornelis H. van Werkhoven, Marc J.M. Bonten, on behalf of CAP-PACT study group.

*These authors contributed equally to this manuscript 


\section{ABSTRACT}

\section{Background}

Adults hospitalized with moderately-severe community-acquired pneumonia (CAP) are often treated with broad-spectrum antibiotics, despite guidelines recommending narrow-spectrum antibiotics. We determined safety and effectiveness of a multifaceted antibiotic stewardship intervention to increase the use of narrow-spectrum antibiotics.

\section{Methods}

In a stepped-wedge cluster-randomized trial in twelve hospitals we implemented an intervention consisting of education, motivating opinion leaders, and audit and feedback and determined its effect on the days of therapy (DOT) with narrow-spectrum antibiotics (penicillin, amoxicillin or doxycycline) and all-cause 90-day mortality. In an intention-totreat analysis we used 90-day all-cause mortality as co-primary safety outcome (noninferiority margin 3\% and one-sided alpha of 0.05 ) and reduction in DOT with narrowspectrum antibiotics as co-primary effectiveness outcome.

\section{Results}

From November 2015 till November 2017 4,084 patients were included; 2,235 in the control and 1,849 in the intervention period. Median age was 73 (range 18 - 101) years, $53.0 \%$ (2163/4084) were male and mean Pneumonia Severity Index score was 91.3 (SD \pm 31.4 ). The adjusted relative reduction in broad-spectrum DOT during intervention was $26.9 \%(95 \% \mathrm{Cl}: 15.4 \%-37.4 \%)$ with averages of 6.6 and 4.8 days in the control and intervention period, respectively. Crude 90-day mortality was $10.9 \%$ (242/2228) and $10.8 \%(199 / 1841)$ during control and intervention period, yielding an adjusted absolute difference of $0.4 \%(90 \% \mathrm{Cl}:-2.7$ to 2.4$)$ for the control versus intervention period, indicating non-inferiority for all-cause mortality.

\section{Conclusions}

In patients hospitalized with moderately-severe CAP a multifaceted antibiotic stewardship intervention safely reduced the days of broad-spectrum antibiotic use with $27 \%$. (CAPPACT ClinicalTrials.gov number, NCT02604628.) 


\section{INTRODUCTION}

Community-acquired pneumonia (CAP) is associated with high morbidity and mortality ${ }^{[1,2]}$. International guidelines recommend to base empiric antibiotic treatment of patients with CAP on the severity of disease. Accordingly, CAP may be classified as moderatelysevere based on the need of hospital admission in a non-intensive care unit (ICU) ward (pragmatic classification); on a pneumonia severity index (PSI) score of 3-4; or on a CURB-65 score of $2{ }^{[3]}$. Recommended empirical antibiotic treatments of moderatesevere CAP differ and include narrow-spectrum beta-lactam monotherapy (such as penicillin $G$ or amoxicillin) ${ }^{[4,5]}$, beta-lactam macrolide combination therapy ${ }^{[6,7]}$ or fluoroquinolone monotherapy ${ }^{[7]}$. Empiric use of narrow-spectrum beta-lactams exerts less selective pressure for antibiotic resistance, compared to more broad-spectrum antibiotics. However, high quality evidence for equal clinical effectiveness of narrowspectrum and more broad-spectrum beta-lactam monotherapy is limited to two randomized trials comparing amoxicillin monotherapy to fluoroquinolones. One study used a fluoroquinolone no longer in use (sparfloxacin) and the other study only included patients with mild pneumonia ${ }^{[10,11]}$. Adherence to guideline recommendations for using narrow-spectrum beta-lactams as empiric treatment in patients with moderatesevere CAP is low ${ }^{[12]}$. Antimicrobial stewardship may improve guideline adherence and appropriateness of empiric antibiotic therapy ${ }^{[13]}$. Furthermore, high-quality evidence on safety and effectiveness of narrow-spectrum beta-lactam monotherapy in patients with moderate-severe CAP could augment confidence in adaptation of such treatment strategies.

We, therefore, investigated whether implementation of a multifaceted antimicrobial stewardship intervention reduced broad-spectrum antibiotic use without compromising patients safety.

\section{METHODS}

The "Community-Acquired Pneumonia increasing Protocol adherence by Antibiotic stewardship in a stepped-wedge Cluster-randomized Trial" (CAP-PACT) trial was an investigator-initiated stepped wedge cluster randomized quality improvement trial. The study was reviewed by the ethics review board of the University Medical Centre Utrecht (reference number 15/100), and local antimicrobial stewardship teams consented to participate in the study. As the implemented stewardship intervention promoted bestpractice antibiotic therapy as described in the guidelines, as the intervention was aimed 
at healthcare providers rather than individual patients, and as anonymised routinely collected data were used, the need to obtain individual informed consent was waived. Data are reported according to the CONSORT guidelines of stepped wedge cluster randomised trials and non-inferiority trials ${ }^{[14]}$. The trial was prospectively registered at clinicaltrials.gov (identifier NCT02604628).

\section{Patients and study setting}

The trial was performed from November 2015 till November 2017 in 12 hospitals in the Netherlands. All Dutch hospitals were eligible for study participation. Participating hospitals were university hospitals $(n=2)$, teaching hospitals $(n=7)$, and non-teaching hospitals $(n=3)$. Consecutive adult patients of 18 years or older receiving antibiotic therapy for a working diagnosis of CAP and admitted to a non-ICU ward were enrolled. Patients were not eligible if they: recently ( $\leq 14$ days) resided in a nursing home or long-term care facility; were recently ( $\leq 14$ days) admitted to an acute care hospital for two or more days; were known to have cystic fibrosis; or were immunocompromised. Immunocompromised was defined as: having a human immunodeficiency virus infection with a last CD4 cell count of $<300$ cells $/ \mu \mathrm{L}$; having received cytotoxic chemotherapy or radiotherapy in the previous 3 months; being on chronic (>3 months) haemodialysis; having received a solid organ or bone marrow transplant; or receiving immunosuppressive therapy. In the Netherlands every hospital has a specialised antimicrobial stewardship team responsible for implementing antimicrobial stewardship interventions, consisting of at least a clinical microbiologist, an infectious disease specialist and a hospital pharmacist. Three out of twelve hospitals stopped participation before they entered the intervention period, either because the principal investigator stopped research activities $(n=2)$ or because they were no longer able to collect study data $(n=1)$. Data from these hospitals was not used for analysis.

\section{Antimicrobial stewardship intervention}

The intervention was a multifaceted bundle based on proven effective stewardship interventions ${ }^{[15]}$. The bundle consisted of (1) education, (2) activating local opinion leaders, and (3) prospective audit and feedback of antibiotic use. Educational activities were targeted at physicians of pulmonary and internal medicine departments and consisted of clinical lessons, e-learning and educational attributes. Clinical lessons were given in regular intervals of 6 months in which the CAP guidelines were addressed using case-based discussions with feedback of antibiotic prescribing data of the respective hospital, which were anonymously benchmarked against other participating hospitals. At the start of the intervention period physicians of the participating hospitals were invited to complete the e-learning, containing case-based questions about the CAP guideline. Invites to 
complete the e-learning were send periodically to reach new employees. In addition, educational attributes in the form of posters and pocket cards summarizing the CAP guidelines were distributed at the start of the intervention. At intervention introduction, local opinion leaders were identified in collaboration with the local antibiotic stewardship team, and actively involved in the study and stewardship activities. They were asked to stimulate guideline adherent treatment throughout the intervention period, e.g. during hand-over meetings. Thirdly, prospective audit and feedback was implemented during the intervention period by the local antimicrobial stewardship team. On week days all patients admitted with moderate-severe CAP were actively identified and responsible physicians were contacted by a member of the local antimicrobial stewardship team to switch treatment to penicillin or amoxicillin monotherapy if treatment was not according to the guideline recommendation. If, for any reason, treatment could not be switched it was recommended to perform a pneumococcal urine antigen test to facilitate deescalation if the test result was positive. Recommendations were done by telephone and were registered in electronic health records, as were reasons for not accepting recommendations.

\section{Outcomes}

The study had two co-primary outcomes: broad-spectrum days of therapy (DOT) per patient and all-cause 90-day mortality. Days that patients received antibiotic treatment were classified as narrow-spectrum DOT if amoxicillin, penicillin or doxycycline monotherapy was given and as broad-spectrum DOT if any other antibiotic regimen was administered. Doxycycline monotherapy was defined as narrow-spectrum therapy as it is recommended in our national guideline as equivalent to amoxicillin for mild CAP. Vital status at day 90 was derived from the municipal records database if not evident from the medical records. Secondary outcomes were narrow-spectrum DOT, total DOT, 30-day mortality, length of hospital stay, hospital readmissions within 30-days of hospital admission, intensive care unit admissions, complications, Clostridioides difficile associated disease and antibiotic switches.

\section{Randomisation}

The unit of randomization was the hospital and comprised the sequence in which they would crossover from control to intervention period (Figure S1). Randomisation was performed electronically by a data manager blinded to the intervention after recruitment of all hospitals. All the participating hospitals started with a control period and every 3 months a block of two hospitals transitioned from control to intervention, with all hospitals eventually ending in the intervention period. Allocation to the time of intervention implementation was concealed for treating physicians. 


\section{Statistical analysis}

The detailed statistical analysis plan was published at clinicaltrials.gov (NCT02604628) before database lock. The sample size calculation was determined by non-inferiority for 90-day mortality. Assuming a 90-day mortality of $10 \%$, a non-inferiority margin of $3 \%$, and a one-sided alpha 0.05 , a total of 4464 patients were required for $80 \%$ power. Primary intention-to-treat (ITT) analyses were performed using mixed effect models including random intercept and fixed slope per hospital, time as fixed effect, adjusted for the following potential confounders as fixed effects: PSI-score, smoking status, COPD, diabetes mellitus, and antibiotic pre-treatment. For 90-day mortality we used a mixed effects logistic regression, recalculated to risk differences ${ }^{[16]}$. As secondary analyses we performed as-treated analysis and a Complier Average Causal Effect (CACE) analysis, both aiming to estimate the difference in mortality between patients empirically treated with narrow-spectrum versus broad-spectrum antibiotics. The CACE was used with randomisation as an instrumental variable to estimate the ITT adjusted for noncompliance ${ }^{[17]}$. DOTs were analysed using mixed effects negative binomial models with robust standard errors. Length of hospital stay was analysed using mixed effects Cox proportional hazards models using in-hospital mortality as a competing event, assuming maximum follow-up duration when censored. ICU admissions, hospital readmissions and antibiotic switches were analysed using mixed effects logistic regression. Missing data were imputed by multiple imputation, with the exception of data on respiratory rate, heart rate, and confusion at admission which were assumed to be normal when not documented in the medical charts. No transition period was used because the treatment effect of the audit and feedback was assumed to be immediate. All analyses were performed using R statistical software version 3.5.1.

\section{RESULTS}

A total of 4084 patients with CAP admitted to a non-ICU ward were included during the study period; 2,235 during the control period and 1,849 during the intervention period (Figure 1). The baseline characteristics of patients in the control period were similar to patients in the intervention period (Table 1). The most commonly identified pathogen was Streptococcus pneumoniae 488 (11.9\%), followed by Haemophilus influenza 285 (7.0\%), and Staphylococcus aureus 97 (2.4\%), with no differences between the control and intervention period (Table S1). 


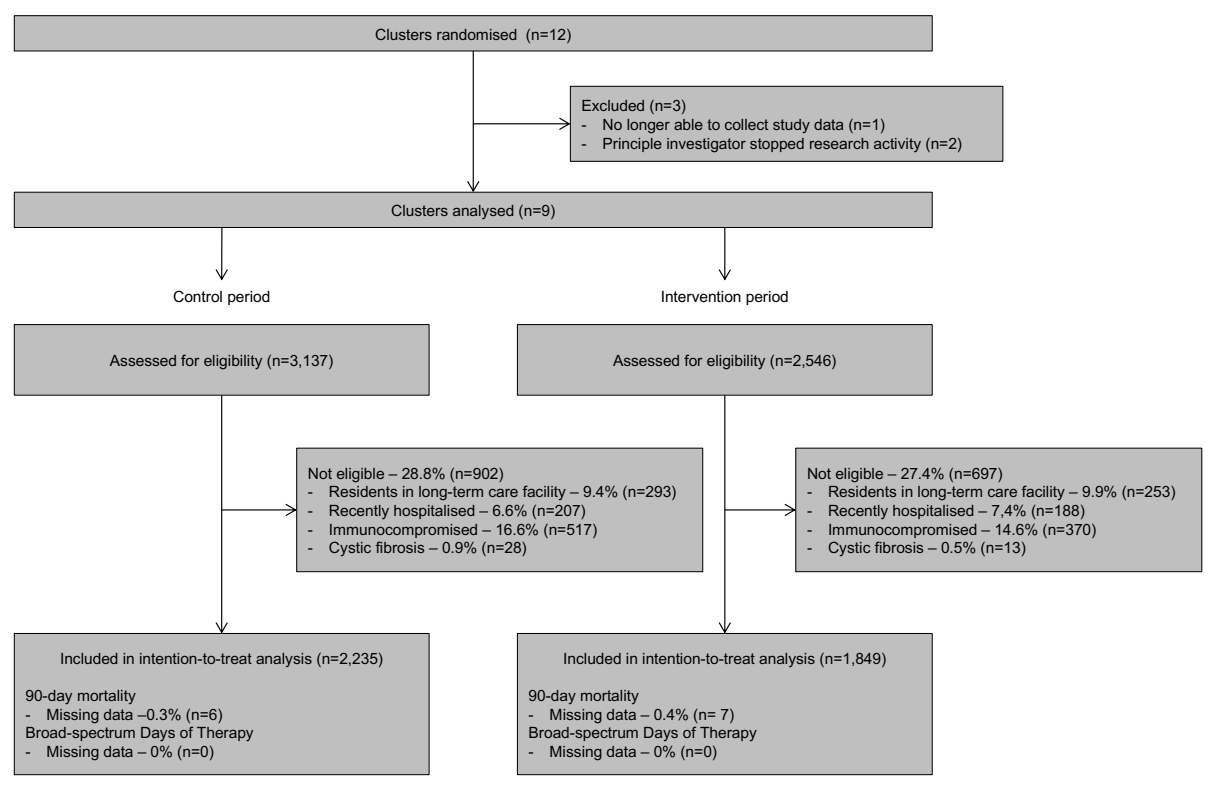

Figure 1 Flowchart of patient inclusion during the study period.

\section{Antimicrobial stewardship intervention implementation}

In the intervention periods, a total of 54 clinical lessons were given, with an average interval of 90.0 days ( 3 months) and 235 e-learnings were completed (Table S2). The local stewardship teams offered 330 recommendations for changing antibiotics, of which 197 (59.7\%) were accepted. Most common reasons for not accepting recommendations were patients having COPD $(11,8.3 \%)$, and patients with severe pneumonia according to PSI or CURB score or admitted with clinical deterioration (9; 6.8\%).

\section{Primary outcomes}

The median broad-spectrum DOT/patient was 6 (interquartile range (IQR) 2-9) in the control and 3 (IQR 0-8) in the intervention period, yielding an adjusted relative reduction in broad-spectrum DOTs of $26.9 \%$ (95\% confidence interval (CI) 15.4\%-37.4\%) (Table S3). Adjusted relative reductions in broad-spectrum DOT per hospital ranged from $16.7 \%$ to 39.3\% (Table S4). The median narrow-spectrum DOT/patient were 0 (IQR 0-6) and 5 (IQR 0-8), while the median total DOT/patient were 8 (IQR 7-10) in the control and 8 (IQR 7-11) in the intervention period (Figure 2). Most common prescribed empiric antibiotic regimens in the control and intervention period respectively were narrow-spectrum antibiotics (28.5\% vs. $45.1 \%$ ), broad-spectrum beta-lactam monotherapy $133.8 \%$ vs. $26.9 \%$ ), and beta-lactam fluoroquinolone combination therapy (27.3\% vs. $23.2 \%$ ) (Table 
S5). Overall narrow-spectrum antibiotic use mostly consisted of amoxicillin (1303/1409, 92.5\%), followed by penicillin (48/1409, 3.4\%), and doxycycline (139/1409, 9.9\%).

Table 1. Baseline characteristics of patients in the control period compared to patients in the intervention period

\begin{tabular}{|c|c|c|}
\hline & Control $(n=2,235)$ & Intervention $(n=1,849)$ \\
\hline Age (years, median, IQR) & $73(63-81)$ & $74(64-82)$ \\
\hline Male gender $(n, \%)$ & $1,188(53.2)$ & $975(52.7)$ \\
\hline Antibiotic use before admission ( $n, \%)$ & $742(33.2)$ & $569(30.8)$ \\
\hline \multicolumn{3}{|l|}{ Smoke behaviour $(n, \%)$} \\
\hline Active smoker & $512(27.2)$ & $412(27.6)$ \\
\hline Past smoker & $725(38.5)$ & $626(41.9)$ \\
\hline Never smoked & $396(21.1)$ & $264(17.7)$ \\
\hline \multicolumn{3}{|l|}{ Medical speciality $(n, \%)$} \\
\hline Internal medicine & $416(18.6)$ & $349(18.9)$ \\
\hline Pulmonology & $1731(77.4)$ & $1426(77.1)$ \\
\hline \multicolumn{3}{|l|}{ Co-morbid conditions ( $\mathrm{n}, \%)$} \\
\hline COPD or asthma & $962(43.0)$ & $880(47.6)$ \\
\hline Cardiovascular disease & $300(13.4)$ & $259(14.0)$ \\
\hline Diabetes mellitus & $389(17.4)$ & $315(17.0)$ \\
\hline Malignancy & $239(10.7)$ & $185(10.0)$ \\
\hline PSI score (median, IQR) & $89(70-112)$ & $91(72-113)$ \\
\hline PSI risk class I (n, \%) & $101(4.5)$ & $73(3.9)$ \\
\hline PSI risk class II $(n, \%)$ & $473(21.2)$ & $357(19.3)$ \\
\hline PSI risk class III $(n, \%)$ & $581(26.0)$ & $493(26.7)$ \\
\hline PSI risk class IV $(n, \%)$ & $823(36.8)$ & $722(39.0)$ \\
\hline PSI risk class $\vee(n, \%)$ & $257(11.5)$ & $204(11.0)$ \\
\hline CURB-65 score (median, IQR) & $2(1-2)$ & $2(1-2)$ \\
\hline Had radiologically confirmed CAP $(n, \%)$ & $1689(75.6)$ & $1377(74.5)$ \\
\hline Blood culture obtained (n, \%) & $1602(71.7)$ & $1387(75.0)$ \\
\hline Sputum culture obtained (n, \%) & $888(39.7)$ & $784(42.4)$ \\
\hline PUAT performed (n, \%) & $965(43.2)$ & $1173(63.4)$ \\
\hline LUAT performed $(n, \%)$ & $1297(58.0)$ & 1255 (67.9) \\
\hline
\end{tabular}

COPD chronic obstructive pulmonary disease, PUAT pneumococcal urinary antigen test, LUAT legionella urinary antigen test, PSI pneumonia severity index, IQR interquartile range, CURB- 65 pneumonia severity score based on presence of confusion, blood ureum levels, respiratory rate, blood pressure, and age, CAP community-acquired pneumonia

All-cause 90 -day mortality was $10.9 \%(242 / 2228)$ in the control period and $10.8 \%$ $(199 / 1841)$ in the intervention period (Figure S2). In the ITT analysis, the adjusted risk difference in 90 -day mortality was $0.4 \%(90 \% \mathrm{Cl}-2.7 \%$ to $2.4 \%)$. As the confidence interval does not contain the pre-specified non-inferiority margin of $3 \%$ we conclude that the intervention is non-inferior to the control period (Figure 3). Sensitivity analyses for patients with radiologically confirmed CAP yielded similar results. Results were comparable in the as-treated and CACE analysis (Table S6). 


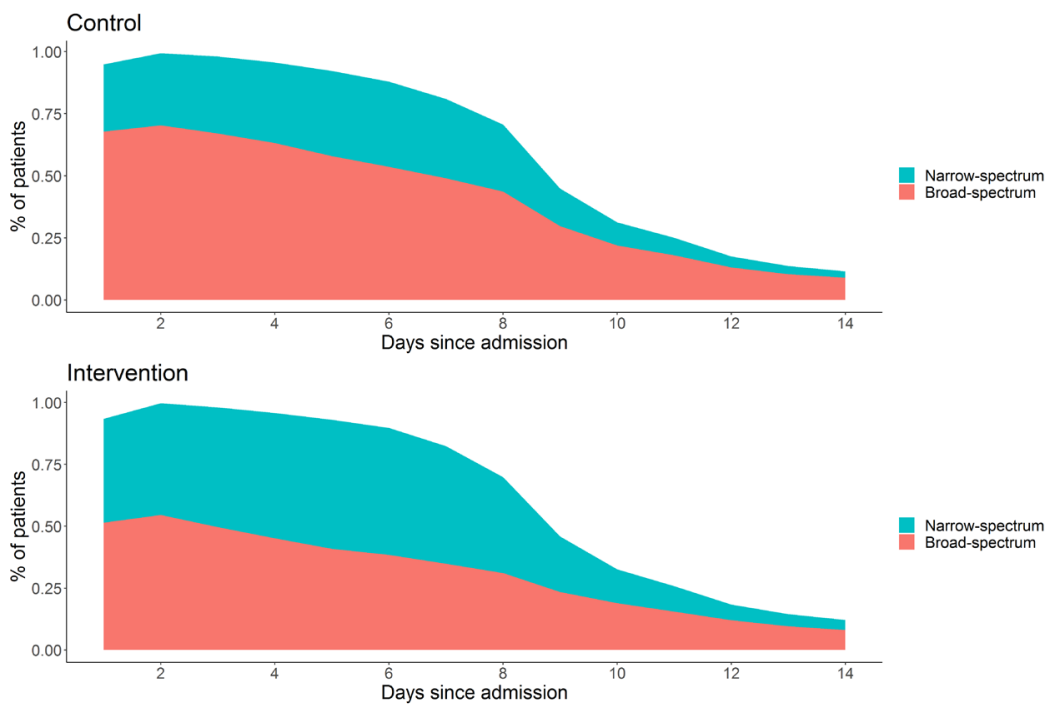

Figure 2 Proportion of patient receiving narrow-spectrum antibiotics (green) or broad-spectrum antibiotics (red) in days since hospital admission.

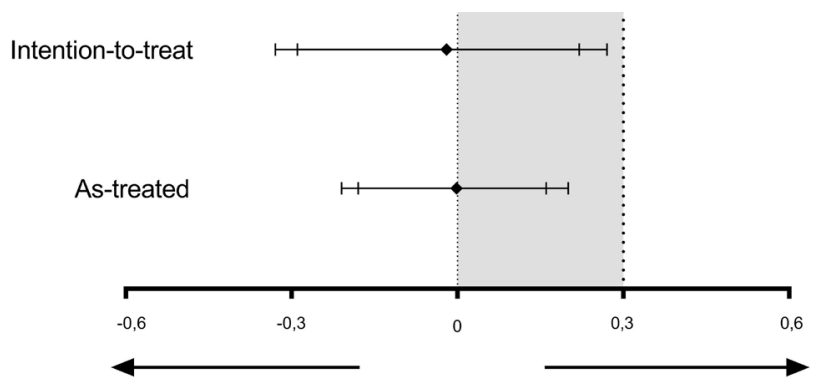

Mortality in control period higher

Mortality in intervention period higher

Figure 3 Non-inferiority plots

\section{Secondary outcomes}

All-cause 30-day mortality was $6.9 \%(n=154)$ in the control and $6.7 \%(n=123)$ in the intervention period (Table 2). Results were comparable in the as-treated and CACE analysis (Table S7). Median length of hospital stay was 5 (IQR 3-8) days in the control and 5 (IQR 3-8) days in the intervention period. Comparable rates were observed for ICU-admissions (94, 4.2\% control vs 38, 2.1\% intervention), hospital readmissions (243, $11.3 \%$ control vs $203,11.4 \%$ intervention), complications $(380,17.0 \%$ control vs 332 , 18.0\% intervention) (Table S8), Clostridioides difficile associated disease (2, $0.1 \%$ control 
vs $6,0.3 \%$ intervention), and i.v. to oral switches $(1469,80.8 \%$ control vs $1262.83 .8 \%$ intervention). Switches from broad to narrow-spectrum occurred in $26.4 \%(n=421)$ in the control and $41.3 \%(n=413)$ in the intervention period.

Table 2 Secondary outcomes

\begin{tabular}{|c|c|c|c|c|}
\hline & $\begin{array}{l}\text { Control } \\
(n=2,240)\end{array}$ & $\begin{array}{l}\text { Intervention } \\
(n=1,844)\end{array}$ & $\begin{array}{l}\text { Crude estimates } \\
(95 \% \mathrm{Cl})\end{array}$ & $\begin{array}{l}\text { Adjusted estimates } \\
(95 \% \mathrm{Cl})\end{array}$ \\
\hline 30-day mortality (n, \%) & $154(6.9 \%)$ & $123(6.7 \%)$ & $\mathrm{RD}-0.3(-1.6-1.1)$ & $\mathrm{RD}-1.1(-3.1-0.7)$ \\
\hline Intensive care unit admissions ( $\mathrm{n}, \%$ ) & $94(4.2 \%)$ & $38(2.1 \%)$ & OR $0.5(0.3-0.7)$ & OR $0.3(0.2-0.6)$ \\
\hline Hospital readmissions (n, \%) & $243(11.3 \%)$ & $203(11.4 \%)$ & OR $1.0(0.8-1.2)$ & OR $1.2(0.8-1.7)$ \\
\hline Switch from NS to BS ( $n, \%)$ & $148(23.0 \%)$ & $195(23.0 \%)$ & OR $1.0(0.8-1.3)$ & OR $1.0(0.7-1.5)$ \\
\hline Switch from i.v. to oral (n, \%) & $1469(80.8 \%)$ & $1262(83.8 \%)$ & OR $1.2(1.0-1.5)$ & OR $1.3(0.9-1.8)$ \\
\hline Switch from oral to i.v. (n, \%) & $44(10.6 \%)$ & $26(7.6 \%)$ & OR $0.7(0.4-1.2)$ & OR $0.7(0.3-1.8)$ \\
\hline
\end{tabular}

BS broad-spectrum antibiotics, NS narrow-spectrum antibiotics, i.v. intravenous, RD risk difference, HR hazard ratio, OR odds ratio

\section{DISCUSSION}

In this stepped wedge cluster randomised trial a multifaceted antimicrobial stewardship intervention based on education and audit and feedback resulted in a $27 \%$ reduction in broad-spectrum antibiotic use in patients hospitalized with moderate-severe CAP without compromising patient outcome at day 90 after hospital admission.

The stewardship intervention bundle contained elements that are considered effective in optimizing antibiotic use ${ }^{[13,15]}$. In meta-analysis the risk difference for guideline adherent prescription after implementation of antimicrobial stewardship interventions was 15\% (95\% Cl 14\%-16\%) $\left.{ }^{[13]}\right)$. Compared to this, our bundle was more effective. However, heterogeneity was large between hospitals, with reductions ranging from $21 \%$ to $37 \%$. Heterogeneity might have resulted from different barriers (reasons) for not prescribing narrow-spectrum antibiotics between hospitals. Therefore, even though the components of our bundle are evidence based, it might be a more optimal use of resources to first identify barriers for non-adherent prescribing and tailor the intervention to the specific setting based on behaviour change theory ${ }^{[18]}$.

The optimal empiric treatment for moderate-severe CAP is still subject to debate. Thus far, two high quality RCTs have investigated the effects of empirical coverage for atypical pathogens on patient outcome ${ }^{[8,19]}$. In a multicentre non-inferiority RCT beta-lactam 
monotherapy was not non-inferior to beta-lactam macrolide combination therapy for the time till reaching clinical stability. Patients randomized to beta-lactam monotherapy had a $7.6 \%$ higher absolute risk for not being clinically stable at day 7 . The one-sided $90 \%$ $\mathrm{Cl}$ was $13 \%$ which crossed the pre-defined non-inferiority margin of $8 \%$. In a multicentre cluster randomised cross-over trial a strategy of preferred empiric treatment with beta-lactam monotherapy was compared to strategies with beta-lactam macrolide combination therapy and fluoroquinolone monotherapy. In this study, the beta-lactam monotherapy strategy was non-inferior to the broader regimens regarding 90-day mortality ( 2 sided $90 \% \mathrm{Cl}$ not overlapping the non-inferiority margin of $3 \%$ ).

The equipoise is also reflected in international guideline recommendations for empiric treatment of moderate-severe CAP. American and British guidelines recommend betalactam macrolide combination therapy or fluoroquinolone monotherapy ${ }^{[6,7]}$ while Swedish, Danish and Dutch guidelines recommend narrow-spectrum beta-lactam monotherapy ${ }^{[3-}$ ${ }^{5]}$. The rationale for empiric treatment with narrow-spectrum beta-lactam monotherapy is, first, that the most common causative pathogen in CAP, Streptococcus pneumoniae, is susceptible to these antibiotics, and second that the severity of disease allows escalation within 48 hours to broader antibiotic therapy based on diagnostic testing or lack of clinical improvement. Thus far, two RCTs have evaluated the efficacy of narrow-spectrum betalactams in moderate-severe CAP patients ${ }^{[10,11]}$. Petitpretz et al. compared moxifloxacin to amoxicillin in patients with mild-to-moderate suspected pneumococcal CAP and clinical success rates were $86.5 \%(173 / 200)$ and $82.2 \%(171 / 208)$ in the moxifloxacin and amoxicillin treated patients, respectively. In the other RCT clinical cure rates were $83.6 \%$ $(133 / 159)$ and $84.7 \%(144 / 170)$ in adult patients with community-acquired suspected pneumococcal pneumonia treated with sparfloxacin and amoxicillin, respectively. Yet, in clinical practice physicians apparently are reluctant to use narrow-spectrum betalactams, as demonstrated by the low adherence to current guideline recommendations in the control period of this study. Our findings provide further evidence that more patients with moderate-severe CAP can be safely empirically treated with narrowspectrum antibiotics, which contributes to more prudent use of antibiotics.

Our study has several limitations. First, because we implemented a multifaceted bundle of multiple stewardship interventions it is not possible to estimate the effect of the individual components. However, the approach reflects clinical practice, where stewardship interventions are usually implemented as bundles. Second, the nature of the intervention precluded a blinded evaluation, and, therefore, information bias cannot be excluded. To minimize the impact of information bias we chose objective primary outcomes and used trained research nurses and standardized methodology for data collection. Third, in 
cluster randomized stepped wedge designs selection bias may occur if different patients are included in both study periods. Yet, baseline characteristics of patients in the control versus intervention period were similar and we adjusted for important prognostic factors. The cluster randomized design has major advantages because it reduces contamination of the intervention and better reflects clinical practice where a stewardship bundle gets implemented, than individual patient randomization. As a result, the study design as used has high generalizability. Fourth, the inclusion of patients without radiologically confirmed CAP may have diluted the effect to non-inferiority for mortality. However, as the study focusses on effectiveness rather than efficacy, the chosen study population (i.e., those being treated for presumed CAP) closely represents clinical practice. In addition, studies using low-dose CT scanning in addition to chest $X$-ray have demonstrated that radiological infiltrates are not apparent on chest radiographs in approximately $30 \%$ of patients with CAP based on CT findings ${ }^{[20]}$. This implies that many patients with CAP would be excluded if enrolment was based on presence of chest X-ray findings. Moreover, in the current study similar results were obtained in the subset of patients with radiologically confirmed CAP. Fifth, the study was performed in a setting with low prevalence of antimicrobial resistance and atypical pathogens, which may limit generalisability to settings with more resistance and atypical pathogens. However, Streptococcus pneumoniae with reduced susceptibility to penicillin can still be successfully treated with high dose penicillin's ${ }^{[21]}$.

To conclude, a multifaceted antimicrobial stewardship intervention focused on education and audit and feedback reduced broad-spectrum antibiotic use by $27 \%$ in immunocompetent patients hospitalized with moderate-severe CAP and was noninferior in terms of all-cause 90-day mortality. These results indicate that more patients with moderate-severe CAP can be safely treated with narrow-spectrum antibiotics.

\section{List of abbreviations}

BS, broad-spectrum antibiotics; CACE, Complier Average Causal Effect; CAP, Communityacquired pneumonia; CAP-PACT, Community-Acquired Pneumonia increasing Protocol adherence by Antibiotic stewardship in a stepped-wedge Cluster-randomized Trial; DOT, days of therapy; HR, hazard ratio; ICU, intensive care unit; IQT, interquartile range; ITT, intention-to-treat; i.v., intravenous; NS, narrow-spectrum antibiotics; OR, odds ratio; $\mathrm{PSI}$, pneumonia severity index; RD, risk difference.

\section{Acknowledgements}

None. 


\section{Funding}

This research received no specific grant from any funding agency in the public, commercial, or not-for-profit sectors.

\section{Conflict of interests}

No conflict of interest to disclose. 


\section{REFERENCES}

1. Prina E, Ranzani OT, Torres A. Community-acquired pneumonia. Lancet (London, England), 386(9998), 10971108 (2015).

2. Wunderink RG, Waterer GW. Clinical practice. Community-acquired pneumonia. N Engl J Med, 370(6), $543-$ 551 (2014)

3. Wiersinga WJ, Bonten MJ, Boersma WG et al. Management of community-acquired pneumonia in adults: 2016 guideline update from the Dutch Working Party on Antibiotic Policy (SWAB) and Dutch Association of Chest Physicians (NVALT). Neth J Med, 76(1), 4-13 (2018).

4. Athlin S, Lidman C, Lundqvist A et al. Management of community-acquired pneumonia in immunocompetent adults: updated Swedish guidelines 2017. Infect Dis (Lond), 50(4), 247-272 (2018)

5. Fally MM-W, U.; Lynghøj-Nielsen, T.; Stæhr Jensen, JU. Pneumoni - initial undersøgelse og behandling. (2017). https://www.lungemedicin.dk/fagligt/71-pneumoni.html

6. Lim WS, Baudouin SV, George RC et al. BTS guidelines for the management of community acquired pneumonia in adults: update 2009. Thorax, 64 Suppl 3, iii1-55 (2009)

7. Metlay JP, Waterer GW, Long AC et al. Diagnosis and Treatment of Adults with Community-acquired Pneumonia. An Official Clinical Practice Guideline of the American Thoracic Society and Infectious Diseases Society of America. Am J Respir Crit Care Med, 200(7), e45-e67 (2019).

8. Postma DF, van Werkhoven $\mathrm{CH}$, van Elden LJ et al. Antibiotic treatment strategies for community-acquired pneumonia in adults. N Engl J Med, 372(14), 1312-1323 (2015)

9. Garau J. Treatment of drug-resistant pneumococcal pneumonia. The Lancet. Infectious diseases, 2(7), 404415 (2002).

10. Aubier M, Verster R, Regamey C, Geslin P, Vercken JB. Once-daily sparfloxacin versus high-dosage amoxicillin in the treatment of community-acquired, suspected pneumococcal pneumonia in adults. Sparfloxacin European Study Group. Clin Infect Dis, 26(6), 1312-1320 (1998).

11. Petitpretz P, Arvis P, Marel M, Moita J, Urueta J. Oral moxifloxacin vs high-dosage amoxicillin in the treatment of mild-to-moderate, community-acquired, suspected pneumococcal pneumonia in adults. Chest, 119(1), 185-195 (2001)

12. Huijts SM, van Werkhoven CH, Boersma WG et al. Guideline adherence for empirical treatment of pneumonia and patient outcome. Treating pneumonia in the Netherlands. Neth J Med, 71(10), 502-507 (2013).

13. Davey P, Marwick CA, Scott CL et al. Interventions to improve antibiotic prescribing practices for hospital inpatients. Cochrane Database Syst Rev, 2, Cd003543 (2017).

14. Hemming K, Taljaard M, McKenzie JE et al. Reporting of stepped wedge cluster randomised trials: extension of the CONSORT 2010 statement with explanation and elaboration. BMJ (Clinical research ed.), 363, k1614 (2018)

15. Dellit TH, Owens RC, McGowan JE, Jr. et al. Infectious Diseases Society of America and the Society for Healthcare Epidemiology of America guidelines for developing an institutional program to enhance antimicrobial stewardship. Clin Infect Dis, 44(2), 159-177 (2007). 
16. Kleinman LC, Norton EC. What's the Risk? A simple approach for estimating adjusted risk measures from nonlinear models including logistic regression. Health Serv Res, 44(1), 288-302 (2009).

17. Dunn G, Maracy M, Tomenson B. Estimating treatment effects from randomized clinical trials with noncompliance and loss to follow-up: the role of instrumental variable methods. Stat Methods Med Res, 14(4), 369-395 (2005).

18. Hulscher M, Prins JM. Antibiotic stewardship: does it work in hospital practice? A review of the evidence base. Clin Microbiol Infect, 23(11), 799-805 (2017).

19. Garin N, Genne D, Carballo S et al. beta-Lactam monotherapy vs beta-lactam-macrolide combination treatment in moderately severe community-acquired pneumonia: a randomized noninferiority trial. JAMA internal medicine, 174(12), 1894-1901 (2014).

20. Claessens YE, Debray MP, Tubach F et al. Early Chest Computed Tomography Scan to Assist Diagnosis and Guide Treatment Decision for Suspected Community-acquired Pneumonia. Am J Respir Crit Care Med, 192(8), 974-982 (2015).

21. Jacobs MR. Antimicrobial-resistant Streptococcus pneumoniae: trends and management. Expert Rev Anti Infect Ther, 6(5), 619-635 (2008). 


\section{SUPPLEMENTARY MATERIAL}

Table S1 Bacterial pathogens

\begin{tabular}{lllll}
\hline & \multicolumn{2}{c}{ Control (N = 2235) } & \multicolumn{2}{c}{ Intervention (N = 1849) } \\
\cline { 2 - 5 } & Proven & Possible & Proven & Possible \\
\hline Streptococcus pneumoniae & $205(9.1 \%)$ & $46(2.0 \%)$ & $188(10.2 \%)$ & $49(2.7 \%)$ \\
Staphylococcus aureus & $9(0.4 \%)$ & $32(1.4 \%)$ & $11(0.6 \%)$ & $45(2.4 \%)$ \\
Other gram-positives & $19(0.9 \%)$ & $18(0.8 \%)$ & $24(1.3 \%)$ & $5(0.3 \%)$ \\
Haemophilus influenzae & $4(0.2 \%)$ & $137(6.1 \%)$ & $7(0.4 \%)$ & $137(7.4 \%)$ \\
Moraxella catarrhalis & - & $20(0.9 \%)$ & - & $29(1.6 \%)$ \\
Escherichia coli & $17(0.8 \%)$ & $29(1.3 \%)$ & $13(0.7 \%)$ & $22(1.2 \%)$ \\
Klebsiella pneumoniae & $3(0.1 \%)$ & $11(0.5 \%)$ & $1(0.1 \%)$ & $18(1.0 \%)$ \\
Pseudomonas aeruginosa & $2(0.1 \%)$ & $35(1.6 \%)$ & - & $29(1.6 \%)$ \\
Other gram-negatives & $8(0.4 \%)$ & $65(2.9 \%)$ & $13(0.7 \%)$ & $78(4.2 \%)$ \\
Legionella pneumophila & $28(1.2 \%)$ & - & $22(1.2 \%)$ & - \\
Mycoplasma pneumoniae & - & - & - & $2(0.1 \%)$ \\
Mycobacteria & - & $2(0.1 \%)$ & - & $2(0.1 \%)$ \\
Aspergillus species & - & $16(0.7 \%)$ & - & $22(1.2 \%)$ \\
Candida species & - & $17(0.8 \%)$ & - & $25(1.4 \%)$ \\
Other fungi / yeast & - & $2(0.1 \%)$ & $1(0.1 \%)$ & $3(0.2 \%)$ \\
No pathogen & $811(36.3 \%)$ & $519(23.2 \%)$ & $650(35.2 \%)$ & $419(22.7 \%)$ \\
\hline Proven pathog)
\end{tabular}

Proven pathogens: based on pathogens detected in blood cultures, pleural fluid cultures, and urinary antigen tests (BINAX Now for S. pneumoniae and L. pneumophila). Possible pathogens: based on pathogens detected in sputum cultures, bronchoalveolar lavage fluid cultures, and serology. 
Table S2 Stewardship implementation

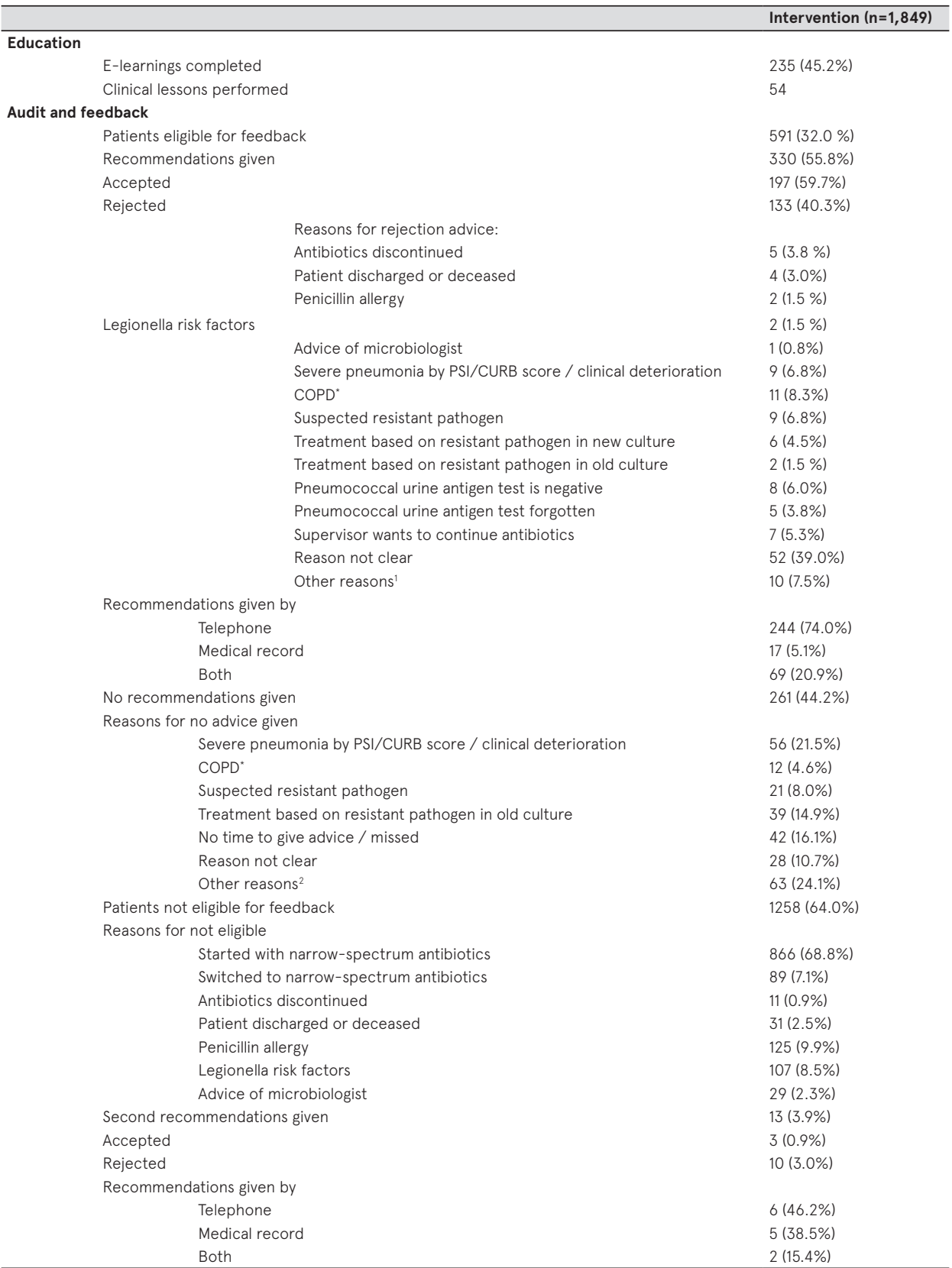

* Chronic Obstructive Pulmonary Disease, 1: Other reasons for rejecting advice; due to hospital-acquired pneumonia (not according to official risk factors), due to possible other focus, due to recurrent pneumonia, post-obstructive pneumonia, bronchiectasis or awaiting culture results.

2: Other reasons for no advice given: hospital-acquired pneumonia (not according to guideline risk factors), patient immunocompromised (not according to our definitions), suspected empyema, patient is agitated, possible other focus, awaiting culture results, post-obstructive pneumonia, due to legionella risk factors (not according to guideline risk factors), all cultures negative, possible abscess, continuing treatment of general practitioner. 
Table S3 Crude, adjusted for design and fully adjusted relative risk reductions in broad-spectrum DOT

\begin{tabular}{llllll}
\hline & $\begin{array}{l}\text { Control } \\
(\mathbf{n = 2 , 2 4 0 )} \\
\text { Median (IQR) }\end{array}$ & $\begin{array}{l}\text { Intervention } \\
(\mathbf{n = 1 , 8 4 4 )} \\
\text { Median (IQR) }\end{array}$ & $\begin{array}{l}\text { Crude RRR } \\
(\mathbf{9 5 \%} \mathrm{Cl})\end{array}$ & $\begin{array}{l}\text { Adjusted for design RRR* } \\
(\mathbf{9 5 \%} \mathrm{Cl})\end{array}$ & $\begin{array}{l}\text { Fully adjusted } \\
(\mathbf{9 5} \% \mathrm{Cl})\end{array}$ \\
\hline Broad-spectrum & $6(2-9)$ & $3(0-8)$ & $20.2 \%$ & $28.1 \%$ & $28.4 \%$ \\
DOT & & $(9.2 \%-29.8 \%)$ & $(15.5 \%-38.9 \%)$ & $(16.2 \%-38.9 \%)$ \\
\hline
\end{tabular}

* Adjusted for design and time, ${ }^{* *}$ also adjusted for possible confounders.

Table S4 Crude intervention effect stratified per hospital

\begin{tabular}{llllll}
\hline & \multicolumn{2}{l}{$\begin{array}{l}\text { Broad-spectrum DOT } \\
\text { (median, IQR) }\end{array}$} & & \multicolumn{2}{l}{ Empirical narrow-spectrum (proportion) } \\
\cline { 2 - 6 } & Control & Intervention & Adjusted risk difference & Control & Intervention \\
\hline Hospital & & & & & 28,1 \\
A & $4(1-9)$ & $4(1-9)$ & 0,225 & 29,2 & 52,0 \\
B & $4(0-8)$ & $3(0-8)$ & 0,170 & 47,9 & 69,1 \\
C & $2(0-7)$ & $0(0-5)$ & 0,167 & 59,2 & 27,3 \\
D & $8(3-9)$ & $5(2-9)$ & 0,286 & 14,7 & 43,6 \\
E & $8(3-11)$ & $3(0-8)$ & 0,393 & 16,2 & 49,5 \\
F & $4(0-8)$ & $3(0-8)$ & 0,219 & 39,1 & 48,3 \\
G & $8(4-11)$ & $2(0-8)$ & 0.376 & 20,5 & 60,0 \\
H & $6(0-9)$ & $3(0-8)$ & 0,259 & 31,8 & 63,6 \\
I & $7(2-9)$ & $2(0-8)$ & 0,284 & 26,7 & 45,9 \\
\hline Total & $6(2-9)$ & $3(0-8)$ & 0,284 & 28,8 & \\
\hline
\end{tabular}


Table S5 Specification of antibiotics given as empirical treatment

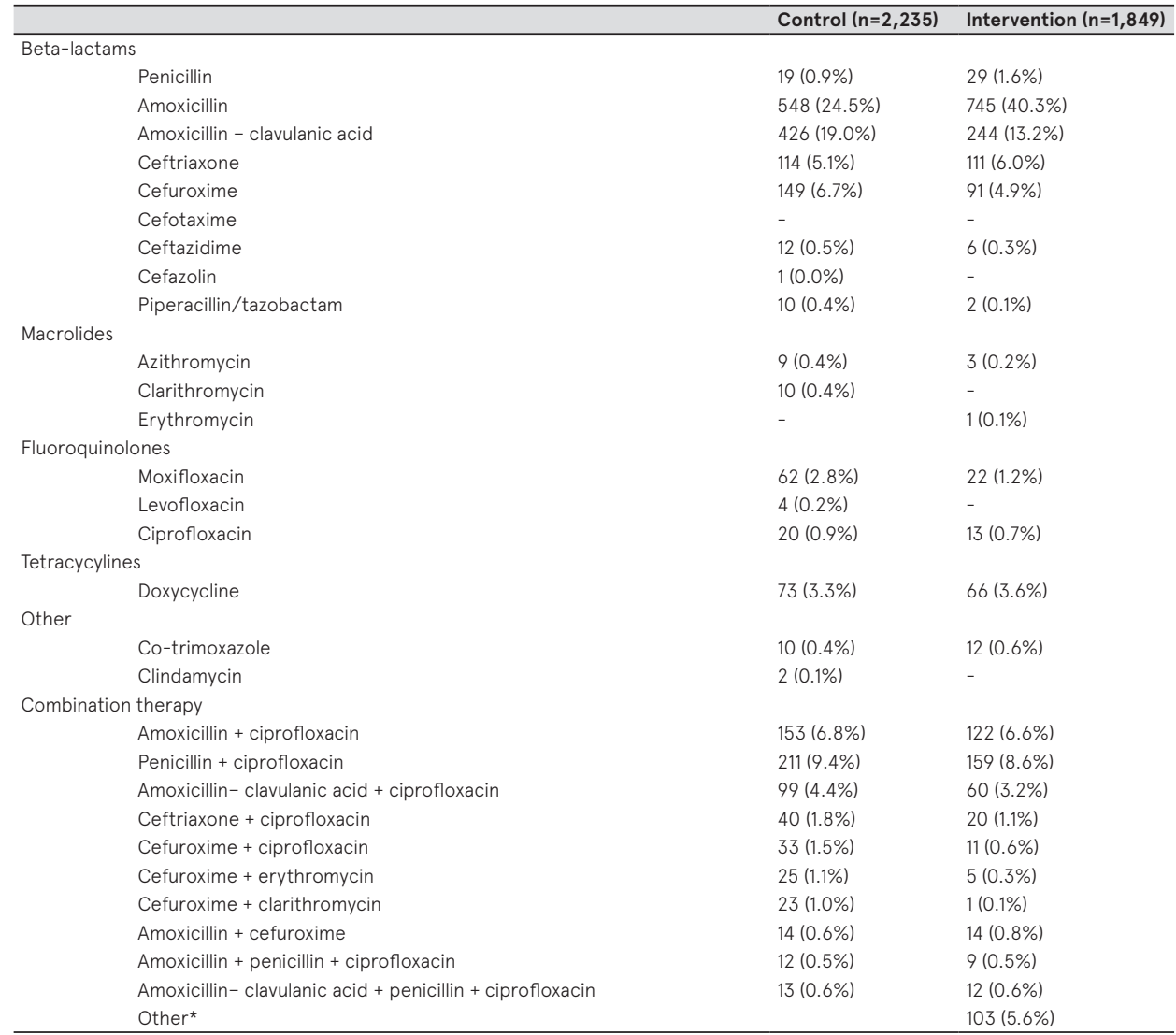

*All other combination therapies below $\mathrm{N}=10$. 
Table S6 Crude, adjusted for design and fully adjusted risk differences in 90-day mortality

\begin{tabular}{llllll}
\hline 90-day mortality & $\begin{array}{l}\text { Control } \\
(\mathbf{n = 2 , 2 4 0 )}\end{array}$ & $\begin{array}{l}\text { Intervention } \\
(\mathbf{n = 1 , 8 4 4 )}\end{array}$ & Crude RD & Adjusted for design RD* & Fully adjusted** RD \\
\hline $\mathrm{N}(\%)$ & $242(10.9 \%)$ & $199(10.8 \%)$ & & & \\
\hline Intention-to-treat & & $-0.09 \%(-1.7 \% ; 1.5 \%)$ & $0.9 \%(-2.3 \% ; 3.2 \%)$ & $0.4 \%(-2.7 \% ; 2.4 \%)$ \\
$90 \% \mathrm{Cl}$ & & $-0.09 \%(-2.0 \% ; 1.8 \%)$ & $0.9 \%(-2.9 \% ; 3.7 \%)$ & $0.4 \%(-3.3 \% ; 2.8 \%)$ \\
$95 \% \mathrm{Cl}$ & & & & \\
\hline $\mathrm{As}-\mathrm{treated}$ & & $-3.1 \%(-4.6 \% ;-1.4 \%)$ & $-3.1 \%(-4.8 \% ;-1.4 \%)$ & $0.07 \%(-1.8 \% ; 1.7 \%)$ \\
$90 \% \mathrm{Cl}$ & & $-3.1 \%(-4.9 \% ;-1.1 \%)$ & $-3.1 \%(-5.1 \% ;-1.1 \%)$ & $0.07 \%(-2.1 \% ; 2.1 \%)$ \\
$95 \% \mathrm{Cl}$ & & $-0.5 \%(-12.6 \% ; 10.1 \%)$ & $5.4 \%(-13.9 \% ; 19.1 \%)$ & $2.2 \%(-15.8 \% ; 13.7 \%)$ \\
\hline $\mathrm{CACE} * * *$ & & $-0.5 \%(-15.0 \% ; 12.1 \%)$ & $5.4 \%(-17.0 \% ; 22.7 \%)$ & $2.2 \%(-19.2 \% ; 16.4 \%)$ \\
$90 \% \mathrm{Cl}$ & & &
\end{tabular}

* Adjusted for design and time, ${ }^{* *}$ also adjusted for possible confounders, ${ }^{* *}$ Complier Average Causal Effect.

Table S7 Crude, adjusted for design and fully adjusted risk differences in 30-day mortality

\begin{tabular}{|c|c|c|c|c|c|}
\hline 30-day mortality & $\begin{array}{l}\text { Control } \\
(n=2,240)\end{array}$ & $\begin{array}{l}\text { Intervention } \\
(n=1,844)\end{array}$ & Crude RD & Adjusted for design RD* & Fully adjusted** RD \\
\hline $\mathrm{N}(\%)$ & $154(6.9 \%)$ & $123(6.7 \%)$ & & & \\
\hline \multicolumn{6}{|l|}{ Intention-to-treat } \\
\hline $90 \% \mathrm{Cl}$ & & & $-0.3 \%(-1.6 \% ; 1.1 \%)$ & $-0.7 \%(-2.9 \% ; 1.2 \%)$ & $-1.1 \%(-3.1 \% ; 0.7 \%)$ \\
\hline $95 \% \mathrm{Cl}$ & & & $-0.3 \%(-1.8 \% ; 1.3 \%)$ & $-0.7 \%(-3.3 \% ; 1.6 \%)$ & $-1.1 \%(-3.5 \% ; 1.1 \%)$ \\
\hline \multicolumn{6}{|l|}{ As-treated } \\
\hline $90 \% \mathrm{Cl}$ & & & $-2.6 \%(-4.0 \% ;-1.4 \%)$ & $-2.6 \%(-4.0 \% ;-1.4 \%)$ & $-0.3 \%(-1.8 \% ; 1.1 \%)$ \\
\hline $95 \% \mathrm{Cl}$ & & & $-2.6 \%(-4.2 \% ;-1.1 \%)$ & $-2.6 \%(-4.2 \% ;-1.1 \%)$ & $-0.3 \%(-2.0 \% ; 1.4 \%)$ \\
\hline \multicolumn{6}{|l|}{ CACE*** } \\
\hline $90 \% \mathrm{Cl}$ & & & $-1.5 \%(-9.1 \% ; 6.1 \%)$ & $-3.9 \%(-17.0 \% ; 7.6 \%)$ & $-6.4 \%(-18.5 \% ; 4.2 \%)$ \\
\hline $95 \% \mathrm{Cl}$ & & & $-1.5 \%(-10.6 \% ; 7.5 \%)$ & $-3.9 \%(-19.4 \% ; 10.0 \%)$ & $-6.4 \%(-20.8 \% ; 6.3 \%$ \\
\hline
\end{tabular}

* Adjusted for design and time, ${ }^{* *}$ also adjusted for possible confounders, ${ }^{* * *}$ Complier Average Causal Effect.

Table S8 Complications

\begin{tabular}{lll}
\hline & $\begin{array}{l}\text { Control }(\mathbf{n}=\mathbf{2 , 2 3 5 )} \\
\mathbf{n}(\%)\end{array}$ & $\begin{array}{l}\text { Intervention }(\mathbf{n}=\mathbf{1 , 8 4 9 )} \\
\mathbf{n}(\%)\end{array}$ \\
\hline Pleural effusion & $398(17.8)$ & $326(17.6)$ \\
Organ failure & $78(3.5)$ & $87(4.7)$ \\
Empyema & $22(1.0)$ & $23(1.2)$ \\
Septic shock & $12(0.5)$ & $4(0.2)$ \\
Acute respiratory distress syndrome & $10(0.5)$ & $7(0.4)$ \\
Pneumothorax & $9(0.4)$ & $3(0.2)$ \\
Lung abscess & $9(0.4)$ & $1(0.1)$ \\
Other & $244(10.9)$ & $205(11.1)$ \\
\hline
\end{tabular}




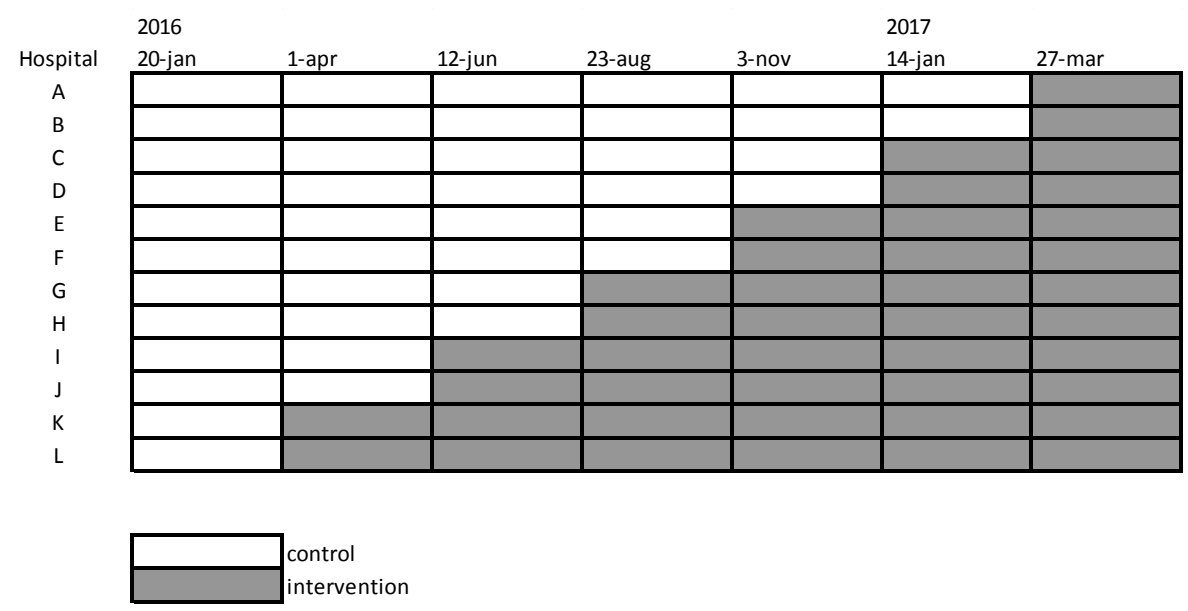

Figure $\mathbf{S} 1$ Stepped-wedge randomisation scheme

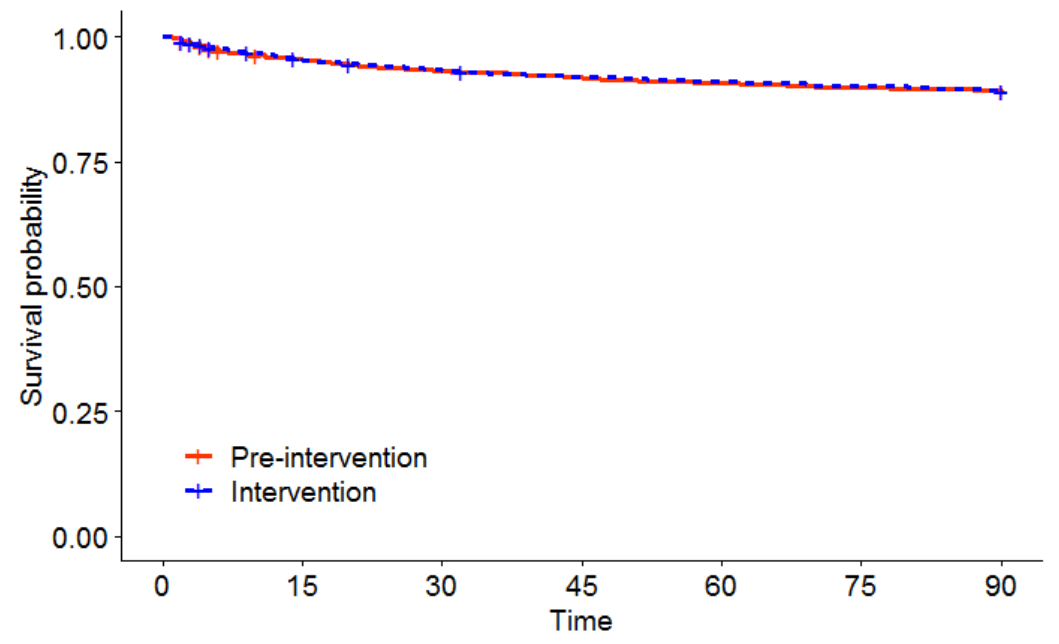

Figure S2 Kaplan Meier Curve 
abo

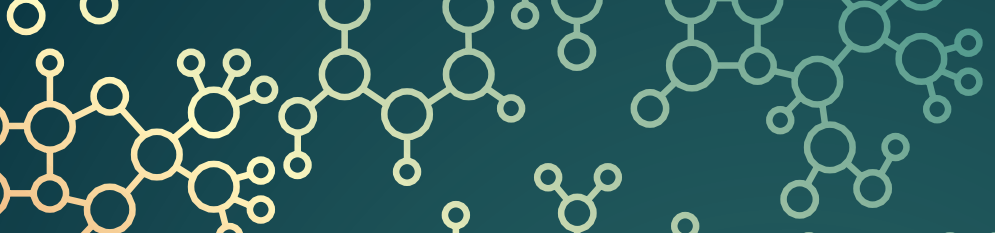
o

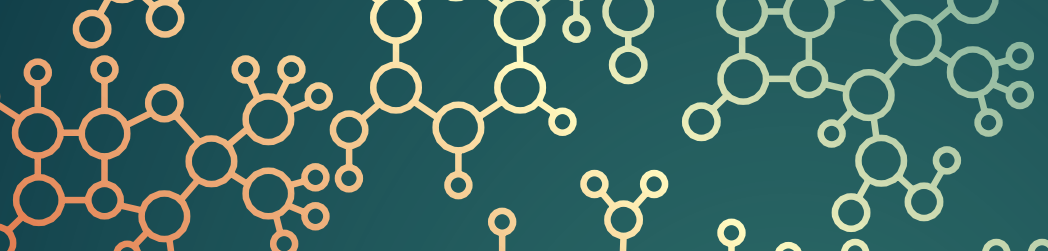

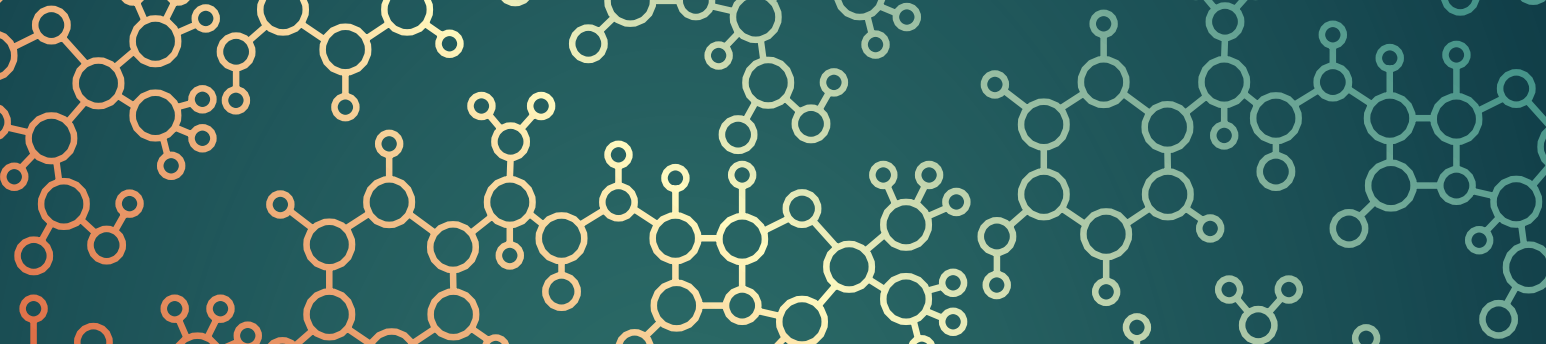

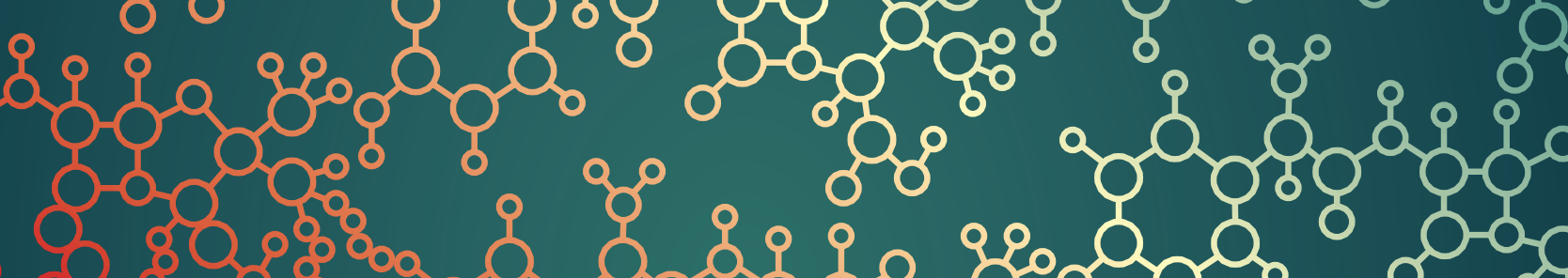
80\%

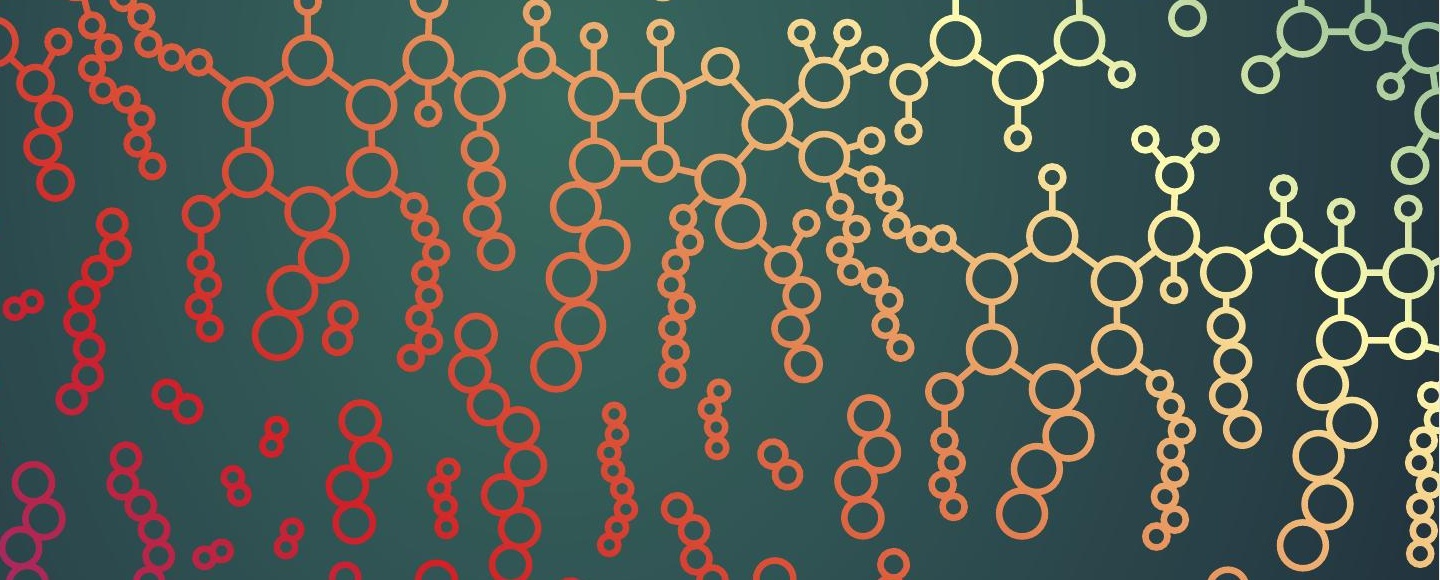
$88 \% 888$ $\circ 88$ \& 8

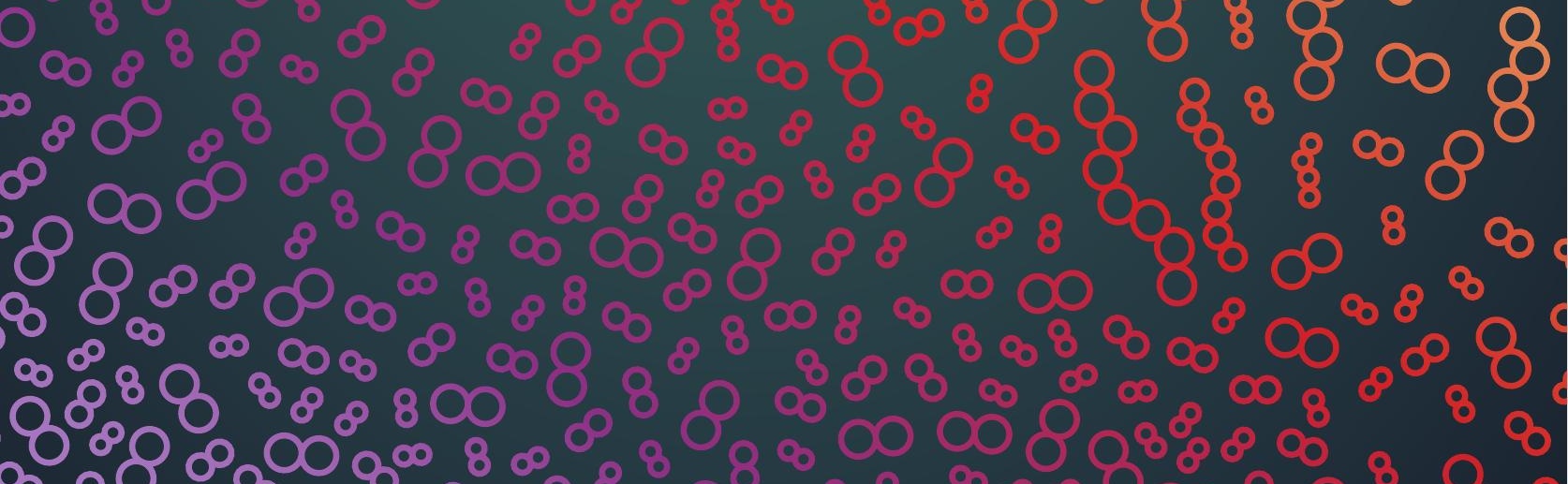




\section{Process evaluation of an}

effective antibiotic stewardship intervention in hospitalized patients with communityacquired pneumonia.

Inger van Heijl, Valentijn A. Schweitzer, Wendelien Dorigo-Zetsma, Cornelis H. van Werkhoven, Marlies E.J.L. Hulscher, Paul D. van der Linden, Marc J.M. Bonten, on behalf of the CAP-PACT study group 


\section{ABSTRACT}

\section{Introduction}

Community-acquired pneumonia (CAP) is a frequently occurring infection with high mortality. Adherence to empirical antibiotic treatment recommendations in clinical practice is low and many patients are treated with broad-spectrum antibiotics. One of the most important tools to improve adherence to guideline recommendations is through an antibiotic stewardship program (ASP). In a stepped-wedge cluster randomized trial (CAPPACT trial) a multifaceted stewardship intervention reduced the use of broad-spectrum antibiotics with $26.9 \%$ while the strategy was non-inferior for clinical outcome.

\section{Objectives}

Provide a detailed description of the delivered ASP and recommendations for ASP implementation for treatment of moderate-severe CAP.

\section{Methods}

We used data from the "Community-Acquired Pneumonia increasing Protocol adherence by Antibiotic stewardship in a stepped-wedge Cluster-randomized Trial" (CAP-PACT trial clinicaltrials.gov NCT02604628), which was performed in 9 Dutch hospitals between November 2015 and November 2017 and included 4084 patients with CAP. The multifaceted ASP intervention strategy consisted of education (clinical lessons, e-learning, pocket card and a poster), motivation opinion leaders and audit and feedback.

\section{Results}

A process evaluation was performed of the implementation strategy. The clinical lessons, use of pocket cards, motivation of opinion leaders and audit and feedback were considered the most effective intervention components. The content of the e-learning was good, however the clinicians experienced 'e-learning fatigue' reducing its perceived effectiveness. The content of the poster was not clear and was thereby less appreciated. For all intervention components, exposures, experiences and recommendations are provided.

\section{Conclusion}

It is recommended to implement regular clinical lessons including some form of benchmarking, distribute pocket cards, appoint and maintain actively involved local opinion leaders of the departments involved and perform daily audit and feedback. The proposed improvements may increase the efficacy of the ASP. 


\section{INTRODUCTION}

Community-acquired pneumonia (CAP) is an acute lower respiratory tract infection associated with high mortality and morbidity ${ }^{[1,2]}{ }^{2}$. In the Netherlands the recommended empirical therapy for moderate-severe CAP is amoxicillin or penicillin ${ }^{[2]}$. However, in clinical practice adherence to the national CAP guideline is low and many patients are unnecessary treated with broad-spectrum antibiotics ${ }^{[3,4]}$. Unnecessary use of broadspectrum antibiotics leads to an increase of antibiotic resistance, healthcare costs and complications ${ }^{[3]}$. Therefore, reducing excessive use of broad-spectrum antibiotics has been a major focus of antibiotic stewardship programs (ASPs) worldwide. ASPs aim to minimize unnecessary or inappropriate therapy and optimize outcomes ${ }^{[5]}$. Overall, antibiotic stewardship strategies have been shown to be effective tools to increase guideline adherence resulting in a reduction of broad-spectrum antibiotic use and adverse drug reactions. To assess if an antibiotic stewardship intervention was effective and safe in reducing the use of broad-spectrum antibiotics in the treatment of moderate-severe CAP the "Community-Acquired Pneumonia increasing Protocol adherence by Antibiotic stewardship in a stepped-wedge Cluster-randomized Trial" trial (CAP-PACT; clinicaltrials. gov NCT02604628) was performed. The aim of the current study is to provide a detailed description of the multifaceted ASP intervention and to provide recommendations for ASP implementation in other hospitals where ASP in moderate-severe CAP patient is needed.

\section{METHODS}

The CAP-PACT trial was a multicentre trial in 9 Dutch hospitals and lasted from November 2015 to November 2017. In the study 4,084 patients with a median age of 73 years were included; 2,235 patients in the control- and 1,849 in the intervention period. The multifaceted ASP consisted of a bundle of interventions i.e. A) education (clinical lessons, e-learning, pocket cards and posters), B) motivation by opinion leaders, and C) audit and feedback with the aim to change the prescribing behaviour of the physicians' into more guideline adherent. The ASP led to reduced broad spectrum antibiotic use by $26.9 \%$ (95\% $\mathrm{Cl}: 15.4 \%-37.4 \%)$ without negatively affecting patient outcomes. There was considerable heterogeneity between hospitals in reduction of broad-spectrum antibiotic use, with adjusted effect sizes ranging from $21 \%-37 \%$. To provide a detailed description of the ASP intervention and recommendations on how to implement this strategy, a process evaluation was performed of the implementation strategy. The process evaluation includes four steps: a description of (1) the intervention as planned, (2) the intervention 
as delivered, (3) the observed exposure to the intervention, and (4) recommendations to improve the intervention components. All intervention components of the ASP strategy were targeted at physicians (specialists and residents) of the Pulmonary and Internal medicine departments (target group). Each intervention component was classified according to the Effective Practice and Organisation of Care (EPOC) Taxonomy for Implementation strategies, category: interventions targeted at health care workers (Table 1) ${ }^{[6]}$. Per intervention component of the ASP strategy the process steps were evaluated.

Table 1 Intervention components of ASP strategy according to EPOC taxonomy.

\begin{tabular}{cl}
\hline Intervention components & EPOC subcategory \\
\hline Educational activities & \\
Clinical lessons & Educational meetings \\
& Monitoring the performance of the delivery of healthcare \\
E-learning & Educational materials \\
Pocket card & Reminders \\
Poster & Reminders \\
Activating opinion leaders & Local opinion leaders \\
Audit and feedback from antibiotic stewardship team & Audit and feedback \\
\hline
\end{tabular}

The 'intervention as planned' describes the intervention as defined a priori in the study protocol. How the 'intervention was delivered' was assessed by study parameters that were collected during the study (e.g. number and frequency of clinical lessons performed). The observed exposure was measured by means of attendance lists (clinical lessons), via Google Forms (e-learning), and by registration of whether a recommendation was given or not (audit and feedback). The observed exposure of using pocket cards, posters and the activity of opinion leaders was not measured. The recommendations to improve each intervention component were based on experiences of the participating physicians with the ASP. To assess this, semi-structured interviews with participating physicians were performed to gather experiences and recommendations for improvement. The interviews were conducted in December 2019, two years after ending data collection for the primary endpoints of the CAP-PACT trial. However, the ASP intervention is still actively carried out in eight of the participating hospitals: seven hospitals in the context of a follow-up study (the DiagNostic Study of Low-dose CT and multipleX PCR on Antibiotic Treatment and Outcome of Community-Acquired Pneumonia; CAP-NEXT. Clinicaltrials. gov NCT03360851), and one independently. In one hospital the ASP discontinued at the end of the CAP-PACT study period; this hospital was not included in the interviews. We interviewed physicians from 4 participating hospitals. We selected these 4 hospitals based on their antibiotic stewardship results to create the largest variety in hospitals (Table 2). Hospital (1) started as a moderately well performing hospital and the intervention yielded 
a moderate effect; hospital (2) started as the best performing hospital and still had a moderate decrease in broad-spectrum antibiotic use; hospital (3) started as a moderate performing hospital and had a large decrease in broad-spectrum antibiotic use; hospital (4) started as the worst performing hospital and had a large decrease in broad-spectrum antibiotic use. Within these hospitals, the principal investigators (PIs) and selected opinion leaders were invited for interviews by e-mail. The response to the invitations was $100 \%$. We originally planned eight interviews (two per hospital), and in response to these we scheduled one more interview in three hospitals. In total 11 physicians (3 Pulmonologists, 2 Internists, 4 Medical Microbiologists, 1 resident of the Microbiology department and 1 resident of the Pulmonology department) were interviewed by one researcher $(\mathrm{IH})$ at time and locations convenient to the participants within a period of two weeks. We used an interview guide (Appendix 1) to structure the interviews based on the framework for describing the key features of a strategy for change ${ }^{[7]}$ with the focus on experiences and suggestions for improvement of the intervention components. Two interviewees from different sites (one Medical Microbiologist and one resident of Medical Microbiology) declared they were only involved in the audit and feedback, thus they only received questions regarding this intervention component. A third person was interviewed in both of these hospitals. At last the participants were asked which (set of) components were in their opinion the most or the least effective in reducing broad-spectrum antibiotic use. To prevent social desirable responses all participants were encouraged to speak freely about the intervention. The interviews lasted on average 33 minutes (range 10 53 minutes). Interviews were voice-recorded and transcribed verbatim. Interviews were scheduled until data saturation was reached.

Transcripts were analysed using the thematic content approach. Data were collected and analysed concurrently, allowing emerging themes to be incorporated and explored in subsequent interviews. First, transcripts were read out in full, then data were coded based on the key features as mentioned above. Data were coded, organised and selected from transcripts using NVIVO 12.0. All transcripts were read carefully and analysed. Researcher $1(\mathrm{IH})$ assigned labels. To reduce inter-subjectivity of the coding process, a second researcher $(\mathrm{PL})$ verified whether the assigned labels covered all collected data. Differences were solved by discussion. 
Table 2 Characteristics and effects of hospitals participating in interviews.

\begin{tabular}{lllll}
\hline Hospital & $\begin{array}{l}\text { Characteristics } \\
\text { (No. of hospital beds) }\end{array}$ & \% effect* & $\begin{array}{l}\text { \% adherent therapy** } \\
\text { (control period) }\end{array}$ & $\begin{array}{l}\text { \% adherent therapy** } \\
\text { (intervention period) }\end{array}$ \\
\hline 1 & $\begin{array}{l}\text { Teaching hospital } \\
(500-750)\end{array}$ & $21.9 \%$ & $39.1 \%$ & $49.5 \%$ \\
2 & $\begin{array}{l}\text { Teaching hospital } \\
(500-750)\end{array}$ & $16.7 \%$ & $59.2 \%$ & $69.1 \%$ \\
3 & $\begin{array}{l}\text { General hospital } \\
(<500)\end{array}$ & $28.4 \%$ & $26.7 \%$ & $63.6 \%$ \\
4 & $\begin{array}{l}\text { Teaching hospital } \\
(500-750)\end{array}$ & $28.6 \%$ & $14.7 \%$ & $27.3 \%$ \\
\hline Total (all hospitals) & & $28.8 \%$ & $45.9 \%$ \\
\hline
\end{tabular}

* effect was measured as the average decrease in days of broad-spectrum antibiotics (DOTs) adjusted for confounders (PSI-score, smoking status, COPD, diabetes mellitus, and antibiotic pre-treatment). ${ }^{\star}$ Guideline adherent antibiotic therapy $=$ narrow-spectrum antibiotics (penicillin, amoxicillin, doxycycline).

\section{RESULTS}

\section{A) Education}

\section{$\underline{\text { Clinical lessons }}$}

Intervention as planned: The clinical lessons included theoretic information and casebased discussions on the national CAP guideline and feedback of antibiotic prescription data (measured until the moment of the lesson. These were planned to take place in 6-month intervals. This period was pragmatically chosen to balance sufficient frequency for reaching physicians who missed lessons due to night shifts and new employees, and to avoid irritation.

Intervention as delivered: Clinical lessons were developed in PowerPoint (See Appendix 2 for an example). Prescriber performance data of the intervention period compared to the control period was presented, and also anonymously benchmarked against other participating hospitals. The lessons were prepared and presented by the coordinating researchers (IH, VS and LZ). Time for preparation was approximately three hours. The lessons were scheduled approximately 3 months in advance, with help of the secretary of the department, and mostly scheduled at times already reserved for education. The lessons were scheduled every 3-4 months, more frequent than planned because 6 months was perceived as too infrequent to reach new employees and physicians that were absent. In total 54 lessons in 9 hospitals with a mean interval between the lessons of 108 days (3,9 months) were given. The first lesson (including the introduction of the study, the theory and the prescriber performance) lasted 30 minutes including time for discussion and questions. The follow-up lessons (including a short repetition of the theory and an update of prescription data and benchmarking) had a shorter duration of approximately 15 minutes. The actual duration was not measured but retrospectively 
estimated by the coordinating researchers. Attendance was registered during the first and either the second or third clinical lesson.

Observed exposure: The average attendance per lesson was 31\% (range of average per hospital: $11-53 \%$ ) and of the total target group, the proportion specialists versus residents was $28 \%$ and $72 \%$ respectively. After the first clinical lessons we handed out feedback forms to evaluate the content of the lessons and the way the content was presented (See Appendix 3 for an example) with a scoring system from $1-6(1=$ bad and $6=$ excellent $)$. In total 227 feedback forms were completed, with an average $( \pm S D)$ score of $4.3( \pm 0.7)$ for the extent of expectations for the lessons, a $4.1( \pm 0.9)$ for acquiring sufficient knowledge, insight or skills, a $4.4( \pm 0.7)$ for the quality of the lesson and a $4.5( \pm 0.9)$ for the practical use of the lesson. The average score was $4.7( \pm 0.6)$ for presentation skills and $4.8( \pm 0.7)$ for the degree of interaction with the participants. The participants perceived the presentation as useful, practical, clear and interesting. Other factors they appreciated were the short duration, the structure, the case based discussions, the presented results and the degree of interaction with the public. Feedback on suggestions for improvement were: aim was not clear, there was too much repetition of the content, change case based open questions into multiple-choice questions, increase difficulty level (especially for advanced residents and specialists), add more interaction, add more case-based questions and shorten the duration .

Experiences: In table 3 the experiences and suggestions for improvement provided during the in-depth interviews are summarized. Participants rated the clinical lessons with an average of $5.3( \pm 0.4)$. One of the most valued experiences was to schedule lessons at times already reserved for education. Also the benchmarking of the prescription data and the face-to-face contact during clinical lessons was very well appreciated.

Recommendations for improvement: The most important recommendations were first to also invite Emergency physicians for the clinical lessons in hospitals where they start empirical therapy of patients with CAP, secondly to announce the lessons with an attractive advertisement including the topic of the lesson, and thirdly that supervisors should motivate residents to attend the lessons (Table 3). 
Table 3 Experiences and suggestions for improvement per individual intervention component.

\begin{tabular}{|c|c|c|}
\hline Intervention components & Experiences & Suggestions for improvement \\
\hline \multicolumn{3}{|l|}{ (A) Educational activities } \\
\hline Clinical lessons & $\begin{array}{l}\text { - Target group was correct. } \\
\text { - Facilitators: schedule within a fixed education } \\
\text { moment and well ahead. } \\
\text { - Barriers: having clinical duty or a night-/ } \\
\text { weekend shift. } \\
\text { - Duration and frequency was sufficient. } \\
\text { - Content conveyed a clear message. } \\
\text { - Theoretic information too easy for specialists. } \\
\text { - Feedback of prescription performance } \\
\text { was most appreciated part and motivates to } \\
\text { perform better. } \\
\text { - Face-to-face contact was well appreciated. }\end{array}$ & $\begin{array}{l}\text { - Invite Emergency physicians' (when relevant) } \\
\text { - Nice advertising announcement beforehand (e.g. } \\
\text { results are presented) } \\
\text { - Supervisors should motivate residents to be present. } \\
\text { - Presenter should be knowledgeable on CAP and have } \\
\text { access to prescription data. } \\
\text { - Possibility to decrease frequency to once a year } \\
\text { (after implementation). }\end{array}$ \\
\hline Pocket card & $\begin{array}{l}\text { - Target group was correct. } \\
\text { - Facilitators: easy in use, it takes little time to } \\
\text { grab card out of pocket. } \\
\text { - Format and content were sufficient. } \\
\text { - Medium was well appreciated. } \\
\text { - Used by residents only vs. residents and } \\
\text { specialists (hospital depended) }\end{array}$ & $\begin{array}{l}\text { - Webpage scalable for mobile phones } \\
\text { - Digital PDF document }\end{array}$ \\
\hline $\begin{array}{l}\text { (B) Activating by } \\
\text { opinion leaders }\end{array}$ & $\begin{array}{l}\text { - Selected persons were active opinion leaders } \\
\text { by nature, by participation they became more } \\
\text { active. } \\
\text { - Active during hand-over meetings } \\
\text { Barriers: influence of policy from another } \\
\text { (academic) hospital). }\end{array}$ & $\begin{array}{l}\text { - Essential to have more than one active opinion } \\
\text { leader, ideally from every medical specialism. } \\
\text { - Organize a start meeting with all opinion leaders and } \\
\text { repeat this frequently to discuss possible issues. }\end{array}$ \\
\hline (C) Audit and feedback & $\begin{array}{l}\text { - Overall, the feedback was well received by } \\
\text { physicians and in good harmony. } \\
\text { - Majority of duration highly depended on the } \\
\text { audit part: screening for patients on Mondays } \\
\text { approx. } 60 \text { minutes. On Tuesdays till Fridays } \\
\text { approx. } 15 \text {-30 minutes. } \\
\text { - Few occasions lead to frustrating reactions, } \\
\text { but were always solved with a discussion. } \\
\text { - Feedback is given to physician that did not } \\
\text { prescribe empirical therapy. } \\
\text { - After a period the justification of the } \\
\text { empirical treatment was better recorded in } \\
\text { patients file. }\end{array}$ & $\begin{array}{l}\text { - It is essential that supervisors / local opinion leaders } \\
\text { are agreeing with the national guideline. } \\
\text { - Always register feedback in electronic patient file. } \\
\text { - Use an algorithm (clinical rule) to select CAP patients } \\
\text { then continuity is guaranteed. } \\
\text { - Use algorithm in electronic health care systems to } \\
\text { guide the appropriate empirical therapy. }\end{array}$ \\
\hline
\end{tabular}




\section{E-learning}

Intervention as planned: The e-learning consisted of theory and case-based questions on the national CAP guideline. It was planned to send the e-learning once a year to every participant and monthly to new employees.

Intervention as delivered: The e-learning was developed in Google Forms and consisted of 8 case-based questions (See Appendix 4 for an example). The coordinating researchers send an e-mail to the PIs which included the link to the e-learning with the request to forward this e-mail to the target group. The e-mail was forwarded by either an Internist, Pulmonologist, resident, research nurse or secretary of the department. The invitation was send approximately two weeks after the first clinical lesson and the coordinating researchers verified by e-mail to the principal investigator whether the invitation was send. Instead of monthly invitation for new employees and yearly invitations for all physicians, we sent invitations every 3-4 months to all employees directly after a clinical lesson, because the response rate to the first invitation was generally low. The content of the e-learning was the same as in the clinical lessons and was theorized to serve as a reminder and thus work synergistic to the clinical lessons. Response was monitored and at least once during the intervention period we send an update to each hospital with a list of the responders to the PI with the request to perform a follow-up on nonresponders. The duration to complete the e-learning was estimated beforehand by the coordinating researchers at approximately 10 minutes.

Observed exposure: Over the entire study period the e-learning was completed by 235/520 (45\%; range per hospital: $2.1-72 \%$ ) of the target group (37.1\% specialists vs. $62.9 \%$ residents).

Experiences: During the in-depth interviews participants rated the e-learning with an average score of $4.3 \pm(1.3)$. The content of the e-learning was very well appreciated. However, barriers for completing the e-learning are that participants receive a lot of invitations for completing e-learnings ('e-learning fatigue') and that they have a full mailbox in which the invitation quickly disappears (Table 3). The duration to complete the e-learning was estimated at 5-10 minutes and was valued as appropriate.

Recommendations for improvement: The most important recommendations were: first, to add accreditation or another obligation for completing the e-learning. Second, the intention was that the e-learning served as a booster, but the participants preferred if there were time reserved to complete the e-learning at the start of the clinical lesson or after a handover meeting. Finally, more frequent e-mails with proportion of responders 
send by a supervisor or another specialist to achieve a higher response (Table 3).

\section{Pocket cards}

Intervention as planned: The pocket cards were planned to be distributed at the start of the intervention period to the target group and to provide a short overview of the CAP guideline. The goal of the pocket cards was to serve as a quick reminder.

Intervention as delivered: The pocket cards were developed in Word and printed with plastic coating and sized A5 format in order to fit in a doctors coat pocket (See Appendix 5 for an example). At the start of the intervention, a number of pocket cards was printed based on at least one year of current and new employees. New pocket cards were printed on request. The content of the pocket card, a summary of the main recommendations of empirical treatment of CAP, was developed in collaboration with the principal investigators and the antibiotic stewardship team., and in some hospitals adjusted to local preferences. In some hospitals the pocket cards were used by specialists and residents, in other hospitals they were used only by the residents, according to local preference. Observed exposure: We did not measure whether pocket cards were handed over or used.

Experiences: During the in-depth interviews participants rewarded the pocket cards with an average score of $5.1( \pm 0.6)$. Pocket cards were very well appreciated because of the 'easiness in use'. In the general hospital the pocket card was used by specialists and residents alike, whereas in the teaching hospitals it was mainly used by residents.

Recommendations for improvement: The participants were very satisfied with the pocket cards. When asking if using a pocket card was valued in general, all participants agreed that they preferred this over an smartphone application, because it takes longer to search and open your app. In addition, for the development of an application at least 3 versions will be needed (iOS, Android, Windows) which would make the process expensive. It was suggested to develop a webpage (compatible with mobile phones) or a digitally accessible PDF document, although pocket cards were still preferred due to faster accessibility (Table 3).

\section{Posters}

Intervention as planned: The posters were planned to be distributed at the start of the intervention period to the target group and also provides a short overview of the treatment recommendations of CAP according to the national guideline. Similar to the pocket cards, the posters also served as a reminder. 
Intervention as delivered: The posters were developed in PowerPoint at a size of A4 and printed in colour on thick white glossy paper (See Appendix 6 for an example). Participants were requested to display the posters in the physician office, at the emergency room (ER), and at the pulmonology and internal medical ward.

Observed exposure: We did not measure whether and how visibly posters were displayed. Experiences: During the in-depth interviews most participants did not remember the poster. They scored the poster a $3.7( \pm 1.6)$ on average (after they had seen an example). Half of the participants thought the message was not clear, the other half thought the message was succinct. It was stated that a poster is very useful during studies, but participants were not convinced whether it would work in clinical practice (Table 3).

Recommendations for improvement: It was suggested to inquire upfront whether a hospital prefers to use a poster, the advice is to appoint someone who is responsible for the poster for putative updates or to check whether the poster is still visible, and to supply it on plastic coated paper for a longer durability (Table 3). Also it was recommended to adjust the content into a more clear message.

\section{B) Motivation by opinion leaders}

Intervention as planned: Opinion leaders of various departments were planned to be identified in collaboration with the local antibiotic stewardship team at the start of the intervention. In the Netherlands an antibiotic stewardship team is obligated in every hospital since January $2015^{[8]}$. These teams consist of at least one Medical Microbiologist, one Infectious Disease specialist, and one Hospital Pharmacist. The opinion leaders were planned to be selected based on their perceived influence on working behaviour during routine clinical practice. Meetings were planned to be scheduled with these local opinion leaders in 6 months intervals. During these meetings, past performances and barriers that impede adherence were planned to be discussed.

Intervention as delivered: At the start of the intervention, we asked local opinion leaders of the departments of Internal medicine, Pulmonology and Medical Microbiology (one from each department) to promote guideline adherent treatment throughout the intervention period, e.g. during hand-over meetings. The planned periodic meetings to discuss past performance and barriers that impede adherence were not performed. Instead, opinion leaders were mostly present during the clinical lessons during which past performance and barriers were discussed.

Observed exposure: It was not measured if and how frequently the opinion leaders 
promoted guideline adherence in practice.

Experiences: During the in-depth interviews the average score for this component was 3.8 ( \pm 1.0$)$. It was experienced that the identified opinion leaders were not equally active in promoting guideline adherence. This differed per hospital, and was experienced in hospital (1) by the opinion leader from one department being more active than the opinion leader from another department. In hospital (2) both opinion leaders valued each other as highly active and essential for the implementation of the intervention. In hospital (3) one opinion leader stated being very active, the other stated that opinion leaders are very important in implementing interventions. In hospital (4) it was expressed that the treatment of patients with CAP was to a large extend influenced by a near academic hospital that had a policy different from the national guidelines. The opinion leaders from this hospital needed discussions with each other to ensure adherence to the national guideline (Table 3).

Recommendations for improvement: The most important recommendations were first, to put more effort in having equal actively involved opinion leaders from all involved departments in antibiotic therapy in CAP patients (Internal medicine, Pulmonology and Microbiology), and second, to schedule regular meetings with the opinion leaders to discuss past performance and barriers that impede adherence (Table 3).

\section{C) Audit and feedback}

Intervention as planned: The prospective audit and feedback was performed by the local antibiotic stewardship team. Patients admitted with moderate-severe CAP were actively identified by screening admission charts during weekdays. If treatment was not according to guideline recommendations, and if this was not appropriately motivated in the medical charts, physicians were advised by a member of the local antibiotic stewardship team to switch treatment to penicillin or amoxicillin monotherapy. Screening of patients was only performed on week days. If, for any reason, treatment could not be switched to penicillin or amoxicillin monotherapy, it was recommended to perform a pneumococcal urine antigen test to facilitate de-escalation if the test result is positive. Recommendations were done by telephone and were registered in electronic health records.

Intervention as delivered: The positions of those providing the feedback differed per hospital (Table 4). Feedback was mostly given one day after empirical therapy was started because screening for patients was done once daily, often in the morning. In all hospitals, the screening and feedback was set up to be performed on all weekdays, however, in some hospitals there were short periods without feedback due to illness or holiday 
without replacement.

Observed exposure: A recommendation was given for a total of 330/591 (55.8\%) patients eligible for feedback. Reasons for accepting or rejecting the feedback, and reasons for not providing feedback to physicians' are presented in Table 5. The exact time to perform the audit and feedback was not measured.

Experiences: During the in-depth interviews the participants gave an average score 5.3 $( \pm 0.8)$. Overall the audit and feedback was well received. Negative reactions to feedback were rare and if it did occur it was solved quickly by discussion between both parties. It is perceived as a disadvantage that most of the time the feedback is given to the physician on duty the day of the audit. The person receiving the feedback (often a resident physician on duty at the ward) was most of the time not responsible for the empirical treatment of that patient (often the specialist or resident on duty at the ER the previous day). A negative experience was that supervisors disagreed with the feedback and stopped the residents from narrowing antibiotic therapy. Finally, the majority of the time spend on this intervention component was mainly due to the audit (screening) of patients. On Mondays screening included also the Weekend and lasted approximately 60 minutes. On other weekdays this was approximately 15-30 minutes (Table 3). Therefore, in some hospitals the audit was done by a different person than the person who provided the feedback.

Recommendations for improvement: The most important recommendation is that the specialists (supervisors, local opinion leaders) should agree with the policy to treat according to guideline. A second recommendation is to use an algorithm (clinical rule) to screen for CAP patients, which is less time consuming than actively screening admission charts. 
Table 4 Persons responsible for the audit and feedback

\begin{tabular}{|c|c|c|}
\hline \multirow{2}{*}{$\begin{array}{l}\text { Hospital } \\
1\end{array}$} & \multicolumn{2}{|c|}{ Audit and feedback performed by } \\
\hline & Audit: & research nurse of the Pulmonology department \\
\hline & Feedback: & medical microbiologist resident responsible for daily shift \\
\hline \multirow[t]{2}{*}{2} & Audit: & hospital pharmacist* \\
\hline & Feedback: & medical microbiologist responsible for daily shift \\
\hline \multirow[t]{2}{*}{3} & Audit: & medical microbiologist* responsible for daily shift \\
\hline & Feedback: & medical microbiologist* responsible for daily shift \\
\hline \multirow[t]{2}{*}{4} & Audit: & research nurse of the Microbiology department \\
\hline & Feedback: & medical microbiologist resident responsible for daily shift \\
\hline \multirow[t]{2}{*}{5} & Audit: & pulmonologist* \\
\hline & Feedback: & hospital pharmacist responsible for daily shift \\
\hline \multirow[t]{2}{*}{6} & Audit: & hospital pharmacist* \\
\hline & Feedback: & hospital pharmacist* \\
\hline \multirow[t]{2}{*}{7} & Audit: & research nurse ${ }^{*}$ of the Microbiology department \\
\hline & Feedback: & research nurse* of the Microbiology department \\
\hline \multirow[t]{2}{*}{8} & Audit: & research physician* of the Microbiology department \\
\hline & Feedback: & research physician* of the Microbiology department \\
\hline \multirow[t]{2}{*}{9} & Audit: & research nurse* of the Clinical Pharmacy department \\
\hline & Feedback: & research nurse* of the Clinical Pharmacy department \\
\hline
\end{tabular}

* These persons were part of the local antibiotic stewardship team.

Table 5 Audit and feedback

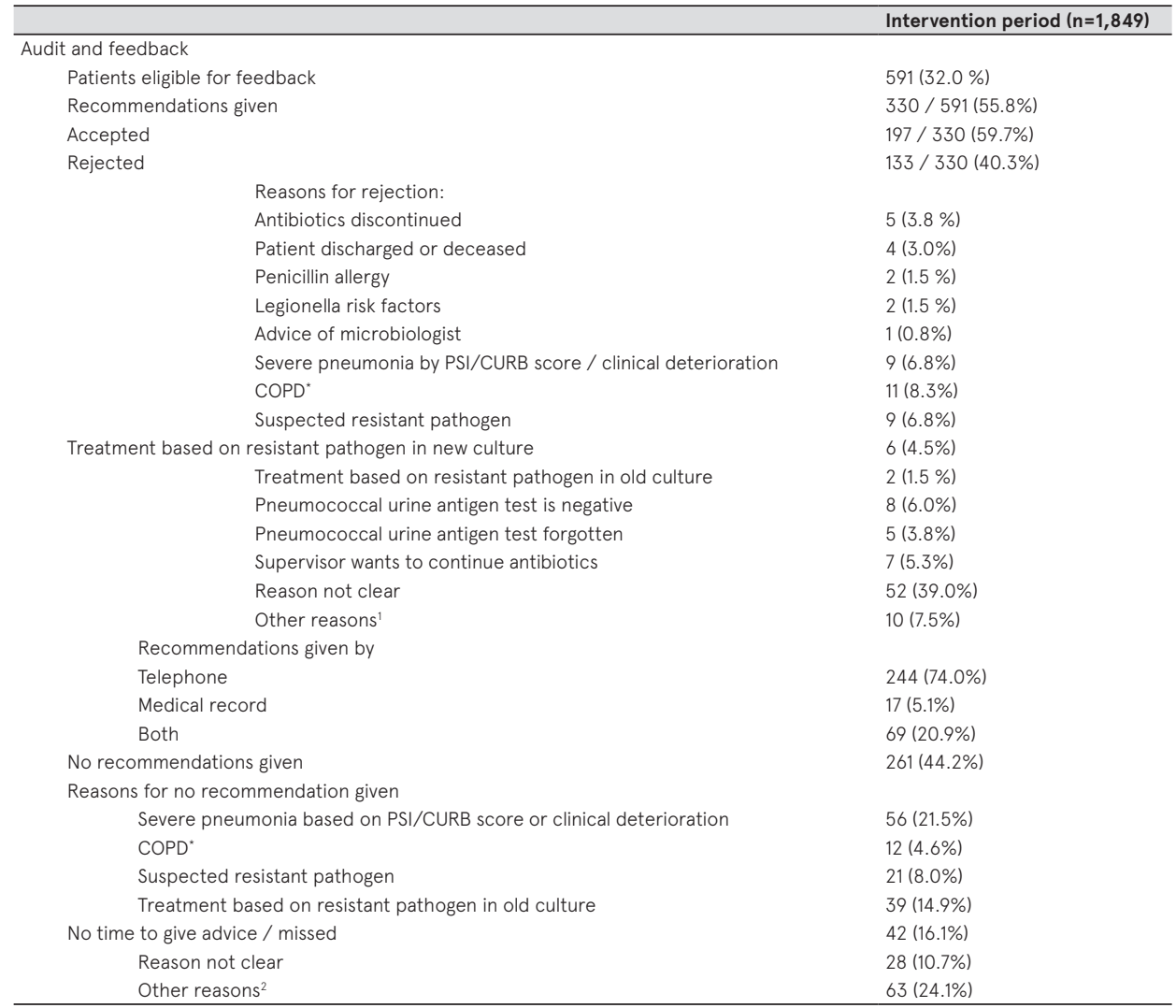


Table 5 Continued.

\begin{tabular}{|c|c|c|}
\hline & & Intervention period $(n=1,849)$ \\
\hline \multicolumn{3}{|l|}{ Audit and feedback } \\
\hline \multicolumn{2}{|c|}{ Patients not eligible for feedback } & $1258(64.0 \%)$ \\
\hline \multicolumn{3}{|c|}{ Reasons for non-eligibility } \\
\hline \multicolumn{2}{|r|}{ Started with narrow-spectrum antibiotics } & $866(68.8 \%)$ \\
\hline \multicolumn{2}{|r|}{ Switched to narrow-spectrum antibiotics } & $89(7.1 \%)$ \\
\hline \multicolumn{2}{|r|}{ Antibiotics discontinued } & $11(0.9 \%)$ \\
\hline \multicolumn{2}{|r|}{ Patient discharged or deceased } & $31(2.5 \%)$ \\
\hline \multicolumn{2}{|r|}{ Penicillin allergy } & $125(9.9 \%)$ \\
\hline \multirow{2}{*}{\multicolumn{2}{|c|}{ Legionella risk factors }} & $107(8.5 \%)$ \\
\hline & & $29(2.3 \%)$ \\
\hline \multicolumn{3}{|c|}{ Advice of microbiologist } \\
\hline \multirow{2}{*}{\multicolumn{2}{|c|}{ Second recommendations given }} & $13(3.9 \%)$ \\
\hline & & $3(0.9 \%)$ \\
\hline \multicolumn{3}{|l|}{ Accepted } \\
\hline & & $10(3.0 \%)$ \\
\hline \multicolumn{3}{|l|}{ Rejected } \\
\hline \multicolumn{3}{|c|}{ Second recommendations given by } \\
\hline & Telephone & $6(46.2 \%)$ \\
\hline & Medical record & $5(38.5 \%)$ \\
\hline & Both & $2(15.4 \%)$ \\
\hline
\end{tabular}

* Chronic Obstructive Pulmonary Disease, 1: Other reasons for rejecting advice; due to hospital-acquired pneumonia (not according to official risk factors), due to possible other focus, due to recurrent pneumonia, post-obstructive pneumonia, bronchiectasis or awaiting culture results. 2: Other reasons for no advice given: hospital-acquired pneumonia (not according to guideline risk factors), patient immunocompromised (not according to our definitions), suspected empyema, patient is agitated, possible other focus, awaiting culture results, post-obstructive pneumonia, due to legionella risk factors (not according to guideline risk factors), all cultures negative, possible abscess, continuing treatment of general practitioner.

\section{Comparative effectiveness of intervention components}

The clinical lessons, audit and feedback, and local opinion leaders were indicated as most effective intervention components of the ASP according to the participants. Clinical lessons were appreciated by all hospitals and all departments. The audit and feedback was appreciated by three hospitals (teaching and general), and by different departments (both by feedback givers and receivers). At last the opinion leaders were also indicated as most effective in three hospitals (teaching and general) and different by both opinion leaders and non-opinion leaders. The e-learning and the poster were indicated as the least effective intervention components. The e-learning was indicated as least effective by three hospitals (teaching and general) and only by specialists. The poster was less appreciated by all hospitals and all departments.

Hospital (1) started as a moderately well performing hospital and the intervention yielded a moderate effect. Three physicians were interviewed and expressed the following experiences: First, two participants valued clinical lessons and activating opinion leaders as most effective intervention component. Second, one participant stated that the opinion leader of their department was highly active, but similar to before the study. Third, this active opinion leader stated that the opinion leader of one other department was less 
active. Fourth, the participant who provided the feedback stated that communication with all residents and specialists of other departments always went smoothly. Finally, the participants valued e-learning and posters as least effective especially in clinical practice. Hospital (2) started as the best performing hospital and still had a moderate decrease in broad-spectrum antibiotic use. Two physicians were interviewed and expressed the following experiences: First, during the interviews both participants valued each other as a very active opinion leader, and one valued this also as one of the most effective intervention component. Second, both valued as most effective intervention components the clinical lessons and the audit-and feedback. Third, this hospital automated the audit (screening for eligible patients). This automated audit is an algorithm running once daily and generates a list with patients presumed having a CAP and receiving non-guideline adherent antibiotics. They implemented this for the sustainably of the intervention, because in this hospital the intervention is still actively implemented (without participation in a study). However, this the audit and feedback was the only component which maintained. Finally, both participants valued the poster as least effective.

Hospital (3) started as a moderate performing hospital and had a large decrease in broad-spectrum antibiotic use. Three physicians were interviewed and expressed the following experiences; First, all participants valued the clinical lessons as most effective intervention component. Second, two valued the audit and feedback also being the most effective component (both by receiver and giver of feedback). Third, one participant valued opinion leaders as the most effective component next to the lessons. Fourth, this general hospital has a small number of specialists and residents which results in less different opinions, alongside with short lines of communication. Finally, two participants valued the e-learning and one valued the poster as least effective.

Hospital (4) started as the worst performing hospital, had a large decrease in broadspectrum antibiotic use, but ended as the worst performing hospital as well. Three physicians were interviewed and expressed the following experiences; First, one participant valued the clinical lessons as most effective (also the feedback giver). Second, the other participant valued the audit and feedback as most effective intervention component (feedback receiver). Third, this hospital experienced influence from a near academic hospital that had a different policy from the national guideline, so that might have explained the remaining low adherence to the guideline in the intervention period. Finally, one participant valued the e-learning and the other one the poster as least effective. 


\section{DISCUSSION}

In hospitalized patients with CAP a reduction of $27 \%$ in broad-spectrum antibiotic use was achieved by implementing a multifaceted antibiotic stewardship interventions targeted to change the prescribing behaviour of physicians'. A detailed description of the process evaluation of different intervention components was provided and can be used by hospitals where there is a need for an ASP in CAP.

The clinical lessons were largely delivered as planned, except we scheduled the lessons more frequently than planned. Despite an exposure of only $30 \%$, this was one of the most valued components of the intervention (measured directly after the clinical lessons and two years later during the interviews). However, the lessons were only valued by the participants being present, which could bias the overall appreciation. Also, the exposure of $30 \%$ could be biased because it was only measured during the first lessons; participation rates may have been different at later times. Of the content, particularly the benchmark results and the case-based discussions were well appreciated. It is recommended to schedule lessons during moments already reserved for education and to invite also Emergency physicians. Supervisors should motivate residents to attend the clinical lessons. At last, the preparation of a clinical lesson was approximately 3 hours, which makes this a relatively time consuming intervention component. The e-learning was also delivered as planned, except for the frequency to send the invitations, this was adjusted to a more convenient moment (after clinical lessons). The participants appreciated the content, but because of an overload of e-learning invitations this component had an average lower score compared to the lessons. The pocket cards received similar average scores as the clinical lessons during the interviews. These were also delivered as planned, but the users differed by hospital (only by residents in teaching hospitals versus by residents and specialists in general hospital). It is a very easy-to-perform intervention component. The poster was not delivered as planned. None of the interviewees remembered the poster. It is uncertain whether and how long the posters were displayed and it was less appreciated by the participants compared to the lessons and pocket cards. It is also a very easy-to-perform intervention component, thus for hospitals that use posters frequently, this intervention can still be advised. However, it is recommended to adjust the content to a very clear message and to check for required updates on a regular basis. The local opinion leaders were not completely delivered as planned: meetings to evaluate past performances and discuss barriers that impede adherence were not performed. Such meetings were particularly suggested as an improvement by the participants. This component was, in combination with the clinical lessons and audit and feedback, classified as most effective intervention components. At last, the audit and feedback 
was delivered as planned. Despite that physicians received feedback on only half of the eligible patients, this was, according to the participants, an effective and well appreciated intervention component. However, when implementing this intervention component, it takes 15-60 minutes a day. For implementation, supervisors or local opinion leaders should agree to adhere to the guideline. It is suggested to use algorithms to select the patients targeted for audit and feedback.

To our knowledge, limited literature is available on how stewardship interventions are actually implemented in detail [9]. Published ASP studies report an effect, but do not provide a detailed description of their intervention. However, generalizability of ASP studies to a great extend depend on the quality of implementation of the different intervention components. Thus, there is a need for ASP studies to provide information regarding the implementation of the intervention, since this information can be used to understand and explain the main effect or the variety in effects between different participating centres. Also it can be used to compare effects between ASP studies, e.g. in case of systematic reviews. Finally and importantly, a detailed description of the intervention provides a great opportunity to implement the intervention locally. One of the prioritized research areas by the Working Group on Behavioral Approaches to Antibiotic Stewardship Programs is 'to conduct robust evaluations of ASPs with built-in process evaluations and fidelity assessments' ${ }^{[10]}$. This was also advised by a Cochrane review on ASP interventions ${ }^{[5]}$. Our study contributes to a better understanding of how the ASP intervention components were implemented, how they were received and how they might be improved.

In our study the ASP intervention was implemented as a one-size-fits all and this resulted overall in a reduction of $27 \%$ on broad-spectrum antibiotic use. However different effects per hospitals were achieved (range: 17\% - 39\%). It has been suggested that customized ASPs yield a larger effect compared to one-size-fits-all approaches ${ }^{[9]}$. In retrospect, we could have invested more time in discussing upfront with the participating hospitals how to offer the e-learning to gain a maximum response, and the usage and content of the poster. We could have scheduled frequent meetings with all opinion leaders together. Yet, it is uncertain to what extend a more customized approach would have increased the impact of the ASP.

Different facilitators and barriers may have played a role in the impact of the ASP in the four hospitals. In hospital (1) the lack of change in activity of opinion leaders may have prevented a larger effect. In hospital (2) the effect of the intervention was substantial despite a high guideline adherence at the start. This might be explained by the highly 
active opinion leaders. In hospital (3) the short lines of communication between physicians might be responsible for the effect. Finally, in hospital (4) the influence of a near academic hospital and the contrasting opinions within the hospital may have been a barrier.

A limitation of this study is that not all data for a process evaluation was collected, such as the exact time for preparation of clinical lessons, the exposure to all clinical lessons, exposure to pocket cards and posters and the actual performance of local opinion leaders. However, the data presented are of sufficient detail to understand the caveats and key drivers of a successful ASP and to facilitate implementation elsewhere.

To conclude, for hospitals that want to achieve a reduction in broad-spectrum antibiotic use in patients with CAP it is recommended to implement regular clinical lessons including some form of benchmarking, distribute pocket cards, appoint and maintain actively involved local opinion leaders of the departments involved and perform daily audit and feedback.

\section{List of abbreviations}

ASP, antibiotic stewardship program; CAP, Community-acquired pneumonia; CAP-PACT trial, Community-Acquired Pneumonia increasing Protocol adherence by Antibiotic stewardship in a stepped-wedge Cluster-randomized Trial; ER, Emergency Room; PI, Principal investigator.

\section{Acknowledgements}

We gratefully acknowledge Lufang Zhang (University Medical Centre Utrecht, the Netherlands) for preparing and presenting some of the clinical lessons.

\section{Funding}

No financial support was received for this study.

\section{Conflict of interest}

The authors declare no conflict of interest. 


\section{REFERENCES}

1. L.M. Bjerre, T.J. Verheij, M.M. Kochen, Antibiotics for community acquired pneumonia in adult outpatients, Cochrane Database Syst Rev (4) (2009) CD002109

2. W.J. Wiersinga, M.J. Bonten, W.G. Boersma, R.E. Jonkers, R.M. Aleva, B.J. Kullberg, J.A. Schouten, J.E. Degener, E.M.W. van de Garde, T.J. Verheij, A.P.E. Sachs, J.M. Prins, Management of community-acquired pneumonia in adults: 2016 guideline update from the Dutch Working Party on Antibiotic Policy (SWAB) and Dutch Association of Chest Physicians (NVALT), Neth J Med 76(1) (2018) 4-13.

3. N. Adler, H. Weber, I. Gunadasa, A. Hughes, N. Friedman, Adherence to therapeutic guidelines for patients with community-acquired pneumonia in Australian hospitals, Clin Med Insights Circ Respir Pulm Med 8 (2014) 17-20.

4. S.M. Huijts, C.H. van Werkhoven, W.G. Boersma, J. Buijs, G. Buunk, C.J. Compaijen, L.J. van Elde, J.E. Gisolf, R. van der Kam, J.A. Kluytmans, B.A. Kuipers, J.J. Mager, B. Oppedijk, F. Palmen, J.M. Prins, B. van Reemst, M.H. Silbermann, F.H. van Tiel, E. van der Wall, T.S. van der Werf, M.J. Bonten, Guideline adherence for empirical treatment of pneumonia and patient outcome. Treating pneumonia in the Netherlands, Neth $\mathrm{J}$ Med 71(10) (2013) 502-7.

5. P. Davey, C.A. Marwick, C.L. Scott, E. Charani, K. McNeil, E. Brown, I.M. Gould, C.R. Ramsay, S. Michie, Interventions to improve antibiotic prescribing practices for hospital inpatients, Cochrane Database Syst Rev 2 (2017) Cd003543.

6. Effective Practice and Organisation of Care (EPOC), EPOC Taxonomy. <epoc.cochrane.org/epoc-taxonomy>, 2015 (accessed December 2, 2019.)

7. M. Hulscher, M. Laurant, R. Grol, Process evaluation of implementation strategies, in: R. Grol, M. Wensing, M. Eccles, D. Davis (Eds.), Improving patient care: the implementation of change in health care, Wiley Blackwell, Oxford, 2013, pp. 333-349.

8. The Dutch Working Party on AntibioticPolicy (SWAB), Antimicrobial Stewardship. <https://swab.nl>, 2016 (accessed January 30.2020).

9. M. Hulscher, J.M. Prins, Antibiotic stewardship: does it work in hospital practice? A review of the evidence base, Clin Microbiol Infect 23(11) (2017) 799-805

10. M. Rzewuska, E. Charani, J.E. Clarkson, P.G. Davey, E.M. Duncan, J.J. Francis, K. Gillies, W.V. Kern, F. Lorencatto, C.A. Marwick, J. McEwen, R. Mohler, A.M. Morris, C.R. Ramsay, S. Rogers Van Katwyk, B. Skodvin, I. Smith, K.N. Suh, J.M. Grimshaw, P. Joint Programming Initiative on Antimicrobial Resistance Working Group on Behavioural Approaches to Antibiotic Stewardship, Prioritizing research areas for antibiotic stewardship programmes in hospitals: a behavioural perspective consensus paper, Clin Microbiol Infect 25(2) (2019) 163168 


\section{SUPPLEMENTARY MATERIAL}

Appendix 1 Themes used in interview guide

\begin{tabular}{ll}
\hline Key features & Themes (experiences and suggestions for improvement) \\
\hline Target group & Appropriateness of the target group \\
& Barriers and facilitators for participation \\
Implementer & Appropriateness of presenting professionals \\
Intensity & Frequency, duration, timing. \\
Information imparted & Quality of content, presentation form, type of information, medium. \\
Information about target group performance & Quality of content, presentation form, type of information, medium. \\
\hline
\end{tabular}


Appendix 2 Example of a clinical lesson

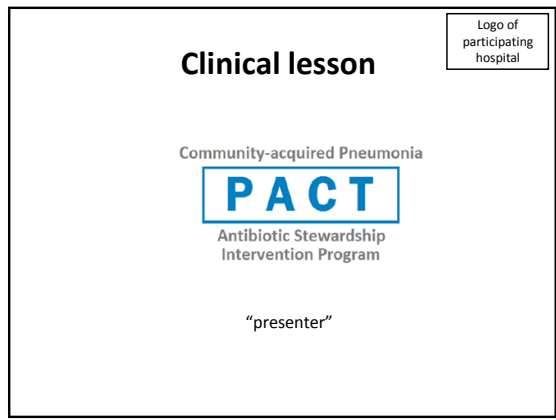

\section{Content}

- Introduction

- SWAB CAP guideline and questions

- Results; how is a CAP treated in clinical practice?

- Intervention

Introduction
Community-acquired pneumonia
Pneumonia acquired outside the hospital
Hospital-acquired pneumonia
Pneumonia $>48 \mathrm{hr}$ after hospital admission

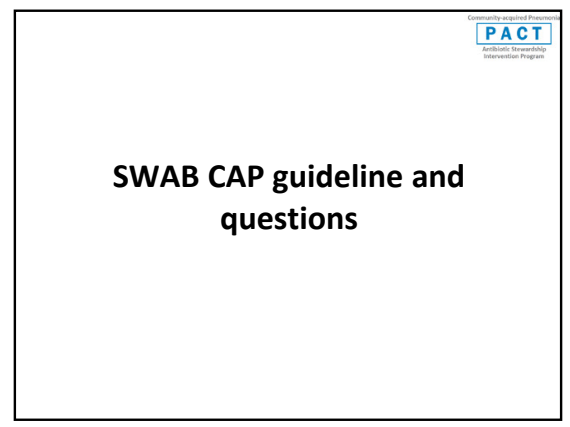

SWAB CAP guideline (question)
A 67-year-old man is admitted to the Pulmonology department with a
working diagnosis of CAPP. To start the appropriate empirical treatment,
you first determine the disease-severity. You can choose from one of the
three scoring systems; PSI score, CURB-65 score or pragmatic
classification.
Is there a preferred scoring system according to the guideline to
determine disease-severity of CAP?

\section{SWAB CAP guideline (question)}

A 67-year-old man is admitted to the Pulmonology department with a working diagnosis of CAP. To start the appropriate empirical treatment you first determine the disease-severity. You can choose from one of the three scoring systems; PSI score, CURB-65 score or pragmatic classification.

Is there a preferred scoring system according to the guideline to determine disease-severity of CAP?

No 

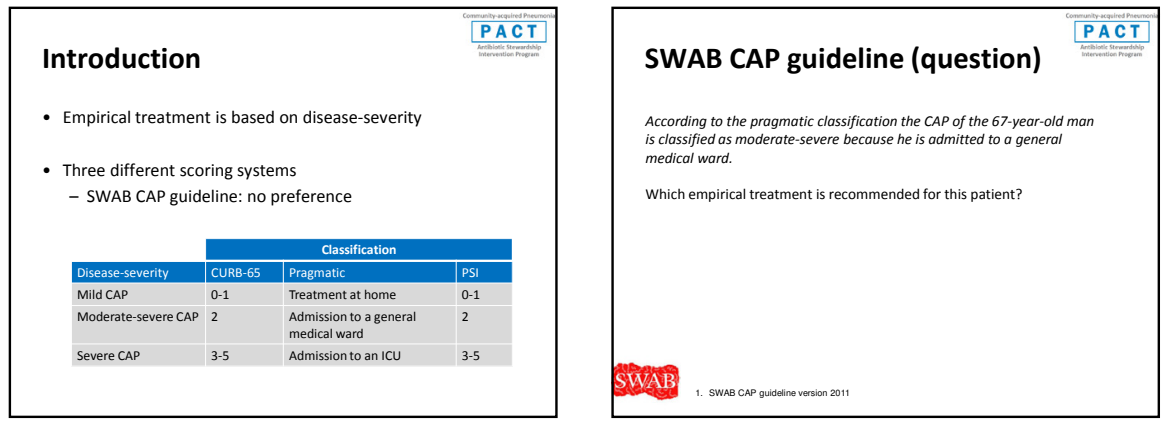

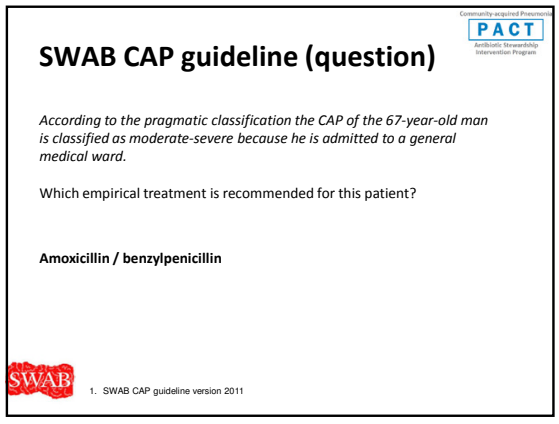
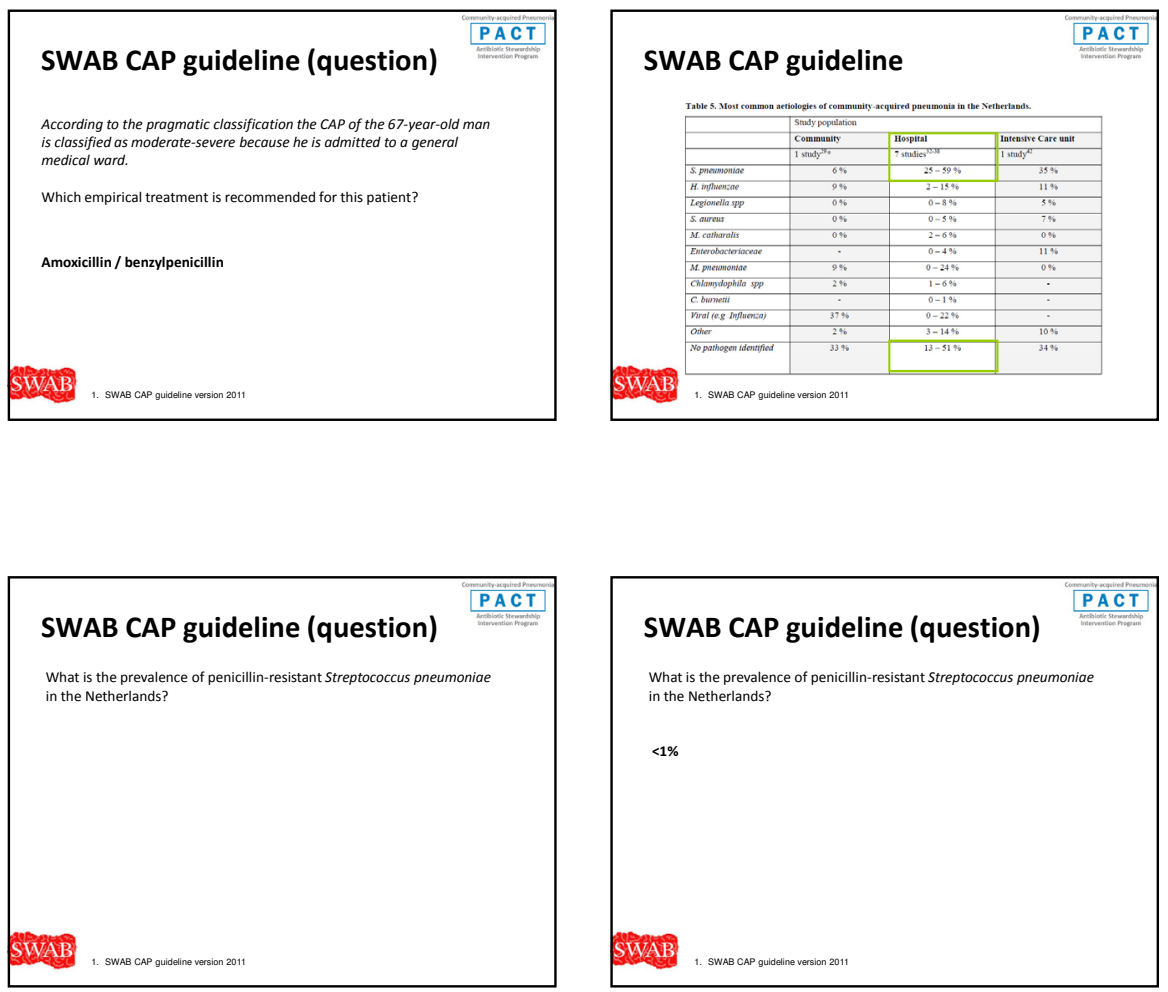

\section{SWAB CAP guideline (question)}

What is the prevalence of penicillin-resistant Streptococcus pneumoniae in the Netherlands?

$<1 \%$ 


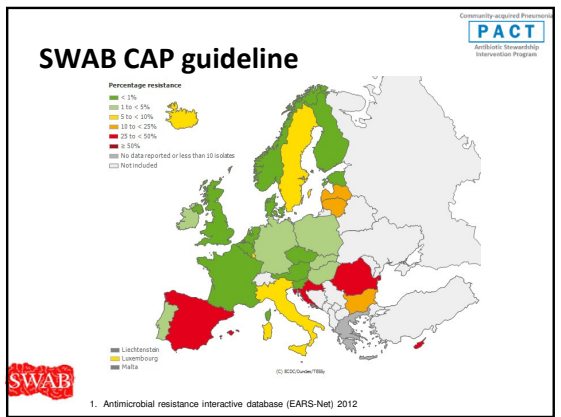

\section{SWAB: CAP richtlijn (question)}

Prior to the admission at the Pulmonology department a colleague at the

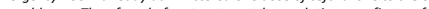
year-old man. Therefore, before treatment de escalation, you fist perform

What is the positive predictive value of this test?

$89-97 \%$

\section{SWAB: CAP richtlijn (question)}

P A C T

Prior to the admission at the Pulmonology department a colleague at the Emergency Room already administered one dose of ceftriaxone to the 67

year-old man. Therefore, before treatment de-escalation, you first perform

the pneumococcal urine antigen test. What is the positive predictive value of this test?

What is the positive predictive value of this test?

1. SWAB CAP guldeline version 2011

\section{SWAB CAP guideline (question)}

The turnaround time of a pneumococcal urine antigen test is 15 minutes. One hour after you performed this test, you phone the Microbiology department. The test result is negative. In the meantime, your 67-year-old patient does not improve after 1 day of treatment with ceftriaxone. You consider adding ciprofloxacin to the initial therapy to cover a possible Legionella infection.

In which situation is it appropriate to add antibiotic treatment for Legionella infection?

SWAB CAP guideline (question)
The turnaround time of a pneumococcal urine antigen test is 15 minutes. One
hour after you performed this test, you phone the Microbiology department.
The test result is negative. In the meantime, your 67-year-old patient does not
improve after 1 day of treatment with ceftriaxone. You consider adding
ciprofloxacin to the initial therapy to cover a possible Legionella infection.
In which situation is it appropriate to add antibiotic treatment for Legionella
infection?
Failure to improve despite 248 hours treatment with a beta-lactam
antibiotic at adequate dosage.
SWWAB

SWAB CAP guideline

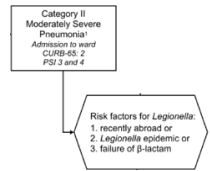

Syan 

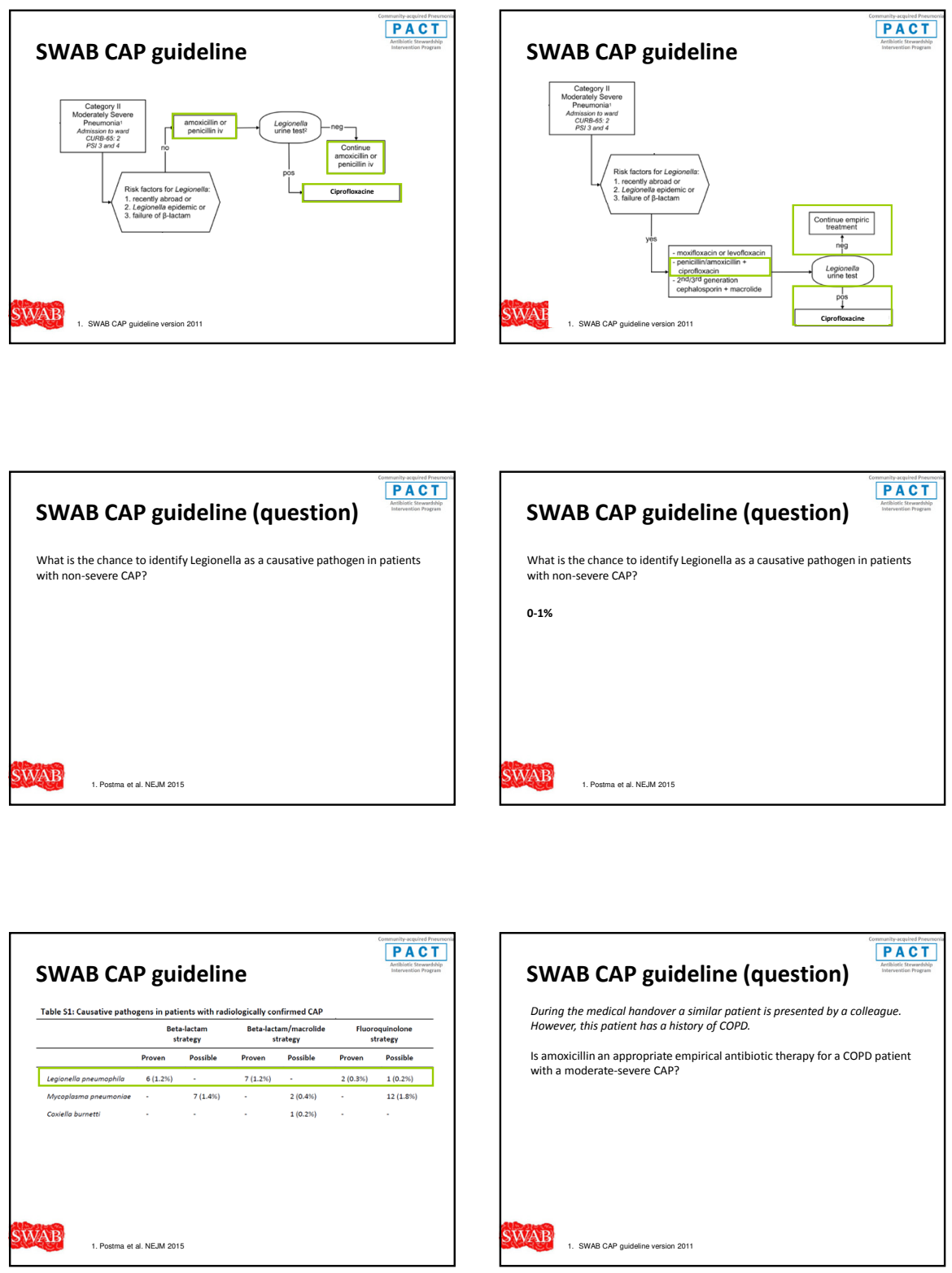

\section{SWAB CAP guideline (question)}

During the medical handover a similar patient is presented by a colleague. However, this patient has a history of COPD.

Is amoxicillin an appropriate empirical antibiotic therapy for a COPD patient with a moderate-severe CAP? 

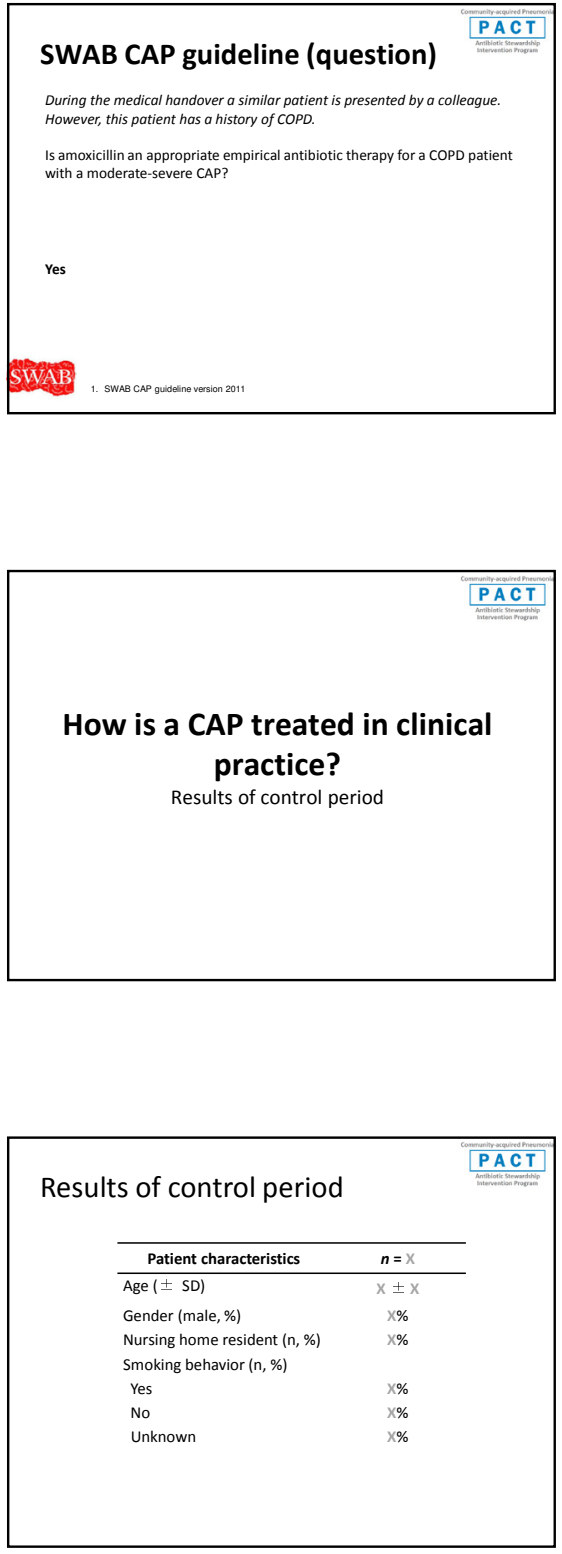

\section{SWAB CAP guideline}

PACT

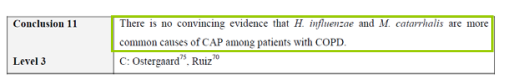

1. SWAB CAP guideline version 2011
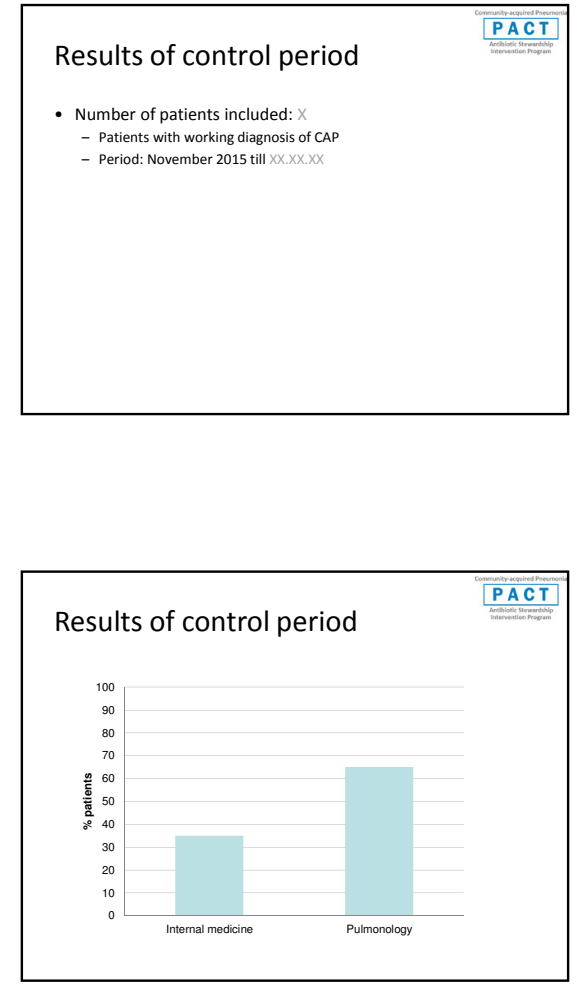


\begin{tabular}{|lc|}
\hline \multicolumn{2}{|c|}{ Results of control period } \\
\hline \multicolumn{1}{c|}{ Co-morbidities } & $n=x$ \\
\hline Heart failure & $\times \%$ \\
Cerebrovascular disease & $\times \%$ \\
Malignancy & $\times \%$ \\
Chronic liver disease & $\times \%$ \\
Chronic renal disease & $\times \%$ \\
Diabetes mellitus & $\times \%$ \\
Asthma/COPD & $\times \%$ \\
& \\
& \\
\end{tabular}

\begin{tabular}{|c|c|c|}
\hline \multicolumn{2}{|c|}{ Results of control period } & 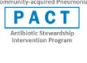 \\
\hline Antibiotic use before admission & $\boldsymbol{n}=\mathrm{X}$ & \\
\hline Amoxicillin & $\mathrm{x} \%$ & \\
\hline Amoxicillin/clavulanate & $x \%$ & \\
\hline Azithromycin & $x \%$ & \\
\hline Ciprofloxacin & $x \%$ & \\
\hline Doxycycline & $x \%$ & \\
\hline No antibiotics & $\mathrm{x} \%$ & \\
\hline
\end{tabular}
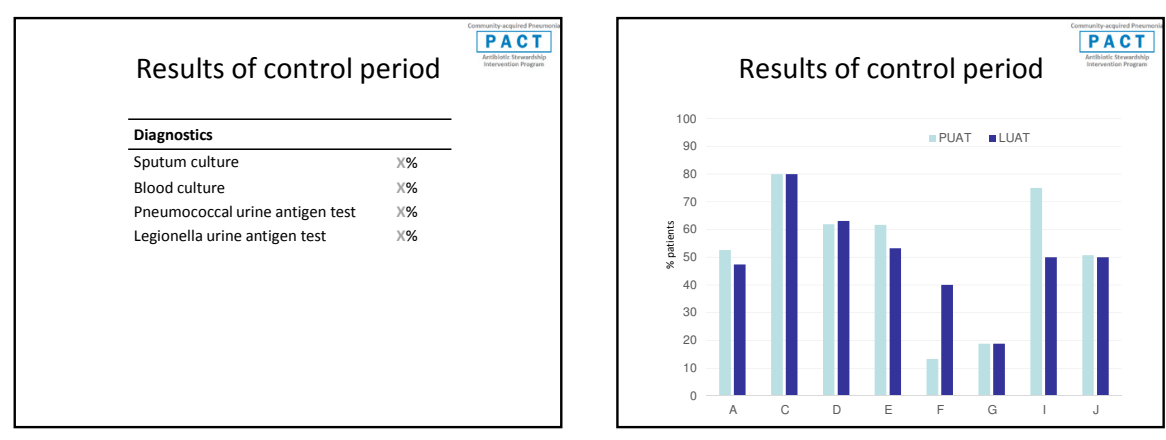

\begin{tabular}{|lc|}
\hline \multicolumn{2}{|c|}{ Results of control period } \\
\hline Empirical therapy during admission & $n=x$ \\
\hline Amoxicillin & $\mathrm{x} \%$ \\
Amoxicillin/lavulanate & $\mathrm{PAACT}$ \\
Amoxicillin/clavulanate + ciprofloxacin & $\mathrm{x} \%$ \\
Benzylpenicillin or amoxicillin + & $\mathrm{x} \%$ \\
ciprofloxacin & $\mathrm{x} \%$ \\
Ceftriaxone & $\mathrm{x} \%$ \\
Cefuroxime & $\mathrm{x} \%$ \\
Other & \\
& \\
\end{tabular}

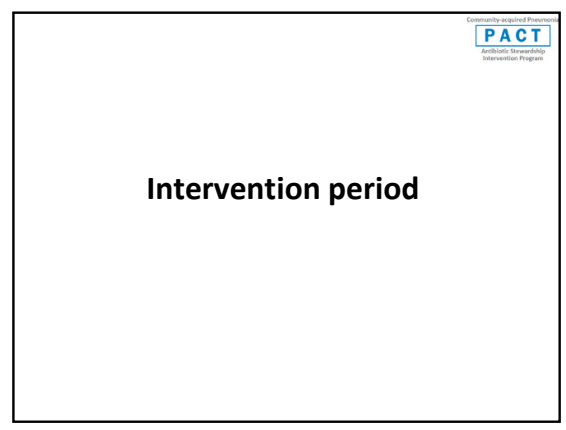




Follow-up: intervention period
- Pocket cards, posters.
- E-learning
- Daily screening and feedback from A-team

\section{Questions?}

- Researchers of CAP-PACT study:

- Inger van Heijl

- Valentijn Schweitzer

- A-team members "hospital":

$-x X X X$

$-x x x x$

$-x x x x$ 


\section{CAP-PACT study}

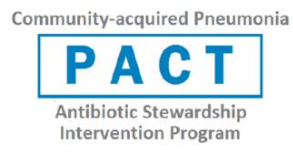

\section{Feedback form clinical lessons}

Date

\section{Content clinical lesson}

To what extent did this lesson meet your expectations?

To what extent did you acquire sufficient knowledge, insight and/or skills with this lesson?

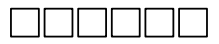

How do you rate the quality of the lesson?

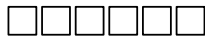

How do you rate practical usability of the lesson?

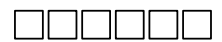

\section{Speaker:}

$\begin{array}{llllll}1 & 2 & 3 & 4 & 5 & 6\end{array}$

How do you rate his/her form of presenting?

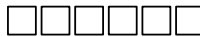

How do you rate the degree of interaction with speaker and participants?

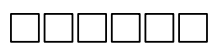

Extra comments:

What did you like?

What can be improved?

[ 1 = bad; 2 = insufficient; 3 = moderate; 4 = sufficient: 5 =good; 6 = excellent $]$ 
Appendix 4 Example of E-learning

\section{E-LEARNING COMMUNITY-ACQUIRED PNEUMONIA}

This e-learning was originally available on http://goo.gl/forms/oBbLHt3sXk made via Google Forms. This is an example of the lay-out:

\section{E-learning} Community-acquired pneumonia

Enter your details:

-Required

Hospital *

Select

Department *

Select

Position *

Select

E-mail address *

Your answer 


\section{Hospital:}

Dropdown menu with names of participating hospitals.

\section{Department:}

Dropdown menu with: Internal Medicine, Pulmonology, Emergency room, Geriatrics, Medical Microbiology.

\section{Position:}

Dropdown menu with: Medical specialist, Resident.

\section{E-mail address:}

[Open text field]

\section{Welcome:}

Welcome to the e-learning for the treatment of hospitalized patients with communityacquired pneumonia (CAP). This e-learning is an initiative from the Antimicrobial Stewardship team (A-team) to improve the treatment of hospitalized patients with CAP in your hospital.

\section{Aim:}

The aim of this e-learning is to gain inside into the treatment of CAP.

\section{Content:}

De e-learning consists of a short introduction following a number of multiple-choice questions. You can consult the corresponding literature at anytime while answering the questions. The guideline on community-acquired pneumonia is available at http://www. swab.nl/richtlijnen. After you have given your answer, you directly see whether this was correct. You will find the references of the questions at the end of the e-learning.

\section{Time:}

The total amount of time to complete the e-learning will be approximately 10 minutes. 


\section{INTRODUCTION}

Community-acquired pneumonia (CAP) is an acute infection of the lower respiratory tract which in general develops outside of a hospital. Guideline adherence is low in clinical practice. The advised empirical treatment* of CAP is based on disease-severity which is classified as mild, moderate-severe or severe that can be determined by one of the three validated scoring systems (Table 1).

When a patient is admitted with a suspected CAP the etiological cause is most often unknown. Therefore, the empirical treatment of CAP covers the most commonly isolated bacterium of CAP; Streptococcus pneumoniae. The advised antibiotic therapy for Streptococcus pneumoniae is penicillin or amoxicillin.

This e-learning is an initiative of the Antimicrobial Stewardship Team to provide more insight in the CAP guideline and thereby improve adherence in clinical practice.

*SWAB guideline: Community-acquired pneumonia (CAP), version 2011.

Table 1. three scoring systems

\begin{tabular}{lccc}
\hline & Mild CAP & Moderate- severe CAP & Severe CAP \\
\hline PSI (FINE) Score & $1-2$ & $3-4$ & 5 \\
CURB-65 (AMBU score) & $0-1$ & 2 & $3-5$ \\
Pragmatic classification & Treatment at home & Admission to a general medical ward & Admission to an Intensive Care Unit \\
\hline
\end{tabular}

\section{QUESTION 1:}

A 67-year-old man is admitted to the Pulmonology department with a working diagnosis of CAP. To start the appropriate empirical treatment, you first determine the diseaseseverity. You can choose from one of the three scoring systems; PSI score, CURB-65 score or pragmatic classification. Is there a preferred scoring system according to the guideline to determine disease-severity of CAP?

○ Yes

- No

Correct answer: No.

The guideline does not recommend any of the three scoring systems over any other. However, it is recommended that each hospital only uses one scoring system consistently in daily practice. 


\section{QUESTION 2:}

According to the pragmatic classification the CAP of the 67-year-old man is classified as moderate-severe because he is admitted to a general medical ward. Which empirical treatment is recommended for this patient?

- Azithromycin

- Ceftriaxone

- Doxycycline

- Amoxicillin or penicillin

Correct answer: Amoxicillin or penicillin.

The recommended antibiotic treatment of a moderate-severe CAP without Legionella risk factors, is amoxicillin or penicillin.

\section{QUESTION 3:}

What is the most frequently isolated causative pathogen of CAP the Netherlands?

- Haemophilus influenzae

- Mycoplasma pneumoniae

- Streptococcus pneumoniae

- Staphylococcus aureus

Correct answer: Streptococcus pneumoniae.

When a pathogen is identified, the most common isolated causative pathogen is the pneumococcus (Streptococcus pneumoniae). The empirical treatment of a patient with a non-severe CAP covers this pathogen, which is treatment with amoxicillin or penicillin. 


\section{QUESTION 4:}

What is the prevalence of penicillin-resistant Streptococcus pneumoniae in the Netherlands?
○ $0-2 \%$
○ $2-5 \%$
- $5-10 \%$
○ $>10 \%$

Correct answer: 0-2\%.

Penicillin resistance is low in the Netherlands. Amoxicillin and penicillin are effective and safe treatment options for a CAP acquired in the Netherlands.

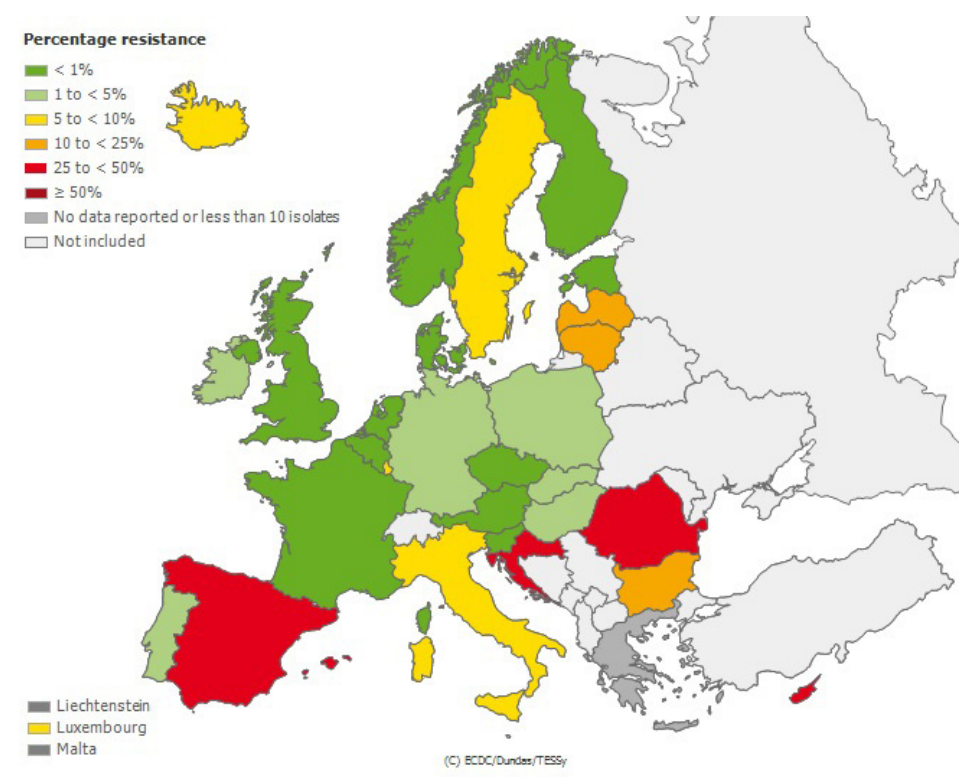

Reference: Antimicrobial resistance interactive database (EARS-Net) 2012 


\section{QUESTION 5:}

Prior to the admission at the Pulmonology department a colleague at the Emergency Room already administered one dose of ceftriaxone to the 67-year-old man. Therefore, before treatment de-escalation, you first perform the pneumococcal urine antigen test. What is the positive predictive value of this test?

\begin{tabular}{ll} 
○ & $30-50 \%$ \\
\hline & $60-70 \%$ \\
\hline & $70-80 \%$ \\
\hline & $80-100 \%$
\end{tabular}

Correct answer: 80-100\%.

The pneumococcal urine antigen test has a specificity of $96 \%$ or a positive predictive value of $88.8-96.5 \%$ (in adults). The positive predictive value (PPV) is the proportion of patients with true positive results (for the Streptococcus pneumoniae). Because of the very high PPV of the pneumococcal urine antigen test and the low resistance for penicillin, it is safe to de-escalate empirical therapy to amoxicillin or penicillin in case of a positive result.

\section{QUESTION 6:}

The turnaround time of a pneumococcal urine antigen test is 15 minutes. One hour after you performed this test, you phone the Microbiology department. The test result is negative. In the meantime, your 67-year-old patient does not improve after 1 day of treatment with ceftriaxone. You consider adding ciprofloxacin to the initial therapy to cover a possible Legionella infection. In which situation is it appropriate to add antibiotic treatment for Legionella infection?

- Age $\geq 65$ years

- History of COPD

- History of obstructive pulmonary disease

- Failure to improve despite $\geq 48$ hours treatment with a beta-lactam antibiotic at adequate dosage.

Correct answer: Failure to improve despite $\geq 48$ hours treatment with a beta-lactam antibiotic at adequate dosage.

It is appropriate to add Legionella coverage in case of no clinical improvement after 48 hours of treatment with beta-lactam antibiotics in correct dosages. With a urine antigen test a Legionella infection can be determined. Risk factors for Legionella infection are: 
(1) recent visit to a foreign country, (2) coming from an epidemic setting of Legionella spp. infections, (3) failure to improve despite $\geq 48$ hours treatment with a beta-lactam antibiotic at adequate dosage.

\section{QUESTION 7:}

What is the chance to identify Legionella as a causative pathogen in patients with nonsevere CAP?

$$
\begin{array}{cc}
\circ & 0-1 \% \\
\hline & 1-2 \% \\
\hline & 2-5 \% \\
\hline & 5-10 \%
\end{array}
$$

Correct answer: 0-1\%.

In a recent study performed in 7 Dutch hospitals, Legionella was identified in $0.7 \%$ of the patients that were admitted with CAP to a general medical ward.

\section{QUESTION 8:}

During the medical handover a similar patient is presented by a colleague. However, this patient has a history of COPD. Is amoxicillin an appropriate empirical antibiotic therapy for a COPD patient with a moderate-severe CAP?

$$
\begin{array}{ll}
\circ & \text { Yes } \\
\circ & \text { No }
\end{array}
$$

Correct answer: Yes.

Amoxicillin is an appropriate empirical antibiotic therapy for a COPD patient with a moderate-severe CAP. There is no evidence that a CAP in COPD patients is caused by other pathogens.

\section{REFERENCES}

\begin{tabular}{|l|l|}
\hline $\begin{array}{l}\text { Introduction, } \\
\text { Question 1-3, 6-8. }\end{array}$ & SWAB/NVALT guideline Community-acquired Pneumonia, November 2011 \\
\hline Question 4 & European Antimicrobial Resistance Surveillance (EARSS), report 2012 \\
\hline Question 5 & $\begin{array}{l}\text { Sinclair A et al. Systematic review and meta-analysis of a urine-based pneumococcal antigen test for } \\
\text { diagnosis of community-acquired pneumonia caused by Streptococcus pneumoniae. J Clin Microbiol. } \\
\text { 2013;51(7):2303-10. }\end{array}$ \\
& $\begin{array}{l}\text { Sordé R, et al. Current and potential usefulness of pneumococcal urinary antigen detection in } \\
\text { hospitalized patients with community-acquired pneumonia to guide antimicrobial therapy. Arch Intern } \\
\text { Med. 2011:171(2):166-72. }\end{array}$ \\
\hline Question 8 & $\begin{array}{l}\text { Postma DF et al. Antibiotic treatment strategies for community-acquired pneumonia in adults. N. Eng J. } \\
\text { Med 2015;372(14):1312-23. }\end{array}$ \\
\hline
\end{tabular}




\section{FINISHED!}

Thank you for completing the e-learning.

Your hospital is participating in the CAP-PACT study and this e-learning is part of the study. The aim of the CAP-PACT study is to increase the knowledge of the CAP guideline by means of an e-learning and clinical lessons. And thereby decrease the unnecessary use of broad-spectrum antibiotics in patients with CAP.

If you have any questions please contact your local antimicrobial stewardship team or consult your local antibiotic formulary.

Please do not forget to click on the 'SEND' button at the bottom of this page. 


\section{CAP-PACT study}

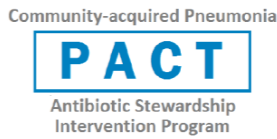

\section{Community-acquired pneumonia}

\section{Pneumonia acquired outside the hospital}

- No recent hospital admission $>48 \mathrm{hr}$ ( $<2$ weeks)

- No recent residence in long-term care facility $>48 \mathrm{hr}$ ( $<2$ weeks)

\section{Classification}

\begin{tabular}{l|l|l|l} 
Disease severity & \multicolumn{2}{l}{ Classification } & PSI score \\
\hline \hline & CURB-65 & Pragmatic score & $1-2$ \\
\hline Mild CAP & $0-1$ & No admission & $3-4$ \\
\hline $\begin{array}{l}\text { Moderate-severe } \\
\text { CAP }\end{array}$ & 2 & $\begin{array}{l}\text { Admission at a non-ICU } \\
\text { ward }\end{array}$ & ICU admission \\
\hline Severe CAP & $3-5$ & ICU
\end{tabular}

\section{Diagnostics}

Blood culture, sputum culture and pneumococcal urinary antigen test Legionella urinary antigen test only in patients with a severe CAP or with risk factors for Legionnaires disease. Risk factors:

- Travel abroad

- Legionnaires disease outbreak

- No effect of penicillins or cephalosporins after 48uur

\section{Empirical therapy}

\begin{tabular}{l|l|l}
\multicolumn{1}{l|}{ Disease severity } & Antibiotics & Route \\
\hline $\begin{array}{c}\text { Mild CAP } \\
1^{\text {st }} \text { line } \\
2^{\text {nd }} \text { line }\end{array}$ & Amoxicillin \\
$\begin{array}{c}\text { Moderate-severe CAP } \\
1^{\text {st }} \text { line }\end{array}$ & Oral \\
Ornstige CAP \\
$\begin{array}{l}\text { Combination therapy } \\
\text { Amoxicillin }\end{array}$ & IV/oral \\
& Benzylpenicillin + ciprofloxacin & IV
\end{tabular}


Appendix 6 Example of poster

\section{Patient with moderate-severe CAP}

\section{Empirical therapy}
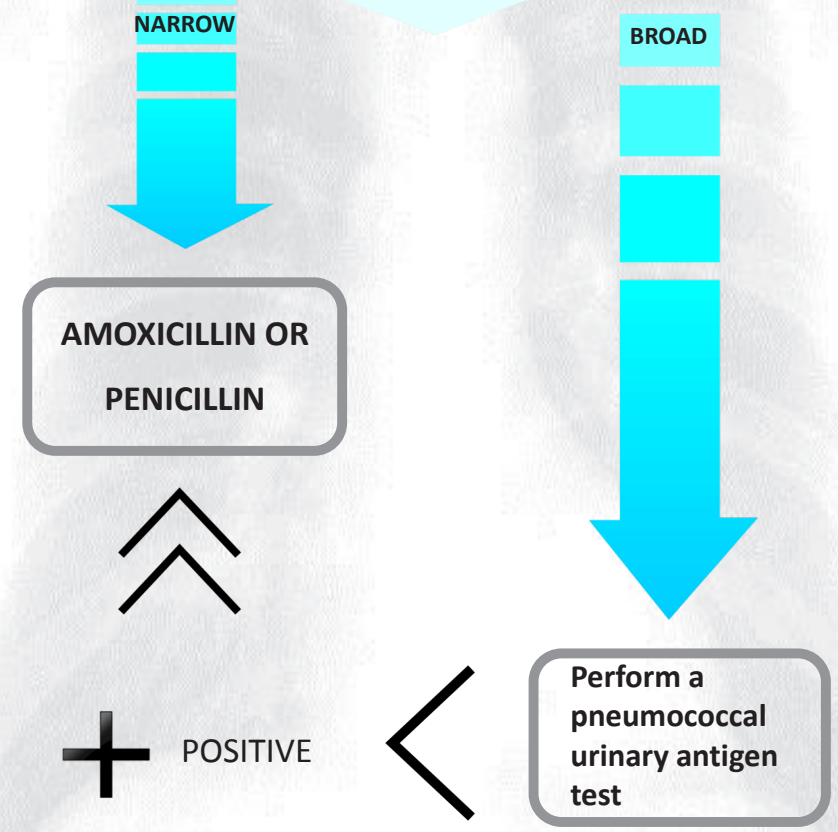



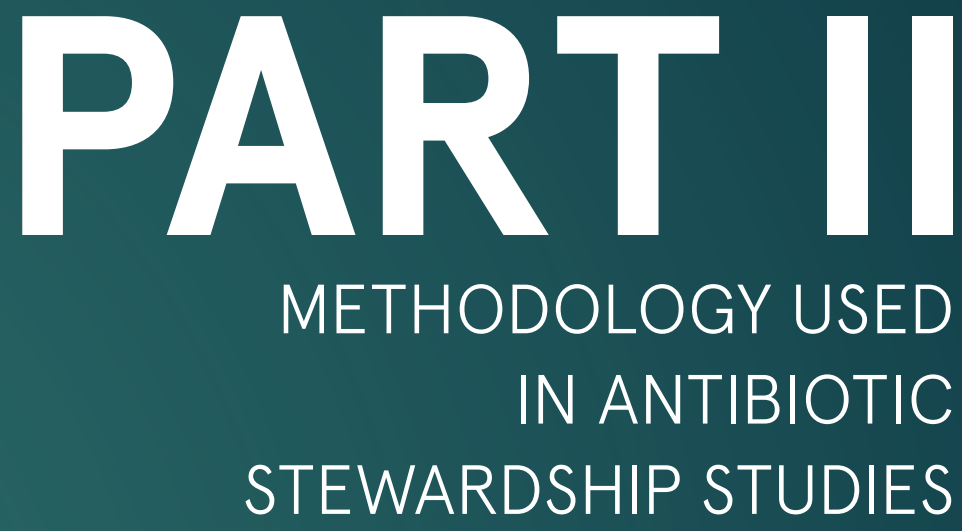


abo

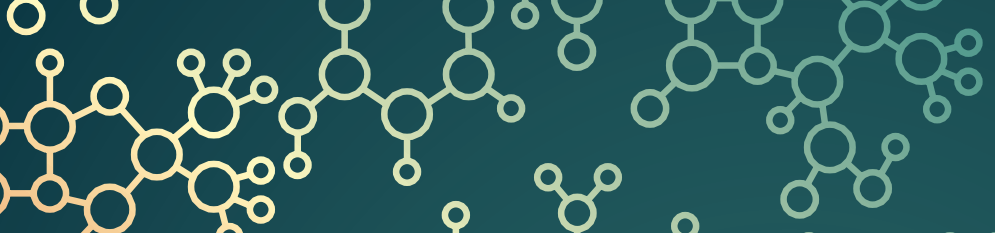
o

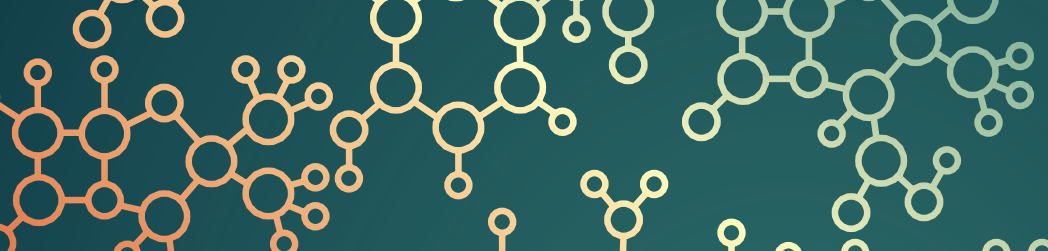

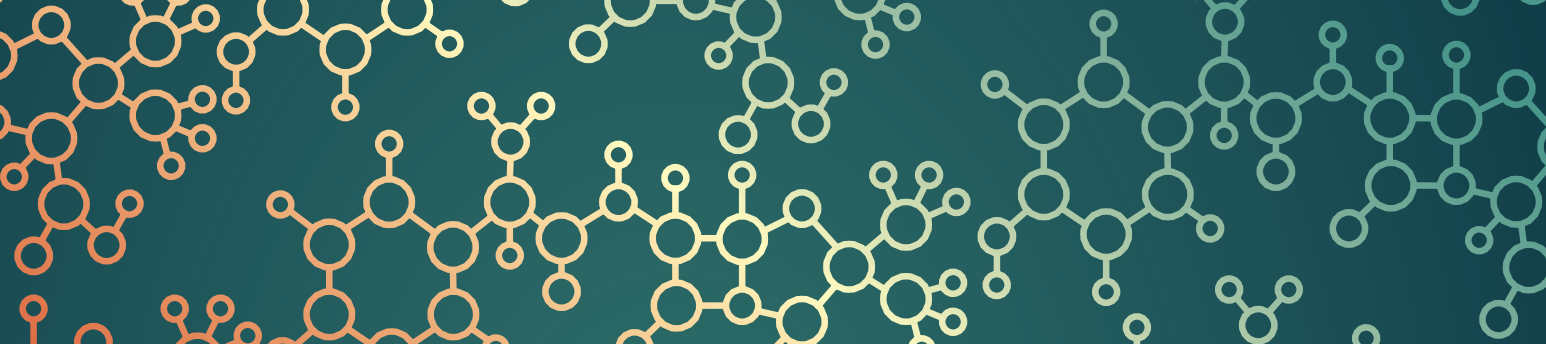

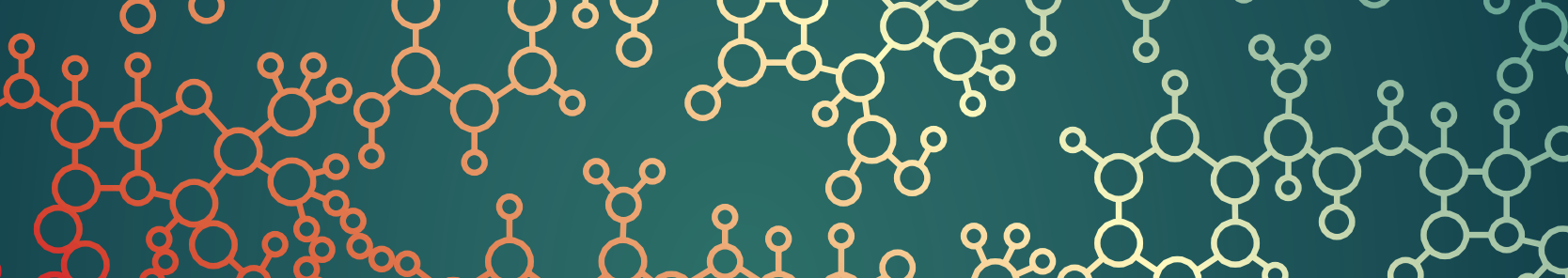
80\%

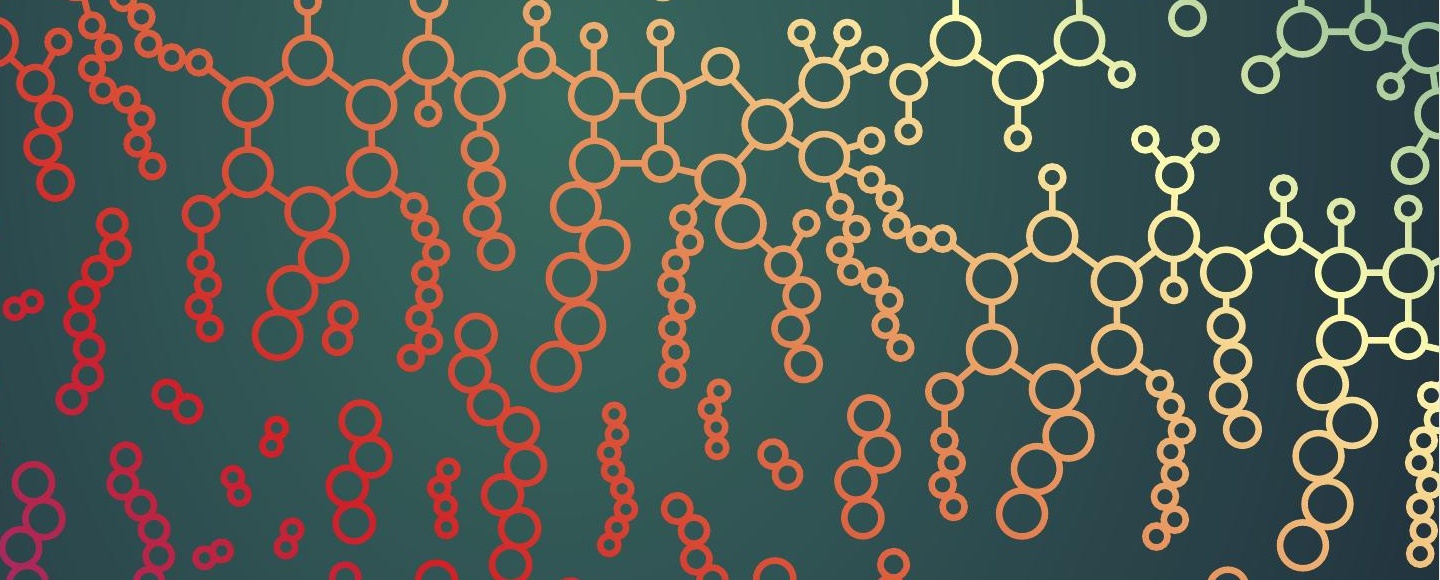
$88 \% 888$ $\circ 88$ \& 8

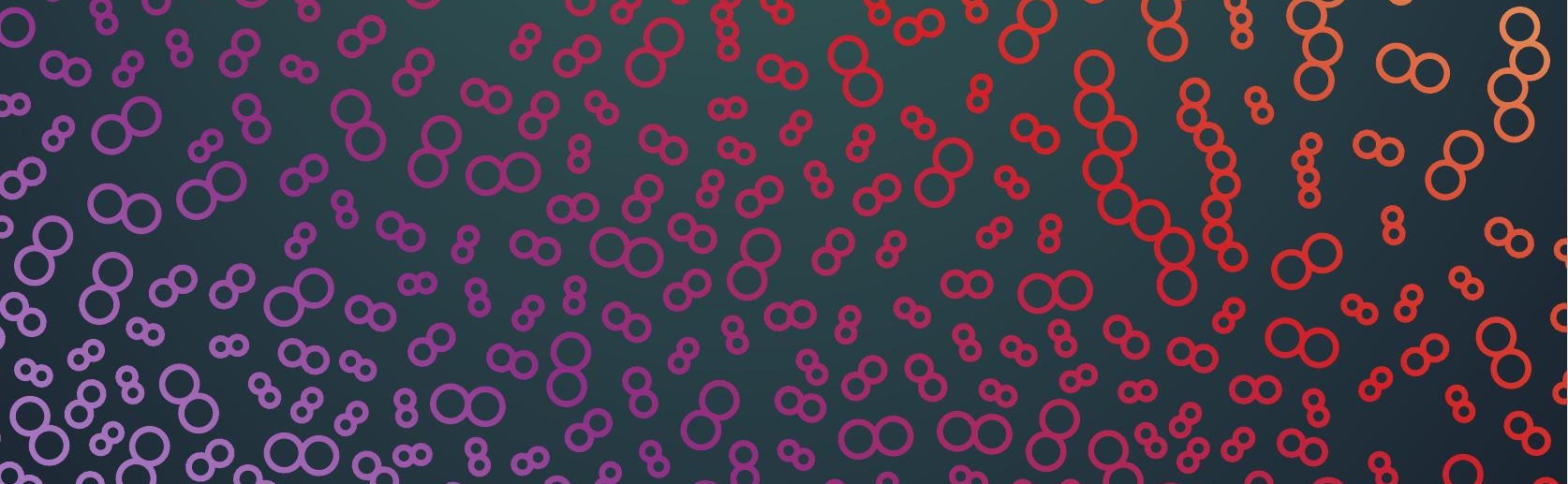




\section{The quality of studies evaluating antimicrobial stewardship interventions: a systematic review.}

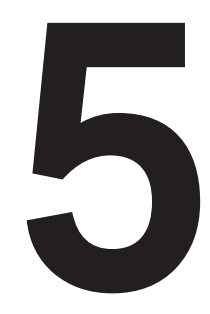

Valentijn A. Schweitzer, Inger van Heijl, Cornelis H. van Werkhoven, Jasmin Islam, Kelly D. Hendriks-Spoor, Julia Bielicki, Marc J.M. Bonten, A. Sarah Walker, Martin J. Llewelyn, consensus on Antimicrobial Stewardship Interventions (CASE) Study Group 


\section{ABSTRACT}

\section{Background}

Antimicrobial stewardship aims to optimize antibiotic use and minimize selection of antimicrobial resistance. The methodological quality of published studies in this field is unknown.

\section{Aims}

Our objective was to perform a comprehensive systematic review of antimicrobial stewardship research design and identify features which limit validity and translation of research findings into clinical practice.

\section{Sources}

The following online database was searched: PubMed.

\section{Study eligibility criteria}

Studies published between January 1950 and January 2017, evaluating any antimicrobial stewardship intervention in the community or hospital setting, without restriction on study design or outcome.

\section{Methods}

We extracted data on pre-specified design quality features and factors that may influence design choices including (1) clinical setting, (2) age group studied, (3) when the study was conducted, (4) geographical region, and (5) financial support received.

\section{Results}

The initial search yielded 17382 articles; 1008 were selected for full-text screening, of which 825 were included. Most studies (675/825, 82\%) were non-experimental; 104 (15\%) used interrupted time series analysis, 41 (6\%) used external controls, and 19 (3\%) used both. Studies in the community setting fulfilled a median of five out of 10 quality features (IQR 3-7) and 3 (IQR 2-4) in the hospital setting. Community setting studies (25\%, 205/825) were significantly more likely to use randomization (OR 5.9; 95\% Cl 3.8-9.2), external controls (OR 5.6; $95 \% \mathrm{Cl} 3.6-8.5)$, and multiple centres $(\mathrm{OR} 10.5 ; 95 \% \mathrm{Cl} 7.1-$ 15.7). From all studies, only $48 \%$ (398/825) reported clinical and $23 \%$ (190/825) reported microbiological outcomes. Quality did not improve over time.

\section{Conclusions}

Overall quality of antimicrobial stewardship studies is low and has not improved over time. 
Most studies do not report clinical and microbiological outcome data. Studies conducted in the community setting were associated with better quality. These limitations should inform the design of future stewardship evaluations so that a robust evidence base can be built to guide clinical practice.

\section{INTRODUCTION}

Antimicrobial resistance (AMR) is increasing globally and is a substantial threat to human health ${ }^{[1]}$. There is a clear relationship between antibiotic exposure and AMR both in populations ${ }^{[2]}$ and individual patients ${ }^{[3]}$. An estimated $30 \%$ of human antibiotic use may be unnecessary and healthcare systems around the world are aiming to achieve substantial reductions in unnecessary antibiotic prescribing. The term antimicrobial stewardship' is used to describe use of antibiotics which balances the need for effective individual treatment against the longer-term, societal impact of antibiotic use on antibiotic resistance ${ }^{[4]}$. Interventions to improve antimicrobial stewardship are usually multifaceted and include periodic or individual patient audit and feedback, decision support, education (educational meetings, educational materials), and antimicrobial formulary restriction ${ }^{[5]}$.

Recognition of the threat posed by AMR and the need to optimize antibiotic prescribing has driven an exponential increase in the publication of studies evaluating antimicrobial stewardship interventions over the last 20 years ${ }^{[6]}$. Previous systematic reviews have synthesized this evidence with the aim of making recommendations for practice ${ }^{[5,7-12]}$. These have, appropriately, considered studies with the lowest possible risk of bias but have excluded $>50 \%$ of published studies in which methodological quality falls below the Effective Practice and Organization of Care (EPOC) criteria ${ }^{[13]}$. Because the minority of studies are of sufficient quality, many areas of practice rely on a weak evidence base ${ }^{[8]}$; conducting studies which do not inform practice is a waste of time and valuable resources ${ }^{[5,12]}$.

Journals are beginning to report the minimum standards for antimicrobial stewardship studies to be published ${ }^{[14]}$; however, there remains a need for consensus on how to design, analyse, and report studies evaluating interventions to improve antibiotic prescribing. This would optimize use of valuable resources and strengthen the evidence base in this field. Currently, no overview exists of how antimicrobial stewardship evaluations are designed. We conducted a systematic review of antimicrobial stewardship evaluations with the aim 
of identifying areas in stewardship evaluation most in need of improvement, to increase validity and translation of research findings into clinical practice.

\section{METHODS}

\section{Search strategy}

We searched PubMed for studies evaluating antimicrobial stewardship interventions between January 1950 and January 2017. The search strategy (Appendix 1) was designed to be as broad as possible. Inclusion criteria were any study evaluating an antimicrobial stewardship intervention, without restriction on the type of intervention studied and what outcomes were evaluated. Studies were excluded if they were (1) not in English, (2) case reports, (3) focused mainly on HIV, or (4) narrative or systematic reviews. All studies were screened by one author (V.A.S., C.H.v.W., J.I., K.H., or I.V.H.). In case of duplicate publications, only the original article was included. A random selection of $700(\sim 4 \%$ of total) studies was assessed by a second author (V.A.S., C.H.v.W., J.I., K.H., or I.v.H.). Uncertainties about the inclusion of studies was resolved by discussion.

\section{Data extraction}

Studies fulfilling the inclusion criteria were identified by screening on title and abstract. All selected studies then underwent full-text evaluation by one author (V.A.S., C.H.v.W., J.I., K.H., or I.V.H.) against the inclusion and exclusion criteria and data were subsequently extracted using a standard data extraction template (Appendix 2). Data were extracted on study characteristics (i.e. title, authors, year of publication), design quality features, and factors possibly associated with methodological quality. If no funding was reported it was assumed that studies received no financial support. Authors were not contacted in case data were missing or incomplete. A random selection of $10 \%$ of the studies was extracted by a second author. We followed the PRISMA criteria for the reporting of systematic reviews ${ }^{[15]}$.

\section{Selection of quality features, and factors associated with quality}

In February 2017 we established an international Consensus Working Group funded by the Joint Programming Initiative on Antimicrobial Resistance (JPIAMRWG-010) to develop recommendations on the design, analysis, and reporting of antimicrobial stewardship evaluations. The working group coordinators (V.A.S., C.H.v.W., M.L., A.S.W., M.B.) invited members to the consensus group based on their expertise on antimicrobial stewardship and/or trial methodology, ensuring that all key clinical areas (primary care, secondary care, intensive care medicine, and paediatrics) were represented. The consensus 
group agreed that a review to identify areas in stewardship evaluation most in need of improvement to increase validity and translation of research findings into clinical practice was required. For this purpose, existing quality scores were not applicable because these focus solely on methodological quality. The group selected quality features and factors likely to underlie design quality and features based on plenary group discussion during the consensus meeting. Ten quality features were selected for assessment: (1) randomized research design used, (2) external control group assessed, (3) multiple centres used, (4) sustainability of the intervention sufficiently assessed ( $\geq 12$ months), (5) sample size calculation reported, (6) prospective data collection, (7) correction for confounding factors, (8) primary outcome defined and reported, (9) clinical outcome reported, and (10) microbiological outcome reported. Selected factors likely to underlie design quality features were (a) the clinical setting (community versus hospital), (b) age group studied (studies including children versus adults, or both), (c) year when study was conducted (newer versus older studies, categorized at approximate quintiles: 1977-2004, 2005-2010, 2011-2013, 2014-2015, 2016-2017), (d) geographical region, and (e) financial support. The quality features and corresponding categorizations are shown in Table S1.

\section{Statistical analysis}

Descriptive statistics were used to describe the quality features of included studies. Differences stratified by subgroup were displayed using spider graphs (Microsoft Excel, version 2010). To assess the independent relationship between factors and quality features, we performed multivariable logistic regression models with backward stepwise selection (exit $p>0.10$ ), presenting odds ratios and $95 \%$ confidence intervals in a heat map displaying the strength of the association (Microsoft Excel, version 2010). Analyses were performed using the Statistical Package for the Social Sciences for Windows (Version SPSS 21.0).

\section{RESULTS}

The initial search yielded 17,382 articles. After title and abstract screening, 1,008 articles were selected for full-text screening (Figure 1). Of these, 183 were excluded, leaving 825 articles for full assessment (Appendix 3). Among 700 randomly selected articles that were screened by a second author, 640 out of 700 (91\%) were excluded by both authors, 23 out of $700(3.3 \%)$ were selected for inclusion by both authors, 13 out of 700 (1.8\%) were selected for inclusion by only the first author, and 24 out of 700 (3.4\%) were selected for inclusion by only the second author, resulting in a percentage agreement after title/ abstract screening of $95 \%$, with a moderate interobserver agreement (Cohen's kappa, 
0.53). After discussion of the full text articles, consensus about inclusion was reached in $99.5 \%(696 / 700)$ of the selected articles. From the $3.4 \%(24 / 700)$ studies that were newly identified by a second author, 7/700 (1.0\%) were considered correct inclusions after discussion. Therefore, the low proportion of missed papers justified not screening in duplicate. Among the 83 articles selected for double data extraction by a second author, the percentage agreement per variable ranged from $91 \%$ to $100 \%$, with all the quality features showing an agreement of $\geq 95 \%$.

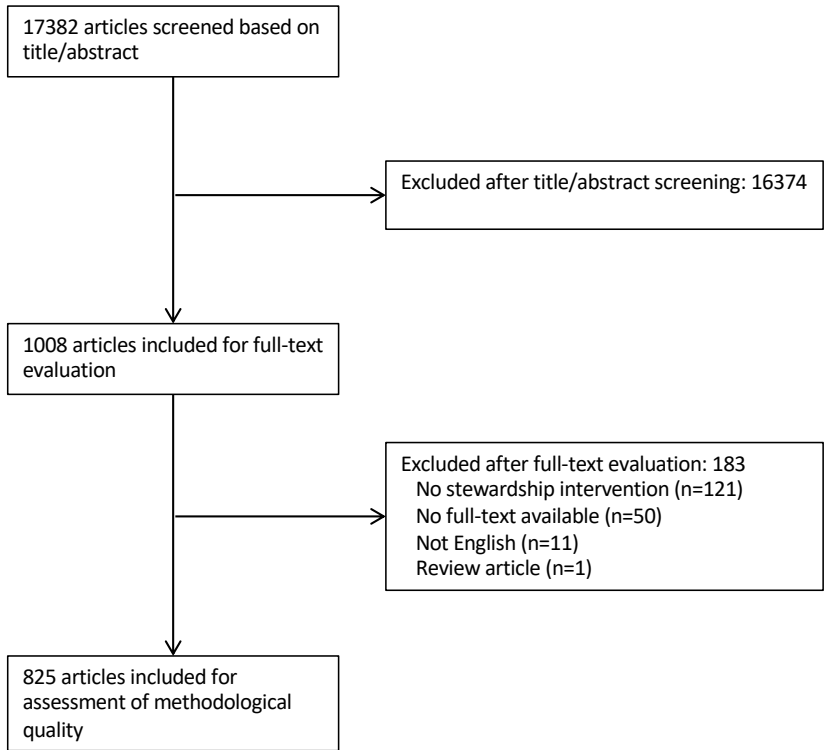

Figure 1 Flowchart of the studies included in the systematic literature review.

\section{Characteristics of included studies}

A total of 825 studies were included. In the community setting, most studies were multicentre $(72 \%, 148 / 205)$ and the commonest study designs were before-after studies without an interrupted time series (ITS) analysis (23\%, 48/205), randomized controlled trials (14\%, 28/205), and parallel cluster randomized trials (15\%, 30/205) (Table 1). Among the included studies in the hospital setting, most were single centre $(84 \%, 519 / 620)$ and the commonest study designs were before-after studies without an ITS analysis (59\%, 365/620), before-after studies with an ITS analysis (13\%,82/620), and cohort studies without a control group $(12 \%, 75 / 620)$. Among the before-after studies without an ITS analysis, the majority were single centre (86\%, 352/411), and retrospective (58\%, 239/411). 
In the $86(10 \%)$ cluster randomized studies a median of 28 clusters were randomized, with $57 \%$ (49/86) randomizing $\geq 20$ clusters, $28 \%$ (24/86) randomizing $<15$ clusters, $21 \%$ $(18 / 86)$ randomizing $<10$ clusters, and $9 \%(8 / 86)$ randomizing $<5$ clusters. In the hospital setting, $13 \%(2 / 13)$ randomized $\geq 20$ clusters, while in the community setting $65 \%(64 / 71)$ randomized $\geq 20$ clusters. A minority of studies were conducted in children (12\%, 101/825). Both in the community and the hospital setting, most studies did not target a specific disease or syndrome $(31 \%, 63 / 205,55 \% 324 / 620$, respectively) or specific antibiotic class (86\%, 177/205, 69\% 427/620, respectively). The majority of interventions were bundles $(57 \%, 470 / 825)$. Commonest interventions in the community setting included education $(73 \%, 149 / 205)$, audit with periodic feedback (29\%,59/205), and clinical decision support $15 \%(31 / 205)$. In the hospital setting, commonest interventions included education $42 \%$ (260/620), audit and feedback on an individual patient level (40\%,245/620), restriction $(18 \%, 113 / 620)$, and clinical decision support $(18 \%, 112 / 620)$. Both in the community and in the hospital setting, virtually all included studies reported process measure outcomes (99\%, 818/825) (Table 2). Both in the community setting and in the hospital the most commonly reported process measures included the proportion of patients treated with antibiotics (59\%, 121/205, 21\%, 131/620), costs/cost-effectiveness (18\%, 36/205, 32\%, 200/620, respectively), appropriateness (17\%, 34/205, 29\%, 178/620, respectively), and defined daily doses $(17 \%, 34 / 205,25 \%, 156 / 620$, respectively). In the community setting, commonest reported clinical outcomes were revisits $(11 \%, 22 / 205)$, clinical cure $(6 \%, 12 / 205)$, and infection $(5 \%, 10 / 205)$, while in the hospital setting these were mortality $(32 \%, 302 / 620)$, length of stay $(32 \%, 201 / 620)$, and hospital readmissions $(12 \%, 76 / 620)$.

Table 1 Characteristics of included studies stratified by studies performed in the community and the hospital setting

\begin{tabular}{|c|c|c|}
\hline & Community $(n=205)$ & Hospital $(n=620)$ \\
\hline Study characteristics & $n(\%)$ & $\mathrm{n}(\%)$ \\
\hline Number of patients included (median, IQR) & $1255(278-11230)$ & $423(186-1398)$ \\
\hline Number of centres involved (median, IQR) & $27(8-90)$ & $1(1-1)$ \\
\hline \multicolumn{3}{|l|}{ Age } \\
\hline Adults & $64(31)$ & $285(46)$ \\
\hline Children & $33(16)$ & $68(11)$ \\
\hline Both & $108(53)$ & $267(43)$ \\
\hline \multicolumn{3}{|l|}{ Specific disease targeted } \\
\hline No specific disease targeted & $63(31)$ & $342(55)$ \\
\hline Upper respiratory tract infections & $106(52)$ & $25(4)$ \\
\hline Lower respiratory tract infections & $57(28)$ & $70(11)$ \\
\hline Bacteraemia & $0(0)$ & $42(7)$ \\
\hline Urinary tract infections & $18(9)$ & $27(4)$ \\
\hline Prophylaxis & $2(1)$ & $39(6)$ \\
\hline Sepsis & $0(0)$ & $17(3)$ \\
\hline Skin and soft tissue infections & $1(1)$ & $8(1)$ \\
\hline Abdominal infections & $3(1)$ & $10(2)$ \\
\hline Other & $16(8)$ & $62(10)$ \\
\hline
\end{tabular}


Table 1 continued.

\begin{tabular}{|c|c|c|}
\hline Study characteristics & $\begin{array}{l}\text { Community }(n=205) \\
n(\%)\end{array}$ & $\begin{array}{l}\text { Hospital }(n=620) \\
n(\%)\end{array}$ \\
\hline \multicolumn{3}{|l|}{ Antibiotic class targeted } \\
\hline No specific antibiotic class targeted & $177(86)$ & $427(69)$ \\
\hline Cephalosporins & $4(2)$ & $66(11)$ \\
\hline Fluoroquinolones & $10(5)$ & $54(9)$ \\
\hline Carbapenems & $0(0)$ & $48(8)$ \\
\hline Vancomycin & $0(0)$ & $31(5)$ \\
\hline Aminoglycosides & $0(0)$ & $28(5)$ \\
\hline Penicillins & $10(5)$ & $15(2)$ \\
\hline Macrolides & $8(4)$ & $5(1)$ \\
\hline Other & $17(8)$ & $89(14)$ \\
\hline \multicolumn{3}{|l|}{ Antimicrobial stewardship interventions } \\
\hline Education & 149 (73) & $260(42)$ \\
\hline Audit and feedback - individual patient & $12(6)$ & $245(40)$ \\
\hline Audit and feedback - periodic & $59(29)$ & $82(13)$ \\
\hline Restriction & $13(6)$ & $113(18)$ \\
\hline Clinical decision support & $31(15)$ & $112(18)$ \\
\hline Rapid diagnostic testing & $24(12)$ & $68(11)$ \\
\hline Therapeutic drug monitoring & $0(0)$ & $15(2)$ \\
\hline Guideline implementation & $20(10)$ & $95(15)$ \\
\hline Delayed prescribing & $9(4)$ & $0(0)$ \\
\hline Other & $42(20)$ & $78(13)$ \\
\hline \multicolumn{3}{|l|}{ Research designs } \\
\hline Before-after study & $48(23)$ & $365(59)$ \\
\hline Before-after study (ITS*) & $22(11)$ & $82(13)$ \\
\hline Cohort without control group & $6(3)$ & $75(12)$ \\
\hline Controlled before-after study & $22(11)$ & $19(3)$ \\
\hline Randomised controlled trial (RCT) & $28(14)$ & $40(6)$ \\
\hline Parallel cluster randomised trial & $30(15)$ & $6(1)$ \\
\hline Parallel cluster randomised trial with baseline period & $26(13)$ & $2(1)$ \\
\hline Controlled before-after study (ITS) & $7(3)$ & $12(2)$ \\
\hline Non-randomised parallel cluster study & $5(2)$ & $8(1)$ \\
\hline Factorial cluster randomised trial & $7(3)$ & $0(0)$ \\
\hline Cluster randomised cross-over trial & $2(1)$ & $3(1)$ \\
\hline Stepped wedge cluster randomised trial & $0(0)$ & $1(1)$ \\
\hline Non-randomised cluster cross-over study & $0(0)$ & $3(1)$ \\
\hline Factorial randomised controlled trial (RCT) & $1(1)$ & $2(1)$ \\
\hline Non-randomised stepped wedge study & $0(0)$ & $2(1)$ \\
\hline Adaptive RCT & $1(1)$ & $0(0)$ \\
\hline
\end{tabular}

IQR: interquartile range, *ITS: interrupted time series

\section{Quality features}

The percentage of studies including each quality feature is shown in Table 3. Studies in the community setting fulfilled a median of five quality features (IQR 3-7), while studies in the hospital setting fulfilled three (IQR 2-4). None fulfilled all 10 quality features. In the community setting $2 \%(4 / 205)$ fulfilled nine, $16 \%$ (33/205) fulfilled at least eight, and $35 \%(72 / 205)$ fulfilled at least seven quality indicators. In the hospital setting $1 \%(4 / 620)$ fulfilled nine, 3\% (19/620) fulfilled eight, and 6\% (37/620) fulfilled seven quality indicators. Of note, there were substantial differences between studies which did and did not use randomized designs to the extent to which other quality features were present. Among the 150 randomized studies, all used an external control group, 71\% (107/150) included multiple centres, 64\% (97/150) reported a sample size calculation and 96\% (144/150) collected 
data prospectively. In contrast, among the non-randomized studies, $12 \%$ (78/675) used an external control group, 21\% (142/675) included multiple centres, 11\% (76/675) reported a sample size calculation and $43 \%(288 / 675)$ collected data prospectively.

Table 2 Outcomes reported in the included antimicrobial stewardship studies stratified by studies performed in the community and the hospital setting

\begin{tabular}{|c|c|c|}
\hline & $\begin{array}{l}\text { Community ( } n=205) \\
n(\%)\end{array}$ & $\begin{array}{l}\text { Hospital }(n=620) \\
n(\%)\end{array}$ \\
\hline \multicolumn{3}{|l|}{ Process measure outcomes } \\
\hline Costs/cost-effectiveness & $36(18)$ & $200(32)$ \\
\hline Appropriateness & $34(17)$ & $178(29)$ \\
\hline Defined daily doses (DDD) & $34(17)$ & $156(25)$ \\
\hline Proportion treated with antibiotics & $121(59)$ & $131(21)$ \\
\hline Recommendation acceptance & $6(3)$ & $114(18)$ \\
\hline Guideline adherence & $27(13)$ & $100(16)$ \\
\hline Duration of treatment & $5(2)$ & $93(15)$ \\
\hline Days on therapy (DOT) & $6(3)$ & $62(10)$ \\
\hline Time to appropriate therapy & $3(1)$ & $71(11)$ \\
\hline Antibiotic knowledge & $17(8)$ & $14(2)$ \\
\hline None & $2(1)$ & $5(1)$ \\
\hline Other & $58(28)$ & $166(27)$ \\
\hline \multicolumn{3}{|l|}{ Clinical outcome measures } \\
\hline None & $144(70)$ & $283(46)$ \\
\hline Mortality & $9(4)$ & $203(33)$ \\
\hline Length of stay & $5(2)$ & $201(32)$ \\
\hline Infection & $10(5)$ & $75(12)$ \\
\hline Hospital readmission & $0(0)$ & $76(12)$ \\
\hline Adverse effects & $5(2)$ & $52(8)$ \\
\hline Revisits & $22(11)$ & $0(0)$ \\
\hline Clinical cure & $12(6)$ & $27(4)$ \\
\hline Intensive care unit admission & $0(0)$ & $24(4)$ \\
\hline Hospital admission & $9(4)$ & $0(0)$ \\
\hline Time to clinical stability & $3(1)$ & $6(1)$ \\
\hline Other & $16(8)$ & $49(8)$ \\
\hline \multicolumn{3}{|l|}{ Microbiological outcome measures } \\
\hline None & $188(92)$ & $447(72)$ \\
\hline Colonization/infection resistant pathogens & $17(8)$ & $146(24)$ \\
\hline Clostridium difficile infections & $5(2)$ & $62(10)$ \\
\hline Other & $2(1)$ & $12(2)$ \\
\hline
\end{tabular}

Table 3 Design quality features of the included studies stratified by studies performed in the community and the hospital setting

\begin{tabular}{lll}
\hline Quality feature & Community (n=205) & Hospital (n=620) \\
& $\mathbf{n}(\%)$ & $\mathbf{n}(\%)$ \\
\hline Randomised research design & $95(46)$ & $55(9)$ \\
External control group & $129(63)$ & $99(16)$ \\
Multicentre & $148(72)$ & $101(16)$ \\
Sample size calculation reported & $77(38)$ & $96(15)$ \\
Prospective data collection & $144(70)$ & $288(46)$ \\
Correction for confounding factors & $113(55)$ & $157(25)$ \\
Primary outcome defined & $116(57)$ & $272(44)$ \\
Clinical outcome reported & $61(30)$ & $337(54)$ \\
Microbiological outcome reported & $17(8)$ & $173(28)$ \\
Sustainability assessed ( $\geq 12$ months) & $115(56)$ & $347(56)$ \\
\hline
\end{tabular}




\section{Factors associated with design quality}

Design quality was considerably better in almost all quality aspects of community versus hospital setting studies (Figure S1A), with more use of randomized designs (46\% vs. 9\%), external controls (63\% vs. $16 \%$ ), sample size calculations (38\% vs. $15 \%$ ), prospective data collection (70\% vs. $46 \%$ ), correction for confounding (55\% vs. $25 \%$ ), use of a defined primary outcome (57\% vs. $44 \%$ ), and involvement of multiple centres ( $72 \%$ vs. $16 \%$ ). However, community setting studies less often reported clinical (30\% vs. 54\%) and microbiological ( $8 \%$ vs. $28 \%$ ) outcomes. Community setting remained significantly associated with all these factors in multivariable models (Table 4). From the studies that reported financial support, 20\% (53/264) were industry funded, and 84\% (221/264) were publicly funded. Studies with financial support were of higher methodological quality than studies without (Figure S1B), as they more frequently used randomized designs (31\% vs. $7 \%$ ), external controls ( $34 \%$ vs. $8 \%$ ), sample size calculations (33\% vs. $11 \%$ ), prospective data collection (63\% vs $43 \%$ ), correction for confounding ( $46 \%$ vs. $21 \%$ ), a defined primary outcome ( $56 \%$ vs. $39 \%$ ), and involved multiple centres (46\% vs. 16\%). Financial support remained significantly associated with these factors in multivariable models (Table 4). In addition, financial support increased the frequency of reporting clinical outcomes in multivariable models. There was little change in design quality over time, other than a decrease in the proportion of studies with prospective data collection $(77 \%$ in $1977-2004,67 \%$ in 2005-2010, 42\% in 2011-2013, 40\% in 2014-2015, 39\% in 2016-2017) and an increase in studies reporting a clinical outcome (39\% in 1977-2004, 44\% in 2005-2010, 44\% in 2011-2013, 53\% in 2014-2015, 59\% in 2016-2017)) (Figure S1D). These outcomes were significantly associated with calendar time in multivariable models (Table 4), and sample size calculations were independently reported more in later studies. The decrease in studies with prospective data collection is most prominent in studies in the hospital setting (Table S2). There were no large differences between studies performed in children versus adults (Figure S1C). Geographical region was independently associated with randomized designs, using an external control, prospective data collection, performing sample size calculations, reporting a primary, clinical or microbiological outcome, and being multicentre (Table 4). 
Table 4 Results of stepwise backward selection of multivariable model containing all the factors with the different design quality indicators as outcome. The colours indicate either a strong negative association $(O R<1.0)$ in red, or a strong positive association $(O R>1.0)$ in green. Numbers indicate odds ratios with $95 \%$ confidence intervals.

\begin{tabular}{|c|c|c|c|c|c|c|c|c|c|c|c|}
\hline \multirow{2}{*}{\multicolumn{2}{|c|}{ Factors }} & \multicolumn{10}{|c|}{ Design quality indicators } \\
\hline & & $\begin{array}{c}\text { Model } 1 \\
\text { Randomised } \\
\text { design }\end{array}$ & \begin{tabular}{|c|} 
Model 2 \\
External control
\end{tabular} & $\begin{array}{c}\text { Model } 3 \\
\text { Sample size } \\
\text { calculation }\end{array}$ & \begin{tabular}{|c|} 
Model 4 \\
Prospective data
\end{tabular} & $\begin{array}{l}\text { Model } 5 \\
\text { Confounding } \\
\text { correction }\end{array}$ & $\begin{array}{l}\text { Model } 6 \\
\text { Primary } \\
\text { outcome }\end{array}$ & \begin{tabular}{c|} 
Model 7 \\
Clinical outcome
\end{tabular} & $\begin{array}{c}\text { Model } 8 \\
\begin{array}{l}\text { Microbiological } \\
\text { outcome }\end{array}\end{array}$ & $\begin{array}{l}\text { Model } 9 \\
\text { Multicentre }\end{array}$ & $\begin{array}{c}\text { Model } 10 \\
\text { Sustainability } \\
\text { assessed }\end{array}$ \\
\hline Clinical setting & Community (n=205) & $5.9(3.8-9.2)$ & $5.6(3.6-8.5)$ & $2.9(1.9-4.5)$ & $1.7(1.2-2.6)$ & $3.0(2.1-4.3)$ & $1.4(1.0-2.0)$ & $0.3(0.2-0.5)$ & $0.2(0.1-0.4)$ & $10.5(7.1-15.7)$ & \\
\hline Financial support & Yes $(n=385)$ & $4.7(2.9-7.5)$ & $4.1(2.6-6.3)$ & $3.1(2.1-4.7)$ & $2.2(1.6-3.0)$ & $2.5(1.8-3.4)$ & $1.8(1.4-2.5)$ & $1.9(1.4-2.6)$ & & $3.0(2.0-4.3)$ & \\
\hline \multirow[t]{2}{*}{ Age group studied** } & Children (n=101) & $1.1(0.6-2.2)$ & $0.8(0.4-1.6)$ & $0.8(0.5-1.4)$ & $1.1(0.7-1.9)$ & & $1.0(0.6-1.6)$ & $0.6(0.4-1.0)$ & & & $1.4(0.9-2.2)$ \\
\hline & Both $(n=375)$ & $0.6(0.4-1.0)$ & $0.6(0.4-0.9)$ & $0.4(0.2-0.6)$ & $0.5(0.4-0.7)$ & & $0.5(0.4-0.7)$ & $0.4(0.3-0.5)$ & & & $1.6(1.2-2.2)$ \\
\hline \multicolumn{2}{|c|}{ Year study conducted ${ }^{* \star \star} 2005-2010(n=160)$} & $1.0(0.5-1.9)$ & $1.1(0.6-1.9)$ & $2.5(1.3-4.8)$ & $0.4(0.2-0.6)$ & $0.6(0.4-1.0)$ & & $0.9(0.6-1.5)$ & $2.1(1.2-3.7)$ & & $1.1(0.7-1.8)$ \\
\hline & $2011-2013(n=156)$ & $0.5(0.3-1.1)$ & $0.5(0.2-0.9)$ & $1.8(0.9-3.5)$ & $0.2(0.1-0.3)$ & $0.7(0.4-1.2)$ & & $1.0(0.6-1.6)$ & $1.7(1.0-3.0)$ & & $1.6(1.0-2.5)$ \\
\hline & $2014-2015(n=173)$ & $0.4(0.2-0.8)$ & $0.5(0.2-0.9)$ & $2.5(1.3-4.9)$ & $0.1(0.1-0.2)$ & $1.1(0.7-1.8)$ & & $1.3(0.8-2.1)$ & $1.2(0.7-2.1)$ & & $1.8(1.1-2.8)$ \\
\hline & 2016-2017 (n=176) & $0.8(0.4-1.5)$ & $0.9(0.5-1.7)$ & $2.5(1.3-4.9)$ & $0.1(0.1-0.2)$ & $1.2(0.7-1.9)$ & & $1.7(1.0-2.7)$ & $1.4(0.8-2.4)$ & & $1.2(0.8-1.8)$ \\
\hline \multirow[t]{5}{*}{ Geographical region } & Europe $(n=260)$ & $2.5(1.5-4.2)$ & $2.2(1.4-3.5)$ & $1.6(1.1-2.5)$ & $2.8(1.9-4.1)$ & & $1.1(0.8-1.5)$ & $1.1(0.8-1.6)$ & $0.9(0.6-1.4)$ & $2.0(1.3-3.1)$ & \\
\hline & Asia $(n=128)$ & $2.6(1.3-5.0)$ & $2.4(1.3-4.4)$ & $1.1(0.6-2.0)$ & $1.7(1.1-2.7)$ & & $0.4(0.3-0.7)$ & $1.8(1.1-2.8)$ & $1.2(0.8-2.0)$ & $1.0(0.6-1.8)$ & \\
\hline & Oceania $(n=36)$ & $1.6(0.6-4.2)$ & $1.5(0.6-3.7)$ & $0.5(0.1-1.4)$ & $1.8(0.8-3.9)$ & & $0.5(0.2-1.0)$ & $0.5(0.2-1.0)$ & $0.2(0.1-0.8)$ & $0.9(0.4-2.2)$ & \\
\hline & Africa (n=29) & $4.0(1.1-14.5)$ & $3.3(0.9-11.8)$ & $1.8(0.5-5.8)$ & $2.0(0.6-6.2)$ & & $0.9(0.3-2.5)$ & $1.8(0.6-5.3)$ & $0.2(0.1-1.7)$ & $7.2(2.0-25.1)$ & \\
\hline & South America $(n=17)$ & $1.8(0.4-7.4)$ & $2.3(0.6-8.4)$ & $2.0(0.6-6.5)$ & $2.2(0.8-6.4)$ & & $1.1(0.4-2.9)$ & $1.1(0.4-3.0)$ & $1.3(0.4-3.8)$ & & \\
\hline
\end{tabular}

*Reference category: studies performed in the hospital setting ( $n=620)$, "Reference category: studies performed in adults ( $n=349)$, ** Reference category: studies performed before 2005 ( $n=160)$, **** Reference category: studies performed in North America $(n=368)$

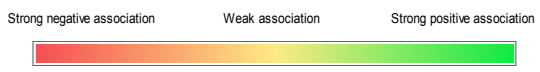

\section{DISCUSSION}

In previous systematic reviews of antimicrobial stewardship evaluations, many studies have been excluded because of not fulfilling minimal methodological quality criteria. We have undertaken the first comprehensive systematic review focusing on describing quality, rather than excluding studies based on quality, to facilitate formulating recommendations for improvement. In addition, we evaluated quality features required for validity and translation into practice instead of focusing solely on methodological quality. Our systematic review revealed that the design quality of antimicrobial stewardship evaluations is low, with only a minority of studies reporting clinical and microbiological outcome data. Design quality is considerably better in studies performed in the community setting. We find published evaluations provide a striking lack of evidence for the clinical and microbiological impacts of antimicrobial stewardship interventions. The majority of studies focus exclusively on process measures. While it is clearly essential to establish whether an intervention is effective in changing antibiotic use, reporting clinical outcomes is crucial to assess the safety of antimicrobial stewardship interventions ${ }^{[16,17]}$. The clinical outcomes reported often utilize routinely collected data, which may explain the differences between the community and hospital setting. In particular, in the hospital setting, commonly used clinical outcomes are mortality and length of hospital stay ${ }^{[18,19]}$. 
As indicated by the observed time trends, these outcomes are being used with increasing frequency, probably because extraction of relevant data from electronic health records is becoming more feasible. Such routinely available data are not the most sensitive and patient-relevant outcomes. In the hospital setting, markers of early treatment response such as clinical stability may be preferable. In the community setting, repeat consultations, relapse of infection, and hospital admissions may be more relevant; yet data on these outcomes are not routinely collected ${ }^{[20-22]}$.

Very few stewardship studies report microbiological outcomes. This is surprising given that reducing antimicrobial resistance is the ultimate goal of antimicrobial stewardship. However, this is consistent with a meta-analysis on the effect of stewardship interventions on infection and colonization with antibiotic-resistant bacteria and Clostridium difficile infections that showed the literature on this topic is sparse and dominated by low-quality research ${ }^{[23]}$. Some authorities have called for stewardship evaluations to routinely include consideration of the impact of stewardship on resistance ${ }^{[24]}$ but studies generally lack power to determine this. Relationships between antimicrobial exposure and resistance may be more efficiently established through specific mechanistic studies rather than within stewardship evaluations.

Our analysis demonstrates that factors that would be expected to affect study design do, while others do not. The contrast between the community and hospital setting is striking in terms of the greater use of multicentre, randomized controlled designs. One explanation could be that clusters required for cluster randomization are more readily available in the community setting. In contrast, clinical outcomes and microbiological data are less readily available in the community setting. Retrospective study designs are therefore less feasible in the community setting.

Financial support was associated with better design quality. In addition to the costs inherent to conducting multicentre, prospective studies with longer follow-up, the process of securing funding may drive careful consideration of study validity. Less than half of the stewardship studies reviewed reported external funding. However, our finding of an association between external funding and improved design quality underscores the necessity of external funding to support appropriate implementation and robust evaluation of antimicrobial stewardship programmes ${ }^{[25]}$.

Our results show that there is no improvement of design quality over time, which is in contrast to previous reports ${ }^{[5,23]}$. This may be explained by our evaluation and inclusion of all studies without a pre-selection on study design, while previous reviews only included 
adequate studies with interpretable data ${ }^{[5]}$.

In keeping with previous reports, we found that the majority of antimicrobial stewardship studies used non-randomized research designs, with before-after studies being the most prevalent. This quasi-experimental research design is commonly used for quality improvement projects. However, such studies are at risk from multiple forms of bias ${ }^{116,24,}$ ${ }^{26]}$ and the Effective Practice and Organisation of Care (EPOC) criteria strongly discourages the inclusion of before-after studies without an ITS analysis in systematic reviews ${ }^{[13]}$. Incorporation of properly conducted ITS analysis into before-after studies has the potential to robustly control for time-dependent bias ${ }^{[27]}$ but only $20 \%(104 / 515)$ of before-after studies we identified used ITS analysis. Moreover, it has been shown that contemporary ITS analyses are often performed with an insufficient number of data points [11].

This systematic review has several strengths. First, our comprehensive search strategy gives a unique overview of the quality of studies evaluating antimicrobials stewardship interventions. Second, we used the PRISMA reporting guide for systematic reviews ${ }^{[15]}$. Third, the quality indicators and candidate factors were selected in a consensus procedure as part of a Joint Programming Initiative on Antimicrobial Resistance (JPIAMR)funded consensus group, which was selected to both include experts on the field of antimicrobial stewardship and trial methodology. Finally, this is the first comprehensive systematic review to determine the extent to which published antimicrobial stewardship evaluations include quality features required for validity and translation into practice.

The limitations of our review were, firstly, we only searched PubMed and excluded nonEnglish studies, which makes it possible that antimicrobial stewardship studies indexed elsewhere and non-English studies were missed. However, if we compare the studies identified by our searching strategy with the largest community and hospital stewardship systematic reviews, only six studies were missed because of being indexed elsewhere and 11 because of being non-English. Therefore, this is likely to have had a minimal impact on the total results ${ }^{[5,12]}$. Secondly, the screening, inclusion, and data extraction were performed by only one investigator, which could have resulted in studies being missed, wrongly included, or misclassification of the extracted data. To estimate the number of studies that might be missed, a proportion of the studies was screened and data were extracted by a second author. In this second round, we showed that the percentage of agreement was high, with a moderate interobserver agreement. Assuming that every disagreement in included studies would have inadvertently excluded a study (1\% of 700 studies reviewed twice), we may have missed a maximum of 104 inclusions in the other studies not screened twice. In a systematic review with meta-analysis, the consequence 
of missing or wrongly including a single study could have a large impact on the pooled effect estimate. However, as we did not focus on the outcome of individual studies but rather on the design quality of many studies, given the large number of studies included, it is unlikely that the missed studies would have changed the conclusions. And lastly, the quality indicator definition of a primary outcome was only based on what was described in the manuscript. It is possible that a primary outcome was defined retrospectively based on the observed data in a proportion of the studies.

Concerns about the methodological quality of antimicrobial stewardship studies have been raised before, with several publications making recommendations to improve their scientific methods $[16,24,26]$. However, have found no improvement in methodological quality over time except for more frequent inclusion of sample size calculation and clinical outcomes. Therefore, there is still a need for clear recommendations to improve antimicrobial stewardship design quality. Recommendations for improvement should especially consider (1) emphasizing the importance of choosing appropriate clinical and microbiological outcomes, (2) focusing on robust methods to evaluate stewardship interventions in the hospital setting. Implementing these recommendations in future antimicrobial stewardship studies will help in the optimal use of resources to determine which stewardship interventions are most effective to change clinical practice. Building on the work from the systematic review we established a working group of expert investigators in this field. This systematic review identifies the limitations in design features that are most important for validity and translation into clinical practice. The results will be used to formulate recommendations in a white paper that will support investigators with key design decisions, support funders assessing proposals for stewardship studies and enhance the quality and impact of research in this crucial area.

\section{List of abbreviations}

AMR, antimicrobial resistance; EPOC, Effective Practice and Organisation of Care; ITS, Interrupted time-series; JPIAMR, Joint Programming Initiative on Antimicrobial Resistance.

\section{Acknowledgements}

Other members of the Consensus on Antimicrobial Stewardship Evaluations (CASE) study group: S.Harbarth, B. Huttner, P. Little, J. Rodriguez-Baño, A. Savoldi, M. van Smeden, E. Tacconelli, J.F. Timsit, M. Wolkewitz.

\section{Conflicts of interest}

The authors declare no conflict of interest. The presented work was supported by a grant (JPIAMRWG-010) from the Joint Programming Initiative on Antimicrobial Resistance. 


\section{REFERENCES}

1. O'Neill J. Review on Antimicrobial Resistance. Tackling Drug-Resistant Infect Glob Final Rep Recomm 2016. https://amr-review.org/sites/default/files/160518_Final paper_with cover.pdf.

2. Goossens H, Ferech M, Vander Stichele R, Elseviers M, ESAC Project Group H, Sprenger M, et al. Outpatient antibiotic use in Europe and association with resistance: a cross-national database study. Lancet (London, England) 1998;365:579-87. doi:10.1016/S0140-6736(05)17907-0.

3. Costelloe C, Metcalfe C, Lovering A, Mant D, Hay AD. Effect of antibiotic prescribing in primary care on antimicrobial resistance in individual patients: systematic review and meta-analysis. Bmj 2010;340:c2096. doi:10.1136/bmj.c2096.

4. Dyar OJ, Huttner B, Schouten J, Pulcini C, ESGAP (ESCMID Study Group for Antimicrobial stewardshiP). What is antimicrobial stewardship? Clin Microbiol Infect 2017;0. doi:10.1016/j.cmi.2017.08.026.

5. Davey P, Marwick CA, Scott CL, Charani E, Mcneil K, Brown E, et al. Interventions to improve antibiotic prescribing practices for hospital inpatients. Cochrane Database Syst Rev 2017;2017. doi:10.1002/14651858. CD003543.pub4

6. Molina J, Cisneros JM. A chance to change the paradigm of outcome assessment of antimicrobial stewardship programs. Clin Infect Dis 2015;61:807-8. doi:10.1093/cid/civ496.

7. Wagner B, Filice GA, Drekonja D, Greer N, MacDonald R, Rutks I, et al. Antimicrobial stewardship programs in inpatient hospital settings: a systematic review. Infect Control Hosp Epidemiol 2014;35:1209-28. doi:10.1086/678057

8. Schuts EC, Hulscher MEJL, Mouton JW, Verduin CM, Stuart JWTC, Overdiek HWPM, et al. Current evidence on hospital antimicrobial stewardship objectives: A systematic review and meta-analysis. Lancet Infect Dis 2016;16:847-56. doi:10.1016/S1473-3099(16)00065-7.

9. Ivers N, Jamtvedt G, Flottorp S, Young JM, Odgaard-Jensen J, French SD, et al. Audit and feedback: effects on professional practice and healthcare outcomes. Cochrane Database Syst Rev 2012;6:Art. No.: CD000259. DOI: 10.1002/14651858.CD000259. doi:10.1002/14651858.CD000259.pub3.

10. Charani E, Edwards R, Sevdalis N, Alexandrou B, Sibley E, Mullett D, et al. Behavior change strategies to influence antimicrobial prescribing in acute care: A systematic review. Clin Infect Dis 2011;53:651-62. doi:10.1093/cid/cir445.

11. Ramsay C, Brown E, Hartman G, Davey P. Room for improvement: A systematic review of the quality of evaluations of interventions to improve hospital antibiotic prescribing. J Antimicrob Chemother 2003;52:76471. doi:10.1093/jac/dkg460

12. Drekonja DM, Filice GA, Greer N, Olson A, MacDonald R, Rutks I, et al. Antimicrobial stewardship in outpatient settings: A systematic review. Infect Control Hosp Epidemiol 2015;36:142-52. doi:10.1017/ice.2014.41.

13. Effective Practice and Organisation of Care. No Title. EPOC Resour Rev Authors 2015:http://epoc.cochrane. org/resources/epoc-resources-.

14. Pulcini C, Huttner A. CMI policy on antimicrobial stewardship research. Clin Microbiol Infect 2017. doi:10.1016/j. cmi.2017.11.006. 
15. Moher D, Liberati A, Tetzlaff J ADPG. Preferred reporting items for systematic reviews and meta-analyses: the PRISMA statement. Bmj 2009.

16. McGowan JE. Antimicrobial Stewardship-the State of the Art in 2011 Focus on Outcome and Methods. Infect Control Hosp Epidemiol 2012;33:331-7. doi:10.1086/664755.

17. Gillespie D. Use of co-primary outcomes for trials of antimicrobial stewardship interventions. n.d

18. Horikoshi Y, Higuchi H, Suwa J, Isogai M, Shoji T, Ito K. Impact of computerized pre-authorization of broad spectrum antibiotics in Pseudomonas aeruginosa at a children's hospital in Japan. J Infect Chemother 2016;22:532-5. doi:10.1016/j.jiac.2016.05.001

19. Hohn A, Heising B, Hertel S, Baumgarten G, Hochreiter M, Schroeder S. Antibiotic consumption after implementation of a procalcitonin-guided antimicrobial stewardship programme in surgical patients admitted to an intensive care unit: a retrospective before-and-after analysis. Infection 2015;43:405-12. doi:10.1007/ s15010-014-0718-x.

20. Vellinga A, Galvin S, Duane S, Callan A, Bennett K, Cormican M, et al. Intervention to improve the quality of antimicrobial prescribing for urinary tract infection: a cluster randomized trial. CMAJ 2016;188:108-15. doi:10.1503/cmaj.150601.

21. Butler CC, Simpson SA, Dunstan F, Rollnick S, Cohen D, Gillespie D, et al. Effectiveness of multifaceted educational programme to reduce antibiotic dispensing in primary care: Practice based randomised controlled trial. BMJ 2012;344. doi:10.1136/bmj.d8173.

22. Persell SD, Doctor JN, Friedberg MW, Meeker D, Friesema E, Cooper A, et al. Behavioral interventions to reduce inappropriate antibiotic prescribing: A randomized pilot trial. BMC Infect Dis 2016;16. doi:10.1186/ s12879-016-1715-8.

23. Baur D, Gladstone BP, Burkert F, Carrara E, Foschi F, Döbele S, et al. Effect of antibiotic stewardship on the incidence of infection and colonisation with antibiotic-resistant bacteria and Clostridium difficile infection: a systematic review and meta-analysis. Lancet Infect Dis 2017;17:990-1001. doi:10.1016/S1473-3099(17)30325-0.

24. de Kraker MEA, Abbas M, Huttner B, Harbarth S. Good epidemiological practice: a narrative review of appropriate scientific methods to evaluate the impact of antimicrobial stewardship interventions. Clin Microbiol Infect 2017;23:819-25. doi:10.1016/j.cmi.2017.05.019

25. Pulcini C, Morel CM, Tacconelli E, Beovic B, de With K, Goossens H, et al. Human resources estimates and funding for antibiotic stewardship teams are urgently needed. Clin Microbiol Infect 2017;23:785-7. doi:10.1016/j.cmi.2017.07.013

26. McGregor JC, Furuno JP. Optimizing research methods used for the evaluation of antimicrobial stewardship programs. Clin Infect Dis 2014;59:S185-92. doi:10.1093/cid/ciu540.

27. Cantey JB, Wozniak PS, Pruszynski JE, Sánchez PJ. Reducing unnecessary antibiotic use in the neonatal intensive care unit (SCOUT): a prospective interrupted time-series study. Lancet Infect Dis 2016;16:1178-84. doi:10.1016/S1473-3099(16)30205-5. 


\section{SUPPLEMENTARY MATERIAL}

Appendix 1 Search strategy in PubMed

("anti-infective agents"[Mesh] OR anti-infective*[tiab] OR antiinfective*[tiab] OR microbicides*[tiab] OR antimicrobial*[tiab] OR anti-microbial*[tiab] OR anti-bacterial*[tiab] OR antibacterial*[tiab] OR antibiotic*[tiab] OR antimycobacterial*[tiab] OR "antifungal agents"[Mesh] OR antifungal*[tiab] OR anti-fungal*[tiab]) AND (stewardship[tiab] OR restrict*[tiab] OR educat*[tiab] OR reminder*[tiab] OR (audit[tiab] OR audit'[tiab] OR audit's[tiab] OR audit1[tiab] OR audit2[tiab] OR audit4[tiab] OR auditary[tiab] OR auditbase[tiab] OR audited[tiab] OR audited' [tiab] OR auditee[tiab] OR auditees[tiab] OR auditer[tiab] OR auditing[tiab] OR auditing'[tiab] OR auditing's[tiab] OR auditings[tiab] OR auditor[tiab] OR auditor' [tiab] OR auditor's[tiab] OR auditors[tiab] OR auditors'[tiab] OR audits[tiab] OR audits'[tiab] OR auditsystem[tiab]) OR feedback*[tiab] OR "order forms"[tiab] OR approval*[tiab] OR detailing*[tiab] OR ((short[tiab] OR shorten[tiab] OR shorted[tiab] OR shortened[tiab] OR shortening[tiab] OR shortens[tiab] OR shorter[tiab]) AND duration[tiab]) OR ((stop[tiab] OR stops[tiab] OR stopped[tiab] OR stopping[tiab]) AND duration[tiab])) AND (intervention*[tiab] OR study[tiab] OR before-after*[tiab] OR (trial[tiab] OR trials[tiab]) OR "interrupted time series"[tiab]) NOT ("HIV Infections"[Mesh] OR HIV[Mesh] OR HIV[ti] OR human immunodeficiency virus[ti]) 
Appendix 2 Standard data extraction template

Methods:

- $\quad$ Title/abstract screening

- Titles will be screened by one author

- Papers selected by at least one author are selected for full text screening

- A random selection of papers will be screened by a second author and agreement will be assessed

- $\quad$ Full text screening

- Full text will be screened by one author

- Any uncertainties on inclusion or exclusion will be resolved based on a discussion on with all the authors

- Inclusion criteria

- Study domain: papers evaluating stewardship interventions

- Determinant: antibiotic stewardship intervention of any type

- Study design: no restriction

- Language: English

- Database: PubMed

- Exclusion criteria

- Case reports

- Narrative or systematic reviews*

- Discussion papers*

* Narrative or systematic reviews and discussion papers will be used for snowballing

Data extraction template:

\begin{tabular}{|c|c|}
\hline Variable & Specification \\
\hline Study ID & $\ldots$ \\
\hline Number in search file & $\ldots$ \\
\hline Manuscript title & $\ldots$ \\
\hline Publication year & $\ldots$ \\
\hline Publication journal & $\ldots$ \\
\hline Main author & $\ldots$ \\
\hline Age category & Adults/children/both \\
\hline Setting of study & Primary care/All hospital/ER/non-ICU ward/ICU/other \\
\hline Infectious disease(s) studied (multiple answers allowed) & $\begin{array}{l}\text { Upper respiratory tract infections/Lower respiratory tract infections/ } \\
\text { Abdominal infections/Sepsis/Prophylactic treatment/Bacteraemia/ } \\
\text { Urinary tract infections/None specified/Other, please specify }\end{array}$ \\
\hline Other infectious disease, specified & $\ldots$ \\
\hline Antibiotic class targeted (multiple answers allowed) & $\begin{array}{l}\text { Cephalosporins/Carbapenems/Penicillins/Fluoroquinolones/ } \\
\text { Aminoglycosides/Macrolides/None specified/Other, please specify }\end{array}$ \\
\hline Other antibiotic class, specified & $\ldots$ \\
\hline
\end{tabular}


Data extraction template continued.

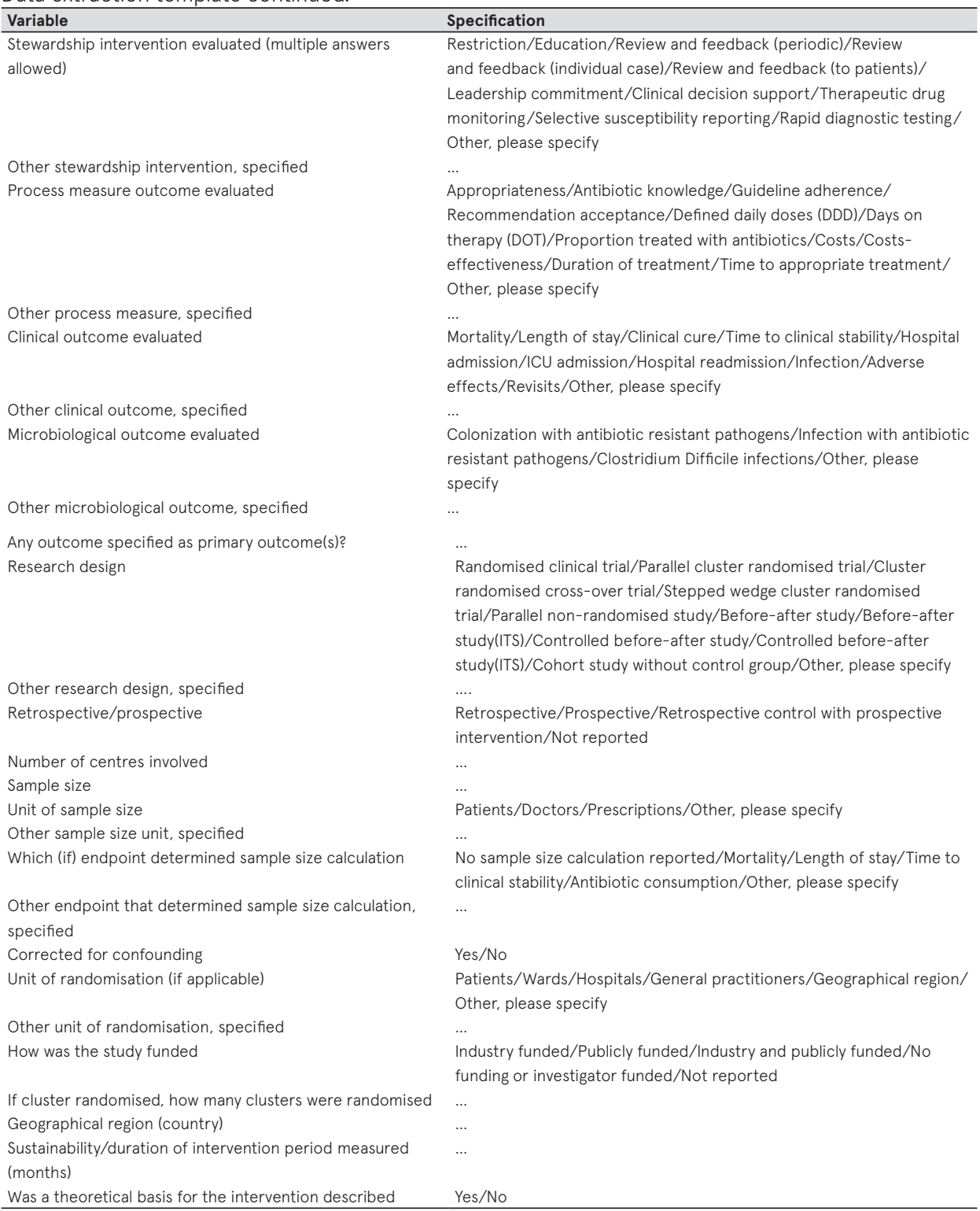


Table S1 Definition of the design quality determinants used and corresponding categorisation if applicable

\begin{tabular}{|c|c|c|}
\hline Design quality indicator & Definition & Categorisation (if applicable) \\
\hline Randomised research design & $\begin{array}{l}\text { Allocation of the antimicrobial stewardship } \\
\text { intervention or comparator was random. } \\
\text { Randomisation is defined as control by means of } \\
\text { random allocation at any level (individual or cluster). }\end{array}$ & $\begin{array}{l}\text { Randomised: } \\
\text { RCT, c-RCT, cx-RCT, sw-CRT } \\
\text { Non-randomised: } \\
\text { BA(with or without ITS analysis), cBA, } \\
\text { cohort studies, non-randomised trials }\end{array}$ \\
\hline External control group & $\begin{array}{l}\text { The outcome was also assessed in an external } \\
\text { control group without antimicrobial stewardship } \\
\text { intervention. External indicates that a } \\
\text { contemporaneous cluster or group was included } \\
\text { in which the intervention under study is not } \\
\text { implemented. }\end{array}$ & $\begin{array}{l}\text { Parallel control group: } \\
\text { CBA, RCT, non-randomised trials, c-RCT, } \\
\text { CX-RCT, sw-CRT } \\
\text { No parallel control group: } \\
\text { BA, cohort studies }\end{array}$ \\
\hline Number of centres & $\begin{array}{l}\text { Amount of centres involved in the study, either as } \\
\text { control or intervention. }\end{array}$ & $\begin{array}{l}\text { Single centre: } 0-1 \text { centre } \\
\text { Multicentre: }>1 \text { centre }\end{array}$ \\
\hline Sample size calculation reported & $\begin{array}{l}\text { A sample size calculation was performed to ensure } \\
\text { sufficient power for the primary outcome. }\end{array}$ & - \\
\hline Prospective data collection & $\begin{array}{l}\text { The data was prospectively collected. If not } \\
\text { reported we assumed the data collection to be } \\
\text { retrospective. }\end{array}$ & - \\
\hline Confounding correction & $\begin{array}{l}\text { The intervention effect was corrected for } \\
\text { confounding bias, either by randomisation, } \\
\text { matching, stratification or correction. }\end{array}$ & - \\
\hline
\end{tabular}

RCT: randomised controlled trial, C-RCT: parallel cluster randomised controlled trial, cx-RCT: cluster cross-over randomised controlled trial, sw-CRT: stepped wedge cluster randomised trial, BA: before-after study, cBA: controlled before-after study, ITS: interrupted time-series, CDI: Clostridium difficile infection 
Appendix 3 PRISMA criteria for the reporting of systematic reviews

\begin{tabular}{|c|c|c|c|}
\hline Section/topic & \# & Checklist item & Reported on page \# \\
\hline \multicolumn{4}{|l|}{ TITLE } \\
\hline Title & 1 & Identify the report as a systematic review, meta-analysis, or both. & 1 \\
\hline \multicolumn{4}{|l|}{ ABSTRACT } \\
\hline Structured summary & 2 & $\begin{array}{l}\text { Provide a structured summary including, as applicable: background; } \\
\text { objectives; data sources; study eligibility criteria, participants, } \\
\text { and interventions; study appraisal and synthesis methods; results; } \\
\text { limitations; conclusions and implications of key findings; systematic } \\
\text { review registration number. }\end{array}$ & $2-3$ \\
\hline \multicolumn{4}{|l|}{ INTRODUCTION } \\
\hline Rationale & 3 & $\begin{array}{l}\text { Describe the rationale for the review in the context of what is already } \\
\text { known. }\end{array}$ & 4 \\
\hline Objectives & 4 & $\begin{array}{l}\text { Provide an explicit statement of questions being addressed with } \\
\text { reference to participants, interventions, comparisons, outcomes, and } \\
\text { study design (PICOS). }\end{array}$ & 4 \\
\hline
\end{tabular}

\section{METHODS}

Protocol and registration

Eligibility criteria

Information sources

Search

Study selection

Data collection process

Data items

Risk of bias in individual studies

Summary measures

Synthesis of results

Risk of bias across studies

Additional analyses

\section{RESULTS}

Study selection

Study characteristics

Risk of bias within studies
5 Indicate if a review protocol exists, if and where it can be accessed (e.g., Web address), and, if available, provide registration information including registration number.

6 Specify study characteristics (e.g., PICOS, length of follow-up) and report characteristics (e.g., years considered, language, publication status) used as criteria for eligibility, giving rationale.

7 Describe all information sources (e.g., databases with dates of coverage, contact with study authors to identify additional studies) in the search and date last searched.

8 Present full electronic search strategy for at least one database, including any limits used, such that it could be repeated.

9 State the process for selecting studies (i.e., screening, eligibility, included in systematic review, and, if applicable, included in the meta-analysis).

10 Describe method of data extraction from reports (e.g., piloted forms, 5 independently, in duplicate) and any processes for obtaining and confirming data from investigators.

11 List and define all variables for which data were sought (e.g., PICOS, funding sources) and any assumptions and simplifications made.

12 Describe methods used for assessing risk of bias of individual studies (including specification of whether this was done at the study or outcome level), and how this information is to be used in any data synthesis.

13 State the principal summary measures (e.g., risk ratio, difference in means).

14 Describe the methods of handling data and combining results of studies, if done, including measures of consistency (e.g., $\left.I^{2}\right)$ for each meta-analysis.

15 Specify any assessment of risk of bias that may affect the cumulative evidence (e.g., publication bias, selective reporting within studies).

16 Describe methods of additional analyses (e.g., sensitivity or subgroup analyses, meta-regression), if done, indicating which were prespecified.

17 Give numbers of studies screened, assessed for eligibility, and included in the review, with reasons for exclusions at each stage, ideally with a flow diagram. 18 For each study, present characteristics for which data were extracted N/A (e.g., study size, PICOS, follow-up period) and provide the citations.

19 Present data on risk of bias of each study and, if available, any $\mathrm{N} / \mathrm{A}$ Figure 1

6

N/A

6 /A outcome level assessment (see item 12).

Appendix 2

Appendix 1 
Appendix 3 continued.

\begin{tabular}{|c|c|c|c|}
\hline Section/topic & \# & Checklist item & Reported on page \# \\
\hline \multicolumn{4}{|l|}{ RESULTS } \\
\hline Results of individual studies & 20 & $\begin{array}{l}\text { For all outcomes considered (benefits or harms), present, for each } \\
\text { study: (a) simple summary data for each intervention group (b) effect } \\
\text { estimates and confidence intervals, ideally with a forest plot. }\end{array}$ & $7-9$ \\
\hline Synthesis of results & 21 & $\begin{array}{l}\text { Present results of each meta-analysis done, including confidence } \\
\text { intervals and measures of consistency. }\end{array}$ & N/A \\
\hline Additional analysis & 23 & $\begin{array}{l}\text { Give results of additional analyses, if done (e.g., sensitivity or subgroup } \\
\text { analyses, meta-regression [see Item 16]). }\end{array}$ & N/A \\
\hline \multicolumn{4}{|l|}{ DISCUSSION } \\
\hline Summary of evidence & 24 & $\begin{array}{l}\text { Summarize the main findings including the strength of evidence for } \\
\text { each main outcome; consider their relevance to key groups (e.g., } \\
\text { healthcare providers, users, and policy makers). }\end{array}$ & $10-12$ \\
\hline Conclusions & 26 & $\begin{array}{l}\text { Provide a general interpretation of the results in the context of other } \\
\text { evidence, and implications for future research. }\end{array}$ & 13 \\
\hline \multicolumn{4}{|l|}{ FUNDING } \\
\hline Funding & 27 & $\begin{array}{l}\text { Describe sources of funding for the systematic review and other } \\
\text { support (e.g., supply of data); role of funders for the systematic } \\
\text { review. }\end{array}$ & 14 \\
\hline
\end{tabular}

From: Moher D, Liberati A, Tetzlaff J, Altman DG, The PRISMA Group (2009). Preferred Reporting Items for Systematic Reviews and Meta-Analyses: The PRISMA Statement. PLoS Med 6(7): e1000097. doi:10.1371/journal.pmed1000097

For more information, visit: www.prisma-statement.org. 
Table S2 Design quality features changes over time of the included studies stratified by studies performed in the community and the hospital setting

\begin{tabular}{|c|c|c|c|c|c|}
\hline & 1977-2004 & $2005-2010$ & 2011-2013 & 2014-2015 & 2016-2017 \\
\hline \multicolumn{6}{|l|}{ Hospital } \\
\hline Randomised design & $10 \%$ & $17 \%$ & $6 \%$ & $6 \%$ & $8 \%$ \\
\hline External control & $12 \%$ & $20 \%$ & $6 \%$ & $8 \%$ & $11 \%$ \\
\hline Sample size calculation & $8 \%$ & $21 \%$ & $13 \%$ & $18 \%$ & $17 \%$ \\
\hline Multicentre & $9 \%$ & $14 \%$ & $18 \%$ & $20 \%$ & $18 \%$ \\
\hline Prospective data & $71 \%$ & $68 \%$ & $37 \%$ & $36 \%$ & $31 \%$ \\
\hline Confounding correction & $24 \%$ & $22 \%$ & $19 \%$ & $29 \%$ & $30 \%$ \\
\hline Primary outcome & $38 \%$ & $50 \%$ & $37 \%$ & $45 \%$ & $48 \%$ \\
\hline Clinical outcome & $43 \%$ & $51 \%$ & $50 \%$ & $58 \%$ & $66 \%$ \\
\hline Microbiological outcome & $21 \%$ & $38 \%$ & $32 \%$ & $23 \%$ & $27 \%$ \\
\hline Sustainability assessed & $53 \%$ & $50 \%$ & $55 \%$ & $65 \%$ & $54 \%$ \\
\hline \multicolumn{6}{|l|}{ Community } \\
\hline Randomised design & $43 \%$ & $47 \%$ & $41 \%$ & $41 \%$ & $61 \%$ \\
\hline External control & $45 \%$ & $51 \%$ & $41 \%$ & $45 \%$ & $65 \%$ \\
\hline Sample size calculation & $19 \%$ & $44 \%$ & $35 \%$ & $48 \%$ & $52 \%$ \\
\hline Multicentre & $68 \%$ & $75 \%$ & $78 \%$ & $69 \%$ & $71 \%$ \\
\hline Prospective data & $89 \%$ & $65 \%$ & $57 \%$ & $59 \%$ & $74 \%$ \\
\hline Confounding correction & $58 \%$ & $42 \%$ & $54 \%$ & $66 \%$ & $65 \%$ \\
\hline Primary outcome & $53 \%$ & $53 \%$ & $59 \%$ & $66 \%$ & $58 \%$ \\
\hline Clinical outcome & $32 \%$ & $29 \%$ & $27 \%$ & $31 \%$ & $29 \%$ \\
\hline Microbiological outcome & $6 \%$ & $5 \%$ & $8 \%$ & $17 \%$ & $10 \%$ \\
\hline Sustainability assessed & $43 \%$ & $56 \%$ & $78 \%$ & $55 \%$ & $52 \%$ \\
\hline
\end{tabular}


A

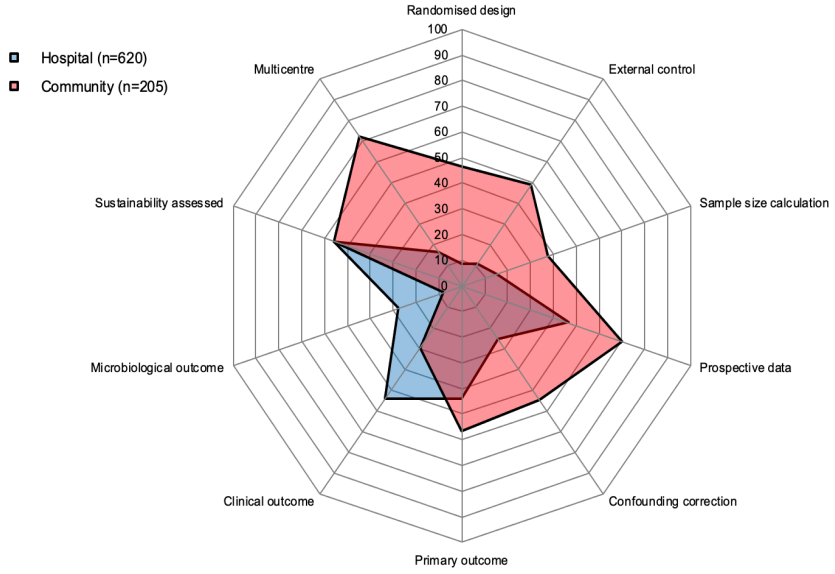

B

- No support $(\mathrm{n}=440)$

- Financial support $(n=385)$

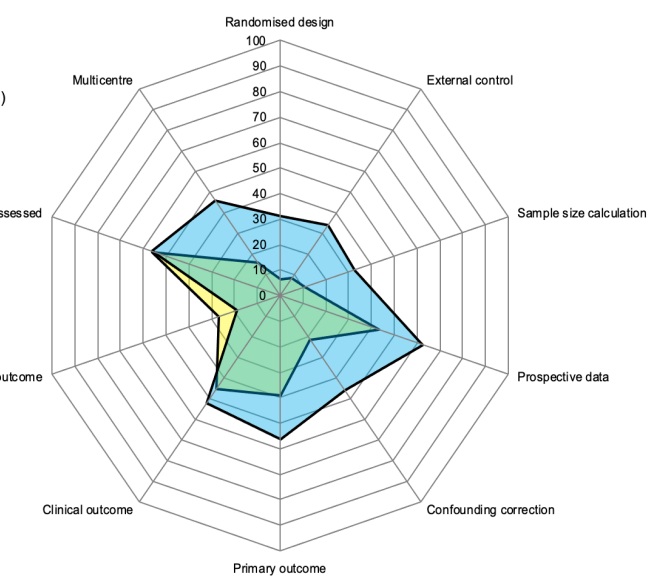

Figure S1 Design quality indicators stratified by factors: (A) community versus hospital setting, (B) financial support versus no financial support, (C) age setting: children, adults or both, (D) old versus new studies, (E) geographical region. 
C

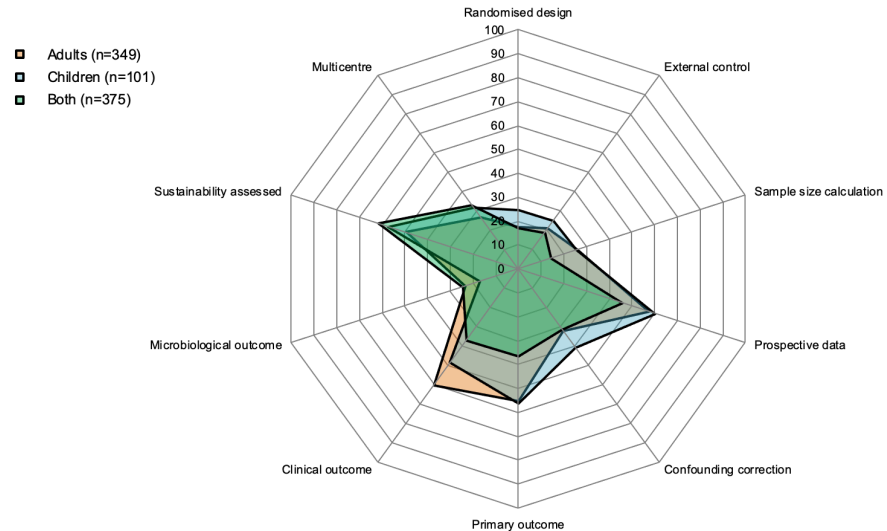

D

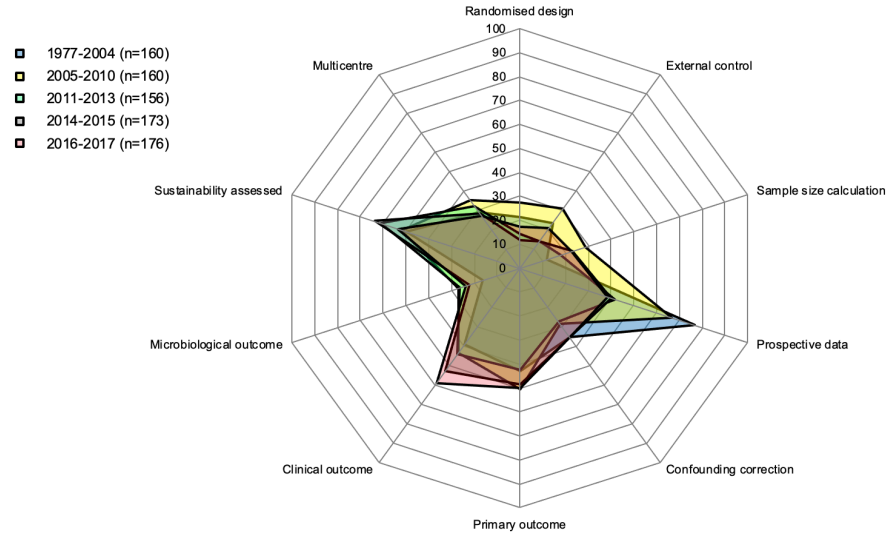

Figure S1 continued. 
E

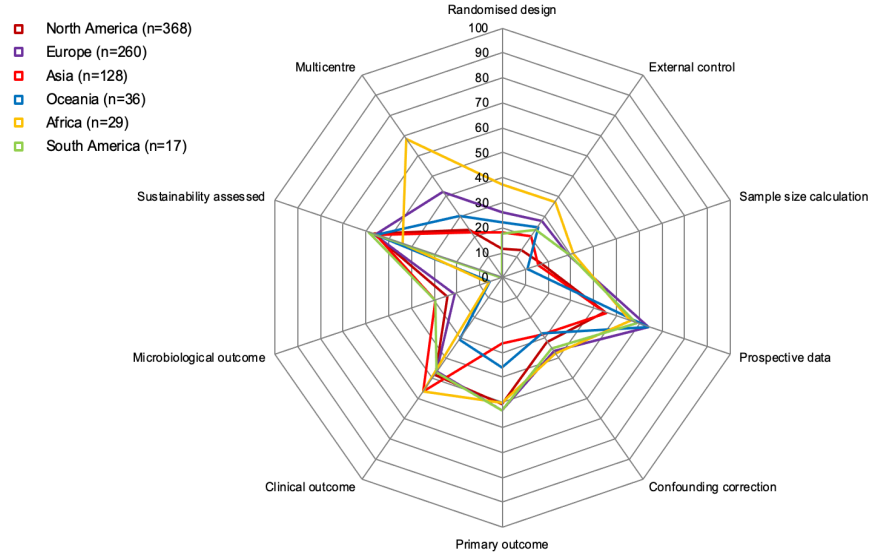

Figure $\mathbf{S} 1$ continued. 
Appendix 4 Literature list of included studies

\section{Online available at:}

https://www.sciencedirect.com/science/article/pii/S1198743X18307286?via\%3Dihub 
abo

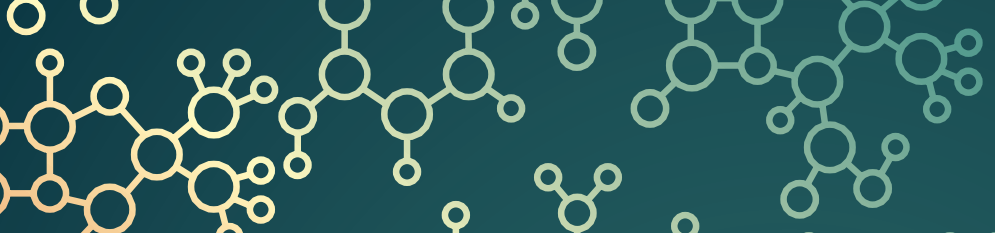
o

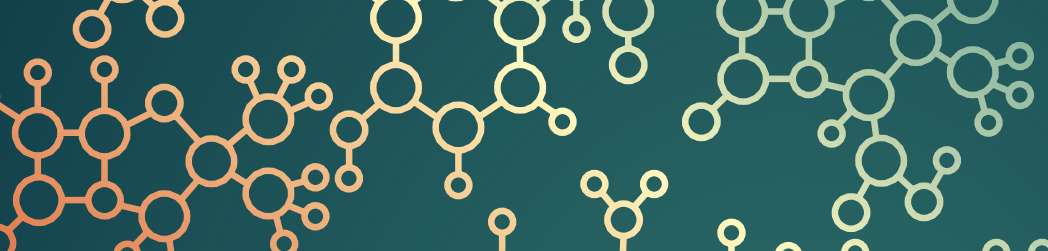

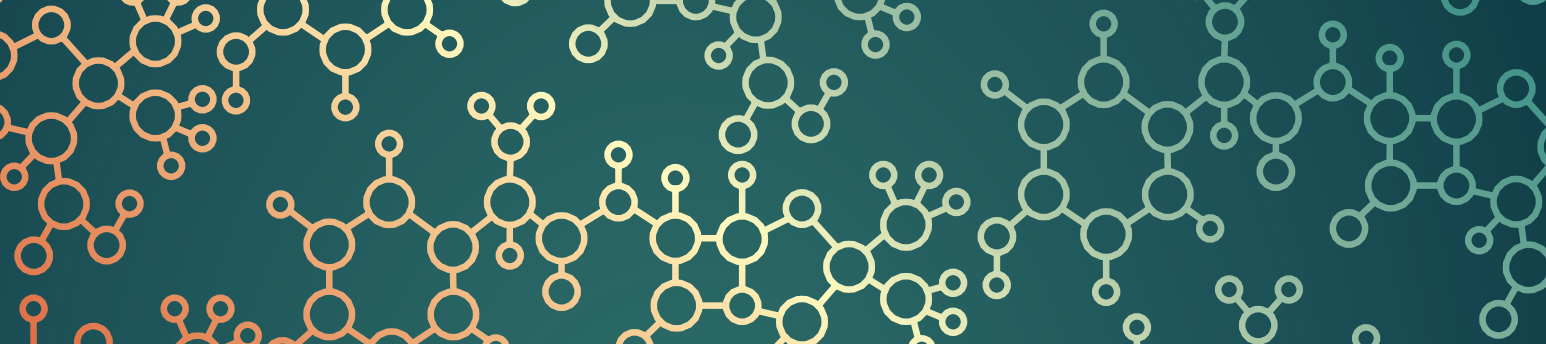

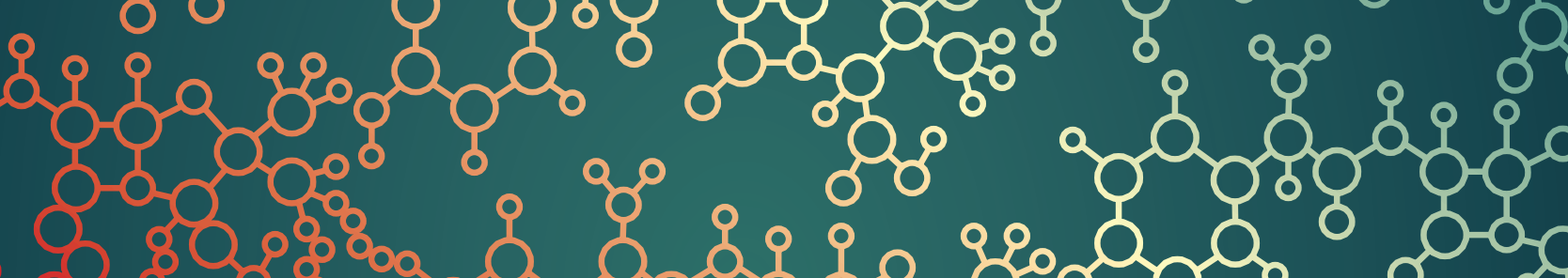
80\%

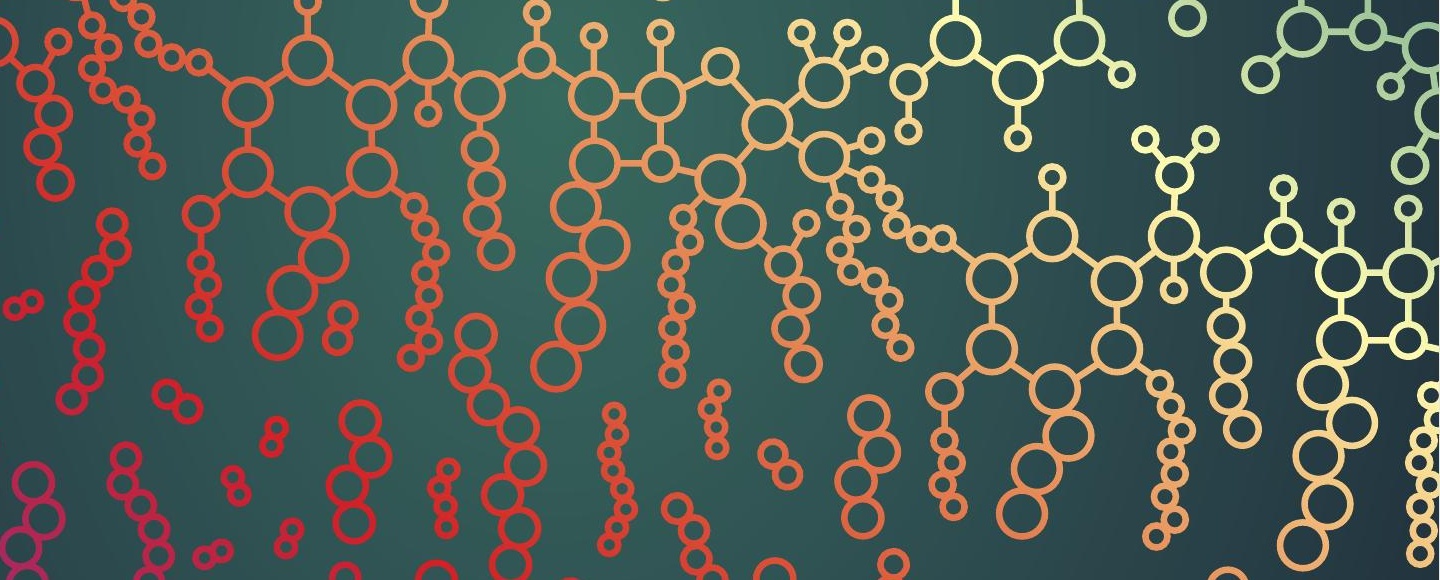
$88 \% 888$ $\circ 88$ \& 8

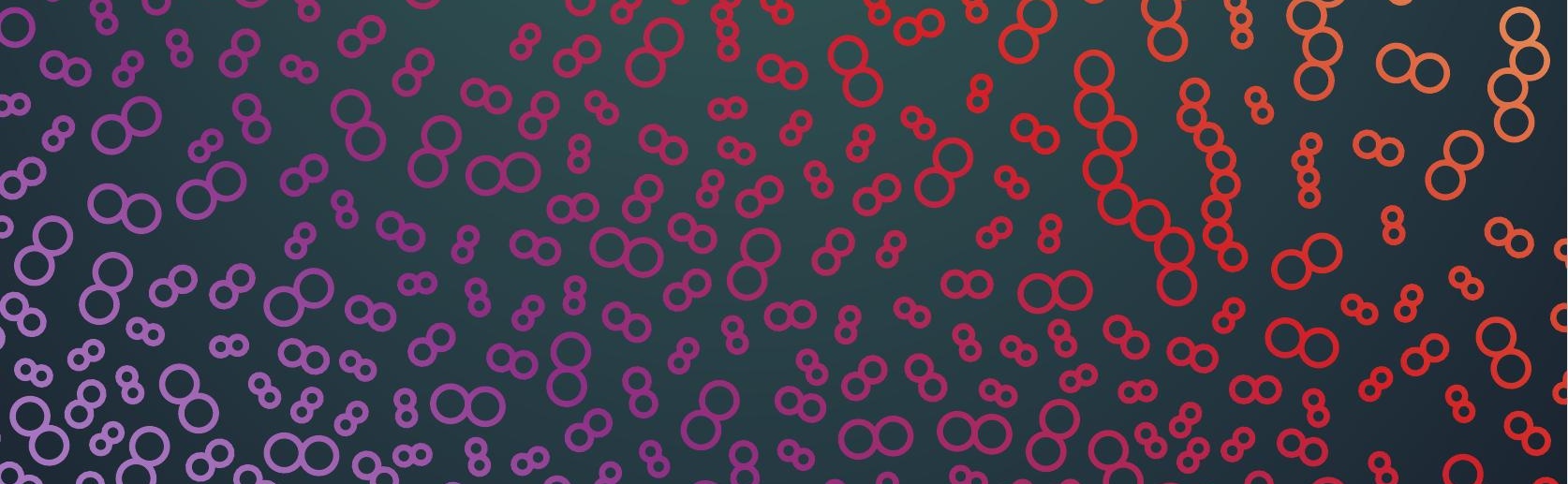




\section{Confounding by indication of the safety of de-escalation in community-acquired pneumonia: A simulation study embedded in a prospective cohort.}

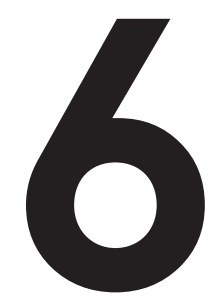

Inger van Heijl, Valentijn A. Schweitzer, C.H. Edwin Boel, Jan Jelrik Oosterheert, Susanne M. Huijts, Wendelien Dorigo-Zetsma, Paul D. van der Linden, Marc J.M. Bonten, Cornelis H. van Werkhoven 


\section{ABSTRACT}

\section{Background}

Observational studies have demonstrated that de-escalation of antimicrobial therapy is independently associated with lower mortality. This most probably results from confounding by indication. Reaching clinical stability is associated with the decision to de-escalate and with survival. However, studies rarely adjust for this confounder. We quantified the potential confounding effect of clinical stability on the estimated impact of de-escalation on mortality in patients with community-acquired pneumonia.

\section{Methods}

Data were used from the Community-Acquired Pneumonia immunization Trial in Adults (CAPITA). The primary outcome was 30-day mortality. We performed Cox proportionalhazards regression with de-escalation as time-dependent variable and adjusted for baseline characteristics using propensity scores. The potential impact of unmeasured confounding was quantified through simulating a variable representing clinical stability on day three, using data on prevalence and associations with mortality from the literature.

\section{Results}

Of 1,536 included patients, 257 (16.7\%) were de-escalated, 123 (8.0\%) were escalated and in $1156(75.3 \%)$ the antibiotic spectrum remained unchanged. Crude 30-day mortality was $3.5 \%$ (9/257) and $10.9 \%$ (107/986) in the de-escalation and continuation groups, respectively. The adjusted hazard ratio of de-escalation for 30-day mortality (compared to patients with unchanged coverage), without adjustment for clinical stability, was 0.39 (95\% Cl: $0.19-0.79)$. If $90 \%$ to $100 \%$ of de-escalated patients were clinically stable on day three, the fully adjusted hazard ratio would be $0.56(95 \% \mathrm{Cl}: 0.27-1.12)$ to $1.04(95 \% \mathrm{Cl}$ : 0.49-2.23), respectively. The simulated confounder was substantially stronger than any of the baseline confounders in our dataset.

\section{Conclusion}

Quantification of effects of de-escalation on patient outcomes without proper adjustment for clinical stability results in strong negative bias. This study suggests the effect of deescalation on mortality needs further well-designed prospective research to determine effect size more accurately. 


\section{INTRODUCTION}

The aim of antimicrobial stewardship is improving antibiotic use, without compromising clinical outcomes on the individual level ${ }^{[1]}$. De-escalation of empirical antimicrobial therapy is highly recommended in antimicrobial stewardship programs. In a recent systematic review de-escalation of empirical antimicrobial therapy was associated with a $56 \%$ (95\% $\mathrm{Cl} 34 \%-70 \%)$ relative risk reduction in mortality ${ }^{[2]}$. Although it seems a safe strategy, most studies evaluating de-escalation and reporting mortality were observational with a high risk of bias, high clinical heterogeneity and not sufficiently powered to demonstrate safety for mortality. To the best of our knowledge, there are two randomized trials evaluating de-escalation, and these trials did not show a survival benefit for de-escalation [3, 4]. A possible physiological mechanism for decreased mortality due to de-escalation could be a result of a more effective strategy by narrow-spectrum antibiotics or in case of continuation of unnecessary broad-spectrum antibiotics due to more (severe) sideeffects. However, it seems highly unlikely that this would lead to increased mortality in the population. Therefore, the association between de-escalation and improved survival in observational studies is most likely biased by unmeasured confounding by indication. Confounding by indication is present if the indication for the intervention (here: deescalation of empirical antimicrobial therapy) is also a prognostic factor for the outcome (mortality). De-escalation is usually only performed when clinical stability is reached in the first days after starting antimicrobial therapy and this also is a strong prognostic factor for patient outcome. However, hardly any of the observational studies adjusts for clinical stability during admission. In the aforementioned systematic review ${ }^{[2]}$ only one of nineteen observational studies corrected for this confounder ${ }^{[5]}$. Potentially they did not consider this to be an important confounder, or they lacked data on clinical stability during admission. Not taking this into account causes a negative bias (towards a protective effect). However, the magnitude of this bias has never been established. The aim of the current study was to quantify the potential effect of unmeasured confounding by indication due to clinical stability in the association between de-escalation and patient outcome in patients with community-acquired pneumonia.

\section{METHODS}

\section{Data collection}

Data were used from the Community-Acquired Pneumonia immunization Trial in Adults (CAPITA) ${ }^{[6]}$. This study was a parallel-group, randomized, placebo-controlled, double blind trial to assess the efficacy of a 13 -valent pneumococcal conjugate vaccine. The 
study included 84,496 immunocompetent community-dwelling adults, 65 years of age and above. Surveillance for suspected pneumonia was performed in 58 hospitals in the Netherlands, in the period September 2008 - August 2013. The study was approved by the Central Committee on Research Involving Human Subjects and by the Ministry of Health, Welfare and Sport in the Netherlands and all the participants provided written informed consent. For the current analysis, patients receiving antibiotics on the day of admission and with a working diagnosis of CAP admitted to a non-intensive care unit (ICU) were included. We think the effect of de-escalation on mortality in the ICU population is different than in non-ICU population and including these patients will result in a more heterogeneous population. Moreover, factors such as culture results and clinical stability may play a very different role in that population. Patients were excluded from the current analysis if they participated in a simultaneously running interventional trial evaluating different antibiotic regimens for CAP ${ }^{[7]}$, since this trial interfered with the choice of empirical antibiotic treatment, or if they died within 24 hours of admission because these are not eligible for de-escalation.

\section{Definitions}

To define de-escalation, antibiotics were ranked based on their spectrum of activity against CAP pathogens, from rank 1 ('narrow-spectrum') to rank 3 ('extended / restricted spectrum') antibiotics (Table 1). The ranking was performed by a team of experts: two clinical microbiologists (C.H.E.B., M.J.M.B.), one infectious diseases specialist (J.J.O.), two clinical pharmacists (I.v.H., P.D.v.d.L.) and one epidemiologist (C.H.v.W.). In the Dutch setting, penicillin and amoxicillin are in general classified as narrow-spectrum antibiotics. For mild CAP in primary care and moderate-severe CAP (non-ICU ward) these antibiotics are first choice treatment with tetracyclines as an alternative in case of allergies ${ }^{[8]}$. Sweden and Denmark have similar policies ${ }^{[9,10]}$. These antibiotics were classified as rank 1. Antibiotics with a 'restricted' label, advised by the national guide for antibiotic stewardship teams were classified as rank $3^{[11]}$. All other regimens were classified as rank 2. In patients with combination therapy, the highest rank of any individual antibiotic was counted, except for combination therapy of $\beta$-lactam therapy and a macrolide, which was considered as rank 3, as for respiratory pathogens this combination results in a much broader spectrum than any of the individual antibiotics. Therapy adjustment was defined as the first switch from empirical therapy to another antimicrobial class during hospitalization, independent of the reason for switching. De-escalation and escalation were defined as a change to a lower rank or a higher rank, respectively. Continued regimens or adjustments to an equivalent rank were defined as continuation. 
Table 1 Antibiotic ranking.

\begin{tabular}{lll}
\hline Rank 1 & Rank 2 & Rank 3 \\
(Narrow spectrum) & (Broad spectrum) & (Extended / restricted spectrum) \\
\hline Penicillin & $1^{\text {st }}$ generation cephalosporins & $3^{\text {d }}$ generation cephalosporins \\
Amoxicillin & $2^{\text {nd }}$ generation cephalosporins & $4^{\text {th }}$ generation cephalosporins \\
Tetracyclines & Co-amoxi-clav & Fluoroquinolones \\
& Co-trimoxazole & Aminoglycosides \\
& Clindamycin & Piperacillin/tazobactam \\
\hline
\end{tabular}

\section{Statistical analysis}

Descriptive statistics were used to describe clinical practice of de-escalation. Differences in patient characteristics between patients with a de-escalation versus no de-escalation were compared using Student's $t$ test or $x 2$ tests. Frequencies of de-escalation, escalation and continuation were described visually and numerically. We tested the proportional hazard assumptions for a follow-up period of 90 days, which revealed that the hazards were proportional up to 30 days and not thereafter (see Figure 1). Therefore we used 30-day mortality as the outcome. To determine the effect of de-escalation on clinical outcome we excluded patients starting in rank 1, since they are not able to deescalate. We performed Cox proportional hazards regression with de-escalation as timedependent variable and adjusted for baseline characteristics using propensity score analyses. Propensity scores were calculated from a logistic regression model to estimate a patients propensity for de-escalation and included the variables: age, gender, smoking status, history of diabetes mellitus, history of chronic pulmonary disease, antibiotic use two weeks before admission, rank on day 1, season of admission, weekday vs. weekend day (the latter defined as Saturday or Sunday), culture results and all variables from the Pneumonia Severity Index (PSI) score (nursing home resident, comorbidities (neoplastic disease, liver disease history, congestive heart failure history, cerebrovascular disease history, renal disease history), altered mental status, respiratory rate, systolic blood pressure, temperature, heart rate, $\mathrm{pH}$, blood urea nitrogen, sodium, glucose, hematocrit, partial pressure of oxygen and pleural effusion on x-ray). Propensity scores were then included as a continuous variable in the Cox proportional hazard regression model. Patients with escalation of therapy were censored at the time of escalation so that only the days before escalation contributed to the analysis. Other patients were censored at day 30 . 


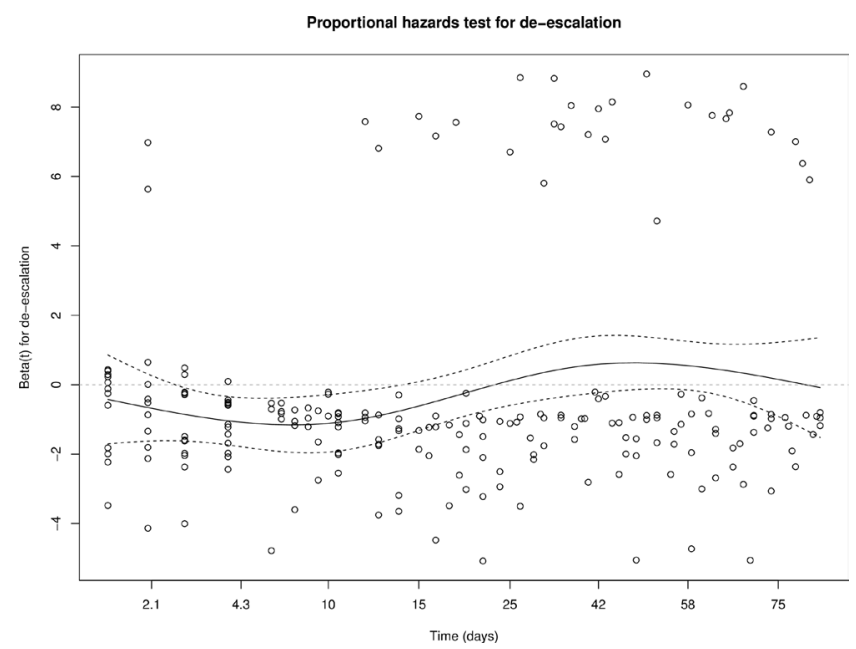

Figure 1 Proportional hazards test for de-escalation. The figure shows that the hazard ratio for deescalation is not constant over time (i.e. proportion hazards assumption violated): the association between de-escalation and mortality is present within approx. 20-days after admission but is around zero afterwards.

\section{Effect of confounding by indication}

To quantify the effect of unmeasured confounding by indication we simulated clinical stability during hospital admission as a new confounder. We defined clinical stability during admission as a binary variable evaluated at 72 hours, because clinical stability in patients with CAP is often reached within 48 hours and therapy is often evaluated after three days (with culture results also available) ${ }^{[8,12,13]}$. The strength of any given confounder is determined by the following three parameters: (1) the prevalence in the group with the determinant (de-escalation), (2) the prevalence in group without the determinant (continuation) and (3) the association with patient outcome (mortality). For the simulation of clinical stability at 72 hours we reviewed the literature for reasonable assumptions for the three parameters.

We assumed that $80 \%$ of CAP patients admitted to a non-ICU ward will be clinically stable at day three, based on three randomized controlled trials evaluating intravenous to oral switches in patients ${ }^{[14-16]}$. As the prevalence of clinical stability in the total study population is a weighted average of the prevalence of clinical stability in the de-escalation and the continuation group, the prevalence in one group can be calculated from the prevalence in the other group. We assumed a high prevalence for clinical stability in the de-escalation group, so we varied the prevalence from $80 \%$ to $100 \%$, with corresponding 
calculated prevalence's in the continued group between $80 \%$ and $75 \%$ to arrive at the overall prevalence of $80 \%$. The assumed crude odds ratio (OR) between clinical stability at 72 hours and 30-day mortality was 0.14 , based on unpublished data of a randomized controlled trial evaluating the effect of adjunct prednisone therapy versus placebo on time to clinical stability for patients with CAP (Courtesy of dr. Blum) ${ }^{[17]}$. In this trial, clinical stability was measured every 12 hours during hospital stay and was defined as time (days) until stable normalized vital signs for $\geq 24$ hours: temperature $\leq 37.8^{\circ} \mathrm{C}$ without antipyretic agents, heart rate $\leq 100$ beats per minute, spontaneous respiratory rate $\leq$ 24 per minute, systolic blood pressure $\geq 90 \mathrm{mmHg}(\geq 100 \mathrm{mmHg}$ for patients diagnosed with hypertension) without vasopressor support, mental status back to level before CAP, oxygenation on room air or oxygen therapy $(\mathrm{PaO} 2 \geq 60 \mathrm{mmHg}$ or pulse oximetry $\geq$ $90 \%$, or $\mathrm{PaO} 2$ or pulse oximetry measurement back to baseline for patients with chronic hypoxemia or chronic oxygen therapy) ${ }^{[17]}$. To simulate the confounder of clinical stability at 72 hours in our dataset, we randomly assigned the presence and the absence of clinical stability such that the aforementioned assumptions about the three parameters were met. Subsequently, the HR of de-escalation on mortality adjusted for clinical stability was determined by including clinical stability as an extra covariate in the propensity score adjusted model. The robustness of the resulting adjusted HRs was tested by repeating the random assignment three times with a different random seed, which verified that the same adjusted HRs was achieved. In the end we plotted the crude and adjusted HR without clinical stability and the resulting HRs for different prevalence's of clinical stability.

We also quantified the strength of each confounder as the change in HR of the model with or without each confounder. For the simulated confounder (clinical stability) we used the corresponding adjusted HR when added to the model with prevalence's of resp. $90 \%$ and $100 \%$ in the de-escalation group. Data analysis was performed using SPSS for Windows, v.25.0 (SPSS, Chicago, IL, USA) and R v.3.4.3 http://www.R-projects.org/.

\section{RESULTS}

\section{Association between de-escalation and mortality}

The study cohort consisted of 3,243 patients admitted with a clinical suspicion of pneumonia. After applying the in- and exclusion criteria 1,536 patients were included for analysis (Figure 2). Empirical treatment was rank-1 in 211 (13.7\%), rank-2 in 624 (40.6\%), and rank-3 in 701 (45.6\%) patients. De-escalation occurred in 257 patients (16.7\%) and escalation occurred in $123(8.0 \%)$ patients. Most patients (1156, 75.3\%) continued 
treatment without a change in rank of antimicrobial therapy during admission (Figure 3). Median time to de-escalation was 3.0 days (IQR 2.0 - 4.0 days). Compared to patients with continued (no de-escalation) regimens, patients with de-escalation less often were current smokers $(21 / 257(8.2 \%)$ vs. 148/1068 (13.9\%), more often had a pathogen identified $(107 / 257(41.6 \%)$ vs. $303 / 1068(28.4 \%)$ and had a higher median PSI-score (103 vs. 99) (Table 2). Patients in rank 2 de-escalated less often than patients in rank $316.7 \%$ vs. 30.1\%; $p$ <0.001). Of the 257 patients with de-escalated therapy, therapy was later escalated in 14 patients (5.5\%; $0.9 \%$ of all included patients) during admission.

Crude 30-day mortality was 3.5\% (9/257) and 10.9\% (107/986) in the de-escalation and continuation groups, respectively. The crude and adjusted hazard ratios for deescalation, compared to continuation, were $0.40(95 \% \mathrm{Cl}: 0.20-0.80)$ and 0.39 (95\% Cl: $0.19-0.79)$ for day-30 mortality. The AUC of the propensity score was $0.76(95 \% \mathrm{Cl}$ : $0.73-0.79)$ and was considered acceptable.

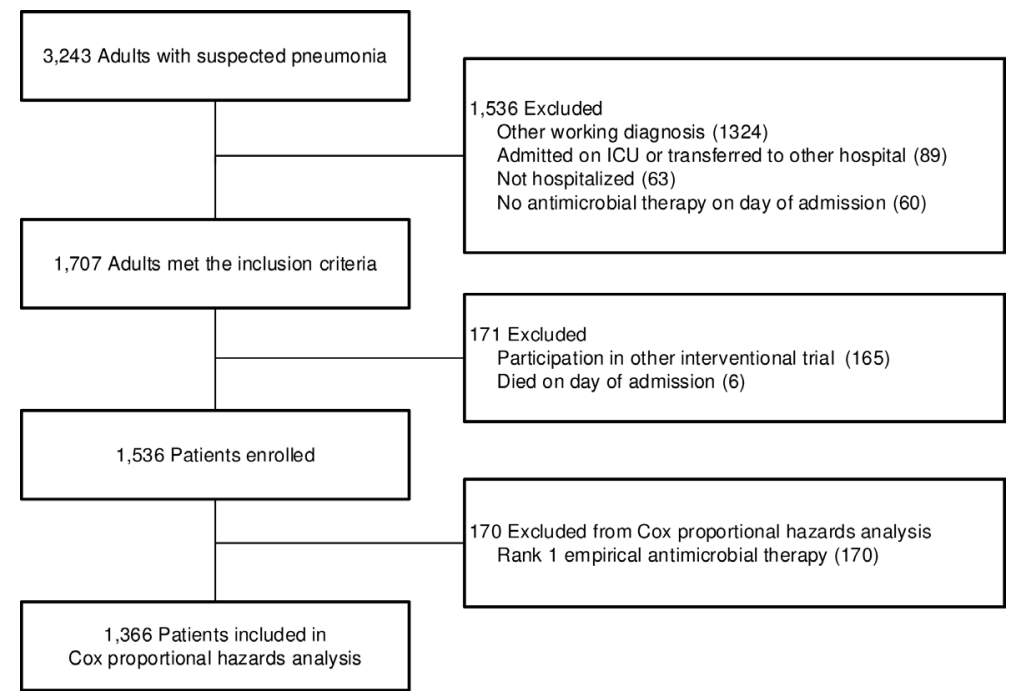

Figure 2 Patient selection. 


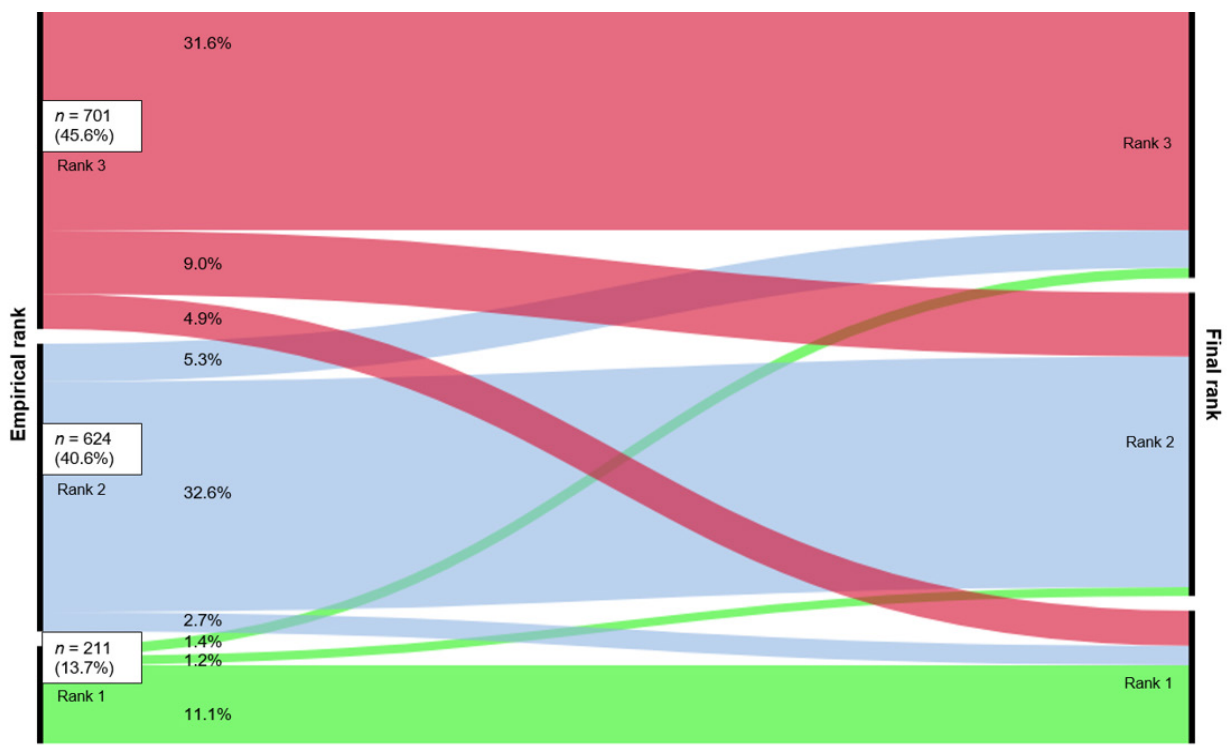

Figure 3 Alluvial-diagram of adjustment of empirical therapy. Rank-1 = narrow-spectrum- antibiotics, rank-2 = broad-spectrum-antibiotics, rank-3 = extended-spectrum-antibiotics. Continued regimen is a straight line, de-escalation is a falling line, escalation is a rising line.

Table 2. Baseline characteristics.

\begin{tabular}{lllll}
\hline & Total cohort & De-escalation & No de-escalation (rank2-3) & No de-escalation (rank 1) $^{\text {a }}$ \\
\hline Patients (N, \%) & $1536(100)$ & $257(16.7)$ & $1068(69.5)$ & $211(13.7)$ \\
Age (y, median, range) & $77(65-100)$ & $77(66-95)$ & $77(65-99)$ & $78(66-100)$ \\
Male gender (n, \%) & $1093(71.2)$ & $189(73.5)$ & $768(71.9)$ & $136(64.5)$ \\
Smoker (n, \%) & $194(12.6)$ & $21(8.2)$ & $148(13.9)$ & $25(11.8)$ \\
Co-morbidities (n, \%) & & & & $110(52.1)$ \\
$\quad$ Chronic pulmonary disease & $849(55.3)$ & $131(51.0)$ & $608(56.9)$ & $81(38.4)$ \\
$\quad$ Chronic cardiovascular disease & $650(42.3)$ & $123(47.9)$ & $446(41.8)$ & $3(1.4)$ \\
$\quad$ Chronic renal disease & $11(0.7)$ & $3(1.2)$ & $5(0.5)$ & $5(1.9)$ \\
$\quad$ Chronic liver disease & $17(1.1)$ & $5(1.9)$ & $8(0.7)$ & $54(20.6)$ \\
$\quad$ Diabetes mellitus & $322(21.0)$ & $53(20.6)$ & $215(20.1)$ & $92(80-111)$ \\
PSI score (median, IQR) & $99(82-117)$ & $103(84-121)$ & $99(82-118)$ & $44(20.9)$ \\
Antibiotic use before admission (n, \%) & $493(32.1)$ & $84(32.7)$ & $365(34.2)$ & $59(28.0)$ \\
Pathogen identified (n, \%) & $469(30.5)$ & $107(41.6)$ & $303(28.4)$ & $59(28.0)$ \\
Day of admission (n, \%) & & & & \\
$\quad$ Weekend & $613(39.9)$ & $71(27.6)$ & $263(24.6)$ & $211(100)$ \\
Empirical rank on day 1 (n, \%) & & & & NA \\
$\quad$ Rank 1 & $211(13.7)$ & NA & NA & NA \\
$\quad$ Rank 2 & $624(40.6)$ & $42(16.3)$ & $582(54.5)$ & \\
$\quad$ Rank 3 & $701(45.6)$ & $215(83.7)$ & $486(45.5)$ & \\
\hline
\end{tabular}

a Patients with a continued regimen and patients with an escalation. 


\section{Effect of confounding by indication due to clinical stability}

The results of the simulation analysis are depicted in Figure 4. Not using clinical stability for adjustment yields the afore-mentioned HR of 0.39 . When using the assumed odds ratio between clinical stability at 72 hours and 30-day mortality of 0.14 , the adjusted $\mathrm{HR}$ for de-escalation gradually increased to 1.04 with an increasing prevalence of clinical stability in patients with de-escalation up to $100 \%$. The upper boundary of $95 \%$ confidence interval crosses 1 if the prevalence of clinical stability in the de-escalated patients was $>=87 \%$. Determination of the strength of the simulated confounder, clinical stability, revealed that it was substantially stronger than any of the observed confounders in our dataset (Table 3).

Table 3 Strength of known and simulated confounders to the crude HR for 30-day mortality.

\begin{tabular}{|c|c|}
\hline Confounder & $\%$ change of crude $H R$ \\
\hline Smoking & +1.5 \\
\hline Renal disease & +1.7 \\
\hline Respiratory rate & -1.9 \\
\hline Nursing home resident & +2.0 \\
\hline Congestive heart failure & -2.2 \\
\hline Liver disease & -2.3 \\
\hline Heart rate & -2.4 \\
\hline $\mathrm{pH}$ & -2.8 \\
\hline Propensity score & -3.9 \\
\hline Partial pressure of oxygen & -4.2 \\
\hline Blood urea nitrogen & -6.5 \\
\hline Neoplastic disease & +7.4 \\
\hline Rank on day 1 & -11.4 \\
\hline \multicolumn{2}{|l|}{ Clinical stability (simulated) } \\
\hline With prevalence in de-escalated group of $90 \%$ & +37.4 \\
\hline With prevalence in de-escalated group of $100 \%$ & +157.8 \\
\hline
\end{tabular}

Variables with a change less than 1.5\%: diabetes mellitus, sodium, systolic blood pressure, hematocrit, cerebrovascular disease, antibiotic use before admission, day of admission, glucose, chronic pulmonary disease, pleural effusion, altered mental status, age, culture results, season of admission, temperature and gender. 


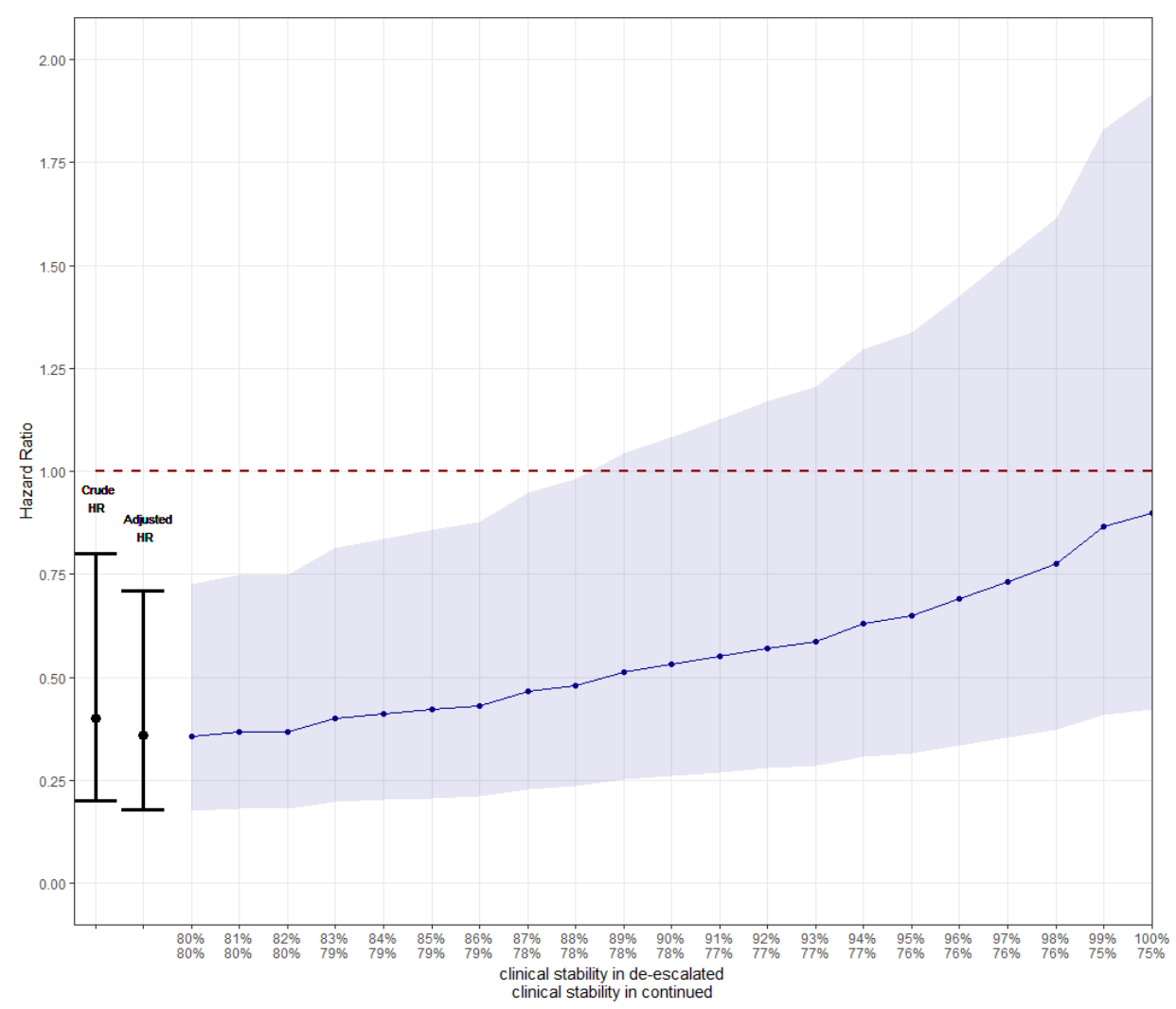

Figure 4 Effect of simulated confounder (clinical stability at 72 hours) on 30-day mortality.

The line reflects the Hazard Ratios for 30-day mortality (based on Cox proportional hazard regression analysis adjusted with propensity scores) with $95 \%$ Confidence Interval (shaded area) for different prevalence's of clinical stability in patients with and without de-escalation (horizontal axis). At the left side the weighted average of the two proportions is fixed at $80 \%$, which reflects the adjusted Hazard Ratio without adjustment for clinical stability. The dashed line represents a HR of 1. The HR rises from 0.39 to 1.04 when the prevalence of clinical stability increases to $100 \%$ in the de-escalated group. From a prevalence of clinical stability of $87 \%$ and above in the de-escalated group the upper limit of the $95 \%$ confidence interval included 1 . For example a prevalence of $90 \%$ in deescalated results in an adjusted $\mathrm{HR}$ of $0.56(95 \% \mathrm{Cl}: 0.27-1.12)$ and a prevalence of $100 \%$ results in a $\mathrm{HR}$ of $1.04(95 \% \mathrm{Cl}: 0.49-2.23)$.

\section{DISCUSSION}

In this observational study of patients hospitalized with CAP, after adjustment for observed baseline confounders de-escalation of antimicrobial therapy was associated with a $61 \%$ lower hazard of day-30 mortality. However, our simulations have demonstrated that clinical stability at 72 hours, which was not measured in our study, could fully explain this effect under reasonable, literature based assumptions. Based on these findings we conclude that the effects of de-escalation on patient outcome cannot be reliably quantified without adjustment for clinical stability and that the true effect of de- 
escalation on mortality needs to be quantified by a well-designed prospective study.

De-escalation occurred in $16.7 \%$ of the patients. During the enrolment period of our study antibiotic stewardship was not yet well established. Therefore, we expect the proportion of de-escalation in current practice to be larger. In our population, most patients continued the antibiotic regimen, even though the majority should be clinically stable based on data from the literature. In the absence of antibiotic stewardship, physicians might be more inclined to continue the regimen when it appears to be effective.

In a systematic review including different infectious diseases, de-escalation of empirical antimicrobial therapy was associated with a large reduction in mortality ${ }^{[2]}$. Although our study only included CAP patients, we expect that the mechanism of bias applies to all infectious diseases for which empirical broad-spectrum antibiotic treatment is common practice. This bias, introduced by not including clinical stability during admission, applies to all previous studies evaluating de-escalation in patients with CAP hospitalized at a non-ICU ward ${ }^{[18-22]}$. To the best of our knowledge, there are four observational studies on the association between de-escalation and mortality that adjusted for clinical stability or a similar time-varying confounder. In the first study by Joung et al. patients with intensive care unit-acquired pneumonia were included and clinical stability during admission was measured as two scores; APACHE-II (Acute Physiology and Chronic Health Evaluation II) and modified CPIS (clinical pulmonary infection score) both measured on day 5 after development of pneumonia. Both high APACHE II score $(\geq 24)$ on day 5 and a high CPIS $(\geq 10)$ on day 5 were associated with an increased 30-day pneumonia-related mortality. By including these confounders, next to other baseline covariates into the multivariable analysis the association between no de-escalation of antibiotics and 30-day mortality resulted in an aHR of $3.988(95 \% \mathrm{Cl} 0.047-6.985)^{[23]}$. The study objective was to determine independent risk factors for mortality, hence the focus of model building was not on selecting appropriate confounders and one should be careful to interpret the results as a causal effect. In the second study by Garnacho-Montero et al. patients admitted to the ICU with severe sepsis or septic shock were included and clinical stability during admission was measured as Sequential Organ Failure Assessment (SOFA) score on the day when culture results were available. A high SOFA score at culture result day was associated with a higher in-hospital mortality. When including this covariate next to other covariates the association between de-escalation and in-hospital mortality resulted in an aOR of $0.55(95 \% \mathrm{Cl} 0.32-0.98, p=0.022)^{[5]}$. In the third study by Montravers et al. patients admitted with health care-associated intra-abdominal infection admitted to ICU were included and clinical stability during admission was measured by SOFA score. Here a decreased SOFA score at day three after initiation of empirical antimicrobial 
therapy was associated with a lower 28-day mortality. By including this covariate next to other covariates in the analysis this resulted in an aHR of $0.566(95 \% \mathrm{Cl} 0.2503-$ 1.278, $p=0.171$ ) for association between de-escalation and 28-day mortality. However, this multivariate analysis also had the purpose to identify risk factors for 28-day mortality, not on selecting appropriate confounders ${ }^{[24]}$. The fourth study by Lee et al. included patients with community-onset monomicrobial Escherichia coli, Klebsiella species and Proteus mirabilis bacteremia treated empirically with broad-spectrum beta-lactams and clinical stability during admission was measured by the Pitt bacteremia score. A high Pitt bacteremia score $(\geq 4)$ at day three was associated with 4 -week mortality. After propensity score matching there was no statistically significant difference in mortality rates between de-escalation and no-switch regarding 2-week, 4-week and 8-week mortality ${ }^{[25]}$. Comparison of the studies is difficult because different criteria for deescalation and different definitions of disease severity during admission were used, and different populations were studied. The first three studies included ICU patients, and in this setting registering scores representing clinical stability is part of routine care, which makes it more feasible to include such parameters in observational studies. Although the definition for clinical stability for CAP as provided by Halm et al. ${ }^{[13]}$ is widely accepted, in clinical practice patients can be declared stable based on other criteria (e.g. feeling well, eating and drinking) even if they do not meet the formal criteria. A critique of the aforementioned studies is that all used de-escalation as a fixed variable. However, deescalation is performed on a different day for each individual and should be analyzed as a time-dependent variable, otherwise it introduces immortal time bias ${ }^{[26]}$.

It is recommended to include sensitivity analyses to estimate the potential impact of unmeasured confounding in every non-randomized study on causal associations [27]. However, for observational studies evaluating de-escalation of antimicrobial therapy this has never been done before. To strengthen our sensitivity analysis we based our assumptions about the prevalence of clinical stability and association with mortality on existing high-quality data. We further assumed that physicians will only de-escalate when a patient is clinically stable or to initiate targeted treatment for an identified pathogen. In the latter case, we still expect that most patients in whom the physician decides to de-escalate will be clinically stable. We, therefore, expect that at least $90 \%$ and probably close to $100 \%$ of de-escalated patients will be clinically stable on day three.

Strengths of our study include the pragmatic approach of using prospectively collected data of a large patient population treated with empiric antibiotics and a working diagnosis of CAP. This included patients without an identified pathogen, which increases the generalizability of our study results. The effect of de-escalation on mortality may be 
different from one country to another, or even between hospitals within one country, depending on local antibiotic practices. However, we think that the confounding effect of clinical stability is generalizable to other countries and also applies to other severe bacterial infections, because clinical stability will always be a major determinant of de-escalation. A limitation of our study is that we had to exclude 165 patients due to participation in a concurrent trial which could result in selection bias. However this was a small number of patients and participation was hospital dependent, so the influence of selection bias will be small. Another limitation of our study was that we had to make assumptions for the prevalence of clinical stability in the de-escalated and continued group and for the association between clinical stability and day-30 mortality. These were derived from different study populations, all representing CAP patients hospitalized to a non-ICU ward. Our findings suggest that adjustment for clinical stability will result in a non-significant effect of de-escalation on mortality, which would be biologically plausible. Our findings also demonstrate that the individual baseline confounders, as measured in our study, are poorly predictive for de-escalation, indicating that their correlation with clinical stability is probably also weak.

Another simplification in our analysis was that we modelled clinical stability as a binary variable on day three, which does not well represent reality. For future studies we recommend to measure clinical stability repeatedly over time, as a time-varying confounder and on a continuous scale. Finally, we did not have information on quality of our sputum samples on which the pathogen was identified. Quality of sputum samples is also a prognostic factor for de-escalation of empirical antimicrobial therapy, however we could not correct for this in our model.

The results of our analysis may also suggest that possibility of clinically relevant harm due to de-escalation cannot be excluded, as the upper boundary of the $95 \%$ confidence interval for the HR was over to 2 in the most extreme scenario. The scientific evidence for safety of de-escalation is de facto based on two RCTs. However, both RCTs are not powered for mortality. The first prospective, open-label, randomized clinical trial included patients with hospital-acquired pneumonia in an ICU without inclusion criteria regarding baseline clinical stability. After randomization de-escalation was performed three to five days after initiation if empirical treatment when culture results were available. For the association between de-escalation and 14-day mortality the RR was 0.67 (95\% $\mathrm{Cl} 0.31-1.43$ ), for 28 day mortality the RR was $0.75(95 \% \mathrm{Cl} 0.46-1.23)$ and for in-hospital mortality the RR was 0.64 (95\% Cl $0.37-1.13$ ), (calculated by the authors based on the data reported in ${ }^{[3]}$. The other multicentre non-blinded randomized non-inferiority trial evaluated the safety of de-escalation with 90-day mortality as secondary outcome in patients with severe sepsis 
admitted to an ICU without inclusion criteria regarding baseline clinical stability. After randomization de-escalation was performed after culture results were available (IQR 2-4 days after initiation of empirical therapy). In the de-escalation group 18 of 59 patients (31\%) died within 90-days, compared to 13 of 57 patients (23\%) in the continuation group, yielding an adjusted HR of $1.7(95 \% \mathrm{Cl} 0.79-3.49, \mathrm{p}=0.18)$. Although not statistically significant, this trend may indicate potential harm rather than improved outcome due to de-escalation ${ }^{[4]}$. As we have demonstrated, observational studies performed so far do not contribute to determining the safety of de-escalation because the amount of confounding by indication due to clinical stability is insurmountable. As appropriate adjustment of confounding by indication was not performed in the majority of the published observational studies on de-escalation, the ones that adjusted for clinical stability had other important limitations, and only two small RCTs have been performed, we conclude that the safety of this widely propagated antibiotic stewardship intervention should be studied more appropriately. We recommend that future observational studies addressing this research question include clinical stability in the analysis, preferably as a time-varying variable because clinical stability may change over time. It has been suggested that in the case of time-varying confounders a marginal structural model is appropriate ${ }^{[28]}$. Ultimately, although more expensive, de-escalation would be optimally studied in a pragmatic randomized controlled trial.

To conclude, the previously observed protective effect of de-escalation on mortality is likely due to confounding by unobserved factors such as clinical stability during admission. This study suggests the effect of de-escalation on mortality needs further prospective research to determine effect size more accurately.

\section{List of abbreviations}

APACHE-II, Acute Physiology and Chronic Health Evaluation-II; CAPiTA, CommunityAcquired Pneumonia immunization Trial in Adults; CPIS, clinical pulmonary infection score; OR, odds ratio; PSI, Pneumonia Severity Index; SOFA, Sequential Organ Failure Assessment.

\section{Acknowledgements}

We gratefully acknowledge dr. Claudine Blum (Medizinische Universitätsklinik, Kantonsspital Aarau, Switzerland) and colleagues for sharing unpublished data and Lufang Zhang (University Medical Centre Utrecht, the Netherlands) for assistance with the data analysis. 


\section{Conflict of interests}

No conflict of interest to disclose. 


\section{REFERENCES}

1. Davey P, Marwick CA, Scott CL, Charani E, McNeil K, Brown E, et al. Interventions to improve antibiotic prescribing practices for hospital inpatients. Cochrane Database Syst Rev. 2017;2:CD003543. Epub 2017/02/09. doi: 10.1002/14651858.CD003543.pub4. PubMed PMID: 28178770; PubMed Central PMCID: PMCPMC6464541.

2. Schuts EC, Hulscher MEJL, Mouton JW, Verduin CM, Stuart JWTC, Overdiek HWPM, et al. Current evidence on hospital antimicrobial stewardship objectives: a systematic review and meta-analysis. The Lancet Infectious Diseases. 2016;16(7):847-56. doi: 10.1016/s1473-3099(16)00065-7.

3. Kim JW, Chung J, Choi SH, Jang HJ, Hong SB, Lim CM, et al. Early use of imipenem/cilastatin and vancomycin followed by de-escalation versus conventional antimicrobials without de-escalation for patients with hospital-acquired pneumonia in a medical ICU: a randomized clinical trial. Crit Care. 2012;16(1):R28. Epub 2012/02/18. doi: 10.1186/cc11197. PubMed PMID: 22336530; PubMed Central PMCID: PMCPMC3396273.

4. Leone M, Bechis C, Baumstarck K, Lefrant JY, Albanese J, Jaber S, et al. De-escalation versus continuation of empirical antimicrobial treatment in severe sepsis: a multicenter non-blinded randomized noninferiority trial. Intensive Care Med. 2014;40(10):1399-408. Epub 2014/08/06. doi: 10.1007/s00134-014-3411-8. PubMed PMID: 25091790.

5. Garnacho Montero J, Gutiérrez Pizarraya A, Escoresca Ortega A, Corcia Palomo Y, Fernández Delgado E, Herrera Melero I, et al. De-escalation of empirical therapy is associated with lower mortality in patients with severe sepsis and septic shock. Intensive care medicine. 2014;40(1):32-40. doi: 10.1007/s00134-013-3077-7.

6. Bonten MJM, Huijts S, Bolkenbaas M, Webber C, Patterson S, Gault S, et al. Polysaccharide conjugate vaccine against pneumococcal pneumonia in adults. The New England journal of medicine. 2015;372(12):1114-25. doi: 10.1056/NEJMoa1408544.

7. Postma D, van Werkhoven C, van Elden LJR, Thijsen SFT, Hoepelman AIM, Kluytmans JAJW, et al. Antibiotic treatment strategies for community-acquired pneumonia in adults. The New England journal of medicine. 2015;372(14):1312-23. doi: 10.1056/NEJMoa1406330.

8. Wiersinga WJ, Bonten MJ, Boersma WG, Jonkers RE, Aleva RM, Kullberg BJ, et al. Management of communityacquired pneumonia in adults: 2016 guideline update from the Dutch Working Party on Antibiotic Policy (SWAB) and Dutch Association of Chest Physicians (NVALT). Netherlands journal of medicine. 2018;76(1):4-13.

9. Danish Society of Infectious Diseases. Behandling af samfundserhvervet pneumoni 2011 [August 13, 2019]. Available from: http://www.infmed.dk/guidelines.

10. Spindler C, Stralin K, Eriksson L, Hjerdt-Goscinski G, Holmberg H, Lidman C, et al. Swedish guidelines on the management of community-acquired pneumonia in immunocompetent adults--Swedish Society of Infectious Diseases 2012. Scandinavian journal of infectious diseases. 2012;44(12):885-902. Epub 2012/07/27. doi: 10.3109/00365548.2012.700120. PubMed PMID: 22830356.

11. SWAB (The Dutch Working Party on Antibiotic Policy). A-teams Praktijkgids Antimicrobial Stewardship in Nederland. 2 ed. Leusden: Buro Hans; 2018. 21-2 p.

12. Niederman MS, Mandell LA, Anzueto A, Bass JB, Broughton WA, Campbell GD, et al. Guidelines for the 
management of adults with community-acquired pneumonia. Diagnosis, assessment of severity, antimicrobial therapy, and prevention. Am J Respir Crit Care Med. 2001;163(7):1730-54. Epub 2001/06/13. doi: 10.1164/ ajrccm.163.7.at1010. PubMed PMID: 11401897.

13. Halm EA, Fine MJ, Marrie TJ, Coley CM, Kapoor WN, Obrosky DS, et al. Time to clinical stability in patients hospitalized with community-acquired pneumonia: implications for practice guidelines. Jama. 1998;279(18):1452-7. Epub 1998/05/26. doi: 10.1001/jama.279.18.1452. PubMed PMID: 9600479.

14. Castro-Guardiola A, Viejo-Rodriguez AL, Soler-Simon S, Armengou-Arxe A, Bisbe-Company V, PenarrojaMatutano G, et al. Efficacy and safety of oral and early-switch therapy for community-acquired pneumonia: a randomized controlled trial. Am J Med. 2001;111(5):367-74. Epub 2001/10/05. doi: 10.1016/s00029343(01)00868-3. PubMed PMID: 11583639.

15. Kohno S, Yanagihara K, Yamamoto Y, Tokimatsu I, Hiramatsu K, Higa F, et al. Early switch therapy from intravenous sulbactam/ampicillin to oral garenoxacin in patients with community-acquired pneumonia: a multicenter, randomized study in Japan. Journal of infection and chemotherapy : official journal of the Japan Society of Chemotherapy. 2013;19(6):1035-41. Epub 2013/05/23. doi: 10.1007/s10156-013-0618-5. PubMed PMID: 23695232

16. Oosterheert JJ, Bonten MJ, Schneider MM, Buskens E, Lammers JW, Hustinx WM, et al. Effectiveness of early switch from intravenous to oral antibiotics in severe community acquired pneumonia: multicentre randomised trial. BMJ (Clinical research ed). 2006;333(7580):1193. Epub 2006/11/09. doi: 10.1136/bmj.38993.560984.BE. PubMed PMID: 17090560; PubMed Central PMCID: PMCPMC1693658.

17. Blum CA, Nigro N, Briel M, Schuetz P, Ullmer E, Suter-Widmer I, et al. Adjunct prednisone therapy for patients with community-acquired pneumonia: a multicentre, double-blind, randomised, placebo-controlled trial. Lancet (London, England). 2015;385(9977):1511-8. Epub 2015/01/23. doi: 10.1016/s0140-6736(14)62447-8. PubMed PMID: 25608756

18. Carugati M, Franzetti F, Wiemken T, Kelley RR, Peyrani P, Blasi F, et al. De-escalation therapy among bacteraemic patients with community-acquired pneumonia. Clin Microbiol Infect. 2015;21(10):936 e11-8 Epub 2015/06/28. doi: 10.1016/j.cmi.2015.06.015. PubMed PMID: 26115864

19. Cremers AJ, Sprong T, Schouten JA, Walraven G, Hermans PW, Meis JF, et al. Effect of antibiotic streamlining on patient outcome in pneumococcal bacteraemia. J Antimicrob Chemother. 2014;69(8):2258-64. Epub 2014/04/15. doi: 10.1093/jac/dku109. PubMed PMID: 24729585.

20. Viasus D, Simonetti AF, Garcia-Vidal C, Niubo J, Dorca J, Carratala J. Impact of antibiotic de-escalation on clinical outcomes in community-acquired pneumococcal pneumonia. J Antimicrob Chemother. 2017;72(2):547-53. Epub 2016/11/01. doi: 10.1093/jac/dkw441. PubMed PMID: 27798219.

21. West DM, McCauley LM, Sorensen JS, Jephson AR, Dean NC. Pneumococcal urinary antigen test use in diagnosis and treatment of pneumonia in seven Utah hospitals. ERJ Open Res. 2016;2(4). Epub 2017/01/06. doi: 10.1183/23120541.00011-2016. PubMed PMID: 28053969; PubMed Central PMCID: PMCPMC5152836.

22. Yamana H, Matsui H, Tagami T, Hirashima J, Fushimi K, Yasunaga H. De-escalation versus continuation of empirical antimicrobial therapy in community-acquired pneumonia. J Infect. 2016;73(4):314-25. Epub 2016/07/11. doi: 10.1016/j.jinf.2016.07.001. PubMed PMID: 27394401. 
23. Joung MK, Lee JA, Moon SY, Cheong HS, Joo EJ, Ha YE, et al. Impact of de-escalation therapy on clinical outcomes for intensive care unit-acquired pneumonia. Crit Care. 2011;15(2):R79. Epub 2011/03/04. doi: 10.1186/cc10072. PubMed PMID: 21366903; PubMed Central PMCID: PMCPMC3219332.

24. Montravers P, Augustin P, Grall N, Desmard M, Allou N, Marmuse JP, et al. Characteristics and outcomes of anti-infective de-escalation during health care-associated intra-abdominal infections. Crit Care. 2016;20:83. Epub 2016/04/08. doi: 10.1186/s13054-016-1267-8. PubMed PMID: 27052675; PubMed Central PMCID: PMCPMC 4823898.

25. Lee CC, Wang JL, Lee CH, Hung YP, Hong MY, Tang HJ, et al. Clinical benefits of antimicrobial de-escalation in adults with community-onset monomicrobial Escherichia coli, Klebsiella species and Proteus mirabilis bacteremia. Int J Antimicrob Agents. 2017;50(3):371-6. Epub 2017/07/12. doi: 10.1016/j.ijantimicag. 2017.03.024. PubMed PMID: 28694235.

26. Jones M, Fowler R. Immortal time bias in observational studies of time-to-event outcomes. J Crit Care. 2016;36:195-9. Epub 2016/11/05. doi: 10.1016/j.jcrc.2016.07.017. PubMed PMID: 27546771.

27. Groenwold RH, Nelson DB, Nichol KL, Hoes AW, Hak E. Sensitivity analyses to estimate the potential impact of unmeasured confounding in causal research. International journal of epidemiology. 2010;39(1):107-17. Epub 2009/12/02. doi: 10.1093/ije/dyp332. PubMed PMID: 19948779.

28. Hernan MA, Hernandez-Diaz S, Werler MM, Mitchell AA. Causal knowledge as a prerequisite for confounding evaluation: an application to birth defects epidemiology. Am J Epidemiol. 2002;155(2):176-84. Epub 2002/01/16. doi: 10.1093/aje/155.2.176. PubMed PMID: 11790682. 
abo

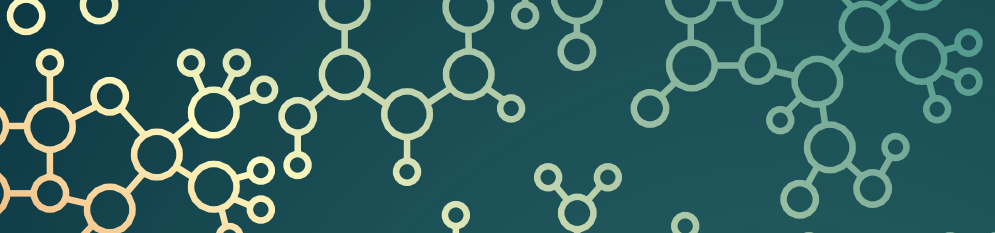
o

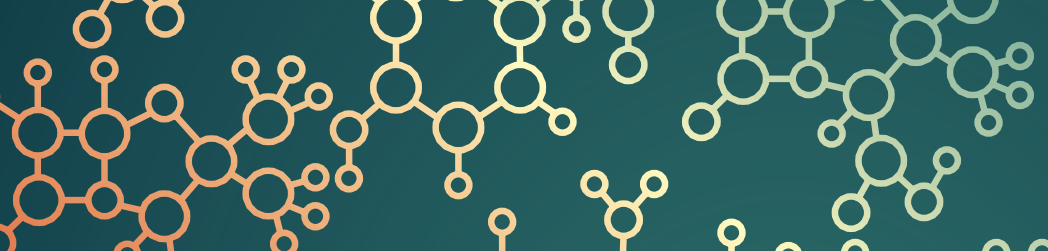

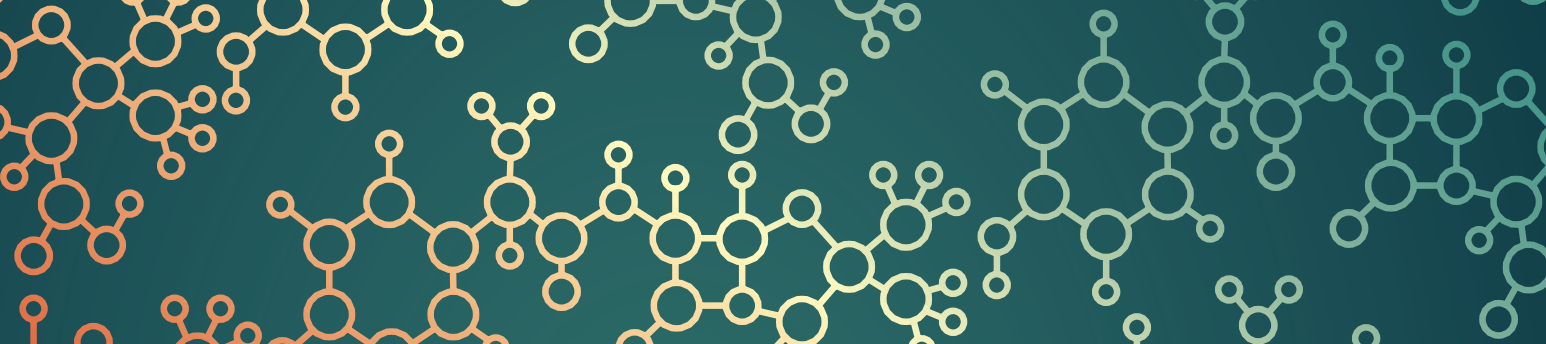

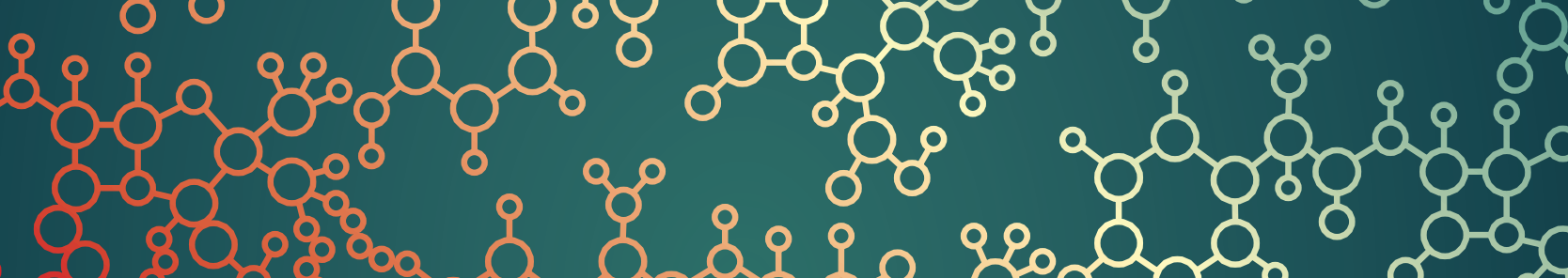
80\%

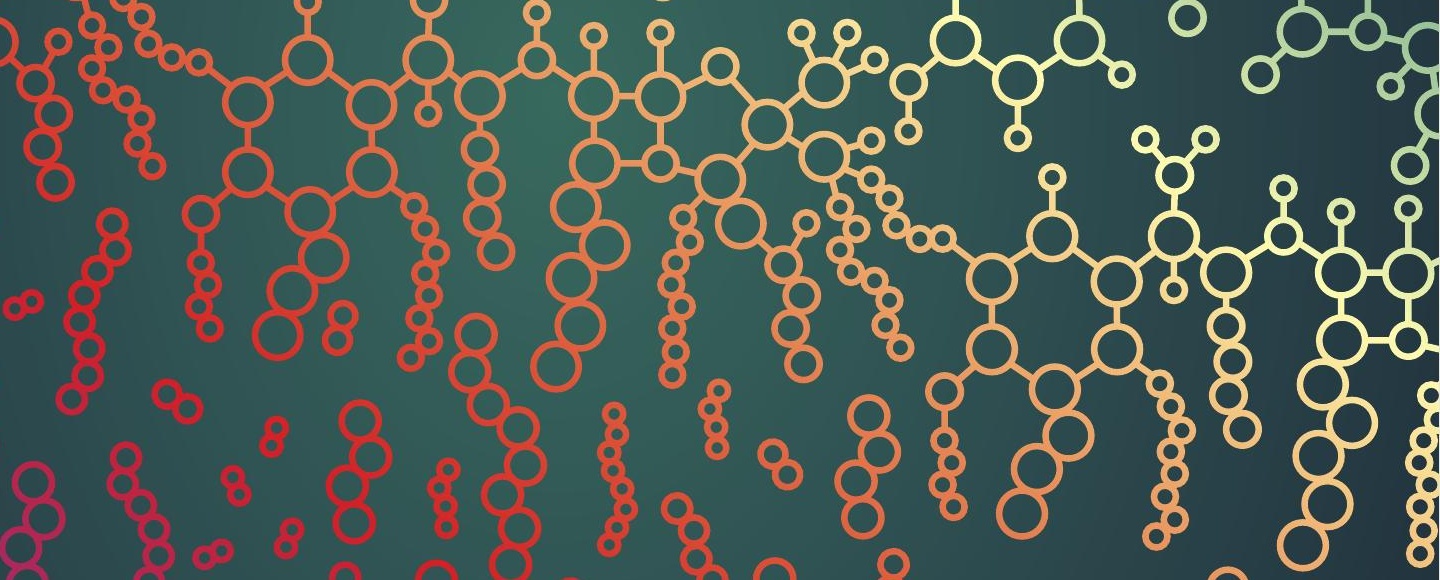
$88 \% 888$ $\circ 88$ \& 8

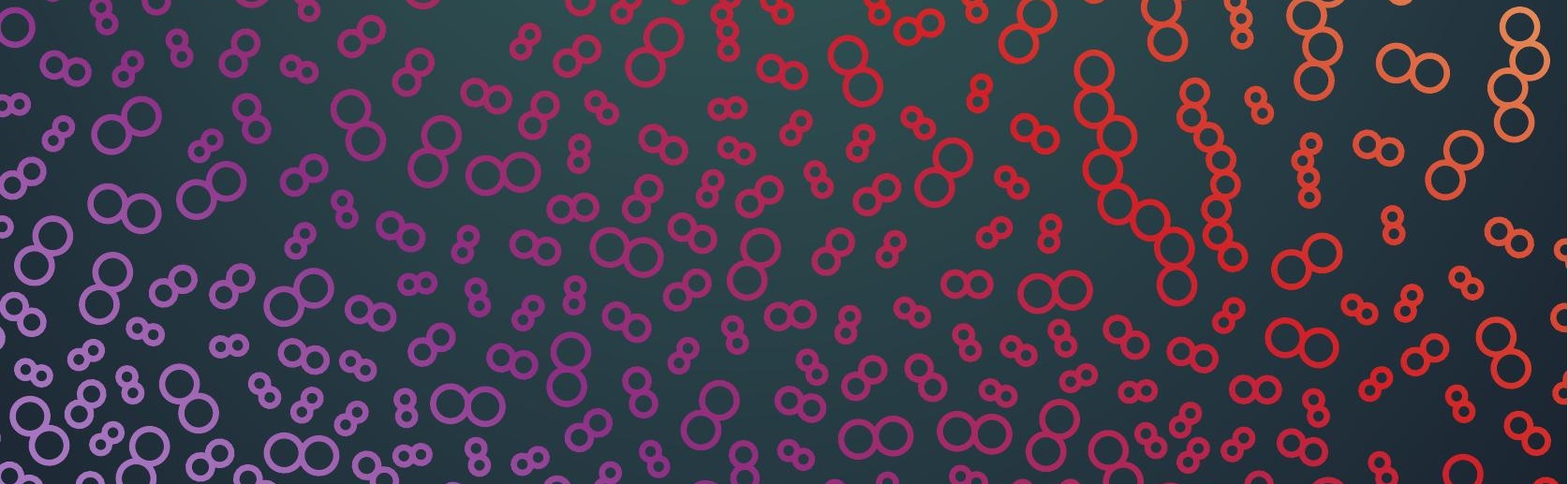




\section{Safety of de-escalation of empirical therapy in terms of mortality: a literature review of study methodology and recommendations for observational studies.}

Inger van Heijl, Valentijn A. Schweitzer, Paul D. van der Linden, Marc J.M. Bonten, Cornelis H. van Werkhoven 


\section{ABSTRACT}

\section{Introduction}

The safety of de-escalation of empirical antimicrobial therapy is largely based on observational data, with many reporting protective effects on mortality. As there is no plausible biological explanation for this phenomenon it is most probably caused by confounding by indication.

\section{Areas covered}

We evaluate the methodology used in observational studies on the effects of deescalation of antimicrobial therapy on mortality. We extended the search of a recent systematic review and identified 52 observational studies. The heterogeneity in study populations was large. Only 19 (36.5\%) studies adjusted for confounders and four (8\%) adjusted for clinical stability during admission, all as a fixed variable. All studies had methodological limitations, most importantly the lack of adjustment for clinical stability, causing bias towards a protective effect.

\section{Expert opinion/commentary}

The methodology used in studies evaluating the effects of de-escalation on mortality requires improvement. We depicted all potential confounders in a directed acyclic graph to illustrate all associations between exposure (de-escalation) and outcome (mortality). Clinical stability is an important confounder in this association and should be modeled as a time-varying variable. We recommend to include de-escalation as time-varying exposure and use inverse-probability-of-treatment weighted marginal structural models to properly adjust for time-varying confounders. 


\section{INTRODUCTION}

Empirical antimicrobial therapy of suspected bacterial infections often includes broadspectrum antibiotics to ensure coverage of multiple potential pathogens. According to antimicrobial stewardship principles empirical treatment should be de-escalated when possible to reduce antimicrobial pressure and antimicrobial resistance ${ }^{[1]}$. In a systematic review, de-escalation of empirical antimicrobial therapy compared to continuation of empirical treatment was associated with a relative risk reduction of $56 \%$ (95\% Cl 34\%70\%) for mortality, which was based on one randomized controlled trial (RCT) and 19 observational studies ${ }^{[2]}$. However the RCT, ${ }^{[3]}$ demonstrated no difference in mortality between de-escalation and continuation. A total of 116 patients with severe sepsis admitted to an ICU were included and de-escalation was not statistically significantly associated with mortality with an adjusted HR of $1.7(95 \% \mathrm{Cl} 0.79-3.49, \mathrm{p}=0.18)^{[3]}$. The trial had relatively small sample sizes resulting in imprecise effect estimates. Therefore, the evidence of safety of de-escalation is largely based on observational data reporting a reduction in mortality. We postulate that the mortality reduction based on interpretation of observational data could reflect a true causal effect if narrow-spectrum antibiotics are more effective or safer than broad-spectrum antibiotics, i.e. if they cause less (ultimately fatal) side effects. The first hypothesis (more effective) we consider unlikely because generally the antimicrobial spectrum and activity of narrow-spectrum antibiotics is entirely included in the spectrum and activity of broad-spectrum antibiotics. For specific pathogens narrow-spectrum antibiotics can potentially be more effective than broad-spectrum antibiotics, such as penicillin for Staphylococcus aureus infections although the level of evidence is low ${ }^{[5]}$ However, this is not likely to result in a mortality reduction of $56 \%$ as seen in the aforementioned systematic review. The second (less side effects) might hold for less severe outcomes such as duration of hospitalization or complications such as Clostridioides difficile infection, but is considered unlikely or of indiscernible size for mortality. If not causal, the observed mortality reduction may reflect residual confounding by indication, meaning that, even after adjustment for measured confounders, the prognosis of those in whom antimicrobial therapy is de-escalated is more favorable compared to those continuing or escalating the antibiotic treatment due to differences in unmeasured patient characteristics.

In this paper we review the methodology used in observational studies on the effect of de-escalation of empirical antimicrobial therapy on mortality, followed by an expose of causal effects that need to be taken into account in the study of de-escalation. Finally, we provide recommendations for the design and analysis of antibiotic de-escalation studies. 


\section{GENERAL CHARACTERISTICS OF OBSERVATIONAL STUDIES EVALUATING DE-ESCALATION AND MORTALITY}

A PubMed search was done until October 2019 to find all studies evaluating the effect of de-escalation of empirical antimicrobial therapy on mortality. We used the search strategy described by Schuts et al. ${ }^{[2]}$ (Appendix 1). In addition, we checked all other available systematic reviews and meta-analyses for missed publications ${ }^{[6-10]}$. We selected papers evaluating the effect of de-escalation on mortality (as primary or secondary outcome), which yielded 52 observational studies ${ }^{[11-62]}$. The heterogeneity in study populations between the studies was large (Table 1). 18 studies (34.6\%) were done in an ICU setting, the remaining in a non-ICU hospital setting.

Table 1 Study populations.

\begin{tabular}{ll}
\hline Study population & $\mathbf{N}=\mathbf{5 2}(\mathbf{\%})$ \\
\hline Community-acquired pneumonia & $5(9.6)$ \\
Hospital-acquired or ventilator-associated pneumonia & $12(23.1)$ \\
Healthcare-associated pneumonia & $1(1.9)$ \\
Pneumonia (acquisition not specified) & $4(7.7)$ \\
Urinary tract infection & $2(3.8)$ \\
Intra-abdominal infections & $1(1.9)$ \\
Skin infections & $1(1.9)$ \\
Bloodstream infections & $10(19.2)$ \\
Severe sepsis and/or septic shock & $5(9.6)$ \\
Critically ill patients & $2(3.8)$ \\
Neutropenic fever & $2(3.8)$ \\
Any infection in severe aplastic anemia patients & $1(1.9)$ \\
Any infections treated with specific antibiotic classes & $6(11.5)$ \\
\hline
\end{tabular}

A retrospective design was used in 34 (65.4\%) of the studies. The majority was performed in a single center $(n=42 ; 80.8 \%)$. Mortality differences were reported as crude estimates in $33(63.5 \%)$ studies. In the other studies multivariable analyses ( $n=19 ; 36.5 \%)$ were used to adjust for potential confounders; 11 (21.1\%) used logistic regression, 1 (1.9\%) used Poisson regression and 7 (13.5\%) used Cox proportional hazards regression. The effect estimates obtained in these multivariable analyses are depicted in Figure 1, clustered by study population. Studies that did not present an OR, HR or RR in their article $[46,51,57]$ are not included. The confidence intervals between the different studies per study population overlap largely and the point estimates are mostly in favor of de-escalation. This means that the individual studies yield comparable results. In the next section we will discuss the limitations in methodology used in observational de-escalation studies. 


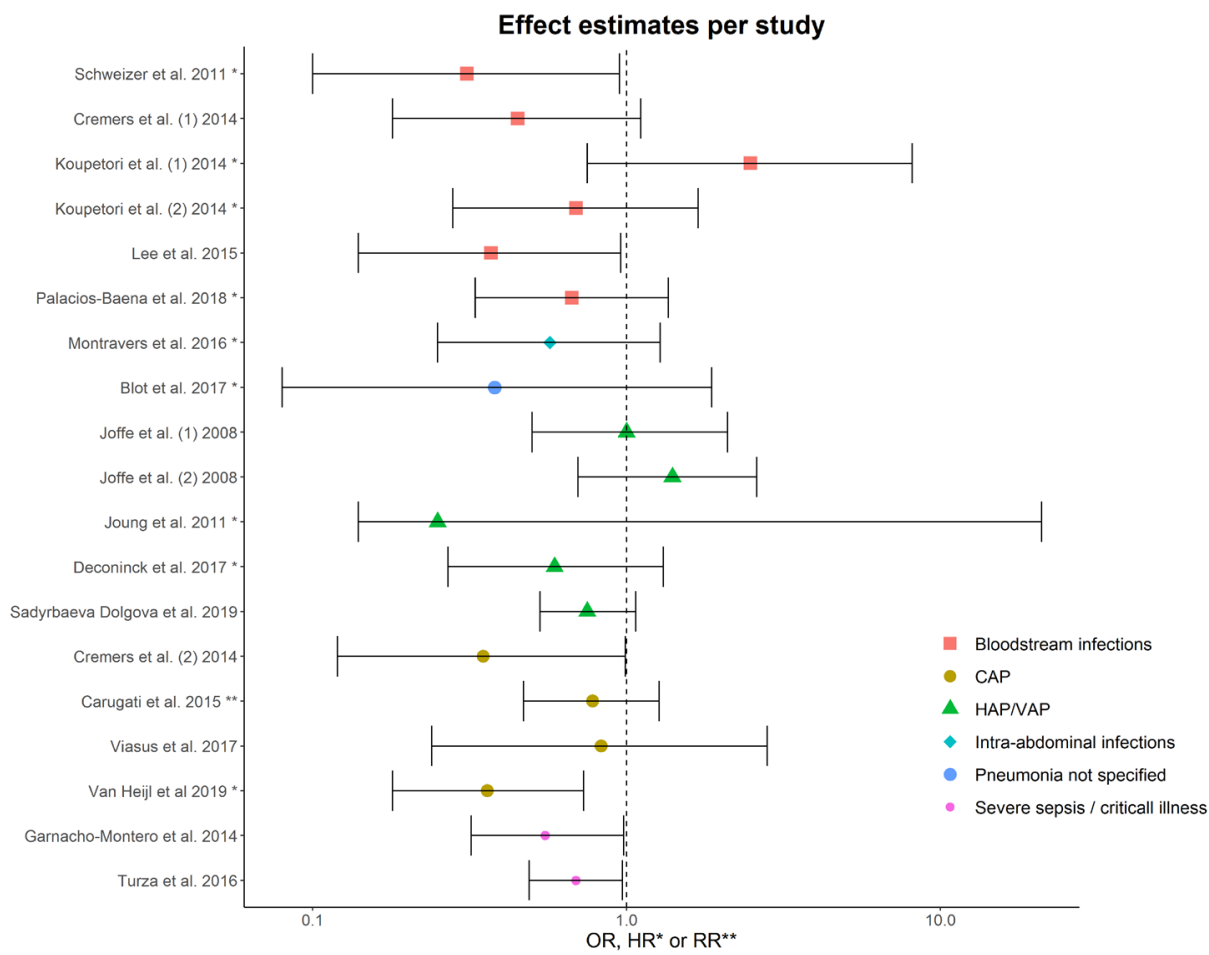

Figure 1: Adjusted effect estimates for the association between de-escalation of empirical antimicrobial therapy and mortality. Koupetori (1) and (2) are effect estimates from different time periods (resp. 2006-2009 and 2010-2013). Joffe (1) and (2) are effect estimates from culture positive and culture negative patients, respectively. Joung et al. reported an effect estimate for non-de-escalation; we calculated the inverse effect estimate for de-escalation which results in an aHR of $0.25(95 \% \mathrm{Cl} 0.14-21)$. Note that this confidence interval seems incorrect, as it is asymmetric on a log scale, which also was the case for the reported confidence interval. We contacted the corresponding author to verify this, however we received no response.

\section{METHODOLOGICAL LIMITATIONS IN DE-ESCALATION STUDIES}

Several limitations were identified in methodology of observational studies regarding the association between de-escalation of empirical antimicrobial therapy on mortality after evaluating the abovementioned studies. For 33 studies solely reporting crude estimates on mortality we confine ourselves by stating that they suffer from severe confounding by indication and are of no use for a causal inference. For the remaining we discuss studies that adjusted for potential confounders $(N=19)$ [16,21,22, 28,29,32,34,35,41,44,46-48,51,54,57,58,61,62]. For the purpose of this review we focus on three main issues: 1) lack of adjustment for the patients' clinical course, 2) modeling de-escalation as a fixed variable, and 3) modeling 
time-varying confounders as fixed variables.

All studies adjusted for baseline characteristics as potential confounders, which are measured from the time point the patient is enrolled in the study. In Table 2 we have summarized all the potential confounders used in the studies, categorized by type of baseline factor.

Table 2. Summary of all potential confounders used in individual studies.

\begin{tabular}{|c|c|c|c|}
\hline Variable group & Variables used & Frequency $(n=19)$ & Reference \\
\hline Baseline disease severity & $\begin{array}{l}\text { APACHE II score, CPIS score, SOFA score, PSI score, A-DROP, } \\
\text { Pitt bacteremia score, CPIS score, ICU admission before onset, } \\
\text { mechanical ventilation before onset, presence of sepsis or septic } \\
\text { shock. }\end{array}$ & $17(90 \%)$ & $\begin{array}{l}{[20,21,28,31} \\
33,34,40,43 \\
45-47,50,53 \\
56,57,60,61]\end{array}$ \\
\hline Comorbidities & $\begin{array}{l}\text { No. of comorbidities, Charlson index, McCabe classification, or } \\
\text { specific type of comorbidity. }\end{array}$ & $14(74 \%)$ & $\begin{array}{l}{[15,21,27,31,34} \\
43,45,46,50 \\
53,56,57,60,61]\end{array}$ \\
\hline Demographics & $\begin{array}{l}\text { Age, gender, BMI, previous use of antibiotics, previous } \\
\text { hospitalization, nursing home residence. }\end{array}$ & $12(63 \%)$ & $\begin{array}{l}{[15,21,28,40} \\
45-47,53,56 \\
57,60,61]\end{array}$ \\
\hline Antibiotic therapy & $\begin{array}{l}\text { (In)appropriateness of initial therapy, time to appropriate therapy, } \\
\text { monotherapy vs. combination therapy, specific type (or rank) of } \\
\text { antibiotic therapy. }\end{array}$ & $11(58 \%)$ & $\begin{array}{l}{[20,21,27,28} \\
33,43,45,47 \\
56,60,61]\end{array}$ \\
\hline $\begin{array}{l}\text { Hospital or department of } \\
\text { admission }\end{array}$ & Type of hospital/department. & $5(26 \%)$ & $\begin{array}{l}{[27,28,45,57,} \\
60]\end{array}$ \\
\hline $\begin{array}{l}\text { Disease severity during } \\
\text { admission }\end{array}$ & $\begin{array}{l}\text { Acute Physiology and Chronic Health Evaluation (APACHE) II score } \\
\text { at ICU day five, modified Clinical pulmonary infection score (CPIS) } \\
\text { at ICU day five, sequential organ failure assessment (SOFA) score } \\
\text { at culture result day, SOFA score at ICU day three, Pitt bacteremia } \\
\text { score (PBS) at day three after start of antibiotic treatment. }\end{array}$ & $4(21 \%)$ & {$[20,28,40,50]$} \\
\hline Laboratory results & $\begin{array}{l}\text { Creatinine, hemoglobin, } \mathrm{pH} \text {, blood urea nitrogen, sodium, } \\
\text { glucose, hematocrit, partial pressure of oxygen }\end{array}$ & $3(16 \%)$ & {$[34,46,61]$} \\
\hline Signs and symptoms & $\begin{array}{l}\text { Tachycardia, altered mental status, respiratory rate, systolic blood } \\
\text { pressure, temperature, heart rate }\end{array}$ & $2(11 \%)$ & {$[53,61]$} \\
\hline \multirow[t]{4}{*}{ Other } & Specific surgery & $1(5 \%)$ & {$[40]$} \\
\hline & Pneumococcal vaccination & $1(5 \%)$ & [53] \\
\hline & Injury Severity Score (ISS) & $1(5 \%)$ & [56] \\
\hline & Invasive procedures & $1(5 \%)$ & {$[60]$} \\
\hline
\end{tabular}

* Sorted from most common to least common used baseline factors as confounders.

Although these studies used multiple baseline characteristics as potential confounders, this is probably insufficient to adjust for confounding by indication. Our previous study 
which aimed to quantify the potential confounding effect of clinical stability during hospital stay on the estimated impact of de-escalation on mortality in patients with CAP, suggests that clinical stability in de-escalated patients is likely to explain the lower mortality observed in patients after de-escalation ${ }^{[62]}$. This was done by simulating a variable representing clinical stability on day three, using data on prevalence and associations with mortality from the literature. Therefore, it is important to not only include confounders measured at the time of admission, but also confounders that occur during hospital stay. This is intuitive because the decision to de-escalate is made several days after initiation of empirical therapy and is influenced by clinical stability during hospital stay and available culture results. As clinical stability is also a strong prognostic factor, not including this in the analysis inevitably results in biased effect estimates in favor of de-escalation. Only four studies adjusted for clinical stability or a similar variable indicating the clinical course up to the time of de-escalation ${ }^{[21,29,41,51]}$ (Table 2). Three of these studies included patients admitted to an ICU; Joung et al. included APACHE II score (used in ICU ${ }^{[63]}$ ) and modified CPIS (used for VAP ${ }^{[64]}$ ) at day five ${ }^{[21]}$. Garnacho Montero et al. included SOFA score (used in ICU ${ }^{[65]}$ ) at culture result day ${ }^{[29]}$ and Montravers included SOFA score at day three ${ }^{[41]}$. Parameters to establish clinical stability during admission are measured (and registered) more regularly in ICU than in non-ICU populations. So, it is probably more convenient to collect such data and to adjust for variables representing clinical stability during admission in ICU populations. The fourth de-escalation study that included a variable predictive for clinical course was performed in a population with and without admission at ICU, and included PBS (used in bloodstream infections ${ }^{[66]}$ ) at day three after start of antibiotic treatment ${ }^{[51]}$. Although there are specific criteria for clinical stability in patients with CAP ${ }^{[67]}$, which have been used in studies evaluating iv to oral switches ${ }^{[68,69]}$, these have not been used in de-escalation studies.

Another limitation in observational de-escalation studies is that de-escalation is analyzed as a time-fixed variable, which applies to all 19 studies that corrected for confounders. For example, in one patient de-escalation occurs at day 3 of hospital admission. Then survival time until day 3 is incorrectly counted as survival time for the de-escalation group, when in fact these patients were not exposed to de-escalation until day 3. Not adjusting for time-varying exposures results in immortal time bias (also termed timedependent bias) ${ }^{[70]}$ in favor of the de-escalated group. This can be intuitively understood as too much survival time being incorrectly classified as survival time for de-escalation. In only one study de-escalation was included as a time-varying variable in the analysis, but unfortunately without adjustment for confounders ${ }^{[33]}$.

A third common limitation is analyzing all possible confounders as time-fixed variables. 
Some confounders, such as clinical stability, differ over time with different trajectories for different patients. Such variables, when analyzed as time-fixed confounders, lead to biased estimates, as explained in the next sections. The four studies that correct for a variable indicating the clinical course up to the time of de-escalation mentioned above $[21,29,41,51]$, have included this variable in a time-varying way. For example, in the study from Montravers et al. ${ }^{[41]}$, clinical improvement is defined as a $>2$ points decrease in SOFA score on day three compared to day zero or a SOFA score of 0 points. Patients that died or were discharged alive within the first three days were excluded from the analysis. In the multivariable analysis, clinical improvement was included as a confounder. Deescalation was analyzed after 48 hours. The difference in SOFA score was assessed at day 3 , which is usually the day when empirical therapy is evaluated and when culture results are available, and most de-escalation decisions are taken. This approach adjusts for clinical improvement, however still clinical stability is reached at different points in time for individual patients so including it as a real time-varying confounder will be more appropriate. This causes collider stratification bias, i.e. adjusting for variable that may occur after escalation, which we will elaborate on in section 4.

In summary, all studies evaluating the effects of de-escalation have methodological limitations, most importantly the lack of adjustment for the clinical course, ignoring timevarying exposure, and ignoring the time-varying nature of some confounders. In the next section we provide a proposal to study the causal effect of de-escalation on mortality.

\section{EXPERT OPINION}

To study the causal effect of de-escalation on mortality we first need to consider all variables that might influence this association. We will first propose causal diagrams, also called directed acyclic graphs (DAGs) visualizing the causal associations, after which we will provide recommendations for future studies with observational data.

\section{Proposal of a DAG for studying the causal effect of de-escalation on mortality}

In observational data it is important to adjust for confounding. The preferred method to identify confounders is to use prior knowledge. In recent years DAGs have been increasingly used to identify confounders (i.e. common causes of the exposure and the outcome) ${ }^{[71,72]}$. In Figure 2 a simple DAG is depicted on the relation between exposure and outcome with one confounder. All DAGs consist of variables connected by arrows that represent direct causal effects (which can denote positive or negative associations) and they are acyclic because arrows always go in one direction and a causal path is not 
allowed to go back to its origin ${ }^{\left[{ }^{711}\right.}$. Not adjusting for confounder $\mathrm{C}$ in Figure 2, will bias the causal association between exposure $\mathrm{E}$ and outcome $\mathrm{O}$. This pathway from $\mathrm{E}$ to $\mathrm{O}$ through $\mathrm{C}$ is also called a 'backdoor path' which is a non-causal pathway. When adjusting for confounder C this 'backdoor path' is closed (and so is the non-causal pathway).

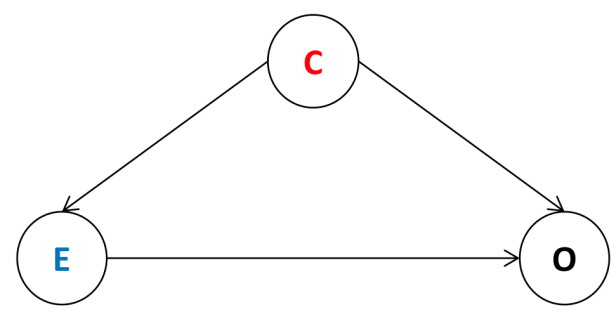

Figure 2 An example of a causal diagram (or DAG). $E=$ exposure, $\mathrm{O}=$ outcome and $\mathrm{C}=$ confounder. For example, in the relationship between alcohol consumption (E) and lung cancer (O), smoking $(C)$ is highly correlated with alcohol consumption and also a cause of lung cancer.

If both arrows from $C$ to $E$ and from $C$ to $O$ were directed to $C$, then $C$ is not a confounder but a collider (Figure 3), which is a variable caused by both exposure (E) and outcome (O). When adjusting for a collider, a 'backdoor path' (or a non-causal pathway) is introduced rather than closed, which causes bias rather than to solve it.

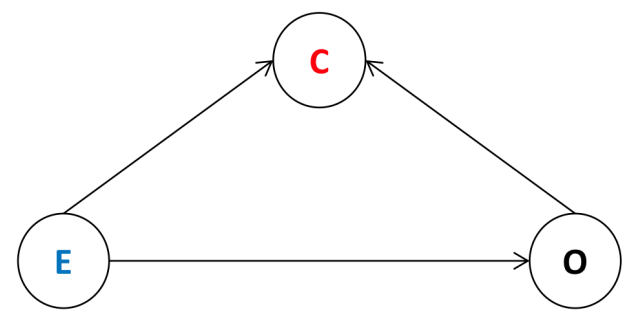

Figure 3 DAG with a collider. $E$ = exposure, $\mathrm{O}=$ outcome and $\mathrm{C}=$ collider. For example, in the relationship between obesity (E) and cardiovascular disease (O), there are also other risk factors for cardiovascular disease (C). $\mathrm{E}$ and $\mathrm{C}$ both are causes of cardiovascular disease, which makes $\mathrm{C}$ a collider.

It is therefore essential to distinguish confounders from colliders, for example with a DAG, because it is important to adjust for confounders and not for colliders. Of note, a collider can also exist through a proxy or precursor of the outcome as we will see later. Finally, in Figure 4 the effect of exposure (E) can be mediated through an intermediate 
(M) on outcome (O). Adjustment for intermediates is not necessary; there is no 'backdoor path' because $M$ is on the causal pathway of $E$ to $O$. Researcher may wish to adjust for intermediates if they are explicitly interested in the relative contribution of distinguished causal pathways, rather than estimation of the main causal effect, but this is beyond the scope of the current paper.

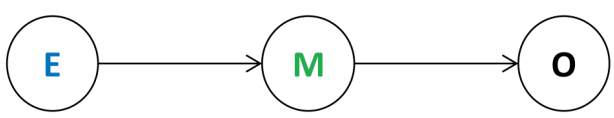

Figure 4 DAG with an intermediate. $E=$ exposure, $\mathrm{O}=$ outcome and $\mathrm{M}=$ intermediate. For example, statins $(E)$ reduce cholesterol in blood (M), and thereby lower the risk for a stroke (O).

For estimating the causal relationship between de-escalation of empirical therapy and mortality, we consider the following aspects essential: 1) the exposure de-escalation is time-varying, i.e. exposure of individual patients is set at different points in time and 2) confounders can be time-fixed or time-varying. Examples of time-fixed confounders are patients' comorbidity and severity of disease at presentation (more examples are presented in Table 2). Examples of time-varying confounders are culture results and clinical stability. After a certain time period culture results become available (pathogen detection) and clinical stability can be reached; the values of these variables change over time and influence the decision for de-escalation. In Figure 5 we present the DAG in which all important factors involved in the association of de-escalation of empirical therapy on mortality are depicted. $\mathrm{E}$ is exposure, which is the change of empirical antimicrobial therapy; either de-escalation, escalation or continuation. Exposure is time-varying presented as $E_{1}$ (exposure day 1 ) and $E_{t}$ (exposure day $t$ ), which occurs after the start of empirical therapy (A) at day of admission. Also exposure (E) influences future clinical stability (C) and is associated with outcome $\mathrm{O}$. $\mathrm{O}$ is the outcome which is mortality. The other variables in this diagram are fixed or time-varying confounders in the association between exposure $\mathrm{E}$ and outcome $\mathrm{O}$. B is a collection of time-fixed confounders, such as comorbidities and disease severity. $B$ influences empirical therapy (A), the unknown pathogen $\left(P_{0}\right)$, clinical stability at day $O\left(C_{0}\right)$ and mortality $(O)$. B is very disease specific and researchers should take efforts to identify all variables relevant to the disease and setting of investigation prior to commencing the study. Both $\mathrm{P}$ and $\mathrm{C}$ are time-varying confounders, as mentioned above, representing pathogen detection and clinical stability. $P_{0}$ is the unknown pathogen and $P_{1}$ and $P_{t}$ are day 1 and day $t$, when the culture results could be available and the pathogen is possibly detected. $P_{0}$ influences ) clinical stability 
at day $O\left(\mathrm{C}_{0}\right)$ and mortality $(\mathrm{O}) . \mathrm{P}_{1}$ and $\mathrm{P}_{\mathrm{t}}$ influence exposure $(\mathrm{E})$ by the decision to deescalate, escalate or continue treatment. $\mathrm{C}_{0}$ is clinical stability at the day of admission, $\mathrm{C}_{1}$ and $\mathrm{C}_{\mathrm{t}}$ is clinical stability determined on day 1 and day $\mathrm{t}$. Clinical stability $(\mathrm{C})$ influences mortality (O). For simplification we only used $E_{1}, P_{1}$ and $C_{1}$ measured at day 1 , all other admission days are presented as $E_{t^{\prime}} P_{t}$ and $C_{t}$. There is one important issue to add to the already complicated causal relationship between de-escalation (E) and mortality (O), which is clinical stability not only being a time-varying confounder, but also a mediator and collider in the association between de-escalation (E) and mortality (O). Firstly, it is a confounder because it is a cause of future exposure (E) (the decision to de-escalate or not) and is also a prognostic factor for the outcome $(\mathrm{O})$ mortality. For example in the figure $C_{1}$ influences $E_{1}$ and $O$, either directly or through $C_{t}$. Secondly, clinical stability at a certain day $\left(C_{t}\right)$ is influenced by past exposure $\left(E_{1}\right.$; de-escalation or continuation of empirical therapy) and is a risk factor for mortality (O); it is therefore an intermediate between $\mathrm{E} 1$ and $\mathrm{O}$. At the same time, $\mathrm{C}_{\mathrm{t}}$ is also influenced by clinical stability from the previous day $\left(C_{1}\right)$ and is, therefore, also a collider.

The tackle these complicated relationships we provide methodological recommendations for future studies for how to deal with this and other important limitations in the next section.

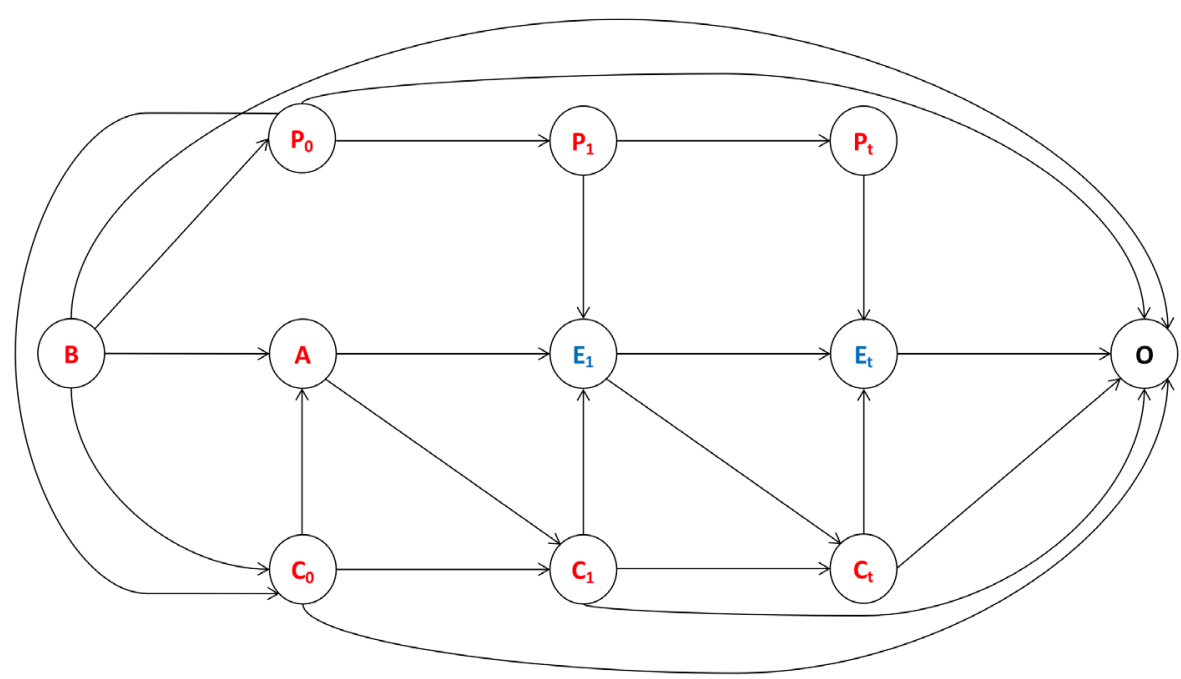

Figure 5 a directed acyclic graph (DAG) for the causal relationship between de-escalation of empirical antimicrobial therapy and mortality. Exposure $(E)=$ change or continuing antimicrobial therapy. Outcome $(\mathrm{O})=$ mortality. Time-fixed confounder $(A)=$ empirical therapy. Other time-fixed confounders $(B)=$ e.g. comorbidities and disease severity. Time-varying confounders $(P)=$ pathogen detection and $(C)=$ clinical stability. 0 and 1 are day 0 and day 1 , all other admission days are presented as $t$. 


\section{METHODOLOGICAL RECOMMENDATIONS FOR OBSERVATIONAL (DE-ESCALATION) STUDIES}

For future observational studies we provide the following recommendations.

First, the exposure variable (de-escalation) has to be included as a time-varying variable to prevent immortal time bias (also called time-dependent bias). The difference between time-fixed and time-varying exposures has been nicely explained by Munoz-Price et al. Antibiotic exposure is often available in patients' medical records and can, therefore, be determined on a daily basis. Cox proportional hazard models can be used to adjust for time-varying exposures ${ }^{[70]}$.

Second, in our opinion, important fixed confounders to consider in the analyses are patient demographics, disease severity score, comorbidities and (appropriate) empirical antimicrobial therapy, all measured at baseline. These are based on our summary in Table 2 and can all be considered as common causes of exposure and outcome. Also, source control (which was never included in the observational studies), should be considered. Still, it is the responsibility of the researcher to choose the appropriate confounders. Some of these will be specific for different infectious diseases. E.g. source control can be relevant for abdominal infections and pneumonia but not for urinary tract infections. It is beyond the scope of this paper to elaborate further on this topic. Drawing a causal diagram can be extremely helpful to determine which variables are to be considered a confounder, however a limitation of DAGs is that they cannot easily visualize interactions. Which can be a relevant part of causal inference.

Thirdly, it is inappropriate to exclusively include confounders measured at the time of admission. Confounders that change over time, are associated with the outcome, and influence the decision for de-escalation, such as clinical stability, should also be included. Clinical stability, as a concept, is not easily measured. To the best of our knowledge, there are criteria defined by the Infectious Diseases Society of America (IDSA) for non-ICU hospitalized patients with CAP but not for other populations ${ }^{[73]}$. Clinical stability in CAP patients is reached when temperature $\leq 37.8^{\circ} \mathrm{C}$, heart rate $\leq 100$ beats $/ \mathrm{min}$, respiratory rate $\leq 24$ breaths/min, systolic blood pressure $\geq 90 \mathrm{~mm} \mathrm{Hg}$, arterial oxygen saturation $\geq 90 \%$ or pO2 $\geq 60 \mathrm{~mm} \mathrm{Hg}$ on room air. For the switch to oral treatment, two variables are added: ability to maintain oral intake and normalized mental status. For disease entities where such criteria are not established, researchers should determine criteria for clinical stability. The availability of accepted criteria does not exclude the researchers responsibility to critically consider which other factors (e.g. oral food intake) determine the decision for de- 
escalation, as these can vary locally. Clinical stability should be modeled as a time-varying confounder because individual patients become clinical stable at different time points.

A complicating factor is that clinical stability is influenced by past exposure; de-escalation or continuation of empirical antimicrobial therapy may influence clinical stability of a patient during the subsequent hospital stay, for example by clinical deterioration after deescalation. As a result, conventional Cox proportional hazard models may provide biased estimates caused by adjustment for an intermediate and a collider (collider-stratification bias), as explained in the previous section ${ }^{[74,75]}$. For time-varying confounders that are influenced by past exposure G-methods are proposed ${ }^{[76]}$. The G-methods comprise three statistical causal methods: G-computation algorithm formula, G estimation of a structural nested model, and inverse-probability-of-treatment weighted (IPTW) marginal structural models (MSM) ${ }^{[76]}$. Recently, statistical software has also become available for other methods, such as the 'gfoRmula' and 'DTReg' package in R. For IPTW MSM statistical software is available ${ }^{[78]}$ and the model will provide correct estimates for associations between de-escalation of empirical antimicrobial therapy and mortality, provided that the model assumptions are met. MSM was first introduced by Robins and Hernán ${ }^{[75]}$, interested readers may also consider the more accessible introductions to these models ${ }^{[77-79]}$, or the more technical tutorial of Daniel et al ${ }^{[80]}$. Space prohibits an elaborate discussion on these model assumptions.

Ideally, clinical stability is determined daily until patients are not at risk for the exposure (de-escalation) anymore, which is after the last day of antibiotic therapy (or after hospital discharge or death). A disadvantage of current MSM implementations in statistical software is that exposure can occur only once, and the model cannot incorporate escalation after prior de-escalation (it could be done, but you have to do this by hand, requiring statistical expertise). Alternatively, researchers might simply report the crude proportion of escalations occurring after de-escalation. Unfortunately, in real-life situations missing data in the daily measurements of clinical stability may occur, for which in most circumstances multiple imputation is the recommended approach.

Obviously, the better alternative to avoid confounding by indication is a trial in which randomization for de-escalation or continuation is performed when patients are clinically stable and/or culture results are known. However, clinical trials are time consuming and expensive, not all indications can be studied in a trial, and not all at the same time, making observational studies, when performed correctly and with the right data, valuable alternatives until trial data become available. When planning a randomized trial, such observational studies can be useful to generate hypotheses and inform the design of the trial. 


\section{CONCLUSIONS}

The current evidence base on the safety of de-escalation of empirical antimicrobial therapy contains one RCT and 52 observational studies that suffer from various methodological limitations. Future observational studies could be improved by using advanced statistical analyses such as IPTW MSM to adjust for the time-varying exposure of de-escalation and the time-varying confounding effect of clinical stability during hospital stay.

\section{FIVE-YEAR VIEW}

In the next 5 years researchers and clinicians should establish a standardized definition for de-escalation and clinical stability for specific infections, particularly outside the ICU, which should be developed as a continuous score rather than a binary variable. Also, an important goal is to determine the causal effect of de-escalation on mortality either by well-designed RCTs or by observational studies using appropriate methodology, such as MSM.

\section{KEY ISSUES}

- De-escalation as an antimicrobial stewardship strategy is mainly based on observational data.

- The protective effect on mortality for de-escalation in observational studies is likely due to confounding by indication.

- De-escalation in observational studies should be analysed with techniques that take time-varying variables into account.

\section{List of abbreviations}

APACHE II, Acute Physiology and Chronic Health Evaluation; CPIS, Clinical pulmonary infection score; DAG, directed acyclic graphs; ICU, intensive care unit; ISS, Injury Severity Score; PBS, Pitt bacteremia score; RCT, randomized controlled trial; SOFA, sequential organ failure assessment.

\section{Acknowledgements}

None. 


\section{Funding}

This research received no specific grant from any funding agency in the public, commercial, or not-for-profit sectors.

\section{Conflicts of interests}

The authors report no conflicts of interest. 


\section{REFERENCES}

1. Dellit TH, Owens RC, McGowan JE, Jr. et al. Infectious Diseases Society of America and the Society for Healthcare Epidemiology of America guidelines for developing an institutional program to enhance antimicrobial stewardship. Clin Infect Dis, 44(2), 159-177 (2007).

2. Schuts EC, Hulscher MEJL, Mouton JW et al. SWAB guidelines for Antimicrobial Stewardship. (Ed.^(Eds) (SWAB, 2016) 847-856.

3. Leone $\mathrm{M}$, Bechis $\mathrm{C}$, Baumstarck $\mathrm{K}$ et al. De-escalation versus continuation of empirical antimicrobial treatment in severe sepsis: a multicenter non-blinded randomized noninferiority trial. Intensive Care Med. 40(10), 1399-1408 (2014).

* Multi-center trial evaluating the effects of de-escalation.

4. Kim JW, Chung J, Choi SH et al. Early use of imipenem/cilastatin and vancomycin followed by de-escalation versus conventional antimicrobials without de-escalation for patients with hospital-acquired pneumonia in a medical ICU: a randomized clinical trial. Crit Care, 16(1), R28 (2012).

5. Moriyama Y, Ishikane M, Mezaki K, Ohmagari N. Comparison of penicillins (penicillin G and ampicillin) and cefazolin as a definitive therapy against penicillin-susceptible Staphylococcus aureus (PSSA) bacteremia in Japan: a retrospective cohort study. Journal of infection and chemotherapy : official journal of the Japan Society of Chemotherapy, 26(4), 358-362 (2020).

6. Ambaras Khan R, Aziz Z. Antibiotic de-escalation in patients with pneumonia in the intensive care unit: A systematic review and meta-analysis. Int J Clin Pract, 72(10), e13245 (2018).

7. Guo Y, Gao W, Yang H, Ma C, Sui S. De-escalation of empiric antibiotics in patients with severe sepsis or septic shock: A meta-analysis. Heart Lung, 45(5), 454-459 (2016).

8. Ohji G, Doi A, Yamamoto S, Iwata K. Is de-escalation of antimicrobials effective? A systematic review and meta-analysis. Int J Infect Dis, 49, 71-79 (2016).

9. Paul M, Dickstein $Y$, Raz-Pasteur A. Antibiotic de-escalation for bloodstream infections and pneumonia: systematic review and meta-analysis. Clin Microbiol Infect, 22(12), 960-967 (2016).

10. Tabah A, Cotta MO, Garnacho-Montero J et al. A Systematic Review of the Definitions, Determinants, and Clinical Outcomes of Antimicrobial De-escalation in the Intensive Care Unit. Clin Infect Dis, 62(8), 1009-1017 (2016).

11. Elhanan G, Sarhat M, Raz R. Empiric antibiotic treatment and the misuse of culture results and antibiotic sensitivities in patients with community-acquired bacteraemia due to urinary tract infection. J Infect, 35(3), 283-288 (1997).

12. Alvarez-Lerma F, Alvarez B, Luque $\mathrm{P}$ et al. Empiric broad-spectrum antibiotic therapy of nosocomial pneumonia in the intensive care unit: a prospective observational study. Crit Care, 10(3), R78 (2006).

13. Berild D, Mohseni A, Diep LM, Jensenius M, Ringertz SH. Adjustment of antibiotic treatment according to the results of blood cultures leads to decreased antibiotic use and costs. J Antimicrob Chemother, 57(2), 326330 (2006).

14. Kollef MH, Morrow LE, Niederman MS et al. Clinical characteristics and treatment patterns among patients 
with ventilator-associated pneumonia. Chest, 129(5), 1210-1218 (2006).

15. Giantsou E, Liratzopoulos N, Efraimidou E et al. De-escalation therapy rates are significantly higher by bronchoalveolar lavage than by tracheal aspirate. Intensive Care Med, 33(9), 1533-1540 (2007).

16. Joffe AR, Muscedere J, Marshall JC, Su Y, Heyland DK, Canadian Critical Care Trials G. The safety of targeted antibiotic therapy for ventilator-associated pneumonia: a multicenter observational study. J Crit Care, 23(1), 82-90 (2008).

17. Eachempati SR, Hydo LJ, Shou J, Barie PS. Does de-escalation of antibiotic therapy for ventilator-associated pneumonia affect the likelihood of recurrent pneumonia or mortality in critically ill surgical patients? $J$ Trauma, 66(5), 1343-1348 (2009).

18. De Waele JJ, Ravyts M, Depuydt P, Blot SI, Decruyenaere J, Vogelaers D. De-escalation after empirical meropenem treatment in the intensive care unit: fiction or reality? J Crit Care, 25(4), 641-646 (2010).

19. Morel J, Casoetto J, Jospe R et al. De-escalation as part of a global strategy of empiric antibiotherapy management. A retrospective study in a medico-surgical intensive care unit. Crit Care, 14(6), R225 (2010).

20. Schlueter M, James C, Dominguez A, Tsu L, Seymann G. Practice patterns for antibiotic de-escalation in culture-negative healthcare-associated pneumonia. Infection, 38(5), 357-362 (2010).

21. Joung MK, Lee JA, Moon SY et al. Impact of de-escalation therapy on clinical outcomes for intensive care unit-acquired pneumonia. Crit Care, 15(2), R79 (2011).

22. Schweizer ML, Furuno JP, Harris AD et al. Comparative effectiveness of nafcillin or cefazolin versus vancomycin in methicillin-susceptible Staphylococcus aureus bacteremia. BMC Infect Dis, 11, 279 (2011).

23. Shime N, Satake S, Fujita N. De-escalation of antimicrobials in the treatment of bacteraemia due to antibioticsensitive pathogens in immunocompetent patients. Infection, 39(4), 319-325 (2011).

24. Heenen S, Jacobs F, Vincent JL. Antibiotic strategies in severe nosocomial sepsis: why do we not de-escalate more often? Crit Care Med, 40(5), 1404-1409 (2012).

25. Gonzalez L, Cravoisy A, Barraud D et al. Factors influencing the implementation of antibiotic de-escalation and impact of this strategy in critically ill patients. Crit Care, 17(4), R140 (2013).

26. Knaak E, Cavalieri SJ, Elsasser GN, Preheim LC, Gonitzke A, Destache CJ. Does Antibiotic De-escalation for Nosocomial Pneumonia Impact Intensive Care Unit Length of Stay? Infectious Diseases in Clinical Practice, 21(3), 172-176 (2013).

27. Shime N, Kosaka T, Fujita N. De-escalation of antimicrobial therapy for bacteraemia due to difficult-to-treat Gram-negative bacilli. Infection, 41(1), 203-210 (2013).

28. Cremers AJ, Sprong T, Schouten JA et al. Effect of antibiotic streamlining on patient outcome in pneumococcal bacteraemia. J Antimicrob Chemother, 69(8), 2258-2264 (2014).

29. Garnacho-Montero J, Gutierrez-Pizarraya A, Escoresca-Ortega A et al. De-escalation of empirical therapy is associated with lower mortality in patients with severe sepsis and septic shock. Intensive Care Med, 40(1), 32-40 (2014).

30. Khasawneh FA, Karim A, Mahmood T, Ahmed S, Jaffri SF, Mehmood M. Safety and feasibility of antibiotic deescalation in bacteremic pneumonia. Infect Drug Resist, 7, 177-182 (2014).

31. Khasawneh FA, Karim A, Mahmood T et al. Antibiotic de-escalation in bacteremic urinary tract infections: 
potential opportunities and effect on outcome. Infection, 42(5), 829-834 (2014).

32. Koupetori M, Retsas T, Antonakos $\mathrm{N}$ et al. Bloodstream infections and sepsis in Greece: over-time change of epidemiology and impact of de-escalation on final outcome. BMC Infect Dis, 14, 272 (2014).

33. Mokart D, Slehofer G, Lambert J et al. De-escalation of antimicrobial treatment in neutropenic patients with severe sepsis: results from an observational study. Intensive Care Med, 40(1), 41-49 (2014).

34. Carugati M, Franzetti F, Wiemken Tet al. De-escalation therapy among bacteraemic patients with communityacquired pneumonia. Clin Microbiol Infect, 21(10), 936 e911-938 (2015).

35. Lee CC, Lee NY, Chen PL et al. Impact of antimicrobial strategies on clinical outcomes of adults with septic shock and community-onset Enterobacteriaceae bacteremia: de-escalation is beneficial. Diagn Microbiol Infect Dis, 82(2), 158-164 (2015).

36. Lew KY, Ng TM, Tan M et al. Safety and clinical outcomes of carbapenem de-escalation as part of an antimicrobial stewardship programme in an ESBL-endemic setting. J Antimicrob Chemother, 70(4), 1219-1225 (2015)

37. Loo LW, Liew YX, Lee W, Chlebicki P, Kwa AL. Impact of Antimicrobial Stewardship Program (ASP) on Outcomes in Patients with Acute Bacterial Skin and Skin Structure Infections (ABSSSIs) in an Acute-Tertiary Care Hospital. Infect Dis Ther, 4(Suppl 1), 15-25 (2015).

38. De Bus L, Denys W, Catteeuw J et al. Impact of de-escalation of beta-lactam antibiotics on the emergence of antibiotic resistance in ICU patients: a retrospective observational study. Intensive Care Med, 42(6), 10291039 (2016)

39. Johnson LS, Patel D, King EA, Maslow JN. Impact of microbiology cascade reporting on antibiotic deescalation in cefazolin-susceptible Gram-negative bacteremia. Eur J Clin Microbiol Infect Dis, 35(7), 1151-1157 (2016).

40. Liu P, Ohl C, Johnson J, Williamson J, Beardsley J, Luther V. Frequency of empiric antibiotic de-escalation in an acute care hospital with an established Antimicrobial Stewardship Program. BMC Infect Dis, 16(1), 751 (2016).

41. Montravers P, Augustin P, Grall N et al. Characteristics and outcomes of anti-infective de-escalation during health care-associated intra-abdominal infections. Crit Care, 20, 83 (2016).

42. Moraes RB, Guillen JA, Zabaleta WJ, Borges FK. De-escalation, adequacy of antibiotic therapy and culture positivity in septic patients: an observational study. Rev Bras Ter Intensiva, 28(3), 315-322 (2016).

43. Oshima T, Kodama Y, Takahashi W et al. Empiric Antibiotic Therapy for Severe Sepsis and Septic Shock. Surg Infect (Larchmt), 17(2), 210-216 (2016)

44. Turza KC, Politano AD, Rosenberger LH, Riccio LM, McLeod M, Sawyer RG. De-Escalation of Antibiotics Does Not Increase Mortality in Critically III Surgical Patients. Surg Infect (Larchmt), 17(1), 48-52 (2016).

45. West DM, McCauley LM, Sorensen JS, Jephson AR, Dean NC. Pneumococcal urinary antigen test use in diagnosis and treatment of pneumonia in seven Utah hospitals. ERJ Open Res, 2(4) (2016).

46. Yamana H, Matsui H, Tagami T, Hirashima J, Fushimi K, Yasunaga H. De-escalation versus continuation of empirical antimicrobial therapy in community-acquired pneumonia. J Infect, 73(4), 314-325 (2016).

47. Blot M, Pivot D, Bourredjem A et al. Effectiveness of and obstacles to antibiotic streamlining to amoxicillin 
monotherapy in bacteremic pneumococcal pneumonia. Int J Antimicrob Agents, 50(3), 359-364 (2017).

48. Deconinck L, Meybeck A, Patoz P et al. Impact of combination therapy and early de-escalation on outcome of ventilator-associated pneumonia caused by Pseudomonas aeruginosa. Infect Dis (Lond), 49(5), 396-404 (2017).

49. Fu R, Chen T, Song J et al. De-escalation empirical antibiotic therapy improved survival for patients with severe aplastic anemia treated with antithymocyte globulin. Medicine (Baltimore), 96(6), e5905 (2017).

50. Khan RA, Aziz Z. A retrospective study of antibiotic de-escalation in patients with ventilator-associated pneumonia in Malaysia. Int J Clin Pharm, 39(4), 906-912 (2017).

51. Lee CC, Wang JL, Lee $\mathrm{CH}$ et al. Clinical benefits of antimicrobial de-escalation in adults with communityonset monomicrobial Escherichia coli, Klebsiella species and Proteus mirabilis bacteremia. Int J Antimicrob Agents, 50(3), 371-376 (2017).

52. Ni Riain U, Tierney M, Doyle C, Vellinga A, Fleming C, Cormican M. Targeted de-escalation rounds may effectively and safely reduce meropenem use. Ir J Med Sci, 186(3), 729-732 (2017).

53. Trupka T, Fisher K, Micek ST, Juang P, Kollef MH. Enhanced antimicrobial de-escalation for pneumonia in mechanically ventilated patients: a cross-over study. Crit Care, 21(1), 180 (2017).

54. Viasus D, Simonetti AF, Garcia-Vidal C, Niubo J, Dorca J, Carratala J. Impact of antibiotic de-escalation on clinical outcomes in community-acquired pneumococcal pneumonia. J Antimicrob Chemother, 72(2), 547$553(2017)$

55. Xiao B, Wang M, Hu X, Li J, Wang F, Sun J. Antibiotic de-escalation principle in elderly patients with chronic obstructive pulmonary disease complicated with severe pneumonia. Exp Ther Med, 13(4), 1485-1489 (2017).

56. Gustinetti G, Raiola AM, Varaldo R et al. De-Escalation and Discontinuation of Empirical Antibiotic Treatment in a Cohort of Allogeneic Hematopoietic Stem Cell Transplantation Recipients during the Pre-Engraftment Period. Biol Blood Marrow Transplant, 24(8), 1721-1726 (2018).

57. Li H, Yang CH, Huang LO et al. Antibiotics De-Escalation in the Treatment of Ventilator-Associated Pneumonia in Trauma Patients: A Retrospective Study on Propensity Score Matching Method. Chin Med J (Engl), 131(10), 1151-1157 (2018)

58. Palacios-Baena ZR, Delgado-Valverde M, Valiente Mendez A et al. Impact of de-escalation on prognosis of patients with bacteraemia due to Enterobacteriaceae: a post-hoc analysis from a multicenter prospective cohort. Clin Infect Dis, (2018)

59. You AS, Fukunaga BT, Hanlon AL, Lozano AJ, Goo RA. The Daniel K. Inouye College of Pharmacy Scripts: The Effects of Vancomycin Use and De-escalation in Patients Hospitalized with Pneumonia. Hawaii J Med Public Health, 77(10), 261-267 (2018).

60. Cowley MC, Ritchie DJ, Hampton N, Kollef MH, Micek ST. Outcomes Associated With De-escalating Therapy for Methicillin-Resistant Staphylococcus aureus in Culture-Negative Nosocomial Pneumonia. Chest, 155(1), 53-59 (2019)

61. Sadyrbaeva-Dolgova S, Aznarte-Padial P, Pasquau-Liano J, Exposito-Ruiz M, Calleja Hernandez MA, HidalgoTenorio C. Clinical outcomes of carbapenem de-escalation regardless of microbiological results: A propensity score analysis. Int J Infect Dis, 85, 80-87 (2019). 
62. van Heijl I, Schweitzer VA, Boel CHE et al. Confounding by indication of the safety of de-escalation in community-acquired pneumonia: A simulation study embedded in a prospective cohort. PLoS One, 14(9), e0218062 (2019).

63. Knaus WA, Draper EA, Wagner DP, Zimmerman JE. APACHE II: a severity of disease classification system. Crit Care Med, 13(10), 818-829 (1985).

64. Schurink CAM, Nieuwenhoven CAV, Jacobs JA et al. Clinical pulmonary infection score for ventilatorassociated pneumonia: accuracy and inter-observer variability. Intensive Care Med, 30(2), $217-224$ (2004).

65. Vincent JL, Moreno R, Takala J et al. The SOFA (Sepsis-related Organ Failure Assessment) score to describe organ dysfunction/failure. On behalf of the Working Group on Sepsis-Related Problems of the European Society of Intensive Care Medicine. Intensive Care Med, 22(7), 707-710 (1996).

66. Henderson H, Luterbach CL, Cober E et al. The Pitt Bacteremia Score Predicts Mortality in Non-Bacteremic Infections. Clin Infect Dis, (2019)

67. Halm EA, Fine MJ, Marrie TJ et al. Time to clinical stability in patients hospitalized with community-acquired pneumonia: implications for practice guidelines. Jama, 279(18), 1452-1457 (1998).

68. Castro-Guardiola A, Viejo-Rodriguez AL, Soler-Simon S et al. Efficacy and safety of oral and early-switch therapy for community-acquired pneumonia: a randomized controlled trial. Am J Med, 111(5), 367-374 (2001).

69. Oosterheert JJ, Bonten MJ, Schneider MM et al. Effectiveness of early switch from intravenous to oral antibiotics in severe community acquired pneumonia: multicentre randomised trial. BMJ (Clinical research ed.), 333(7580), 1193 (2006).

70. Munoz-Price LS, Frencken JF, Tarima S, Bonten M. Handling Time-dependent Variables: Antibiotics and Antibiotic Resistance. Clin Infect Dis, 62(12), 1558-1563 (2016)

71. Hernan MA, Hernandez-Diaz S, Werler MM, Mitchell AA. Causal knowledge as a prerequisite for confounding evaluation: an application to birth defects epidemiology. Am J Epidemiol, 155(2), 176-184 (2002).

72. Pearl J. Causality (Cambridge University Press, Cambridge, United Kingdom, 2000).

73. Mandell LA, Wunderink RG, Anzueto A et al. Infectious Diseases Society of America/American Thoracic Society consensus guidelines on the management of community-acquired pneumonia in adults. Clinical infectious diseases : an official publication of the Infectious Diseases Society of America, 44 Suppl 2, S27-72 (2007).

74. Hernan MA, Brumback B, Robins JM. Marginal structural models to estimate the causal effect of zidovudine on the survival of HIV-positive men. Epidemiology (Cambridge, Mass.), 11(5), 561-570 (2000).

75. Robins JM, Hernan MA, Brumback B. Marginal structural models and causal inference in epidemiology. Epidemiology (Cambridge, Mass.), 11(5), 550-560 (2000).

76. Robins J, Hernan M. Estimation of the causal effects of time-varying exposures. In: Handbooks of Modern Statistical Methods: Advances Longitudinal Data Analysis. Fitzmaurice, G, Davidian, M, Verbeke, G, Molenberghs, G (Eds.) (Chapman \& Hall/CRC (Taylor \& Francis Group), Boca Raton, FL, 2009) 553-599.

77. Naimi Al, Cole SR, Kennedy EH. An introduction to g methods. International journal of epidemiology, 46(2), 756-762 (2017)

* A simple example illustrating all G methods, with minimal technical complications.

78. Mansournia MA, Etminan M, Danaei G, Kaufman JS, Collins G. Handling time varying confounding in 
observational research. BMJ (Clinical research ed.), 359, j4587 (2017).

* A very accesible article on handling time-varying confounding.

79. Williamson T, Ravani P. Marginal structural models in clinical research: when and how to use them? Nephrol Dial Transplant, 32(suppl_2), ii84-ii90 (2017).

* * A very accesible article on MSM.

80. Daniel RM, Cousens SN, De Stavola BL, Kenward MG, Sterne JA. Methods for dealing with time-dependent confounding. Statistics in medicine, 32(9), 1584-1618 (2013). 


\section{Appendix 1 Search strategy}

(("Drug Substitution"[Mesh] OR pathogen directed [tw] OR small spectrum[tw] OR narrow spectrum[tw] OR (tailored[tw] AND (therapy[tw] OR therapies[tw] OR treatment*[tw])) OR ((broad[tw] OR empiric*[tw]) AND (narrow[tw] OR change*[tw] OR switch*[tw] OR substitut*[tw])) OR streamlin*[tw] or de-escalat*[tw]) AND ((("Anti-Bacterial Agents" [Mesh] OR "Anti-Bacterial Agents" [Pharmacological Action] OR "Antifungal Agents"[Mesh] OR "Antifungal Agents" [Pharmacological Action] OR antibacterial*[tw] OR antibacterial*[tw] OR anti-mycobacterial*[tw] OR antimycobacterial*[tw] OR antibiotic*[tiab] OR anti-infective[tiab] OR antifungal[tiab] OR anti-fungal[tiab] OR bactericid*[tw] OR bacteriocid*[tw]) AND ("Outcome Assessment (Health Care)"[Mesh] OR outcome*[tw] OR "DrugRelated Side Effects and Adverse Reactions"[Mesh] OR "adverse effects" [Subheading] OR adverse effect*[tw] OR adverse reaction*[tw] OR adverse drug reaction*[tw] OR adverse event*[tw] OR adverse drug event*[tw] OR undesirable effect*[tw] OR side effect*[tw] OR "Mortality"[Mesh] OR mortalit*[tw] OR "mortality"[Subheading] OR death*[tw] OR fatal*[tw] OR "Morbidity"[Mesh:NoExp] OR morbidit*[tw] OR "Drug Resistance"[Mesh] OR resistan*[tw] OR "Costs and Cost Analysis"[Mesh] OR cost*[tw])) NOT case reports[pt]) NOT ("HIV Infections"[Mesh] OR HIV[Mesh] OR HIV[ti] OR human immunodeficiency virus[ti])) AND 2014/04:2019/02 [edat])) 
abo

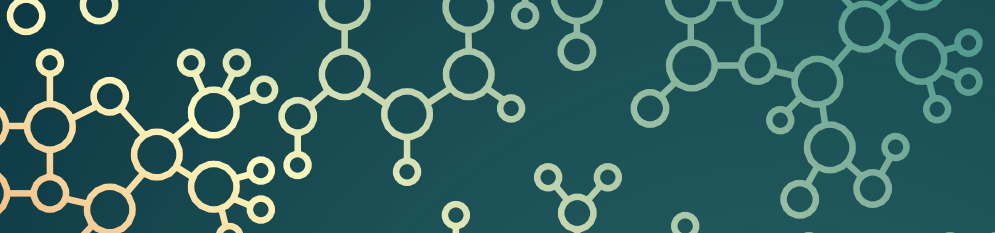
o

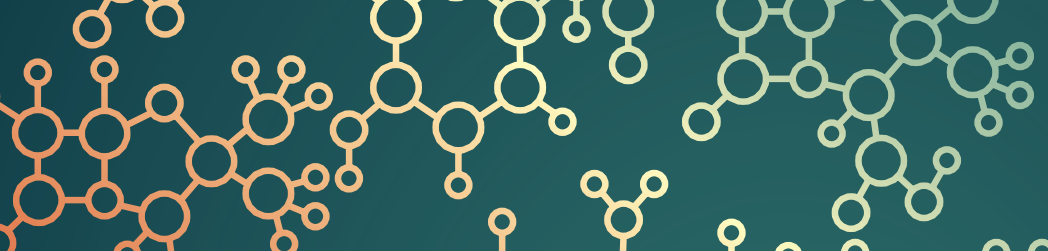

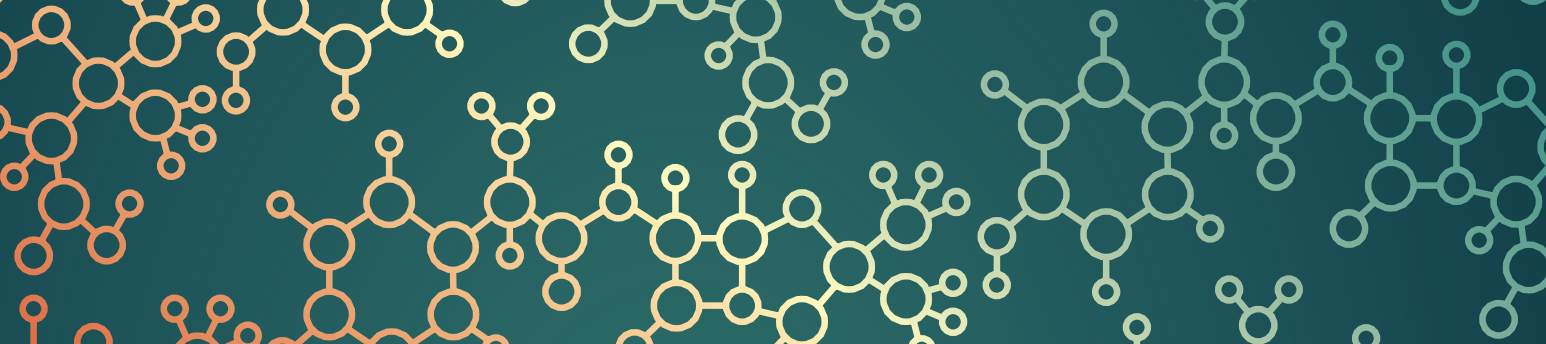

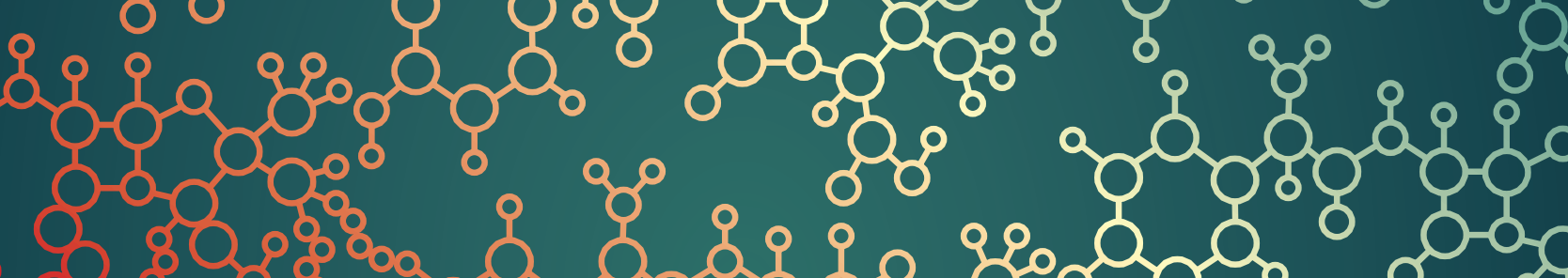
80\%

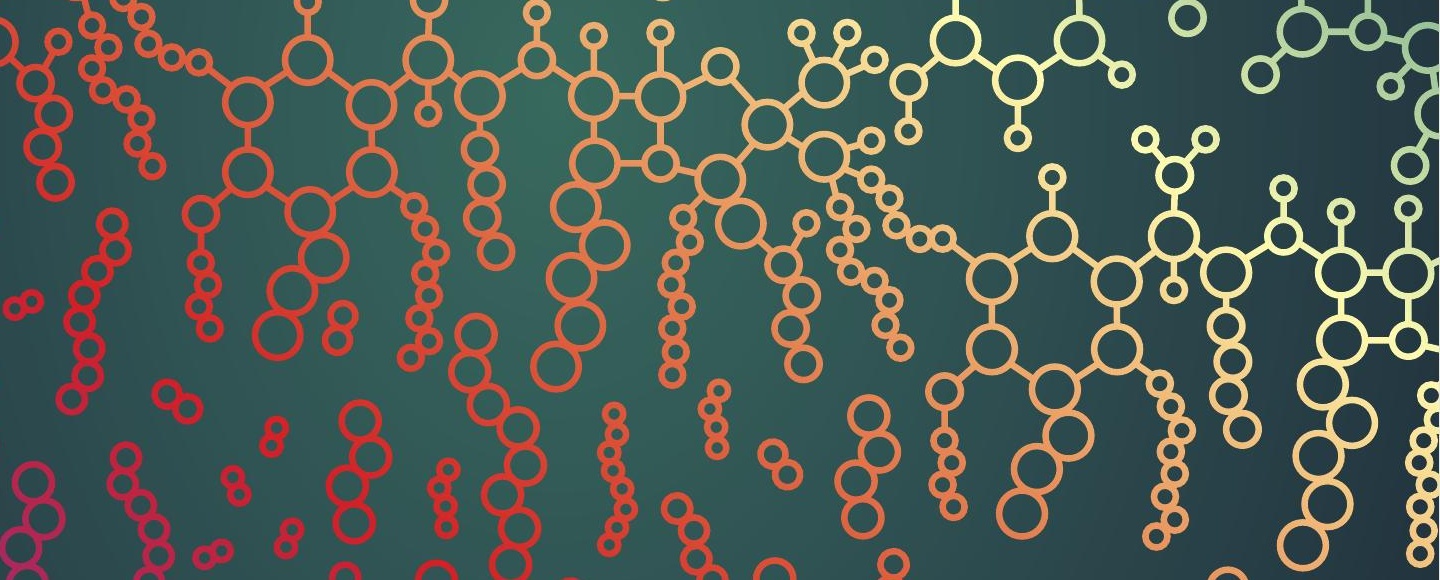
$88 \% 888$ $\circ 88$ \& 8

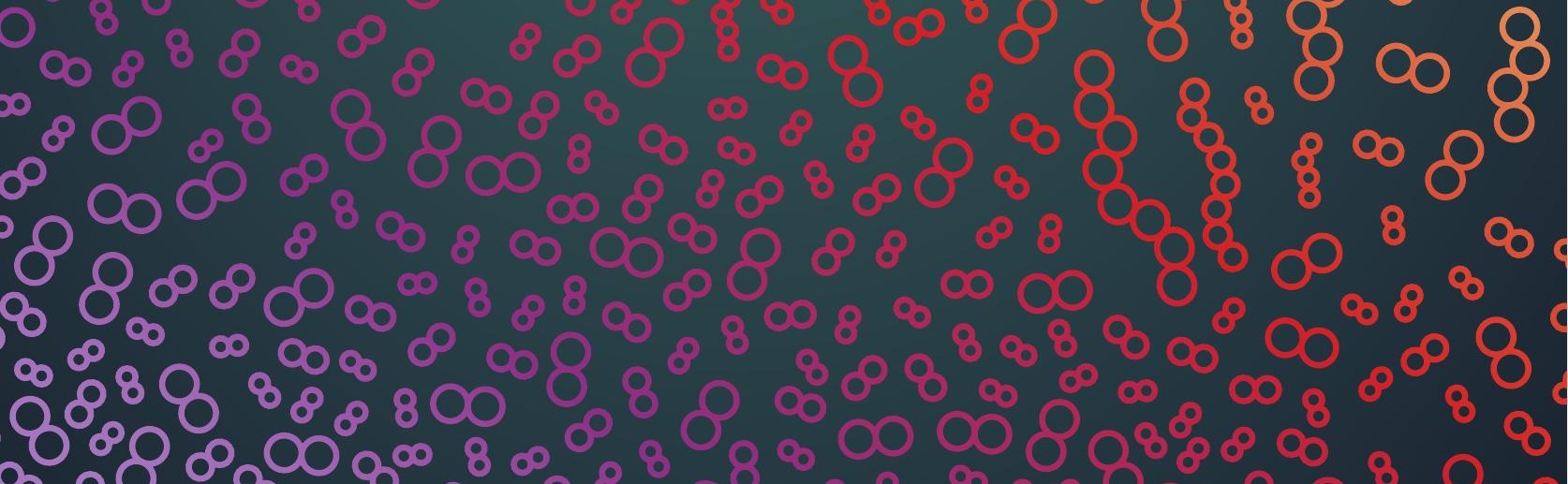


Summary and general discussion 


\section{SUMMARY AND GENERAL DISCUSSION}

This thesis aimed to provide evidence that can be used to improve treatment of hospitalized patients with community-acquired pneumonia (CAP) (part I) and provides recommendations to improve the methodology of the evidence on which treatment recommendations are based (part II). Part I provides evidence and recommendations for appropriate antibiotic use in elderly patients and presents the results of a multicentre study with an antibiotic stewardship intervention, implemented in twelve Dutch hospitals, that safely reduced unnecessary broad-spectrum antibiotic use. Part II provides several evaluations of the methodologies used in published ASP studies, (in particular in studies evaluating de-escalation of empirical therapy) with recommendations for designing future studies. Each part will end with future perspectives on research and clinical practice.

\section{Part I: Antibiotic stewardship strategies in CAP}

In acute care hospitals 20-50\% of the prescriptions for antibiotic therapy are considered inappropriate ${ }^{[1-5]}$. Inappropriate use of antibiotics contributes to selection of antibiotic resistance, increases healthcare costs and risk of complications ${ }^{[6]}$. Additionally, prescribing appropriate antibiotic therapy specifically in elderly (adults over 64 years of age) patients seems quite a challenge, possibly because they often present with atypical symptoms. Moreover elderly patients often reside in long-term care facilities (LTCF) or nursing homes, which are risk factors for developing infections with antibiotic resistant bacteria. Elderly patients are more prone to develop adverse events (ADE) and drugdrug interactions because of co-morbidities and/or polypharmacy ${ }^{[9]}$. In Chapter 2 we review different aspects of antibiotic use in lower respiratory tract infections (and in CAP specifically) in elderly patients, which are important to consider when determining appropriateness of therapy in this population. First, the microbial aetiology is only slightly different for elderly patients compared to the younger population, and the mainstay of treatment should consist of beta-lactam monotherapy. Extended coverage of gramnegatives could be considered in LTCF or nursing home residents. Empirical treatment in combination with fluoroquinolones or macrolides should be restricted to selected cases given the low incidence of atypical pathogens in elderly patients with CAP and higher risks of ADEs and drug-drug interactions. Next to these ADEs, inappropriate use of antibiotics contributes to Clostridium difficile infections and antibiotic resistance. A well-designed antibiotic stewardship program (ASP) could improve antibiotic prescribing, but studies specifically targeting elderly patients are lacking and thus needed. These programs should generally consist of multiple components depending on the specific clinical setting, such as improving diagnostics (i.e. indication for starting antibiotics) and ensuring proper dosing and duration of therapy. 
One example of a well-designed antibiotic stewardship intervention is presented in Chapter 3 and 4 . In clinical practice, guideline adherence in patients with CAP is low ${ }^{[8]}$, and ASP may improve guideline adherence and appropriateness of empirical antibiotic therapy ${ }^{[6]}$. Therefore the "Community-Acquired Pneumonia increasing Protocol adherence by Antibiotic stewardship in a stepped-wedge Cluster-randomized Trial" (CAP-PACT) trial, an investigator-initiated stepped-wedge cluster randomized quality improvement trial was performed from November 2015 until November 2017 (ClinicalTrials.gov number, NCT02604628.) Twelve Dutch hospitals participated in this trial. The intervention consisted of education, motivating opinion leaders and audit and feedback, and are considered effective in optimizing antibiotic use ${ }^{[6,10]}$. These intervention components aimed to increase guideline adherence and thereby increase the use of narrow-spectrum beta-lactam antibiotics (penicillin and amoxicillin). Among 4,084 patients (2,235 in the control and 1,849 in the intervention period) admitted with a moderate-severe CAP at a non-Intensive Care Unit (ICU), we realized a reduction of $27 \%$ in days with broad-spectrum antibiotics without compromising patient outcome (adjusted risk difference in 90 -day mortality was $0.4 \%(90 \% \mathrm{Cl}-2.7 ; 2.4))$. The results of the CAP-PACT study provide evidence that antibiotic stewardship teams (A-teams) are effective in decreasing broad-spectrum use in patients with CAP admitted to non-ICU wards.

The exact nature of each intervention component of the CAP-PACT study is described in Chapter 4. We provided a detailed description of the process evaluation of all intervention components for hospitals, where there is a need for an ASP in CAP. The process evaluation of strategies for change includes four steps: a description of (1) the intervention as planned, (2) the intervention as delivered, (3) the actual exposure to the intervention, and (4) recommendations to improve the intervention components based on experiences of the participating physicians ${ }^{[11]}$. The performed antibiotic stewardship intervention consisted of education (clinical lessons, e-learning, pocket cards and posters), motivation of and by opinion leaders, and audit and feedback with the aim to change prescribing behaviour of the physicians' into more guideline adherent prescribing. The majority of the intervention components (clinical lessons, e-learning, pocket cards and audit and feedback) were delivered as planned, apart from some minor adjustments (e.g. higher frequency of clinical lessons, lower frequency of e-learning invitations sent by e-mail). The most important recommendations to improve the individual intervention components were to also invite Emergency Room (ER) physicians' and ask supervisors to motivate residents to attend the clinical lessons. Also, hospitals could consider to schedule e-learnings during clinical lessons to gain more response. For implementation of audit and feedback it is necessary that supervisors (or local opinion leaders) support the 
guideline. At last it was suggested to use computer algorithms for the audit (and feedback) for long-term sustainability. The intervention components not delivered as planned were posters and motivation of local opinion leaders. None of the participants could remember displaying posters at the ER and, when presenting an example, participants thought the presented message was not clear. Thus, when hospitals value the use of posters it is recommended to formulate the message clearly. The planned meetings to evaluate past performances and discuss barriers that impede adherence by local opinion leaders was not performed. These meetings were suggested as an improvement by the participants, thus it is recommended to implement such meetings. In conclusion, clinical lessons, pocket cards, motivating opinion leaders and daily audit and feedback were valued most by the participants and valued as the most effective intervention components.

\section{Future perspectives}

With the CAP-PACT study a decrease of $27 \%$ in broad-spectrum antibiotic use was achieved. However, large heterogeneity between hospitals was observed, with some hospitals realizing a 17\% reduction while others had a 39\% reduction in broad-spectrum antibiotic use. Even though the intervention components of our antibiotic stewardship bundle are evidence based, it is advised to tailor the intervention to the specific setting based on behaviour change theory ${ }^{[12]}$. In a Cochrane review on ASP interventions, one of the recommendations for future studies was to explore barriers and facilitators for implementation of ASP ${ }^{[6]}$. This was also prioritized as an important research area by the Working Group on Behavioural Approaches to Antibiotic Stewardship Programs; 'conduct robust evaluations of ASPs with built-in process evaluations and fidelity assessments' [13]. In ASP studies there is the 'what' which is the goal of the study; improve appropriate antibiotic use (e.g. increase guideline adherence, increase de-escalation of empirical antibiotic therapy), and the 'how' which are the behavioural change interventions or techniques (BCTs), e.g. educational materials, audit and feedback ${ }^{[11]}$. The BCT part underlying antimicrobial stewardship interventions are under-reported in medical literature. In the CAP-PACT trial we aimed to increase guideline adherence by using a bundle of multiple intervention components. A drawback of this approach, is that it is not possible to estimate the effect of the individual components. However, the approach reflects clinical practice, where stewardship interventions are usually implemented as bundles. In a review providing evidence on recommendations for appropriate antibiotic use and BCT, also a few limitations of ASP studies are summarized ${ }^{[11]}$. First, most studies have a study design prone to confounding by indication and the majority (80\%) were conducted at one site ${ }^{[6]}$. It was recommended that future studies should have a clusterrandomized design with sufficient sites. Which we indeed did with the CAP-PACT trial. A second limitation is that most ASP studies lack a clear description of the intervention. A 
process evaluation of antibiotic stewardship interventions is important in understanding and explaining the effect (or variety in effects between different participating hospitals). Also, evaluation of the different intervention components of an ASP can be used to compare effects between ASP studies. And a detailed process evaluation provides tools for other hospitals to replicate the intervention ${ }^{[12]}$. We describe the process evaluation of the ASP implementation in the CAP-PACT trial in detail in Chapter 4, to provide useful information for hospitals in need for an ASP in CAP. Our recommendations are aimed at optimizing the intervention components, however future research should also determine the reasons for not prescribing more narrow-spectrum antibiotics when recommended. Because these reasons (barriers) may also differ between hospitals. To identify barriers and facilitators for prescribing narrow-spectrum antibiotics a questionnaire can be used based on the checklist developed by Flottorp et al ${ }^{[14]}$. This checklist is designed to identify barriers and facilitators for improvement in healthcare professional practice. With regard to the CAP-PACT trial, our one-size-fits all approach should be tailored to the needs of the hospitals. A third limitation of most ASP studies is their lack of information regarding the costs of implementation of interventions ${ }^{[12]}$. In the CAP-PACT trial we did not collect data on costs. The material costs were presumed to be low (printing of pocket cards and posters). The costs for the man-hours to prepare clinical lessons and to perform the audit and feedback are likely to be higher. Future research is needed to provide accurate information on cost-effectiveness of the CAP-PACT intervention. Finally, ASP studies rarely provide information on sustainability of behavioural change. According to the Cochrane review on ASP interventions, for example removal of restriction of certain antibiotic-classes resulted in a reversed intervention effect [6]. For the participating hospitals in the CAP-PACT trial, the intervention is still actively being implemented in eight out of nine hospitals. In seven hospitals in the context of a follow-up study (the DiagNostic Study of Low-dose CT and multipleX PCR on Antibiotic Treatment and Outcome of Community-Acquired Pneumonia; CAP-NEXT. Clinicaltrials. gov NCT03360851), in one hospital independently and in one hospital the intervention was ceased at the end of the study period. The hospital that independently continued to perform the intervention, uses a clinical decision support system (CDSS) to gain sustainability (unpublished data). Integration of CDSSs in ASP can improve efficiency of interventions ${ }^{[15]}$. A CDSS is an algorithm that links certain patient characteristics from the electronic health care system to identify the targeted population and provides patient specific advice. In practice, this algorithm runs once daily and the system generates a list of patients presumed having a CAP and receiving non-guideline adherent antibiotics. For this list the CDSS uses information on new prescriptions of specific (combination of) broad-spectrum antibiotics. Then these patients are then evaluated with feedback to the responsible physicians. However, in practice the CDSS does not use information on 
the indication, which could result in patients on this list with a different infection other than CAP. Although the current algorithm using new prescriptions of broad-spectrum antibiotics is a interesting solution for the sustainability of the intervention, it could be improved by adding information on the indication as a selection criterion by the CDSS

\section{Role of pharmacists in antibiotic stewardship programs}

The main task of a pharmacist in general is to optimise prescribing behaviour and monitor medical prescriptions mainly by using CDSSs (in the Netherlands). In 2010, the American Society of Health-System Pharmacists (ASHP) stated that pharmacists have the responsibility to play an essential role in antibiotic stewardship ${ }^{[17]}$. One of the key areas they provide concrete advices in, is promoting appropriate use of antibiotics. Examples of such advices are: ensure the availability of antibiotics, providing antibiotic usage reports for surveillance, providing information technology to enhance ASP, facilitate safe medication management, develop local antibiotic guidelines and participate in multidisciplinary infection meetings. According to a national survey on the current activities of ASP teams, performed in 2016 in 64 of 80 Dutch hospitals, the majority of the aforementioned tasks were performed as routine care, by either hospital pharmacists or by another member of the hospital antibiotic stewardship team (A-team) ${ }^{[18]}$. Monitoring and providing advices on appropriateness of antibiotic use was mainly done for restricted antibiotics (91\%), for intravenous to oral switches (53\%) and for individualized dosing with therapeutic drug monitoring (TDM) (65\%). However discontinuation of antibiotics (35\%) was far less monitored, and this is an ASP objective where pharmacists can play an important role. Based on USA data, which is probably generalizable to other Western countries, in $55-70 \%$ of patients that receive antibiotics during hospitalization, the duration of a course is inappropriately long and is often continued in the outpatient setting ${ }^{[19,20]}$. And 'each excess day of antibiotic treatment is associated with a $5 \%$ increase in the odds of antibiotic-associated adverse events reported by patients after discharge', according to a study done in patients with CAP [21]. Thus, monitoring duration, specifically at the moment patients are discharged, will improve patient outcome. In a study evaluating an intervention by pharmacy audit and feedback of discharge antibiotic prescriptions, this resulted in shorter post-hospital treatment durations ${ }^{[22]}$. In the Netherlands, discharge prescriptions are sent to outpatient pharmacies. Thus, for a hospital pharmacist to appropriately shorten the duration of antibiotic courses, they need to (1) collaborate with outpatient pharmacies and perform prospective audits of patients with antibiotic discharge prescriptions and (2) provide feedback on the duration of treatments that were prescribed.

Another ASP objective, not included in the aforementioned survey in Dutch hospitals 
to assess ASP activities ${ }^{[18]}$ or in the Dutch ASP guideline ${ }^{[23]}$, is de-labelling of penicillin allergies. In the CAP-PACT trial 13\% (127/983) of the hospitalized patients with CAP that were initially treated with broad-spectrum antibiotics could not switch to narrowspectrum antibiotics - according to treating physicians - due to penicillin allergies (Table S2, Chapter 3). This proportion of patients in our study, with a registered penicillin allergy in their medical record, is within the range of registered penicillin allergies in the UK, USA and Australia (8-25\%) ${ }^{[24-26]}$. In one study $95 \%$ of the patients with registered penicillin allergy appeared to be mislabelled ${ }^{[25]}$, which may result in unjustified broad-spectrum antibiotic use and its negative associated effects on selection for antibiotic resistance. Reducing mislabelling of penicillin allergies was therefore adopted as an objective in the USA and Australian ASP guidelines ${ }^{[27,28]}$. In an Australian hospital an ASP team led by a pharmacist identified patients with a penicillin allergy label and receiving at least one antibiotic via prospective audit of electronic medical records and evaluated these patients during ward rounds once per week ${ }^{[29]}$. These patients were reviewed by the pharmacist in consultation with an infectious disease specialist and an allergy nurse. Of 106 patients undergoing this intervention, nearly 40\% had their penicillin allergy label removed. This intervention is another ASP objective where hospital pharmacists in the Netherlands could play an important role. Exchange of such information with primary care and outpatient pharmacies may also reduce unnecessary broad-spectrum antibiotic use in the outpatient setting. With regards to the CAP-PACT trial, if we had also implemented this de-labelling intervention we could have treated more CAP patients with narrowspectrum antibiotics. 


\section{Part II Methodology used in ASP studies}

Recognition of the threat posed by antibiotic resistance and the need to optimise antibiotic prescribing has driven an exponential increase in publications of studies evaluating antibiotic stewardship interventions over the last 20 years ${ }^{[30]}$. Because most of these studies are lacking in quality, many areas of practice rely on a weak evidence base [31]; conducting clinical studies that can - because of a low-quality design - not provide evidence for guiding practice is a waste of time and valuable resources ${ }^{[6,32]}$. Journals are beginning to formulate minimum standards for antimicrobial stewardship studies to be published [33], but there is a need for consensus on how to design, analyse and report studies evaluating interventions to improve antibiotic prescribing. In Chapter 5 the results are presented of a systematic review of antimicrobial stewardship evaluations focusing on design features most important for validity and translation into clinical practice. Any study evaluating any antimicrobial stewardship intervention, without restriction on research design or outcome, was included. The initial search yielded 12,722 articles; 676 were selected for full-text screening, of which 567 were included. As in previous reports, we also found that the majority of antimicrobial stewardship studies used non-randomised research designs, with before-after studies being the most prevalent. Overall design quality of studies evaluating antibiotic stewardship interventions was low and has not improved in the past years. Most published studies did not report on clinical and microbiological outcome data. Studies conducted in primary care and studies that reported funding, were associated with higher quality. These limitations should be considered when designing future stewardship evaluations so that a robust evidence base can be built to guide clinical practice.

In Chapter 6 and 7 we zoom into the methodology used in ASP studies improving appropriateness of antibiotic use by de-escalation of empirical antibiotic therapy. We specifically evaluate studies with a non-experimental design, which are prone to confounding by indication. First, de-escalation consists of reducing the spectrum of the initial antibiotic therapy. This is the consensus of all definitions used in literature, because there is no uniform definition for de-escalation ${ }^{[34]}$. Although the exact definition may be unclear, the concept of de-escalation is widely accepted and recommended in ASP guidelines ${ }^{[23,28,35]}$. However, the safety of de-escalation of empirical antibiotic therapy is largely based on observational data, with many studies reporting protective effects of de-escalation on mortality. Since there is no plausible biological explanation for this phenomenon, it is most probably caused by unmeasured confounding by indication. Confounding by indication is present if the indication for the intervention (in this case, de-escalation of empirical antibiotic therapy) is also a prognostic factor for the outcome (mortality). De-escalation is usually only performed when clinical stability is reached in 
the first days after starting antimicrobial therapy, and clinical stability is also a strong prognostic factor for patient outcome. In Chapter 6 we performed a sensitivity analysis to quantify the potential effect of unmeasured confounding by indication due to clinical stability in the association between de-escalation and patient outcome in patients with CAP. This analysis was done by simulating a confounder representing clinical stability during hospital admission. The simulated confounder was substantially stronger than any other baseline confounder known to be important in the association between de-escalation and mortality. Quantification of effects of de-escalation on patient outcomes without proper adjustment for clinical stability, results in strong negative bias. This study suggests the effect of de-escalation on mortality needs further well-designed prospective research to determine effect size more accurately. It is clear that the methodology used in studies evaluating the effects of de-escalation on mortality requires improvement. In Chapter 7 we depicted all potential confounders in a directed acyclic graph (DAG) to illustrate all associations between exposure (de-escalation) and outcome (mortality). Clinical stability is an important confounder in this association and should be modelled as a time-varying variable. We recommend to include de-escalation as time-varying exposure and use inverse-probability-of-treatment weighted marginal structural models to properly adjust for time-varying confounders.

\section{Future perspectives}

A sensitivity analysis, as we performed in Chapter 6, is strongly recommended for observational studies to assess how robust the association is to potential unmeasured confounding. However in practice sensitivity analyses are often not performed for various reasons; researchers think it is too complicated to perform, or to describe it, or it takes too much space in the manuscript ${ }^{[36]}$. In a response to these objections in 2017 the E-value was introduced by VanderWeele et al. ${ }^{[37]}$, to make sensitivity analysis more common in clinical practice. This E-value is defined as 'the minimum strength of association, on the risk ratio scale, that an unmeasured confounder would need to have with both the treatment and the outcome to fully explain away a specific treatmentoutcome association, conditional on the measured covariates'. In other words, 'a large E-value implies that considerable unmeasured confounding would be needed to explain away an effect estimate'. VanderWeele et al. state that if observational studies start to report the E-value, results would more accurately assess the evidence for causality. How does it work? Compared to the usual sensitivity analyses, like the one we performed, you do not need to make assumptions on several parameters for the E-value ${ }^{[38]}$. Instead of making assumptions regarding the strength of the unmeasured confounder with the outcome and with the exposure, the E-value only focusses on the magnitude of the unmeasured confounder associations needed to neutralize the observed exposure- 
outcome association. It is recommended to report E-value in the manuscript with an additional comment if an unmeasured confounder of that specific magnitude is plausible. The E-value can be calculated with the following formula: $E$-value $=(1 / H R)+\sqrt{ }((1 / H R) x$ $((1 / H R)-1))$.

The 'HR' is replaceable for 'OR' or 'RR'. The E-value represents a set of 2 parameters: association between unmeasured confounder with exposure, and association with outcome. They are both equal to each other to determine the minimum for both. In practice, one could be larger then the $\mathrm{E}$-value, and thereby the other thus smaller. There is also a R Package and an online E-value calculator available ${ }^{[39]}$.

In Chapter 6 our purpose was to quantify the magnitude of a specific unmeasured important confounder ('clinical stability') which often is not included in the association between de-escalation and mortality in the available observational studies. Thus, the sensitivity analysis we used, with assumptions based on literature, was the most suitable for our purpose. The E-value is a possibility for those observational studies demonstrating lower mortality among patients with a de-escalation of antibiotic treatment, compared to patients that continued empirical treatment. For the proof of concept we used our own adjusted $\mathrm{HR}$ of 0.39 (95\% $\mathrm{Cl}: 0.19$ - 0.79) for day-30 mortality (adjusted for known and measured confounders).

With use of the Web version of the E-value calculator ${ }^{[39]}$ we obtained an E-value of 4.57. Which means that the association between de-escalation and mortality could be explained away by an unmeasured confounder that is associated with both de-escalation and mortality by a hazard ratio of 4.57- fold each (beyond the measured confounders), and the upper limit of the confidence interval $(\mathrm{Cl})$ was 1.85. Unmeasured confounders with a weaker association could not explain away the association observed between deescalation and mortality. In contrast to the E-value of the estimate, weaker confounder associations could move the $\mathrm{Cl}$ to include a hazard ratio of 1 . An unmeasured confounder associated with de-escalation and mortality with a hazard ratio of 1.85 -fold does not seem implausible. This was also concluded in Chapter 6. Still, for our purpose the extensive sensitivity analysis was more suitable. For published studies reporting protective effects for de-escalation, calculating E-values would have been helpful to conclude whether unmeasured confounding could explain away protective effects. 


\section{CONCLUSIONS}

This thesis can be summarized in the following overall conclusions:

1. A strategy to increase prescription of more narrow-spectrum antibiotics for hospitalized patients with moderate-severe CAP by an antibiotic stewardship intervention is effective and safe. (Chapter $\mathbf{3}$ )

2. Hospitals with a need for ASP in CAP can achieve a reduction in broad-spectrum antibiotics by implementing the multi-component intervention as used in the CAPPACT study. (Chapter 4)

3. Overall design quality of studies evaluating antibiotic stewardship interventions is low and has not improved in the past years (up until 2017) (Chapter 5)

4. Quantification of effects of de-escalation on patient outcomes without proper adjustment for clinical stability results in bias. (Chapter 6) Future observational studies could be improved by including de-escalation as a time-varying exposure and clinical stability as a time-varying confounder. (Chapter 7) 


\section{REFERENCES}

1. P. Davey, C. Peden, E. Charani, C. Marwick, S. Michie, Time for action-Improving the design and reporting of behaviour change interventions for antimicrobial stewardship in hospitals: Early findings from a systematic review, Int J Antimicrob Agents 45(3) (2015) 203-12.

2. M.E. Hulscher, R.P. Grol, J.W. van der Meer, Antibiotic prescribing in hospitals: a social and behavioural scientific approach, The Lancet. Infectious diseases 10(3) (2010) 167-75.

3. V. Spoorenberg, M.E. Hulscher, R.P. Akkermans, J.M. Prins, S.E. Geerlings, Appropriate antibiotic use for patients with urinary tract infections reduces length of hospital stay, Clin Infect Dis 58(2) (2014) 164-9.

4. P.C. van Spreuwel, H. Blok, M.F. Langelaar, B.J. Kullberg, J.W. Mouton, S. Natsch, Identifying targets for quality improvement in hospital antibiotic prescribing, Neth J Med 73(4) (2015) 161-8.

5. P. Zarb, B. Amadeo, A. Muller, N. Drapier, V. Vankerckhoven, P. Davey, H. Goossens, E.-H.C.S. Group, Identification of targets for quality improvement in antimicrobial prescribing: the web-based ESAC Point Prevalence Survey 2009, J Antimicrob Chemother 66(2) (2011) 443-9.

6. P. Davey, C.A. Marwick, C.L. Scott, E. Charani, K. McNeil, E. Brown, I.M. Gould, C.R. Ramsay, S. Michie, Interventions to improve antibiotic prescribing practices for hospital inpatients, Cochrane Database Syst Rev 2 (2017) Cd003543.

7. N. Adler, H. Weber, I. Gunadasa, A. Hughes, N. Friedman, Adherence to therapeutic guidelines for patients with community-acquired pneumonia in Australian hospitals, Clin Med Insights Circ Respir Pulm Med 8 (2014) 17-20.

8. S.M. Huijts, C.H. van Werkhoven, W.G. Boersma, J. Buijs, G. Buunk, C.J. Compaijen, L.J. van Elde, J.E. Gisolf, R. van der Kam, J.A. Kluytmans, B.A. Kuipers, J.J. Mager, B. Oppedijk, F. Palmen, J.M. Prins, B. van Reemst, M.H. Silbermann, F.H. van Tiel, E. van der Wall, T.S. van der Werf, M.J. Bonten, Guideline adherence for empirical treatment of pneumonia and patient outcome. Treating pneumonia in the Netherlands, Neth $J$ Med 71(10) (2013) 502-7.

9. N. Petrosillo, M.A. Cataldo, F. Pea, Treatment options for community-acquired pneumonia in the elderly people, Expert Rev Anti Infect Ther 13(4) (2015) 473-85.

10. T. Dellit, R. Owens, J. McGowan, D. Gerding, R. Weinstein, J. Burke, W.C. Huskins, D. Paterson, N. Fishman, C. Carpenter, P.J. Brennan, M. Billeter, T. Hooton, Infectious Diseases Society of America and the Society for Healthcare Epidemiology of America guidelines for developing an institutional program to enhance antimicrobial stewardship, Clinical infectious diseases 44(2) (2007) 159-177.

11. M. Hulscher, M. Laurant, R. Grol, Process evaluation of implementation strategies, in: R. Grol, M. Wensing, M. Eccles, D. Davis (Eds.), Improving patient care: the implementation of change in health care, Wiley Blackwell, Oxford, 2013, pp. 333-349.

12. M. Hulscher, J.M. Prins, Antibiotic stewardship: does it work in hospital practice? A review of the evidence base, Clin Microbiol Infect 23(11) (2017) 799-805.

13. M. Rzewuska, E. Charani, J.E. Clarkson, P.G. Davey, E.M. Duncan, J.J. Francis, K. Gillies, W.V. Kern, F. Lorencatto, C.A. Marwick, J. McEwen, R. Mohler, A.M. Morris, C.R. Ramsay, S. Rogers Van Katwyk, B. Skodvin, 
I. Smith, K.N. Suh, J.M. Grimshaw, P. Joint Programming Initiative on Antimicrobial Resistance Working Group on Behavioural Approaches to Antibiotic Stewardship, Prioritizing research areas for antibiotic stewardship programmes in hospitals: a behavioural perspective consensus paper, Clin Microbiol Infect 25(2) (2019) 163168.

14. S.A. Flottorp, A.D. Oxman, J. Krause, N.R. Musila, M. Wensing, M. Godycki-Cwirko, R. Baker, M.P. Eccles, A checklist for identifying determinants of practice: a systematic review and synthesis of frameworks and taxonomies of factors that prevent or enable improvements in healthcare professional practice, Implement Sci 8 (2013) 35 .

15. K.M. Kuper, K.W. Hamilton, Collaborative Antimicrobial Stewardship: Working with Information Technology, Infect Dis Clin North Am (2019).

16. W.J. Wiersinga, M.J. Bonten, W.G. Boersma, R.E. Jonkers, R.M. Aleva, B.J. Kullberg, J.A. Schouten, J.E. Degener, E.M.W. van de Garde, T.J. Verheij, A.P.E. Sachs, J.M. Prins, Management of community-acquired pneumonia in adults: 2016 guideline update from the Dutch Working Party on Antibiotic Policy (SWAB) and Dutch Association of Chest Physicians (NVALT), Neth J Med 76(1) (2018) 4-13.

17. ASHP statement on the pharmacist's role in antimicrobial stewardship and infection prevention and control, Am J Health Syst Pharm 67(7) (2010) 575-7.

18. M.C. Kallen, J. Ten Oever, J.M. Prins, B.J. Kullberg, J.A. Schouten, M. Hulscher, A survey on antimicrobial stewardship prerequisites, objectives and improvement strategies: systematic development and nationwide assessment in Dutch acute care hospitals, J Antimicrob Chemother 73(12) (2018) 3496-3504.

19. S.J. Scarpato, D.R. Timko, V.C. Cluzet, J.P. Dougherty, J.J. Nunez, N.O. Fishman, K.W. Hamilton, C.D.C.P.E. Program, An Evaluation of Antibiotic Prescribing Practices Upon Hospital Discharge, Infect Control Hosp Epidemiol 38(3) (2017) 353-355.

20. S.H. Yi, K.M. Hatfield, J. Baggs, L.A. Hicks, A. Srinivasan, S. Reddy, J.A. Jernigan, Duration of Antibiotic Use Among Adults With Uncomplicated Community-Acquired Pneumonia Requiring Hospitalization in the United States, Clin Infect Dis 66(9) (2018) 1333-1341.

21. V.M. Vaughn, S.A. Flanders, A. Snyder, A. Conlon, M.A.M. Rogers, A.N. Malani, E. McLaughlin, S. Bloemers, A. Srinivasan, J. Nagel, S. Kaatz, D. Osterholzer, R. Thyagarajan, L. Hsaiky, V. Chopra, T.N. Gandhi, Excess Antibiotic Treatment Duration and Adverse Events in Patients Hospitalized With Pneumonia: A Multihospital Cohort Study, Ann Intern Med 171(3) (2019) 153-163.

22. N. Yogo, K. Shihadeh, H. Young, S.L. Calcaterra, B.C. Knepper, W.J. Burman, P.S. Mehler, T.C. Jenkins, Intervention to Reduce Broad-Spectrum Antibiotics and Treatment Durations Prescribed at the Time of Hospital Discharge: A Novel Stewardship Approach, Infect Control Hosp Epidemiol 38(5) (2017) 534-541.

23. E.C. Schuts, M.E.J.L. Hulscher, J.W. Mouton, C.M. Verduin, J.W.T.C. Stuart, H.W.P.M. Overdiek, P.D. van der Linden, S. Natsch, C.M.P.M. Hertogh, T.F.W. Wolfs, J.A. Schouten, B.J. Kullberg, J.M. Prins, SWAB Guidelines for Antimicrobial Stewardship. <https://swab.nl/nl/antimicrobial-stewardship>, 2016).

24. J.R. Kerr, Penicillin allergy: a study of incidence as reported by patients, Br J Clin Pract 48(1) (1994) 5-7.

25. E. Macy, Penicillin and beta-lactam allergy: epidemiology and diagnosis, Curr Allergy Asthma Rep 14(11) (2014) 476. 
26. J.A. Trubiano, K.A. Cairns, J.A. Evans, A. Ding, T. Nguyen, M.J. Dooley, A.C. Cheng, The prevalence and impact of antimicrobial allergies and adverse drug reactions at an Australian tertiary centre, BMC Infect Dis 15 (2015) 572.

27. Australian Commission on Safety and Quality in Health Care, Antimicrobial Stewardship in Australian Health Care 2018 <https://www.safetyandquality.gov.au/our-work/antimicrobial-stewardship/antimicrobialstewardship-australian-health-care-2018>, 2018 (accessed February 1.2020).

28. T.F. Barlam, S.E. Cosgrove, L.M. Abbo, C. MacDougall, A.N. Schuetz, E.J. Septimus, A. Srinivasan, T.H. Dellit, Y.T. Falck-Ytter, N.O. Fishman, C.W. Hamilton, T.C. Jenkins, P.A. Lipsett, P.N. Malani, L.S. May, G.J. Moran, M.M. Neuhauser, J.G. Newland, C.A. Ohl, M.H. Samore, S.K. Seo, K.K. Trivedi, Implementing an Antibiotic Stewardship Program: Guidelines by the Infectious Diseases Society of America and the Society for Healthcare Epidemiology of America, Clin Infect Dis 62(10) (2016) e51-77.

29. M. Devchand, C.M.J. Kirkpatrick, W. Stevenson, K. Garrett, D. Perera, S. Khumra, K. Urbancic, M.L. Grayson, J.A. Trubiano, Evaluation of a pharmacist-led penicillin allergy de-labelling ward round: a novel antimicrobia stewardship intervention, J Antimicrob Chemother 74(6) (2019) 1725-1730.

30. J. Molina, J.M. Cisneros, Editorial Commentary: A Chance to Change the Paradigm of Outcome Assessment of Antimicrobial Stewardship Programs, Clin Infect Dis 61(5) (2015) 807-8

31. E.C. Schuts, M. Hulscher, J.W. Mouton, C.M. Verduin, J. Stuart, H. Overdiek, P.D. van der Linden, S. Natsch, C. Hertogh, T.F.W. Wolfs, J.A. Schouten, B.J. Kullberg, J.M. Prins, Current evidence on hospital antimicrobial stewardship objectives: a systematic review and meta-analysis, The Lancet. Infectious diseases 16(7) (2016) 847-856.

32. D.M. Drekonja, G.A. Filice, N. Greer, A. Olson, R. MacDonald, I. Rutks, T.J. Wilt, Antimicrobial stewardship in outpatient settings: a systematic review, Infect Control Hosp Epidemiol 36(2) (2015) 142-52.

33. C. Pulcini, A. Huttner, CMI policy on antimicrobial stewardship research, Clin Microbiol Infect 24(2) (2018) 91-92.

34. C. Mathieu, B. Pastene, N. Cassir, I. Martin-Loeches, M. Leone, Efficacy and safety of antimicrobial deescalation as a clinical strategy, Expert Rev Anti Infect Ther 17(2) (2019) 79-88.

35. K. de With, F. Allerberger, S. Amann, P. Apfalter, H.R. Brodt, T. Eckmanns, M. Fellhauer, H.K. Geiss, O. Janata, R. Krause, S. Lemmen, E. Meyer, H. Mittermayer, U. Porsche, E. Presterl, S. Reuter, B. Sinha, R. Strauss, A. Wechsler-Fordos, C. Wenisch, W.V. Kern, Strategies to enhance rational use of antibiotics in hospital: a guideline by the German Society for Infectious Diseases, Infection 44(3) (2016) 395-439.

36. T.J. VanderWeele, M.B. Mathur, P. Ding, Correcting Misinterpretations of the E-Value, Ann Intern Med 170(2) (2019) 131-132.

37. T.J. VanderWeele, P. Ding, Sensitivity Analysis in Observational Research: Introducing the E-Value, Ann Intern Med 167(4) (2017) 268-274.

38. P. Ding, T.J. VanderWeele, Sensitivity Analysis Without Assumptions, Epidemiology (Cambridge, Mass.) 27(3) (2016) 368-77.

39. M.B. Mathur, P. Ding, C.A. Riddell, T.J. VanderWeele, Web Site and R Package for Computing E-values, Epidemiology (Cambridge, Mass.) 29(5) (2018) e45-e47. 
abo

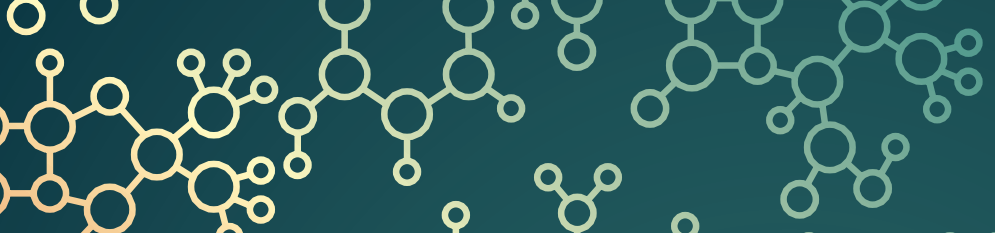
o

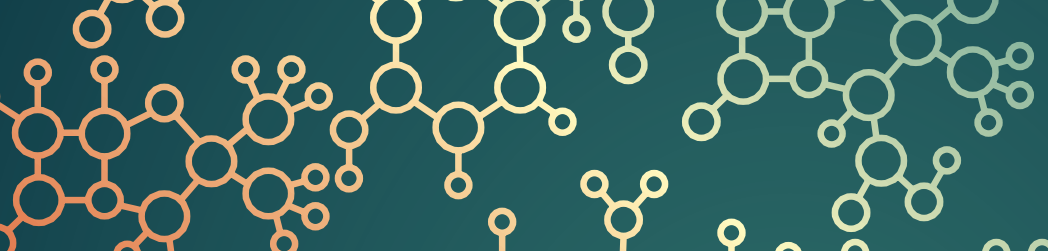

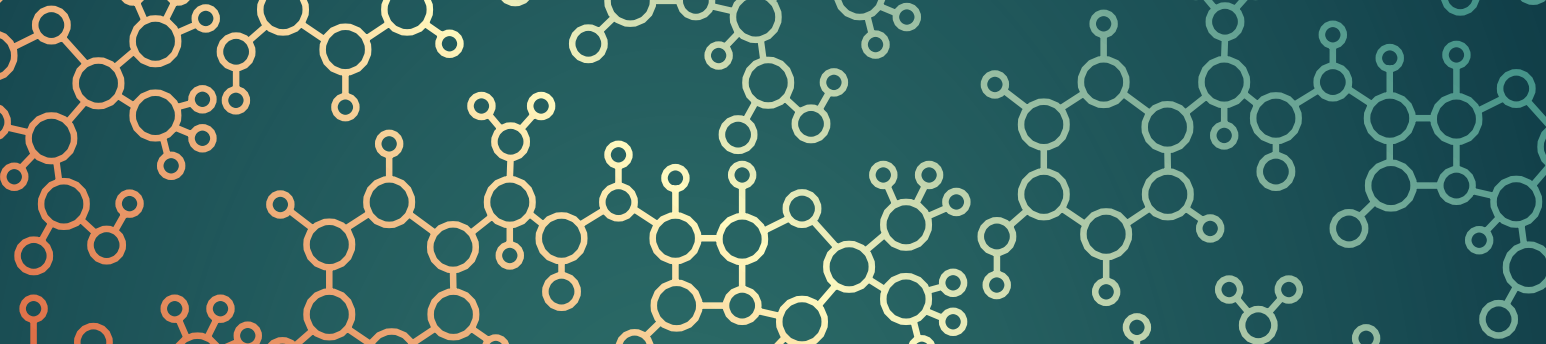

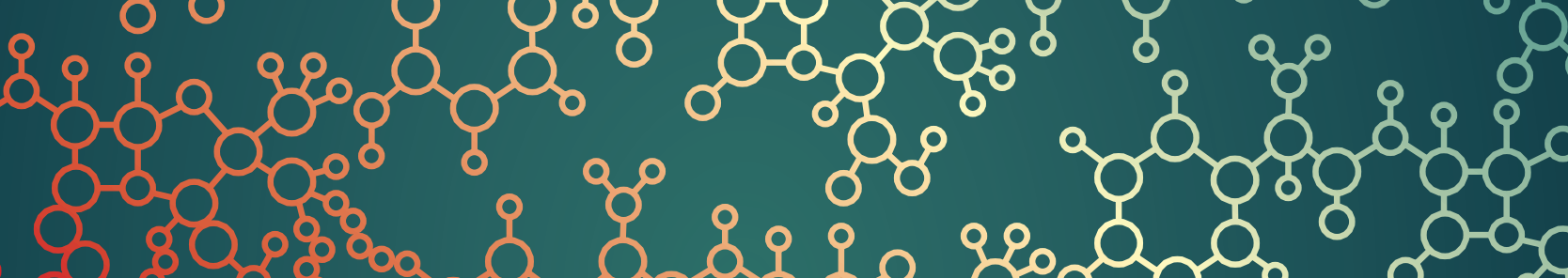
80\%

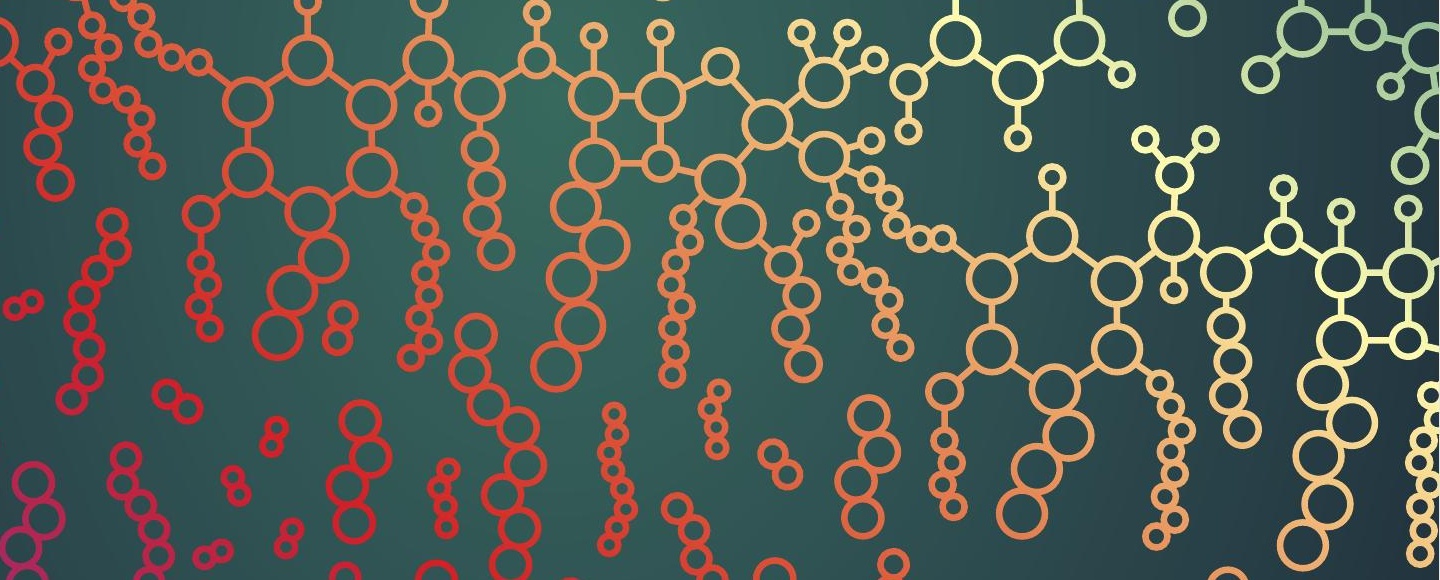
$88 \% 888$ $\circ 88$ \& 8

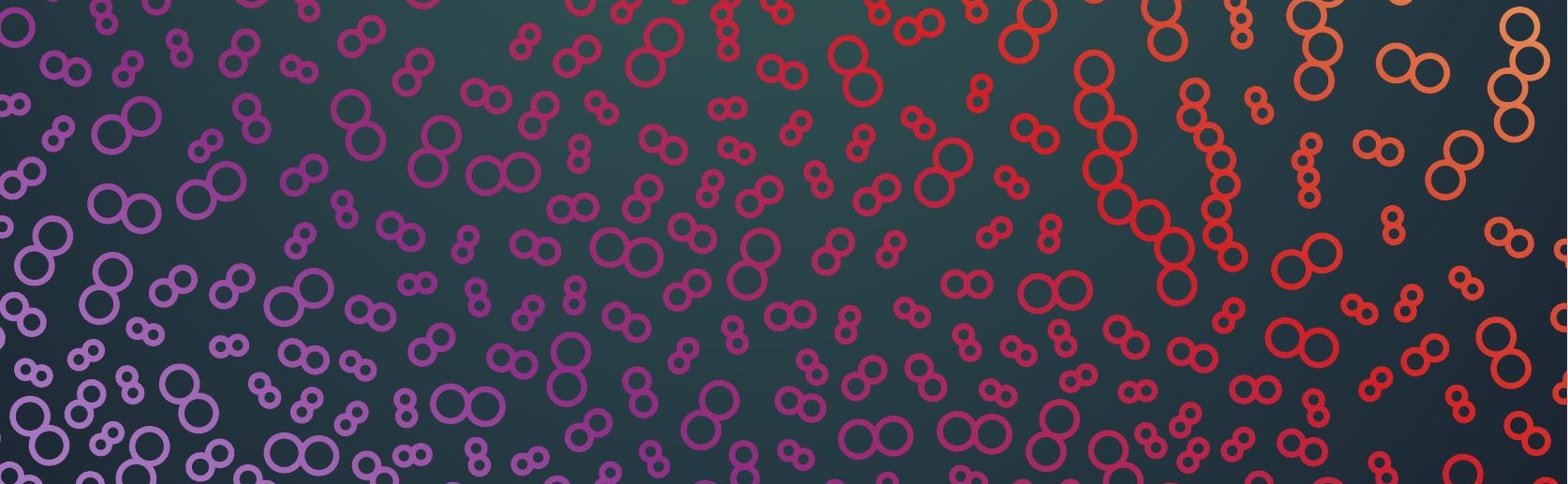


Appendices 


\section{DUTCH SUMMARY}

(samenvatting in het Nederlands) 
Dit proefschrift gaat over hoe de behandeling van patiënten met een thuis opgelopen pneumonie kan worden verbeterd met behulp van antibiotic stewardship interventies (Deel I) en hoe de methodologie van antibiotic stewardship studies kan worden verbeterd (Deel II). Deel I gaat in op het bewijs en de aanbevelingen voor juist gebruik van antibiotica bij ouderen en beschrijft de resultaten van een multicenter onderzoek met een antibiotic stewardship interventie bij patienten met een thuis opgelopen pneumonie, uitgevoerd in 12 Nederlandse ziekenhuizen. In Deel II wordt de methodologie van gepubliceerde antibiotic stewardship studies geëvalueerd en worden aanbevelingen gedaan voor het uitvoeren van toekomstige studies.

\section{Thuis opgelopen pneumonie}

Een pneumonie (longontsteking) is een veelvoorkomende acute aandoening van de lagere luchtwegen en veroorzaakt, met name bij ouderen, veel ziekte en kan leiden tot sterfte. Bij een pneumonie zijn de kleine vertakkingen van de longen en longblaasjes ontstoken, wat kan leiden tot klachten zoals koorts, hoesten en/of pijn bij inademing. Een pneumonie kan worden veroorzaakt door een bacterie of een virus, en minder vaak door schimmels of parasieten. Er wordt onderscheid gemaakt in een pneumonie opgelopen buiten het ziekenhuis (in het Engels: community-acquired pneumonia (CAP)) en een pneumonie opgelopen binnen het ziekenhuis (in het Engels: hospital-acquired pneumonia (HAP)). De belangrijkste reden voor dit onderscheid is een verschil in verwekker(s) en de daarmee samenhangende behandeling. In dit proefschrift wordt verder ingegaan op antibiotic stewardship in CAP.

De meest voorkomende verwekker van een CAP is de Streptococcus pneumoniae, ook wel pneumokok genoemd. De empirische antibiotische behandeling van een CAP is daarom meestal gericht op deze verwekker. Empirisch wil zeggen dat de verwekker nog niet bekendis als de behandeling wordt gestart. In afwachting van de microbiologische diagnostiek (kweken die worden ingezet om de verwekker te kunnen identificeren) kan er eventueel worden geswitcht naar een ander antibioticum. In de Nederlandse richtlijn voor de behandeling van CAP in volwassenen is de aanbevolen antibiotische behandeling gebaseerd op de ernst van de pneumonie. De ernst van de CAP wordt onderverdeeld in een milde, matig-ernstige en ernstige vorm. De ernst kan worden bepaald met drie methoden: PSI score (the Pneumonia Severity Index), de CURB-65 score of de pragmatische classificatie. De laatste classificatie is als volgt: Milde CAP indien behandeling thuis plaats vindt, matig-ernstige CAP indien opname op een verpleegafdeling gewenst is en ernstige CAP indien opname op een Intensive Care (IC) nodig is. De Nederlandse richtlijn heeft geen voorkeur voor één van deze methoden, maar beveelt wel aan consistent één score te gebruiken per ziekenhuis. Het advies voor een milde en matig-ernstige CAP is 
behandeling met penicilline of amoxicilline (smalspectrum antibiotica) omdat hiermee de pneumokok wordt gedekt. Mits er geen risicofactoren zijn voor andere verwekkers, zoals recent verblijf in het buitenland. Empirische antibiotische therapie wordt in de klinische praktijk vaak na 48 tot 72 uur geëvalueerd. Dit is het moment waarop het effect van de therapie kan worden beoordeeld en de patiënt klinisch stabiel is (of niet) én het moment dat de kweekresultaten bekend zijn. Patiënten met een ernstige CAP zijn klinisch zodanig ziek dat zij deze ruimte van evaluatie niet hebben en daarmee het risico op 48 tot 72 uur inadequate behandeling. Daarom worden deze patiënten vanaf het begin behandeld met breedspectrum antibiotica; bijvoorbeeld monotherapie met een fluorochinolon of een derde generatie cefalosporine i.c.m. ciprofloxacine.

In de dagelijkse praktijk blijkt dat de Nederlandse richtlijn voor de behandeling van een CAP bij volwassenen vaak niet wordt nageleefd, met name door het onnodig gebruik van te veel breedspectrum antibiotica in patiënten met een milde of matig-ernstige CAP. Voor het verhogen van de navolging van richtlijnen zoals deze, zijn Antibiotic Stewardship Programma's (ASP's) bewezen effectief gebleken.

\section{Antibiotic stewardship programma's (ASP's)}

Onjuist en/of onnodig gebruik van antibiotica draagt bij aan de ontwikkeling van antibioticaresistentie, stijging in zorgkosten en risico's op complicaties. Vóór 2015 werd in Nederland het juist gebruik van antibiotica vooral gestuurd door educatie, door de beschikbaarheid van nationale en lokale richtlijnen en door feedback en advies bij kweekresultaten door artsen-microbioloog. Maar in de dagelijkse praktijk bleken deze maatregelen niet voldoende. Met als gevolg dat per januari 2015 in alle Nederlandse ziekenhuizen een Antibiotic Stewardship team (A-team) verplicht werd gesteld vanuit de overheid. Dergelijke A-teams zijn verantwoordelijk voor het implementeren van ASP's. Het doel van ASP's is om onnodig en/of onjuist gebruik van antibiotica te minimaliseren, zonder nadelige gevolgen voor de individuele patiënt. Sindsdien zijn er meerdere (internationale) richtlijnen verschenen met aanbevelingen voor de ontwikkeling en implementatie van ASP's. In de Nederlandse richtlijn worden 15 ASP's sterk aanbevolen, voorbeelden hiervan zijn: (1) Schrijf empirische antibiotische therapie voor volgens de richtlijn en (2) verander empirisch antibiotisch beleid naar gerichte therapie zodra de resultaten van de kweken beschikbaar zijn (de-escalatie). Helaas is het bewijs dat ten grondslag ligt aan de meeste aanbevelingen van lage kwaliteit en vooral gebaseerd op observationeel onderzoek.

Een probleem bij observationeel onderzoek is de aanwezigheid van verstorende factoren, ook wel confounders genoemd. Om dit verder uit te kunnen leggen is het eerst belangrijk om te weten dat er in geneesmiddelonderzoek altijd een relatie wordt onderzocht tussen 
twee factoren: Blootstelling en uitkomst. Een voorbeeld van een onderzoeksvraag in zo'n onderzoek kan zijn: wat is het effect van geneesmiddel $X$ op het klinisch herstel van een patiënt? Geneesmiddel X kan dan worden vergeleken met patiënten die geneesmiddel X niet krijgen. Maar naast geneesmiddel $X$ zullen er veel meer factoren invloed hebben op het klinisch herstel van een patiënt, bijvoorbeeld leeftijd, geslacht of andere aanwezige ziekten. Bij het onderzoeken van het effect van geneesmiddel $\mathrm{X}$ is het dus ook belangrijk om te corrigeren voor de invloed van deze verstorende factoren. Behalve als in beide groepen alle verstorende factoren in dezelfde mate aanwezig zijn, dan zijn beide groepen vergelijkbaar en dan is correctie van confounders niet nodig om de juiste relatie tussen geneesmiddel $X$ en klinisch herstel te evalueren. In gerandomiseerd onderzoek zijn er vrijwel altijd vergelijkbare groepen, waarbij de blootstelling (hier: geneesmiddel $X$, of placebo) door toeval is toebedeeld aan de individuele patiënt. Daarom is confounding in deze onderzoeksopzet minder van belang. In observationeel onderzoek kan er wel een scheve verdeling zijn van één of meerdere confounders tussen de groepen, bijvoorbeeld een lagere leeftijd in de groep met geneesmiddel $X$ dan in de groep zonder geneesmiddel $X$. Leidt geneesmiddel $X$ dan tot een sneller klinisch herstel, of is het de lagere leeftijd? Daarom is het in observationeel onderzoek van essentieel belang om te corrigeren voor confounders. Een specifieke vorm van confounding is 'confounding by indication'. In dit geval is de indicatie van geneesmiddel $X$ op zichzelf de confounder. Als bijvoorbeeld bij ziekere patiënten vaker geneesmiddel $X$ wordt voorgeschreven dan bij niet zieke patiënten, dan zorgt de ernst van de ziekte (de indicatie) ervoor dat de twee groepen niet hetzelfde behandeld worden. Zonder correctie hiervoor kan er geen betrouwbare conclusie worden getrokken met betrekking tot de relatie tussen geneesmiddel en uitkomst.

Aangezien het bewijs onderliggend aan de aanbevelingen van ASP's vooral gebaseerd zijn op observationeel onderzoek van lage kwaliteit, zijn er methodologische adviezen nodig omtrent het goed uitvoeren van toekomstige observationele studies en goed uitgevoerde gerandomiseerde onderzoeken die ASP's evalueren.

\section{Deel I: Antibiotic stewardship interventies in CAP}

In Hoofdstuk 2 worden verschillende aspecten van antibioticagebruik bij oudere patiënten bij infecties van de lagere luchtwegen (en specifiek bij CAP) geëvalueerd die belangrijk zijn voor het bepalen van de juistheid van de voorschriften in deze populatie. Allereerst is er in de literatuur geen consensus over de definitie van 'juist gebruik van antibiotica'. Voorbeelden van verschillende definities die gebruikt zijn in studies met oudere patiënten zijn: (1) Juistheid op basis van gevoeligheid van de verwekker, (2) juistheid op basis van de diagnose, (3) juistheid op basis van de dosering, (4) juistheid op basis van de duur van 
de antibiotische therapie. Ondanks de verscheidenheid aan definities in de literatuur, is er wél consensus over de negatieve gevolgen van onjuist en/of onnodig gebruik van antibiotica. Wanneer er gekeken wordt naar de microbiologische etiologie (aantal en soort verwekkers) van de oudere patiënt is dit niet heel anders dan die van de jongere patiënt. Daarom is het advies om ook bij oudere patiënten te starten met beta-lactam monotherapie (bijvoorbeeld penicilline of amoxicilline). Tot slot hebben oudere patiënten meer kans op bijwerkingen van antibiotica, voornamelijk door het gebruik van veel geneesmiddelen naast elkaar (polyfarmacie) en door aanwezigheid van co-morbiditeiten (verschillende, vaak chronische, aandoeningen). Een goed opgezet ASP zou het juist voorschrijven van antibiotica kunnen verbeteren, maar studies specifiek uitgevoerd bij oudere patiënten zijn zeldzaam en dus hard nodig. Voorbeelden van mogelijke ASP's zijn: (1) Het verbeteren van de diagnostiek bij oudere patiënten (en daarmee de indicatie stelling en het daarmee wel of niet starten van antibiotica), en (2) ervoor zorgen dat de juiste dosering en duur van de antibiotische therapie wordt voorgeschreven.

Een voorbeeld van een goed opgezette antibiotic stewardship interventie wordt in Hoofdstuk 3 en 4 beschreven. Omdat de navolging van de CAP richtlijn in de praktijk laag is en ASP dit zou kunnen verbeteren, is de CAP-PACT ("Community-Acquired Pneumonia increasing Protocol adherence by Antibiotic stewardship in a stepped-wedge Clusterrandomized Trial) studie uitgevoerd in 12 Nederlandse ziekenhuizen. Deze antibiotic stewardship interventie bestond uit educatie (klinische lessen, e-learning, zakkaartjes en posters), motiveren van opinieleiders en audit en feedback. De interventie had als doel om artsen meer smalspectrum antibiotica te laten voorschrijven, en dus minder breedspectrum antibiotica. In totaal zijn er 4084 patiënten met een matig-ernstige CAP in de studie geïncludeerd (2235 in de controle- en 1849 in de interventie periode). De interventie bundel heeft een reductie van $27 \%$ in breedspectrum antibioticagebruik bewerkstelligd zonder negatieve gevolgen voor de patiënt.

In Hoofdstuk 4 wordt er dieper ingegaan op de details van de antibiotic stewardship interventie uit Hoofdstuk 3, door middel van een procesevaluatie van de afzonderlijke componenten. Van iedere component worden er vier aspecten beschreven: (1) De interventie zoals deze vooraf is bedacht, (2) de interventie zoals deze in de praktijk is uitgevoerd, (3) de daadwerkelijke blootstelling aan de interventie en (4) aanbevelingen om de afzonderlijke interventiecomponenten te verbeteren aan de hand van de ervaringen van de deelnemende artsen. De klinische lessen, het motiveren van opinieleiders en de audit en feedback werden beschouwd als de meest effectieve componenten van de interventie. De inhoud van de e-learning werd als goed beoordeeld, alleen door het fenomeen 'e-learning-vermoeidheid' was de daadwerkelijke respons lager dan verwacht. 
De poster werd door het merendeel van de artsen beoordeeld als onduidelijk en werd daardoor minder gewaardeerd als interventie. De aanbeveling is om frequent klinische lessen te geven, zakkaartjes uit te delen, opinieleiders aan te wijzen die blijvend actief betrokken zijn en dagelijks audit en feedback uit te voeren door het lokale A-team.

\section{Deel II: Methodologie van ASP studies}

In Hoofdstuk $\mathbf{5}$ worden de resultaten beschreven van een systematische review naar de studie-opzet van antibiotic stewardship studies. Verschillende aspecten van een studie-opzet zijn belangrijk om de validiteit van een studie te kunnen beoordelen en om de resultaten te kunnen vertalen naar de praktijk. Van de 12722 gevonden artikelen die antibiotic stewardship interventies evalueren, werden er 567 geïncludeerd. Het merendeel van de studies had een niet-gerandomiseerde studie-opzet, met als meest voorkomende het before-after design. In het algemeen was de kwaliteit van de studie-opzet van alle studies laag, en is die in de afgelopen jaren ook niet verbeterd. De meeste gepubliceerde artikelen rapporteerden geen klinische en/of microbiologische uitkomsten. Studies uitgevoerd in de eerste lijn en gesponsorde studies waren geassocieerd met een hogere kwaliteit. Deze tekortkomingen zouden moeten worden meegenomen in het opzetten van toekomstige studies die een antibiotic stewardship interventie evalueren. Daarmee wordt de kwaliteit van het bewijs, dat ten grondslag ligt aan de klinische praktijk, beter.

In Hoofdstuk 6 en 7 wordt er specifiek gekeken naar de methodologie van Antibiotic stewardship studies die correct gebruik van antibiotica door middel van de-escalatie van empirische antibiotische therapie evalueren. En dan met name van de nietgerandomiseerde Antibiotic stewardship studies. De-escalatie is het, waar mogelijk, versmallen van het spectrum van antibiotische therapie. Hoewel een dergelijk ASP wordt geadviseerd, is het bewijs voor de veiligheid hiervan gebaseerd op observationeel onderzoek. In een aantal van deze studies wordt zelfs een beschermend effect gezien van de-escalatie op de mortaliteit van patiënten, bij wie men de-escaleert. Hier is geen evidente biologische verklaring voor. Dit beschermende effect zal dus hoogstwaarschijnlijk worden veroorzaakt door confounding by indication. Er is sprake van confounding by indication als de indicatie voor de interventie (hier: de-escalatie van antibiotische therapie) ook een voorspellende factor is voor de uitkomst (mortaliteit). In de praktijk zal het spectrum van de antibiotische therapie alleen worden versmald als de patiënt klinisch stabiel is. Daarbij is klinische stabiliteit óók een sterk voorspellende factor voor mortaliteit (iemand die klinisch stabiel is zal een betere overleving hebben dan iemand die niet-klinisch stabiel is). Echter, klinische stabiliteit wordt zelden meegenomen als confounder in de bestaande literatuur. Daarom hebben we in Hoofdstuk 6 het potentiële effect van ongemeten confounding by indication door klinische stabiliteit gekwantificeerd in de relatie tussen de-escalatie en mortaliteit in patiënten met CAP. Dit hebben we 
gedaan door de klinische stabiliteit van patiënten met CAP tijdens ziekenhuisopname op dag 3 als dichotome confounder te simuleren. Deze gesimuleerde confounder was substantieel sterker dan alle andere bekende confounders in de associatie tussen deescalatie en mortaliteit. Dat betekent dat wanneer deze confounder niet mee wordt genomen in de analyse, dit zal leiden tot een sterke negatieve bias (en dus zal zorgen voor een vals-beschermend effect van de-escalatie). Goed uitgevoerde prospectieve studies zijn nodig om het daadwerkelijke effect van de-escalatie op mortaliteit te onderzoeken. Het is duidelijk dat de methodologie van ASP studies die het effect van de-escalatie op mortaliteit evalueren verbetering behoeven.

In Hoofdstuk 7 hebben we alle potentiële confounders in een directed acyclic graph (DAG) afgebeeld om alle associaties te laten zien die belangrijk zijn in de relatie tussen blootstelling (de-escalatie) en uitkomst (mortaliteit). Klinische stabiliteit is hierin een belangrijke tijdsafhankelijke confounder. Het advies is om de-escalatie als tijdsafhankelijke blootstelling in de analyse te includeren. En tot slot, om tevens inverse-probability-oftreatment weighted marginal structural models te gebruiken, om daarmee te corrigeren voor tijdsafhankelijke confounders.

In de general discussion wordt er dieper ingegaan op verschillende aspecten die belangrijk zijn in antibiotic stewardship in patiënten met een CAP. Zoals bijvoorbeeld de rol van ziekenhuisapothekers in ASP's. Waar ziekenhuisapothekers evident nog een belangrijke rol in kunnen spelen, onder andere vanwege de korte lijnen met de openbare apotheken, is het bewaken op de totale duur van antibiotische therapie en het de-labelen van onterechte penicilline allergieën. 
CONTRIBUTING AUTHORS 


\section{Julia Bielicki}

Paediatric Infectious Disease Research Group, St George's University of London, London, UK.

\section{Edwin Boel}

Department of Medical Microbiology, UMC Utrecht, Utrecht, The Netherlands.

\section{Marc M.J. Bonten}

Department of Medical Microbiology, UMC Utrecht, The Netherlands. Julius Center for Health Sciences and Primary care, UMC Utrecht, Utrecht, The Netherlands.

\section{Wendelien Dorigo-Zetsma}

Department of Medical Microbiology, Tergooi hospital, Hilversum/Blaricum, The Netherlands.

\section{Kelly D. Hendriks-Spoor}

Department of Medical Microbiology, Tergooi Hospital, Hilversum/Blaricum, The Netherlands. Julius Center for Health Sciences and Primary Care, UMC Utrecht, The Netherlands.

\section{Susanne M. Huijts}

Department of Pulmonary Medicine, Erasmus MC, Rotterdam, The Netherlands.

\section{Marlies E. Hulscher}

Radboud Center for Infectious Diseases, Radboud university medical center, Nijmegen, The Netherlands. Scientific Institute for Quality of Healthcare, Radboud university medical center, Nijmegen, The Netherlands.

\section{Jasmin Islam}

Department of Global Health and Infection, Brighton and Sussex Medical School, Falmer, UK.

\section{Martin J. Llewelyn}

Department of Global Health and Infection, Brighton and Sussex Medical School, Falmer, UK.

\section{Paul D. van der Linden}

Department of Clinical Pharmacy, Tergooi hospital, Hilversum/Blaricum, The Netherlands. 


\section{Jan Jelrik Oosterheert}

Department of Internal Medicine \& Infectious Diseases, UMC Utrecht, Utrecht, The Netherlands.

\section{Douwe F. Postma}

Department of Internal Medicine and Infectious Diseases, UMCG, Groningen, The Netherlands.

\section{Valentijn A. Schweitzer}

Department of Medical Microbiology, UMC Utrecht, The Netherlands.

\section{A. Sarah Walker}

MRC Clinical Trials Unit, University College London, London, UK.

\section{Cornelis H. van Werkhoven}

Julius Center for Health Sciences and Primary Care, UMC Utrecht, The Netherlands.

\section{Lufang Zhang}

Julius Center for Health Sciences and Primary Care, UMC Utrecht, The Netherlands. 


\section{ACKNOWLEDGEMENTS}

(Dankwoord) 
Veel mensen hebben mij direct of indirect geholpen bij de totstandkoming van dit proefschrift. Hiervoor wil ik graag een aantal personen in het bijzonder bedanken.

Beste prof. dr. Bonten, beste promotor, beste Marc, in 2015 mocht ik aansluiten bij jouw groep met promovendi. Voor mij was dit een prachtige kans om kennis te maken met verschillende specialismen en onderzoekers die zich bezighouden met de epidemiologie van infectieziekten. Dank voor je scherpzinnige en kritische blik en je motiverende woorden op de juiste momenten. Ik citeer: 'dit artikel is net als goede wijn, dat heeft ook een lange tijd nodig om te rijpen', na publicatie van een artikel waar ik ruim 4 jaar mee bezig was. Bedankt voor de prettige en leerzame samenwerking.

Beste dr. van der Linden, beste copromotor, beste Paul, allereerst wil ik je graag bedanken voor je aanstekelijke enthousiasme voor wetenschappelijk onderzoek en onderwijs op het gebied van Infectieziekten. De kansen die jij mij hebt gegeven om me verder te kunnen ontwikkelen op beide vlakken, waardeer ik enorm. Dank voor je hulp en je vertrouwen in een goede afloop. Ik kijk er naar uit weer met je samen te werken tijdens mijn uitwisseling naar Tergooi als AIOS Ziekenhuisfarmacie.

Beste dr. Dorigo-Zetsma, beste copromotor, beste Wendelien, het hebben van twee copromotoren vanuit verschillende vakgebieden heb ik enorm gewaardeerd. Jouw kennis en kunde op het gebied van de medische microbiologie, jouw ervaring in de kliniek en je praktische feedback heb ik erg gewaardeerd. Bedankt dat ik als apotheker onder jouw vleugels mij heb kunnen specialiseren in de infectieziekten.

Beste leden van de leescommissie: Prof. dr. Heijerman, Prof. dr. Hulscher, Prof. dr. De Jong, Prof. dr. Kluytmans en Prof. dr. Verheij. Hartelijk dank voor het lezen en beoordelen van dit proefschrift.

We hebben veel hulp gehad met het allergrootste project in dit proefschrift; De CAPPACT studie. Veel dank aan iedereen die hier aan heeft bijgedragen. In het bijzonder wil ik graag de volgende mensen nog bedanken.

Allereerst dank aan alle artsen (longartsen, internisten, artsen-microbioloog) en (ziekenhuis-)apothekers die het mogelijk hebben gemaakt dat de studie in negen ziekenhuizen in Nederland kon worden uitgevoerd: Amphia Ziekenhuis (Marco Grootenboers, Jan Kluytmans en Kees Verduin), Catharina Ziekenhuis (Heidi Ammerlaan en Ilse Overdevest), Diakonessenhuis (Akke van der Bij, Winnie de Bruin en Sanjay Sankatsing), LangeLand Ziekenhuis (Florence Ayuketah-Ekokobe, Johnny Daflaar en Michiel Haeseker), 
Máxima Medisch Centrum (Paul de Klaver en Dirk de Munck), Noordwest Ziekenhuisgroep (Wim Boersma en Wouter Rozemeijer), Tergooi ziekenhuis (Peter de Vries, Martin de Gier, Paul van der Linden en Wendelien Dorigo-Zetsma), Universitair Medisch Centrum Utrecht (Jan Jelrik Oosterheert), Ziekenhuisgroep Twente (Elske Engel-Dettmers, Bert Mulder en Milena Roorda-van der Vegt). Met ieder van jullie heb ik de samenwerking altijd als heel prettig ervaren, nogmaals veel dank voor jullie steun bij dit grote project.

Ook gaat een bijzondere dank uit naar diegene die 'de onderzoeks-motor' draaiende houden, want zonder jullie zijn wij als onderzoekers nergens: De research verpleegkundigen. Evelien Brands, José Groeneveld, Marjo van den Hurk Renée Ladestein en Linda van Mook. Ik had jullie vaker moeten overladen met bergen chocolade. Dank voor jullie hulp en de fijne samenwerking.

Tijdens de CAP-PACT studie hebben we ook ondersteuning gehad van twee studentassistenten. Thomas Boel, wij waren dringend op zoek naar een student die ons kon helpen, en jouw vader wist wel iemand. Jij hebt echt bergen aan werk verzet in een altijd goed gestemd humeur, hiervoor wil ik je ontzettend bedanken. Naomi Buntsma, ik heb zelden een student meegemaakt die zo hard en zo secuur werkt als jij. Ik prijs je werktempo en je proactieve houding. Wat baalde ik dat jij ons niet meer kon ondersteunen.

Tot slot hebben we in Tergooi nog ondersteuning gehad van mijn collega apothekersassistenten: Carla Beetz, Alice Nieuwenhuis, Annelies Scheepers, Edith de Vries en Wilma Zevering. Ontzettend bedankt voor jullie bijdrage.

Ook gaat mijn dank uit naar de volgende deelnemers van het CAP-overleg op vrijdagochtend: Edwin Boel, dank voor je altijd praktische benadering van mijn vraagstukken en specifiek voor je hulp bij het indelen van antibiotica op basis van hun spectrum. Jan Jelrik Oosterheert, dank voor je bijdrage aan het proefschrift en de mogelijkheid om een mini-stage te lopen bij de infectieziekten om de kliniek beter te leren kennen. Douwe Postma, naast dat Valentijn en ik jullie CAP-START studie een vervolg hebben kunnen geven, dank ik jou vooral voor je begeleiding tijdens mijn eerste publicatie. Wat hield jij de druk er goed op, want na een paar maanden hard werken was het artikel geaccepteerd met minimale revisie. Inez Bronsveld, dankjewel voor al je input en de prettige samenwerking. Lufang Zhang, jij bent de promovendus die ons werk voort gaat zetten met de CAP-NEXT studie. Ik zie jou als één van de masters in 'R', en dankzij jou kan ik mijzelf inmiddels uitroepen tot 'ggplot2-expert'. Henri van Werkhoven, last but definitely not least. Als assistant professor van Marc heb jij mij ook begeleid als een 'onofficiële derde' copromotor. Ik kan mij alleen maar aansluiten bij de motivering voor je 
prijs als supervisor van het jaar 2019. Bedankt voor je lage drempel voor epidemiologischstatistische vragen, je eindeloze geduld en je begeleiding zonder voorwaarden.

Ook de deelnemers van de extremely Early Wednesday Morning Meeting en de Wednesday Morning Meeting met hun altijd kritische houding hebben ervoor gezorgd dat mijn artikelen kwalitatief veel beter zijn geworden. Speciaal dank aan diegene die klaar stonden om de hoogte- en dieptepunten van mijn onderzoek aan te horen en die de jaarlijkse ECCMID-congressen onvergetelijk maakten: Annabel, Bastiaan, Darren, Diana, Douwe, Denise, Emma, Fien, Fleur, Gerrita, Henri, Janneke, Lufang, Kelly, Kirsten, Maaike, Meander, Nienke Pat, Nienke Pla, Sonja, Tess, Tessa, Thijs, Thomas, Tim, Valentijn en Wouter. Speciaal dank nog aan Janneke, voor je bijdrage aan een voor mij pas vrij laat ontdekt gebied: kwalitatief onderzoek. En Thomas, dank voor het maken van de jaarlijkse ECCMID-bingo.

Naast dat ik met name gestationeerd was in Tergooi, heb ik ook nog een tijdje een 'vaste' werkplek gehad in twee onderzoekskamers in het Julius centrum. Kamer 5.122: Birsen, Carmen, Clémence, Esther, Eveline, Feike, Loes, Lufang en Stephanie. Dank voor het plezier tijdens de 'kamer-uitjes', tijdens de Promovenski en op de kamer zelf. Ik vond het verfrissend om ook onderzoekers te leren kennen buiten de epidemiologieinfectieziekten groep. Evelien, dank dat je de tijd hebt genomen om mij een spoedcursus kwalitatief onderzoek te geven. Van Geuns kamer 5.07: Denise, Fien en Tess. Dank voor jullie steun in de laatste maanden, de meest stressvolle periode van het promotietraject. Denise, wat vond ik het fijn dat jij dezelfde deadlines had, hierdoor voelde het afronden van mijn proefschrift meer als gedeelde smart. Dank dat je mij uitnodigde om bij jullie op de kamer te komen werken.

Buiten alle steun en hulp van bovenstaande personen draag ik ook mijn collega's van het Tergooi een warm hart toe. Allereerst mijn collega (ziekenhuis)-apothekers: Alper, Anke, Gijsje, Ineth, Jeffrey, Jill, Jolande, Koen, Kris, Linda, Madelon, Paul, Rashudy, Suzan, Willemien. Een speciale dank aan Jeffrey, dankzij jou zagen de pijlen in mijn de-escalatie grafiek er bijzonder mooi uit. Jill, jij bent vanaf het begin van mijn tijd in Tergooi heel waardevol voor mij geweest. We hebben samen gelachen, maar ook samen gehuild (van het lachen). Wat een eer was het toen je mij vroeg om jullie ceremoniemeester te zijn tijdens de bruiloft met je geliefde: Arief. Arief, ook jou wil ik enorm bedanken voor je methodologische hulp tijdens een van mijn eerste artikelen. Je onuitputtelijke geduld en de manier waarop jij ingewikkelde methodologie kan uitleggen is bewonderingswaardig. Koen en Linda, mede apotheker-onderzoekers; succes met de laatste loodjes van jullie proefschriften. Koen, het laatste jaar dat wij naast elkaar zaten was misschien niet altijd 
bevorderlijk voor de voortgang van dit proefschrift, maar wel voor de lachspieren. Tevens kon ik mijn 'R' expertise met je delen. Linda, als mijn trouwe reisgenoot namen wij altijd de dag nog even samen door tijdens onze autoritjes. Kris, wat hebben wij met $z$ 'n twee zitten stressen, maar gelukkig ook veel gelachen, als eerste twee promovendi van de Tergooi ziekenhuisapotheek. Rashudy, wat kan ik genieten van onze gedeelde passie voor rake quotes op Instagram. En, niet geheel onbelangrijk, wil ik je bedanken dat je mij op een blinddate hebt gezet met Philippe. Marjon en Aurelia, ik kijk er naar uit om jullie weer te zien als collega-AIOS in het St. Antonius ziekenhuis. Tot slot, Jolande, als een soort moeder de gans ontferm jij je over de $A(N) I O S$, wat ik altijd als heel prettig heb ervaren. Mede door jou voelde Tergooi als een warm bad. Dank voor al je wijsheid, het meedenken over werk-gerelateerde toekomstplannen en je zeer gewaardeerde kort-door-de bocht eerlijkheid.

Elsje jij geeft iedere nieuwe medewerker van de afdeling Klinische Farmacie een warm welkom. Dank voor je gezelligheid tijdens onze borrels, je schouder als ik hem even nodig had en je humor. Ook Yke en Jacqueline, 'collega's achter de muur', wil ik bedanken voor de oprechte interesse en het aanhoren van mijn hoogte- en dieptepunten bij het tot stand komen van dit proefschrift.

Tot slot wil ik graag de volgende leden van het Antibiotica-team van Tergooi bedanken voor alle leerzame momenten: Leendert Bakker, Jarne van Hattem, Matthijs Silbermann, Cornelis Timmerman en Peter de Vries.

En dan zijn mijn laatste werk gerelateerde dankwoorden gericht aan mijn paranimfen: Valentijn Schweitzer en Kelly Hendriks. Valentijn, mede CAP-PACTer, wat ben ik blij dat wij deze grote studie samen hebben mogen uitvoeren. Met zijn tweeën is het uitvoeren van een dergelijke studie met name heel praktisch, maar vooral ook veel leuker. Wat hebben wij veel ritjes samen in de trein en in de auto gemaakt voor die 54 klinische lessen in 9+ verschillende ziekenhuizen door heel Nederland. Urenlang hebben we elk detail van Game Of Thrones en The Walking Dead besproken. Met als kanttekening dat jij mij altijd nog extra informatie kon verschaffen die je had gelezen in blogs of op andere internetpagina's. En dat is ook wat jou typeert. Jij gaat de diepte in tot je het naadje van de kous hebt gevonden. Jouw epidemiologische kennis, je gouden geduld, je ideeën voor nieuwe studies, maar ook je humor maakte je een hele fijne collega. Lieve Kelly, ik ken weinig personen die altijd eerst aan een ander denken en dan pas aan zichzelf. Net in dienst, mee naar je eerste ECCMID congres en iemand was haar tandenborstel vergeten. Jij kwam snel daarna ongevraagd met een nieuw gekochte tandenborstel, zonder dat iemand je iets had gevraagd. Dit typeert jou, jij bent een stille kracht. Twee jaar na mij 
ben je gestart als mijn partner-in-crime in antibiotic stewardship onderzoek in Tergooi, en wat was ik blij dat je er bij bent gekomen. Ondanks dat je een zwaarder traject achter de rug hebt dan menig ander onderzoeker, kan ik je alleen maar prijzen om je enorme doorzettingsvermogen. Ontzettend bedankt dat je ook in deze periode voor mij klaar stond. En heel fijn dat jullie mij beide bijstaan tijdens de verdediging van mijn proefschrift.

Lieve Marthe, ontzettend bedankt voor het prachtige ontwerp voor de cover van dit proefschrift! Je hebt een gave.

Lieve jaarclub Kampai, dank voor jullie onvoorwaardelijke steun, gezellige borrels, vakanties, en alles wat wij hebben meegemaakt in de ruim dertien jaar dat wij elkaar kennen. Ik vind het fijn dat wij elkaar na zo'n lange tijd nog steeds vaak zien. En sorry voor alle borrels, feesten en etentjes die ik op het laatste moment toch moest afzeggen vanwege deadlines. Lieve Cor en Reen, lieve Lucie, de Lustrumreis is er nooit van gekomen, maar ik heb er wel een hechte vriendschap aan over gehouden. En ik kijk er naar uit om samen met jullie onze roadtrip te maken door Canada. Lieve Cath, Lo en Eef, 'De Utrechtse vriendinnen', deze titel kunnen we maar beter wijzigen in 'Half om Half', en ondanks dat ik nu in Amsterdam woon kijk ik er naar uit om onze borrels voort te zetten. Lieve Sjaan, ook al woon je helemaal in het verre Oosten, wij pakken met gemak de draad weer op als we elkaar weer zien. Lieve Marie, dank voor al je promotietips vanuit het verre Canada en ik zie je snel. Lieve Rob, Lies en Els ook jullie altijd oprechte interesse vond ik erg fijn.

Lieve vriendinnetjes van Farmacie: Frederique en Jacqueline. Fred, samen met jou fietsen of een lesje Body Balance volgen was voor mij een erg fijn naast de drukte van de promotie. En dank voor de momenten ná het sporten, waar in ik nog mijn ups- en downs kon evalueren. Jacq, tijdens mijn studie stond je al voor mij klaar en nu is dat nog steeds zo. We zien elkaar de laatste jaren wat minder, maar ik vind het fijn dat we zonder problemen de draad gewoon weer oppakken als we elkaar weer zien.

Lieve Floor, van al mijn vriendinnen ken ik jou het langst, al zo'n 29 jaar. Ik heb altijd een beetje jouw voetsporen gevolgd richting de gezondheidszorg, het promoveren en nu ook het wonen in Amsterdam. Dank dat je altijd klaar stond om mijn onderzoeks-perikelen aan te horen en je steun en adviezen om weer met goede moed verder te kunnen gaan.

Lieve familie, dank dat jullie altijd voor mij klaar staan. Lieve mama, niemand is denk ik zo trots op mij als dat jij dat bent. Fijn dat de deur in Arnhem altijd openstaat om even 'bij te tanken'. Lieve Astrid, mijn tweelingzus, je woont nu al een paar jaar in London maar 
toch voelt het alsof je heel dichtbij bent, omdat we elkaar vrijwel dagelijks spreken. I will visit you and Arnold soon in London. Also to give a hug to my baby cousin. Lieve Mark en Jacqueline, bij jullie op de Brabantse boerderij is het altijd een beetje thuiskomen, dank voor jullie warme nest waar ik altijd graag naar toe ga. Lieve Sven, wat fijn dat je toch nog een beetje in mijn buurt woont nu ik naar Amsterdam ben vertrokken. Et Julien, vous complétez notre famille. Lieve papa en Bo, ook Maastricht behoort tot een van mijn toevluchtsoorden voor de nodige ontspanning. Ik vind het heerlijk om bij jullie even een weekendje bourgondisch te leven, en met de racefiets een rondje België te doen. Lieve Janet en René, dank voor jullie telefoontjes, kaartjes en etentjes, die hebben mij veel steun geboden tijdens mijn promotie. En tot slot: Arie, Joke en Elwin met jullie erbij voelt Kerst altijd weer compleet.

Lieve Philippe, ruim twee jaar geleden heeft Rashudy, met toestemming, onze nummers uitgewisseld. Wat ben ik blij dat we beide de moed hadden om 'blind' af te spreken. Ik vind het fijn dat ik bij jou volledig mijzelf kan zijn, inclusief mijn meest stressvolle versie. En zelfs dan lukt het jou om mij weer met beide benen op de grond te zetten. Bedankt dat je altijd voor mij klaar staat en voor je betrokkenheid, steun en motivatie bij dit proefschrift. Ik kijk uit naar alle momenten die wij nog gaan beleven samen.

Inger van Heijl, maart 2020. 
CURRICULUM VITAE 
Inger van Heijl was born on August 22nd 1986 in Velp and grew up in Arnhem, the Netherlands. After completing secondary school at the 'Lorentz College' in Arnhem, she studied Pharmacy at the University of Utrecht. As part of the master's programme she completed her research project at the department of Clinical Pharmacy of Tergooi hospital in Blaricum and Hilversum in collaboration with the department of Neurology and Neurosurgery of the University Medical Center (UMC) of Utrecht. This epidemiological study resulted in a publication on the treatment of epilepsy in daily practice. After this period, she aspired to further her academic skills.

After obtaining her master's degree in 2013 she started working as a pharmacist at the Clinical Pharmacy department of Tergooi hospital in Blaricum and Hilversum. In September 2014 she continued her work as a pharmacist and combined this with antibiotic stewardship team tasks. At his time she also started a PhD trajectory in the field of Infectious Diseases at the Julius Center for Health Science and Primary care under the supervision of Prof. dr. M.J.M. Bonten (UMCU, Julius Center), dr. P.D. van der Linden (Tergooi hospital) and dr. J.W. Dorigo-Zetsma (Tergooi hospital). During her PhD trajectory she completed a postgraduate Epidemiology master with a specialization in Pharmacoepidemiology. From April 2020 onwards, she will start her training in hospital pharmacy at the St. Antonius Hospital in Nieuwegein and Utrecht under the supervision of dr. E.M.W. van de Garde and Prof. dr. C.A.J. Knibbe. 


\section{LIST OF PUBLICATIONS}




\section{Publications related to this thesis}

van Heijl I, Schweitzer VA, van der Linden P, Bonten MJM, van Werkhoven CH. Impact of antimicrobial de-escalation on mortality: a literature review of study methodology and recommendations for observational studies. Expert Review of Anti-infective Therapy, DOI: $10.1080 / 14787210.2020 .1743683$

van Heijl I, Schweitzer VA, Boel CHE, Oosterheert JJ, Huijts SM, Dorigo-Zetsma JW, van der LindenPD, Bonten MJM, van Werkhoven $\mathrm{CH}$. Confounding by indication of the safety of de-escalation in community-acquired pneumonia: A simulation study embedded in a prospective cohort. PLoS One. 2019;14(9):e0218062. Published 2019 Sep 27. doi:10.1371/ journal.pone.0218062.

Schweitzer VA, van Heijl I, van Werkhoven CH, Islam J, Hendriks-Spoor KD, Bielicki J, Bonten MJM, Walker AS, Llewelyn MJ, Consensus on Antimicrobial Stewardship Evaluations (CASE) study group. The quality of studies evaluating antimicrobial stewardship interventions: a systematic review. Clin Microbiol Infect. 2019;25(5):555-561. doi:10.1016/j.cmi.2018.11.002.

van Heijl I, Schweitzer VA, Zhang L, van der Linden PD, van Werkhoven CH, Postma DF. Inappropriate Use of Antimicrobials for Lower Respiratory Tract Infections in Elderly Patients: Patient- and Community-Related Implications and Possible Interventions. Drugs Aging. 2018;35(5):389-398.

\section{Publications not related to this thesis}

Schweitzer VA, van Werkhoven CH, van Heijl I, R.F. Smits, C.H.E. Boel, M.J.M. Bonten, D.F. Postma, J.J. Oosterheert. Relevance of healthcare-associated pneumonia for empirical antibiotic therapy in the Netherlands. Neth J Med. 2018;76(9):389-396.

Berrevoets MA, Ten Oever J, Sprong T, van Hest RA, Groothuis I, van Heijl I, Schouten JA, Hulscher ME, Kullberg B. Monitoring, documenting and reporting the quality of antibiotic use in the Netherlands: a pilot study to establish a national antimicrobial stewardship registry. BMC Infect Dis. 2017:17(1):565. Published 2017 Aug 15. doi:10.1186/s12879-0172673-5.

Wassenaar M, van Heijl I, Leijten FS, van der Linden P, UijI SG, Egberts ACG, Carpay JA. Treatment of epilepsy in daily clinical practice: have outcomes improved over the past 10 years?. J Neurol. 2013;260(11):2736-2743. doi:10.1007/s00415-013-7058-0 


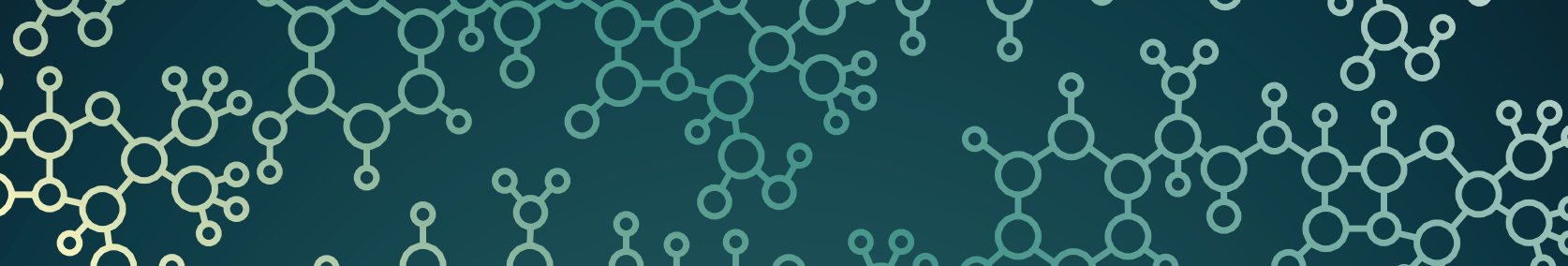

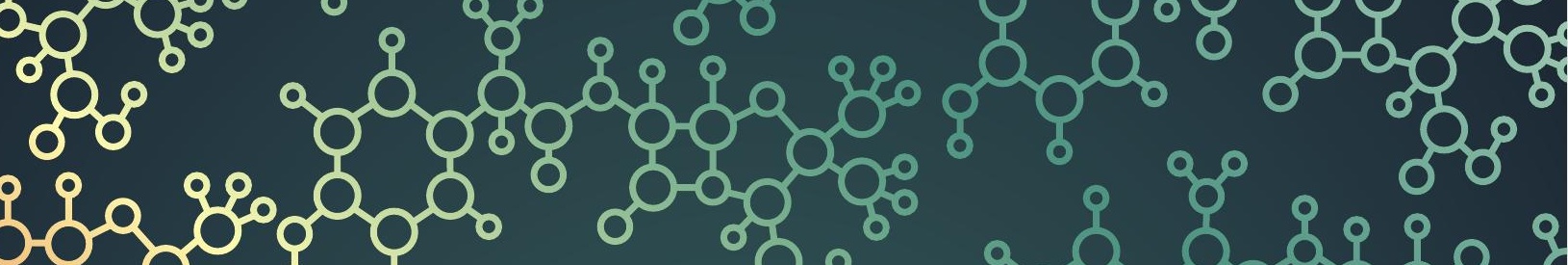

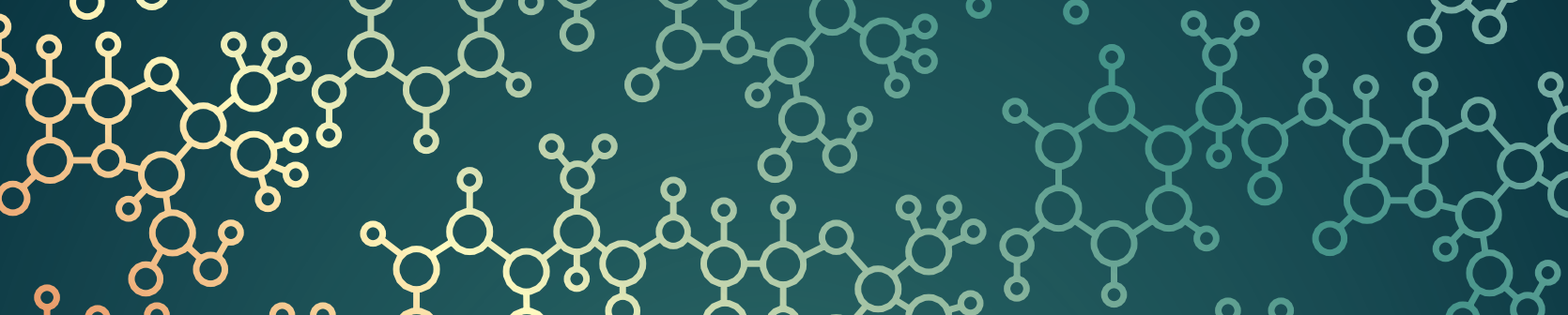

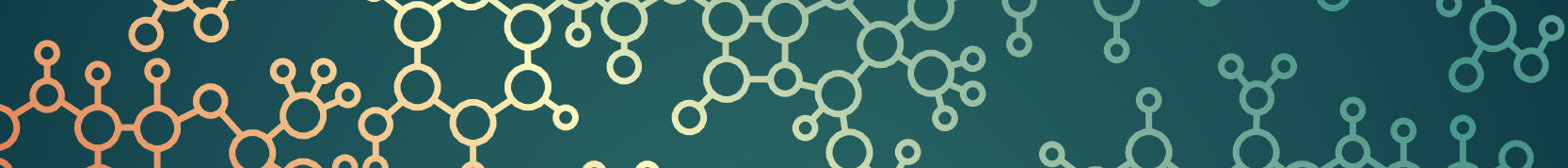

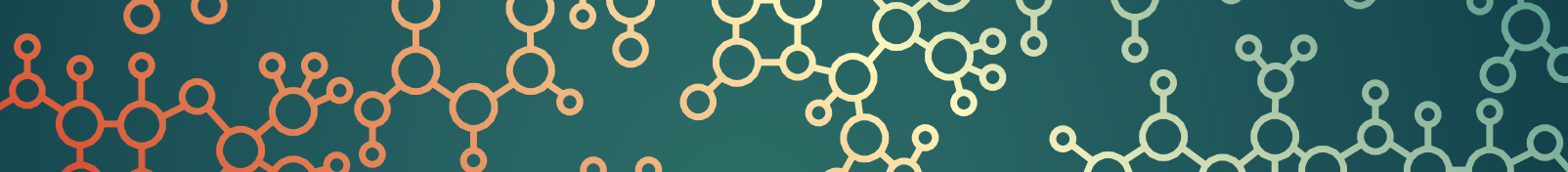
0.

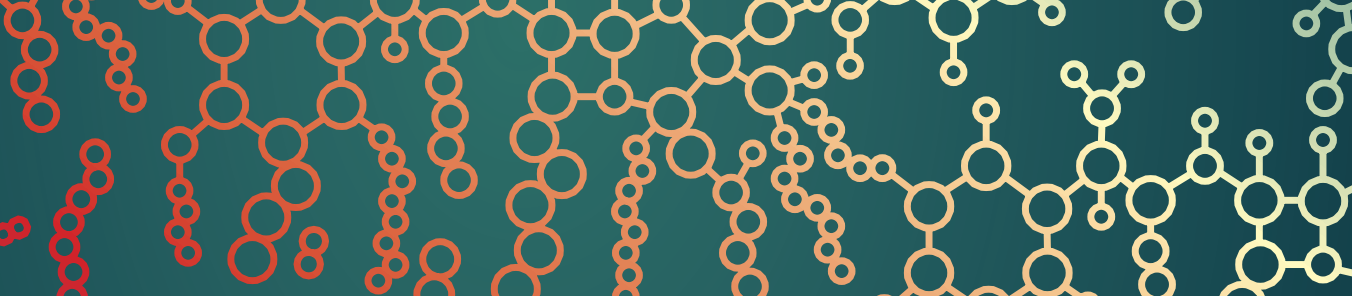
8 \& 8 \& 00 \% 8 $88 \% 8 \% 8$

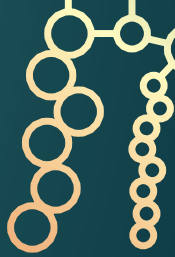

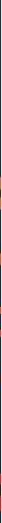

